\title{
Prediction of Tip Vortex Cavitation for Ship Propellers
}




\section{Prediction of Tip Vortex Cavitation for Ship Propellers}

A. I. Oprea 
Prediction of Tip Vortex Cavitation for Ship Propellers A.I. Oprea

Thesis University of Twente, Enschede, The Netherlands With ref. - With summary in Dutch.

ISBN 978-90-365-3579-3

Cover design: Proefschriftmaken.nl || Uitgeverij BOXPress Printed by: Proefschriftmaken.nl || Uitgeverij BOXPress Published by: Uitgeverij BOXPress, 's-Hertogenbosch

Copyright (c) 2013 by A.I. Oprea 


\title{
PREDICTION OF TIP VORTEX CAVITATION FOR SHIP PROPELLERS
}

\author{
PROEFSCHRIFT
}

ter verkrijging van

de graad van doctor aan de Universiteit Twente,

op gezag van de rector magnificus,

prof. dr. H. Brinksma,

volgens besluit van het College voor Promoties

in het openbaar te verdedigen

op vrijdag 20 december 2013 om 10.45 uur

door

Ana Iulia Oprea

geboren op 27 januari 1979

te Bucuresti, Romania 
Dit proefschrift is goedgekeurd door de promotoren:

prof. dr. ir. H.W.M. Hoeijmakers

prof. dr. ir. T.J.C. van Terwisga 


\section{Summary}

An open propeller is the conventional device providing thrust for ships. Due to its working principles, regions with low pressure are formed on its blades specifically at the leading edge and in the tip region. If this pressure is becoming lower than the vapor pressure, the cavitation phenomenon is initiated, i.e. in these low pressure regions liquid turns into vapor. Thus, cavitation results in regions filled with vapor, present on and close to the propeller blades. This causes nuisance effects like noise, vibrations, erosion and even thrust break-down in its most severe cases. Due to these unwanted features of cavitation, the design of propeller blades has to account for cavitation. This requires a computational tool to predict propeller cavitation, already in the design stage of the propeller. Such a computational tool should be suited for prediction of the cavitating flow around model-scale and full-scale propellers.

Model-scale tests, as a form of studying cavitation phenomena, have been used from the beginning of recorded history on cavitation. This procedure is well established and fine-tuned with well-recognised capabilities. However, the main drawbacks of the method based on model-scale tests are: the scaling of the tip vortex cavitation inception and the scaling of the performance of the cavitating propellers. Procedures and recipes for an accurate scaling have been developed, however, their use is often failing for the modern geometrically more complex propellers. Therefore, in the last decades there has been an increasing effort in using numerical simulation methods to predict cavitation on propellers. These methods range from potential-flow methods to Large-Eddy Simulation methods.

In the present study, the focus is on using a high-fidelity mathematical model of the flow, implemented in a relatively fast numerical method, such that the method can be used within industry. The approach based on the ReynoldsAveraged Navier-Stokes (RANS) equations has been selected. This method is used in achieving the goal of the thesis: the prediction of the inception of tip vortex cavitation and the prediction of the development of the tip vortex. For this purpose, a number of benchmark test cases have been used to vali- 
date the chosen approach and to investigate the basic flow characteristics of cavitation. Subsequently, the method has been applied to a number of open propellers, both at model and at full scale. 


\section{Samenvatting}

Een open scheepsschroef is de meest gebruikte methode om stuwkracht te leveren voor de voorstuwing van een schip. Door zijn werkingsprincipe worden gebieden met lage druk gevormd op de bladen, in het bijzonder op de intreezijde en nabij de tip. Wanneer deze druk lager wordt dan de dampdruk, begint het cavitatiefenomeen, met andere woorden in deze gebieden met lage druk verandert water in waterdamp. Door het effect van cavitatie onstaan er gebieden met waterdamp op en dichtbij het blad. Dit zorgt voor hinderlijke effecten zoals geluid, trillingen, erosie en zelfs in de ergste gevallen voor stuwkracht-afname. Door deze ongewensde eigenschappen van cavitatie moet al tijdens het ontwerp van propellerbladen rekening worden gehouden met cavitatie. Hiervoor is een reken methode model nodig om al in het ontwerpproces cavitatie op de schroef te kunnen voorspellen. Deze reken methode model moet geschikt zijn om het cavitatie gedrag te voorspellen voor zowel schroeven op modelschaal als op ware-grootte.

Vanaf de tijd dat cavitatie beschreven is zijn modelschaaltesten gebruikt om het cavitatiefenomeen te bestuderen. De procedure is uitgebreid vastgelegd en is een in de industrie erkende methode. De voornaamste moeilijkheden van het gebruik van modeltesten om cavitatie te bestuderen zijn: het opschalen van de inceptie van tipwervel-cavitatie en het opschalen van het rendement van de caviterende schroef. Procedures en voorschriften zijn ontwikkeld voor een nauwkeurige schaling, maar hun toepassing is ontoereikend voor moderne, geometrisch meer complexe schroefontwerpen. Zodoende zijn er in de laatste decennia steeds meer onderzoek verricht om numerieke simulatiemethodes te gebruiken om cavitatie op schroeven te voorspellen. Deze methodes variëren van potentiaalstroming methodes tot methodes voor Large-Eddy Simulaties.

In de huidige studie ligt de focus op het gebruik van een high-fidelity wiskunding stromingsmodel geimplementeerd in relatief snelle numerieke methodes, zodat de methode gebruikt kan worden in de industrie. Er is gekozen voor een aanpak gebaseerd op de Reynolds-Gemiddelde Navier-Stokes (RANS)vergelijkingen. Deze methode is gebruikt om het doel van dit proefschrift te 
bereiken, namelijk: het voorspellen van de inceptie van tipwervelcavitatie en het voorspellen van de ontwikkeling van de tipwervel. Met dit doel zijn een aantal benchmarks gekozen om de gekozen aanpak the valideren en om de karakteristieken van caviterende stromingen te onderzoeken. Vervolgens is de methode toegepast op een aantal open schroeven, voor zowel modelschaal als ware-grootte schaal. 


\section{Contents}

Summary

Samenvatting iii

Contents $\quad$ v

1 Introduction $\quad 1$

1.1 Propeller . . . . . . . . . . . . . . . . . . . . . . . 1

1.2 Cavitation . . . . . . . . . . . . . . . . . 3

1.2.1 History . . . . . . . . . . . . . . . . . 3

1.2 .2 Definition . . . . . . . . . . . . . . 4

1.2.3 Stressing of liquids . . . . . . . . . . . . . . 5

1.2.4 Cavitation number . . . . . . . . . . . 6

1.3 Propeller cavitation . . . . . . . . . . . . . . . . 7

1.3.1 Cavitation types . . . . . . . . . . . . 7

1.3.2 Tip vortex cavitation . . . . . . . . . . . . . . 9

1.3.3 Cavitation nuisance . . . . . . . . . . . . . 10

1.4 Motivation and Objectives . . . . . . . . . . . . . . 11

1.5 Approach . . . . . . . . . . . . . . . . . . . . . 12

1.6 Outline thesis . . . . . . . . . . . . . . . 15

2 Mathematical modeling $\quad 17$

2.1 Conservation Principles . . . . . . . . . . . . . . . . . . . 17

2.1.1 Introduction . . . . . . . . . . . . . . . . . 17

2.1.2 General Principle of Conservation . . . . . . . . . . . 18

2.1.3 Mass Conservation . . . . . . . . . . . . . . 20

2.1.4 Momentum Conservation Principle . . . . . . . . . 21

2.1.5 Energy Conservation Principle . . . . . . . . . . . . 22

2.2 Mathematical models . . . . . . . . . . . . . . . . . 23

2.2.1 Navier-Stokes equations . . . . . . . . . . . . 23

2.2.2 Reynolds Averaged Navier-Stokes model . . . . . . . . 26 
2.2.3 Turbulence models . . . . . . . . . . . . . . . 28

2.2.4 Wall functions . . . . . . . . . . . . . . . . . . . . . . . . . . . . . . 32

2.3 Cavitation Modeling . . . . . . . . . . . . . . . . 34

2.3.1 Introduction . . . . . . . . . . . . . . . . . . . . . . . . . . . .

2.3.2 Governing Equations . . . . . . . . . . . . . . . 36

2.3.3 Rayleigh-Plesset Cavity Source Terms . . . . . . . . . . 37

2.3.4 Volume of Fluid method . . . . . . . . . . . . . . . 38

2.3.5 Mass transfer equation . . . . . . . . . . . . . 39

3 Vortex detection and visualization $\quad 41$

3.1 Vortex definition . . . . . . . . . . . . . . . . . . . . . . . . . . . 41

3.2 Vortex detection methods . . . . . . . . . . . . . . . . 42

3.2 .1 Intuitive Methods . . . . . . . . . . . . . . . . . 42

3.2.2 Velocity Gradient Tensor Methods / Galilean Invariant

Methods . . . . . . . . . . . . . . . 44

3.2 .3 Discussion . . . . . . . . . . . . . . . 52

3.3 Vortex visualization methods . . . . . . . . . . . . . 53

3.4 Discussion on the selected method . . . . . . . . . . . . . 53

3.4.1 Detection . . . . . . . . . . . . . 54

3.4 .2 Grid optimization . . . . . . . . . . . . . . . 54

3.4.3 Visualization ................... 57

3.5 Conclusion . . . . . . . . . . . . . . . . . 57

4 Assessment of uncertainty $\quad 59$

4.1 Introduction . . . . . . . . . . . . . . . . . 59

4.2 Verification methodology . . . . . . . . . . . . . 60

4.3 Verification CFD method: steady flow . . . . . . . . . . . . 61

4.3.1 Geometry, Conditions, and Benchmark Data . . . . . . 61

4.3.2 Computational Grids . . . . . . . . . . . . 63

4.3.3 Verification of Integral Variables: Elliptic 11 Rake Hydrofoil . . . . . . . . . . . . . . . . 65

4.3.4 Verification of local variable: minimum pressure coefficient at mid-span . . . . . . . . . . . . . . 70

4.3.5 Conclusions Steady Flow: Grid Guidelines . . . . . . . 71

4.4 Verification CFD method: unsteady flow . . . . . . . . . . 73

4.4.1 Geometry: 2D NACA 0015 section . . . . . . . . . . 74

4.4.2 Computational domain and mesh . . . . . . . . . . 76

4.4.3 Settings of numerical parameters . . . . . . . . . . 77

4.4.4 Results wetted flow . . . . . . . . . . . . . 78

4.4.5 Results cavitating flow, $\sigma=1.0 \ldots \ldots$. . . . . 83 
4.4.6 Unsteady Flow Conclusions: Turbulence and Time step Guidelines . . . . . . . . . . . . . . . . 94

4.5 Conclusions . . . . . . . . . . . . . . . . . . . . . . 95

5 Underlying Flow Characteristics $\quad 97$

5.1 Introduction . . . . . . . . . . . . . . . . . . . . . 97

5.2 Delft Twist-11 hydrofoil . . . . . . . . . . . . . . . . . 98

5.2 .1 Geometry . . . . . . . . . . . . . . . . . . . 98

5.2.2 Computational domain and mesh . . . . . . . . 100

5.2 .3 Numerical parameters . . . . . . . . . . . . . . 101

5.2.4 Results for wetted flow . . . . . . . . . . . . . . . 102

5.2.5 Results for cavitating flow, $\sigma=1.0 \ldots \ldots$. . . . 105

5.2 .6 Conclusions . . . . . . . . . . . . . . . . . . . . . . 112

5.3 3D Elliptic 11 rake hydrofoil . . . . . . . . . . . . . . 113

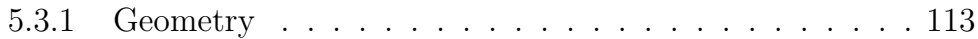

5.3.2 Computational domain and mesh . . . . . . . . . . . 114

5.3.3 Numerical parameters . . . . . . . . . . . . . 115

5.3 .4 Results wetted flow . . . . . . . . . . . . . 117

5.3.5 Results cavitating flow, $\sigma=0.68 \ldots \ldots . \ldots 123$

5.3 .6 Conclusions . . . . . . . . . . . . . . . 130

5.4 Conclusions on underlying flow mechanisms . . . . . . . . 132

6 Application Challenge 135

6.1 Introduction . . . . . . . . . . . . . . . . . . . . . . . 135

6.24119 DTRC Propeller . . . . . . . . . . . . . . . . . 137

6.2.1 Geometry . . . . . . . . . . . . . . . 137

6.2.2 Computational domain and mesh . . . . . . . . . . . 138

6.2 .3 Numerical parameters . . . . . . . . . . . . . . . . . . 139

6.2 .4 Results wetted flow . . . . . . . . . . . . . 141

6.2.5 Results for cavitating flow . . . . . . . . . . . . 151

6.2.6 Conclusions . . . . . . . . . . . . . . . . . . . . . 161

6.3 Leading Edge Skew Propeller . . . . . . . . . . . . . . . . 161

6.3.1 Geometry ................... . . 161

6.3.2 Computational domain and mesh . . . . . . . . . 163

6.3.3 Setting of the numerical solution parameters . . . . . . 163

6.3.4 Results wetted flow . . . . . . . . . . . . . 163

6.3.5 Results for cavitating flow . . . . . . . . . . . . . 170

6.3.6 Scaling . . . . . . . . . . . . . . . . . . . . . . . . . . . . . . . . . . . . . .

6.3.7 Conclusions . . . . . . . . . . . . . . . . 188

6.4 Conclusions . . . . . . . . . . . . . . . . . . . . . 188 
7 Conclusions and Recommendations 193

7.1 Numerical method . . . . . . . . . . . . . . . . . . . . . 193

7.2 Conclusions on results for wetted flow . . . . . . . . . . . . 194

7.3 Conclusions on results for cavitating flow . . . . . . . . . . . . 195

7.4 Recommendations for future study . . . . . . . . . . 196

$\begin{array}{ll}\text { Bibliography } & 197\end{array}$

Solution of cubic equation $\quad 205$

$\begin{array}{ll}\text { Acknowledgements } & 207\end{array}$

$\begin{array}{ll}\text { Curriculum Vitae } & 209\end{array}$ 


\section{Chapter 1}

\section{Introduction}

\section{$1.1 \quad$ Propeller}

Driven by the need of humans to travel by sea, propulsion concepts can be found in history since early times. Propulsion solution ideas date back as far as 220 BC by Archimedes and around 1500 by Leonardo da Vinci. Since then, the propulsor design progressed immensely, developing multiple solutions for ship propulsion. One of the most popular configurations is the screw propeller. In the beginning, its invention was controversial while many engineers were working on its concept around the end of XVIII century beginning of XIX century. Still, the invention of the propeller is attributed to Edward Shorter in 1802 who used a propeller (based on a previously developed concept, see [15]) powered by eight men on a capstan to move the transport ship Doncaster. Few decades later, in 1835, Francis Petit Smith and John Eriksson acquired patents for their screw propellers, marking the start of its contemporary development. Rotating bladed wheels, as well as twin-screw and single-screw installations and Archimedean screw propellers were the configurations build as propulsion devices. The familiar shape as a screw propeller was made by George Rennie's conoidal screw, patented in 1839. Despite their successes many implementation problems for screwpropelled ships had to be solved. However, it was not until the steam engine was invented that propellers became widely used, about 100 years ago.

Other existing propulsion units are ducted propellers, podded and azimuthing propulsors, contra-rotating propellers and waterjets. Just to mention some of the most known and used solutions of the modern times. More details on the propeller history, geometry and evolution of its design can be found in Carlton [15].

The purpose of a propeller is to produce thrust. Therefore, the performance 
characteristics, non-dimensional coefficients for thrust, torque and the efficiency are of main interest and specific for each design. Definitions for thrust and torque coefficients are as follows. For the thrust coefficient:

$$
K_{t}=\frac{T}{\rho n^{2} D^{4}}
$$

For the torque coefficient:

$$
K_{q}=\frac{Q}{\rho n^{2} D^{5}}
$$

The open water efficiency of a propeller is defined as:

$$
\eta_{o}=\frac{J K_{t}}{2 \pi K_{q}}
$$

where the advance ratio $J$ is defined as:

$$
J=\frac{V_{a}}{n D}
$$

In the above relations $\rho$ is the density, $n$ is the number of rotations per second of the propeller and $D$ is its diameter. The non-dimensional characteristics are valid for the model test experiments in the so-called open-water conditions. But unfortunately in real life the hull of the ship creates a complicated wake flow upstream of propeller and further more, also disturbances by manoeuvres. This complicated flow field, the environment in which the ship-propeller operates, influences drastically the operation of a propeller. Each propeller blade experiences a periodic fluctuation in flow velocity as it rotates in and out of parts of the wake. Such conditions typically result in cavitation in the tip vortex and on the propeller surface while cavitation would not occur for a uniform wake field. The appearance of cavitation leads to noise, vibrations and erosion of propeller blades as well as thrust reduction. All this issues make the propeller design quite difficult. Last century developments produced many propeller theories as well as accompanying empirical correction factors, to account for the actual operation in the marine environment. The non-uniform time-varying inflow velocity distribution is unknown precisely and therefore comparing experimental results with those of calculations is hard. But, in the last decade new types of computational methods have been developed, that may resolve this problem. Methodologies based on the Navier-Stokes equations, the most general description of continuum fluid motion, prove to be one of the most powerful fluid dynamics tool. 
However, their direct solution requires tremendous computer power and the present state-of-the-art permits only the flow at low Reynolds numbers to be computed. In reality, flows like propeller-hull computations, are flows at high-Reynolds numbers, and their direct numerical simulation (DNS) is unreachable for the moment. The practical solution of the Navier-Stokes equations is achieved by the Reynolds Averaged Navier Stokes (RANS) equations, which involve an approximation which necessitates the concept of turbulence models. The RANS approach is feasible as far as computing time is concerned and thought to be sufficiently accurate for industry applications, like the prediction of the propeller open water characteristics.

\subsection{Cavitation}

\subsubsection{History}

The use of a propeller at high velocities in the low pressure fields behind a ship, reveals new challenges for design when the cavitation phenomenon is triggered. Historically, cavitation was first reported by Sir Isaac Newton [62] and by L.P.Euler as appearing on a water wheel in 1754. In 1873, Osborne Reynolds [67] and [68] is the first that tried to explain the behaviour of ship propellers at high rotation speeds in a classic series of experiments by what we call today the ventilation phenomenon.

The basics of cavitation were introduced by Sir Charles Parson when he tested a series of propellers to assess the cavitation phenomenon for different designs. It all started with a performance problem in 1893 when trials of the destroyer HMS Daring reviled that the maximum speed was below its design speed of 27 knots. Based on the suggestion of William Froude, in 1895, Thornycroft and Barnaby [84] named the phenomenon of the breakdown of the performance of the propeller due to vapour presence: CAVITATION. Then, on a first turbine ship, called Turbina, Parsons [63] experienced similar difficulties and realized that:

"These cavities contained no air only vapour, and the greater portion of the power of the engine was consumed in the formation and maintenance of these cavities instead of the propulsion of the vessel."

Bad propeller performance led Parson to experiments on model scale, and by observing cavitation patterns he was able to produce a wide-bladed propeller design without cavitation. However, it required 9 propellers to drive 
the Turbina at 32 knots. From this point on, the cavitation on propellers became a widely investigated subject, experimentally and theoretically, and today is still a field with many uncertainties.

\subsubsection{Definition}

Cavitation is a fluid dynamics phenomenon that appears when a liquid changes into its vapor state due to a decrease of the pressure below the vapor pressure. Therefore, theoretically cavitation inception should occur when locally the pressure reaches the vapor pressure. However, in real life the formation of a cavity is influenced by many factors, as will be presented in the following.

Cavitation is present in many engineering systems like pumps, turbines and propellers. At locations where due to high velocities the pressure decreases, the liquid transforms into vapor. Cavitation can be recognized by its undesirable effects, like erosion, oscillations and vibrations, noise and reduction of the efficiency of the hydraulic units.

In 1980, Lauterborn [46] produced a classification scheme of the cavitation phenomenon presented in the figure 1.1.

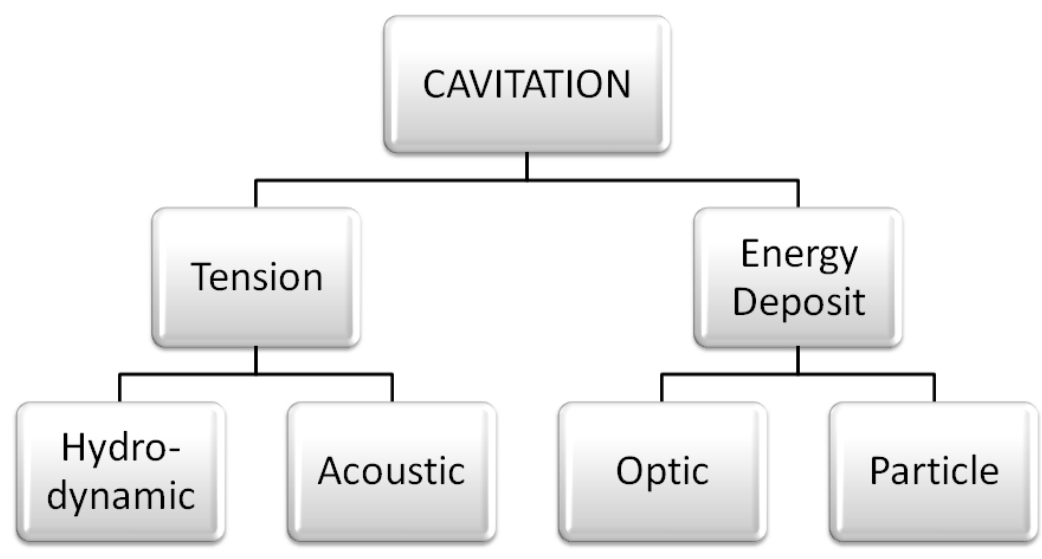

Figure 1.1: Cavitation classification scheme (Lauterborn, see [46])

Cavitation is produced in a liquid by high tension or by deposit of energy. The present study is dealing with the cavitation on propellers, therefore hydrodynamic cavitation achieved by tension exerted on the liquid. Hydrodynamic 
cavitation occurs in devices that introduce a pressure decrease in the liquid that eventually generates tension.

\subsubsection{Stressing of liquids}

The vapor pressure of liquids depends on the temperature, i.e. the vapor pressure increases with temperature. Therefore cavitation is defined when for given temperature the pressure drops below the vapor pressure. In contrast, the boiling phenomenon occurs when for given pressure the temperature increases up to the point that the vapor pressure becomes equal to this given pressure. When the liquid tension reaches a certain value, the liquid breaks or cavitates and the fluid becomes a biphasic system formed by liquid and vapor.

Let us consider a typical water phase diagram presented in figure 1.2.

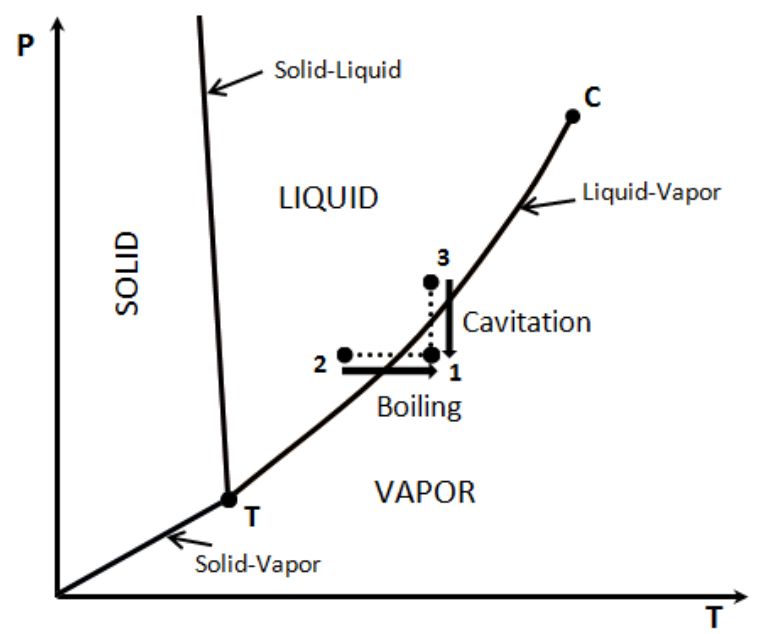

Figure 1.2: Typical water phase diagram

Two types of vaporization are possible, first the one well known due to the temperature increasing from point 2 to point 1 at constant pressure. Along the liquid-vapor curve, liquid and vapor can coexist. The second way of reaching the vapor state from a liquid phase is from point 3 to point 1, by means of reducing pressure at constant temperature. This case requires the creation of cavities within the fluid itself, and depends therefore on the fluid 
properties. If water contains numerous quantities of dissolved air/nuclei, then the cavities can be formed at higher pressure than the vapor pressure. Also, if there are no nuclei, the liquid can withstand a high negative tension without cavitating. This tensile strength of the liquid is highly dependent on the quality of the liquid and on the value of the critical tension, thus requires prior knowledge of nuclei content. The nuclei content is a critical point in the prediction of cavitation. Despite the extensive literature on the subject only two principal models of nucleation are fully developed: the stationary crevice model and the entrained nuclei model, both described in [11]. The stationary nuclei are assumed to exist in the small crevices of the solid wall, and the entrained nuclei are assumed to travel with the liquid. Usually the main source of nuclei is considered to be the entrained flow.

\subsubsection{Cavitation number}

Considering Bernoulli's equation, the pressure variation along the surface of a body in a steady, incompressible inviscid flow, neglecting effects of gravity, is given by:

$$
p_{1}+\frac{1}{2} \rho u_{1}^{2}=p_{2}+\frac{1}{2} \rho u_{2}^{2}=\text { constant along streamlines }
$$

In equation $1.5, u$ is the velocity and $p$ is the pressure.

The cavitation number $\sigma$ is defined by dividing difference between the local static pressure and the vapor pressure, $p_{0}-p_{v}$, by the dynamic pressure of the flow:

$$
\sigma=\frac{p_{0}-p_{v}}{\frac{1}{2} \rho u^{2}}
$$

Where $p_{0}$ is the ambient static pressure, $p_{v}$ is the temperature dependent the vapour pressure and $\frac{1}{2} \rho u_{0}^{2}$ is the dynamic pressure of the free stream. When $\sigma$ is reduced to the value where cavitation is first observed, is called the incipient cavitation number $\sigma_{i}$. Further reduction of the cavitation number increases the formation of vapor bubbles and developed cavitation appears, such as sheet cavitation. Moreover, if cavitation occurs when the pressure reaches the vapor pressure results in:

$$
\sigma=-C_{p \min }
$$

Where $C_{p m i n}$ is the minimum value of the pressure coefficient $C_{p}$ and can be 
determined by experiment or by computation of single-phase flow using:

$$
C_{p}=\frac{p-p_{0}}{\frac{1}{2} \rho u_{0}^{2}}
$$

Where $p_{0}$ and $u_{0}$ are the pressure and velocity of the upstream flow. Moreover, unfortunately many factors influence relation 1.7, like the presence or the absence of nuclei, the tension, the time needed for the bubble to be visible and the fact that there are also uncertainties in the measurements. Nevertheless this assumption is useful as an initial guess and as a start in the cavitation analysis. The cavitation number $\sigma$ is also providing a measurement tool for the degree of cavitation present within a liquid. For a high value of $\sigma$ the reference pressure $p_{0}$ is high and the pressure will be above vapor pressure everywhere. Therefore the fluid will stay free of cavitation. Decreasing the reference pressure $p_{0}$ the incipient cavitation number is reached and continuing to lower the $p_{0}$ value the cavitating area is growing larger and larger, eventually reaching the state of so-called super cavitation. If the reference pressure is increased, the cavitation will start to disappear but usually for a value higher than the incipient value, called desinent value. Therefore a hysteresis effect is often observed when decreasing and increasing the pressure around the values that the caviation number equals the incipient value.

\subsection{Propeller cavitation}

\subsubsection{Cavitation types}

Behind the ship, a working propeller can experience all types of cavitation, see the sketch from Kinnas 1996 and reproduced in figure 1.3. Cavitation patterns can be classified in 3 groups, though on a propeller combinations of different types are usually occurring. Cavitation appearances can mainly be: stationary, with respect to the propeller, such as sheet cavitation; and travelling, such as bubble cavitation and finally vortex cavitation (see figure 1.4 as an example of tip vortex cavitation). Secondary effects, such as: interactions between cavities (see figure 1.5 as an example of leading edge-tip vortex cavitation), re-entrant jets, turbulence and the interface instabilities, to name the most frequent factors, do make cavitation analysis even more complicated. 


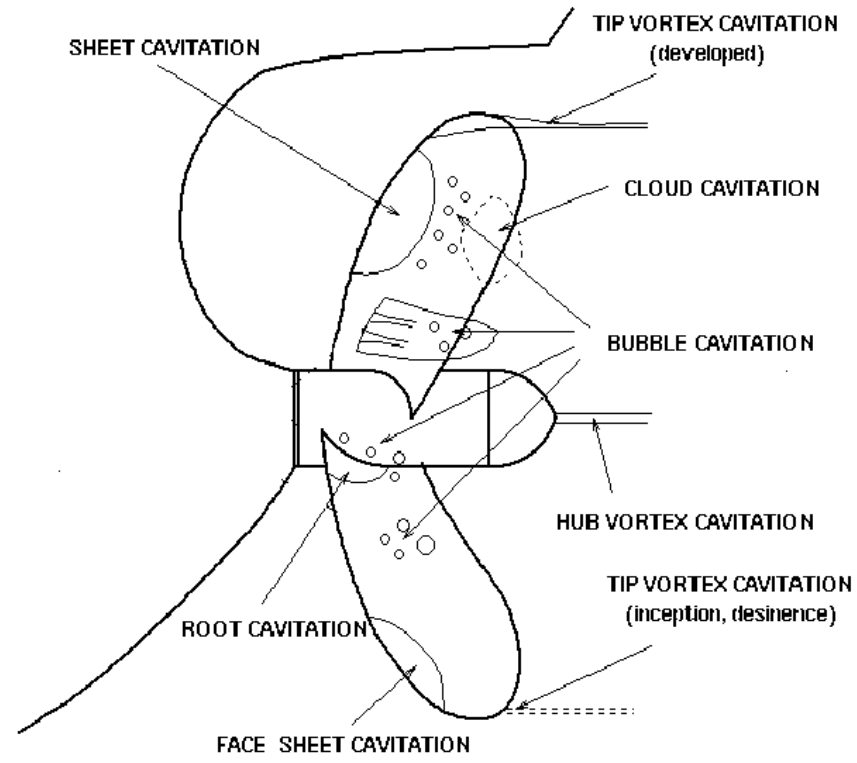

Figure 1.3: Cavitation types on propeller, from S.A.Kinnas 1996

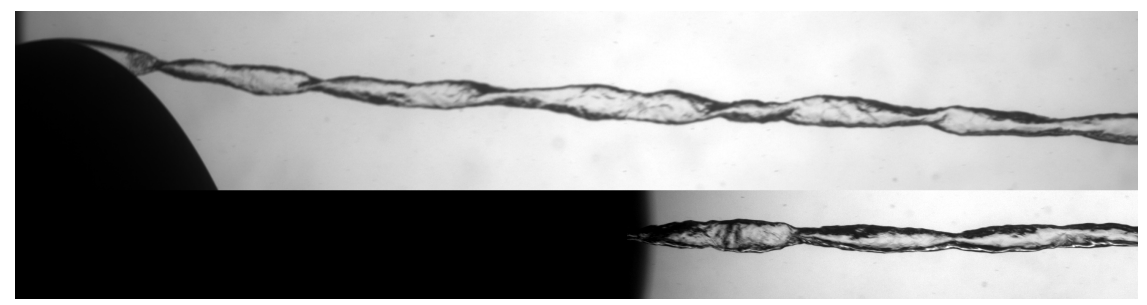

Figure 1.4: Tip vortex cavitation, from [64]

Propeller configurations are widely used to propel ships and it is very important to have low levels of noise and vibrations (e.g. for passengers comfort), while erosion is important with respect to the degradation of the performance of the ship (delivered propeller thrust). Therefore, when defining design requirements, cavitation which affects the lifetime of the propeller and related issues, like noise, vibrations and erosion are addressed. Another issue of the working propeller is the operating condition in the ship wake which is usually 


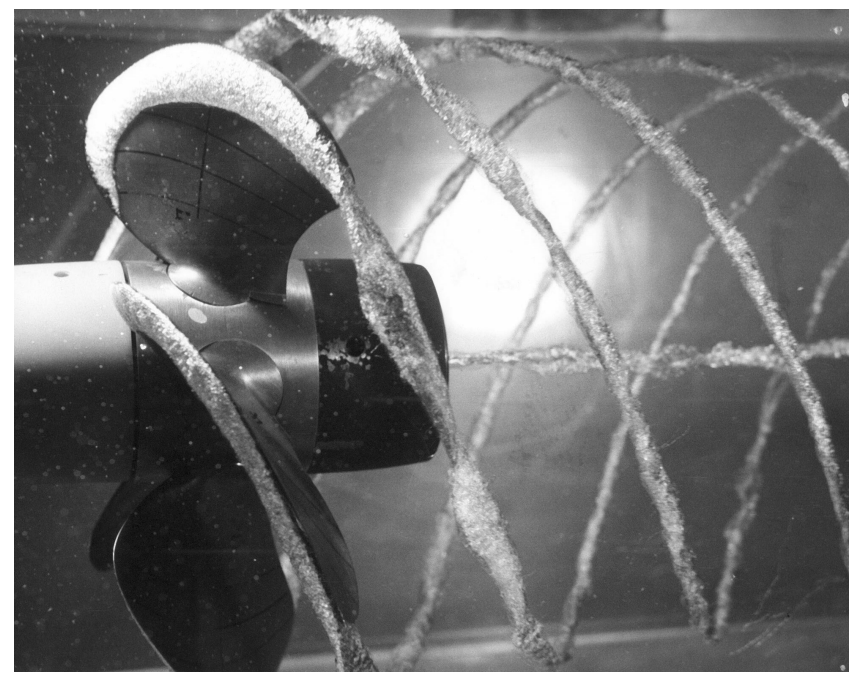

Figure 1.5: Leading edge-tip vortex cavitation, from [26]

unknown and difficult to scale in experiments without all kinds of corrections which are subject to considerable uncertainty.

In the region of the propeller blade tip, at high Reynolds number, swirling flow regions with high vorticity and low pressure are formed. Usually cavitation inception occurs in these vortices. This type of cavitation develops further, filling the entire vortex core with vapor. This type of cavitation within a vortex core, starting close to the propeller blade tip, is called tip vortex cavitation and is the subject of the present thesis. For a ship propeller, cavitation tends to occur first within the core of the tip vortex and tip vortex cavitation often forms prior to other types of cavitation. This makes the inception and the development of propeller tip vortex cavitation very important already in the design stage.

\subsubsection{Tip vortex cavitation}

During the design phase of the propeller, tip vortex cavitation raises difficulties when its prediction through experiments is hindered due to the complexity of scaling the cavitation inception number. In [54], McCormick establishes the basis of the scaling of tip vortex cavitation inception. After numerous model tests on propellers, he derived an empirical expression for the tip vor- 
tex cavitation inception number at full scale based the cavitation inception number determined on model scale and the Reynolds numbers corresponding to the model scale and full scale. This scaling rule for cavitation inception number is:

$$
\frac{\sigma_{F S}}{\sigma_{M S}}=\left(\frac{R e_{F S}}{R e_{M S}}\right)^{0.35}
$$

The Reynolds number $R e$ is defined as:

$$
R e=\frac{n D^{2}}{\nu}
$$

where $\nu$ is kinematic viscosity.

In equation 1.9, subscripts FS and MS refer to full scale and model scale, respectively.

Equation 1.9 is widely used today by model test basins. But, based on experience, most of the testing institutes calibrate this relation with the properties their specific facility. For the exponent, 0.35 , in equation 1.9 values ranging from 0.25 to 0.4 can be found, for example in [76] a exponent of 0.4 is used. However, the relation in equation 1.9 is not sufficient to assess the tip vortex cavitation inception at full scale. Numerous propellers that suffer from this type of cavitation and its nuisance confirm this. Also, model tests of tip vortex cavitation inception are, if existent, a design check and not a design tool. Therefore more investigation is needed into the subject to fully understand and assess the characteristics of the formation of tip vortex cavitation and its effects, for application in the propeller design phase.

\subsubsection{Cavitation nuisance}

For propellers, cavitation is often a problem because of the collapse of vapor clouds and bubbles. During the collapse of a bubble very large liquid-vapor interface velocities and localized pressures occur. Due to this process, cavitation is generating noise and vibrations. The most serious problem, however, is material damage caused by cavitation bubbles that collapse close to or on the surface of propeller blades. Cavitation damage is a highly investigated subject but, due to the fact that is an unsteady flow phenomenon, dependent on the particular material, many of uncertainties and questions regarding the fundamental mechanics exist. Many empirical rules exist to help the propeller designer but still not sufficient to understand the complicated process of cavitation. The question whether cavitation damage is 
caused by micro jets or by shock waves generated when the bubbles collapse is still open. Whatever the answer the phenomenon of cavitation damage is due to repeated collapse and causes local fatigue failure of the material, so that pieces of material detach from the surface. Thus, cavitation damage is a fatigue failure with a crystalline appearance (as from [12] and [11]), involving complex mechanics. Bubble collapse can also generate noise and vibrations as a consequence of the high pressures that are generated when the bubbles are highly compressed and subsequently propagated into the flow field.

In particular, as the main topic of the present thesis, tip vortex cavitation produces noise and vibration, but has apparently no influence on the performance of the propeller. Due to its location at the tip of the blade, tip vortex caviation collapse on the blade is rare. However, tip vortex cavitation can damage the rudder if present behind the propeller. Therefore, tip vortex cavitation prediction is most important for ships that should be free of noise and vibrations, like navy ships, cruise ships, medical and research vessels and for propeller-rudder configurations.

\subsection{Motivation and Objectives}

The case of an open propeller is the most widely used case of studying the propulsion of ships. Propeller design has to meet many requirements, most of them imposed by the ship owner. But, from the hydrodynamic point of view the most important requirement is the allowed extent of cavitation and cavitation pattern. Therefore, one of the most important steps in the design stage of a propeller is to predict cavitation phenomena.

Since the early times, the checking of a propeller design was by representing the full scale situation of the propeller working on a ship, in a model scale experiment. Today, this is still a much used procedure to check a propeller design on performance and on cavitation characteristics. The experimental approach is fully developed and has reached maturity. But, many uncertainties due to Reynolds number scaling effects exist when dealing with tip vortex cavitation. For more insight into the experimental approach, Kuiper [43] investigated three typical designed propellers for each type of cavitation (sheet, bubble and vortex) and uses model testing to analyse these cavitation phenomenon.

Cavitation has many appearances but, the most distinctive ones are bubble, sheet and vortex cavitation. Some cavitation types like bubble and sheet cavitation can be reproduced at model scale or calculated with inviscid flow methods quite well. However, experimental prediction of tip vortex cavitation 
at model scale is difficult due to scaling effects. In addition, the cavitation inception value on model scale needs to be scaled to full scale predictions. Moreover, tip vortex cavitation inception in model tests is very dependent on nuclei present in the basin and on propeller roughness, thus facility dependent. Full scale predictions based on experimental values make use of the McCormick scaling rule [54] developed in the 60's, see equation 1.9. This procedure is not very reliable and the desire for an accurate procedure to predict tip vortex cavitation inception and development on full scale, already in the design stage is imperious.

Because of tip vortex cavitation, cruise vessels, ferries and navy ships, often suffer undesirable effects like noise, vibrations and erosion. Thus, one of the most important achievements of a propeller design is that the propeller is free of tip vortex cavitation. Prediction and quantification of tip vortex cavitation is a valuable asset for a propeller designer.

The goal of the thesis is to accurately determine the inception and development of tip vortex cavitation on marine propellers, by means of a CFD method which might lead to better propeller designs.

\subsection{Approach}

Design of a propeller requires accurate knowledge of the distribution of the velocity and pressure on the surface of the propeller blades, on full scale and behind a ship, ideally. In order to acquire this knowledge, different methods have been developed. Two major ways of analysing the performance of a propeller can be distinguished: practical/experimental methods and theoretical/computational methods. The experimental approach, that began with Parson, is in its mature phase, while the computational approach, based on high-fidelity models is increasingly utilised.

Propeller theories started being utilised during the last 60 years, resulting in a wide variety of design and analysis methods for predicting propeller performance. For more details on mathematical models for propellers, [15] offers a very good overview. These methods vary from basic theories to the most complex mathematical descriptions of flow dynamics.

In the last decades Computational Fluid Dynamics (CFD) is becoming very popular due to advances in numerical algorithms and the increase in computer power. In its most complex form CFD employs the Navier Stokes equations to resolve the fluid motion. The Navier-Stokes flow model is based on a set of partial differential equations of second order, derived in the 1840s on the basis of conservation laws. For flows at low Reynolds numbers and for 
simple geometries, it is often possible to find approximate solutions to the Navier-Stokes equations. However, for practical applications, direct solutions of the Navier-Stokes equations is not an option and the practical approach is provided by the Reynolds-Averaged Navier Stokes equations (RANS). However, the time-averaging of the Navier Stokes equations introduces new unknown terms. This terms are expressed in terms of time-averaged flow field quantities by turbulence models. The path used in the current thesis to assess the inception and development of tip vortex cavitation on marine propellers is using a computational method solving the RANS equations. Details of the RANS method can be found in the Mathematical Modeling chapter.

Numerical simulations for propellers using the RANS approach without cavitation modelling are already widely used in industry. Rhee and Josi [69] use numerical simulations for propeller P5168 and validate their results with the experimental data of Chesnakas and Jessup [22]. Their computational results are in line with those of the experiments. However, the torque and thrust are over-predicted with 11 and 8 percent, respectively, still promising results. In the same line are most of the RANS calculations for propellers reported in [49], of the LEADING EDGE EU project and ITTC for performances predictions using CFD. Therefore, RANS simulations for propellers start to give results comparable to results from experiment.

To detect a tip vortex in the flow around a propeller blade using a CFD approach is not a trivial task. Already the definition of the vortex is complex. The detection of vortices is a highly investigated subject not only for propellers, also for hydrofoils and aircraft wings. Most of the predictions of tip vortices employing CFD can be found within the aerospace field for the case of vortices generated by wings and rotors. Predictions of the tip vortex have been performed by Ghias et al. [32] for a wing with a NACA 2415 section. Using LES, they achieve very good results in the prediction of the distribution of the velocity and vorticity within the vortex. For propeller blades, tip vortex prediction has been addressed within the LEADING EDGE EU project and recently by Lifante [51]. All results locate and predict regions with low pressure and swirling velocity fields but their accuracy is low due to too coarse meshes and approximations associated with the turbulence models used.

To accurately determine and understand cavitation inception, development of cavitation and collapse of vapor regions requires much more work. Numerical simulations of cavitation have been performed by Abdel-Masksoud [6] using the CFX-TASCflow RANS solver to assess the cavitation behaviour of a propeller. Inception and location of cavitation are well predicted but prediction of its development is not yet achieved. Main concerns and difficulties of the CFD calculations for the wetted flow are the grid requirements 
and turbulence modelling. For the cavitating flows the issue of cavitation modeling adds an extra dimension to the problem.

Therefore, trying to predict tip vortex cavitation involves two difficult tasks, first an accurate prediction of the tip vortex and secondly an adequate modeling of the cavitation phenomenon. This is a complex problem, not easy to solve requiring a detailed investigation into tip vortex detection and cavitation modeling.

Numerical simulations of cavitating tip vortices have been performed at Dynaflow by Chahine, Hsiao et al., see [76] and [23]. They use a bubble dynamics model and URANS to predict cavitation inception and tip vortex flows for complex configurations. Their results are very successful in the prediction of inception as well as analysing scaling effects for tip vortex cavitation.

In conclusion, the prediction of tip vortex cavitation on propellers is a challenging task with many uncertainties and questions, questions addressed in the present thesis. The present thesis attempts to answer and clarify the issues of these complicated numerical simulations by exploring the limits of employing the RANS method. CFD can bring the prediction of tip vortex cavitation to a new level, which is the goal of the present study. To achieve this goal, CFD calculations will be carried out for different propeller shapes for conditions that exhibit tip vortex cavitation. For the considered propellers detailed experimental data is available for the tip vortex flow. The capability of RANS to predict concentrated vorticity fields in the propeller tip vortex and the associated requirements on the grid, turbulence model and cavitation model will be addressed. The results will be investigated in detail, with a focus on turbulence modelling and meshing strategies.

The thesis concludes with an assessment of the use of a RANS approach in propeller design with emphasis on designing for the minimal cavitating vortex. 


\subsection{Outline thesis}

Following the present Introduction chapter the thesis is structured as outlined below.

The thesis uses numerical simulations to assess cavitation, therefore the governing flow equations including the turbulence model and the cavitation model are described in the second chapter: Mathematical Modeling.

Vortex cavitation is of main interest in the simulations, therefore the third chapter, Vortex Detection and Visualisation, deals with vortex definition and the existing methods available to determine vortical structures numerically. This chapter correlates with a brief description of the selected method used in the thesis when dealing with wetted or cavitating flows with vortices.

Chapter four is dealing with the uncertainty study of the developed numerical method for steady and unsteady flow conditions. Guidelines for the grids and simulation time step are also detailed in this chapter.

Table 1.1 summarises the main validation material available for the test cases dealing with wetted or cavitating vortices.

\begin{tabular}{|c|c|l|l|l|}
\hline Flow type & Validation Data & $\begin{array}{l}\text { Elliptic 11 } \\
\text { rake hydro- } \\
\text { foil }\end{array}$ & $\begin{array}{l}4119 \\
\text { DTRC } \\
\text { propeller }\end{array}$ & $\begin{array}{l}\text { LE Skew } \\
\text { propeller }\end{array}$ \\
\hline wetted & Velocity and pressure fields & - & $\sqrt{ }$ & - \\
\hline wetted & Tip vortex core & - & $\sqrt{ }$ & - \\
\hline cavitating & Cavitation inception & - & $\sqrt{ }$ & $\sqrt{ }$ \\
\hline cavitating & Tip vortex core & $\sqrt{ }$ & - & - \\
\hline cavitating & Cavity visualisation & - & - & $\sqrt{ }$ \\
\hline
\end{tabular}

Table 1.1: Validation material for tip vortices in wetted and cavitating flow

After describing the governing equations, defining the vortex structure and verifying the method, the results obtained for selected test cases address underlying flow characteristic and application challenges, in chapter 5 and 6 , respectively.

Chapter 5 considers two test cases: the Twist 11 hydrofoil and the Elliptic 11 rake hydrofoil. Both test cases are analysed in wetted and cavitating flow conditions and guidelines for turbulence modeling are given.

Chapter 6 presents the results obtained for two benchmark propellers: the 4119DTRC Propeller and the Leading Edge Skew Propeller. Wetted and cavitating flow conditions are investigated at model scale. For the Leading Edge Skew Propeller, also the scaling of the performance and the tip vortex is investigated. Conclusions are formulated on the capability of the method 
to predict tip vortex cavitation inception and tip vortex development, and the use of the method in the design stage of propellers.

The last chapter, discusses the main conclusions of the thesis and gives recommendations for further study on tip vortex cavitation. 


\section{Chapter 2}

\section{Mathematical modeling}

\subsection{Conservation Principles}

\subsubsection{Introduction}

Physical aspects of the fluid motion are governed by general conservation principles. Ones these principles are known, they can be particularised and applied to conserved quantity like mass, momentum and energy.

Before determining the general conservation equation, a few definitions have to be introduced: the control volume, the bounding surface of the control volume and the concepts of extensive and intensive quantity.

A control volume $(\tau)$ is a region in $3 \mathrm{D}$ space determined by a bounding surface impermeable or permeable to mass, momentum, energy. The fluid within such a control volume is subjected to forces volumetrically or through its bounding surface. The bounding surface of the control volume is the surface $(\sigma)$, i.e. $\sigma=\partial \tau$.

An extensive quantity $(E)$ within a control volume $\tau$ is any quantity that doubles its value when two identical volumes at identical conditions are added. Examples of extensive quantities are: mass, length, volume, entropy and energy. If a quantity does not change its value when two identical volumes at identical conditions are added, and remains constant, then this quantity is called intensive $(e)$. As example of $e$ : density, temperature, pressure and viscosity.

For most of the extensive quantities it is possible to associate a corresponding intensive quantity. In this case, the intensive quantity $e$ represents the volume density of the associated extensive quantity $E$. This relation can be written mathematically as: 


$$
E(t)=\int_{\tau} e(t, \vec{x}) d \tau
$$

Note that the conservation laws are applied only to extensive quantities like: mass, momentum and energy.

The general conservation law applicable to any extensive quantity $E$ is determined in the next section, as from [25].

\subsubsection{General Principle of Conservation}

Let us consider a control volume $\tau$ bounded by the closed surface $\sigma=\partial \tau$, and $E$, the extensive quantity corresponding to this volume. We ask ourselves: if we know the value of $E$ at a certain moment in time, how does this quantity change in time?

First we have to acknowledge that there are two types of dependencies: (1) The production and destruction of the quantity, called source $P$ of the extensive quantity $E$. Production / destruction can be found in the volume but also at its surface. And (2), the changes due to fluid passing through the surface $\sigma=\partial \tau$, called the flux $\phi$ of the extensive quantity $E$ into or out of the control volume $\tau$.

Now it is possible to answer the question and state that: the rate of change of the quantity $E$ in time can be written mathematically as a summation of the sources and changes of the extensive quantity $E$, as:

$$
\frac{d E}{d t}=P[E]+\phi[E]
$$

Moreover, the production of an extensive quantity related to a control volume, can be interior or on the surface. Therefore we can associate two intensive quantities for production, one related to each point inside the volume as:

$$
P_{\tau}[E]=\int_{\tau} q_{\tau}^{E}(t, \vec{x}) d \tau
$$

and a second one related to production at points at the surface as:

$$
P_{\sigma}[E]=-\int_{\sigma}{\overrightarrow{q_{\sigma}}}^{E}(t, \vec{x}) \cdot \vec{n} d \sigma
$$

Where ${\overrightarrow{q_{\sigma}}}^{E}$ is the flux at the surface. Here $\vec{n}$ is the external unit normal to the surface $\sigma=\partial \tau$. 
From equations 2.3 and 2.4 , the source $P$ can be written as:

$$
P[E]=\int_{\tau} q_{\tau}^{E}(t, \vec{x}) d \tau-\int_{\sigma}{\overrightarrow{q_{\sigma}}}^{E}(t, \vec{x}) \cdot \vec{n} d \sigma
$$

The flux $\vec{J}^{E}$ of $E$ is a surface quantity and represents the transfer of quantity $E$ between the control volume and its surroundings and can be written as:

$$
\phi[E]=-\int_{\sigma} \overrightarrow{J^{E}}(t, \vec{x}) \cdot \vec{n} d \sigma
$$

An analysis into the transfer phenomena at the permeable interface of a control volume and its surrounding shows two processes: one convective, due to the macroscopic movement of the fluid and one diffusive due to the submacroscopic movement. Therefore the flux vector $\overrightarrow{J^{E}}$ can be divided in two components, a convective flux and a diffusive flux written as:

$$
\overrightarrow{J^{E}}=\overrightarrow{J_{C}^{E}}+\overrightarrow{J_{D}^{E}}
$$

Where $\overrightarrow{J_{C}^{E}}$ is the convective component of the flux and $\overrightarrow{J_{D}^{E}}$ is the diffusive component.

Since $e$ is the volume density of quantity $E$, and considering $\vec{v}$ the velocity of the macroscopic transfer of quantity $E$, the convective flux can be written as:

$$
\overrightarrow{J_{C}^{E}}=-\int_{\sigma} e(t, \vec{x}) \vec{v} \cdot \vec{n} d \sigma
$$

And the diffusive component can be written as: $\overrightarrow{J_{D}^{E}}=-\int_{\sigma} \overrightarrow{j_{D}^{E}} \vec{n} d \sigma$.

Note that, the diffusive flux is not the objective of fluid mechanics and in general is given by semi-empirical constitutive laws.

Finally, gathering all the contributions we can rewrite equation (2.2) as:

$\frac{d}{d t} \int_{\tau} e(t, \vec{x}) d \tau=\int_{\tau} q_{\tau}^{E}(t, \vec{x}) d \tau+\int_{\sigma}{\overrightarrow{q_{\sigma}}}^{E}(t, \vec{x}) \cdot \vec{n} d \sigma-\int_{\sigma} e(t, \vec{x}) \vec{v} \cdot \vec{n} d \sigma-\int_{\sigma} \overrightarrow{j_{D}^{E}}(t, \vec{x}) \cdot \vec{n} d \sigma$

where $\vec{v}$ is the relative velocity with respect to the bounding surface $(\sigma)$ and $\vec{v}=\vec{V}-\vec{V}_{\sigma}$ with $\vec{V}$ the velocity of the fluid and $\vec{V}_{\sigma}$ the velocity of the bounding surface of the control volume.

Equation (2.9) represents the general transport equation.

Considering the divergence theorem $\left(\int_{\sigma} e \vec{V} \cdot \vec{n} d \sigma=\int_{\tau} \nabla \cdot(e \vec{V}) d \tau\right)$ and Leibniz's rule $\left(\frac{d}{d t} \int_{\tau} e(t, \vec{x}) d \tau=\int_{\tau} \frac{\partial e(t, \vec{x})}{\partial t} d \tau+\int_{\sigma} e \vec{V}_{\sigma} \cdot \vec{n} d \sigma\right)$, we can rewrite 
equation (2.9) as:

$$
\int_{\tau}\left[\frac{\partial e(t, \vec{x})}{\partial t}-q_{\tau}^{E}(t, \vec{x})+\nabla \cdot\left({\overrightarrow{q_{\sigma}}}^{E}(t, \vec{x})+e(t, \vec{x}) \vec{V}+\overrightarrow{j_{D}^{E}}(t, \vec{x})\right)\right] d \tau=0
$$

Equation (2.9) is the integral form of the transport theorem, and the local form of the transport theorem which follows from equation (2.10) because $\tau$ is an arbitrary control volume, is:

$$
\frac{\partial e(t, \vec{x})}{\partial t}-q_{\tau}^{E}(t, \vec{x})+\nabla \cdot\left(\overrightarrow{q_{\sigma}^{E}}(t, \vec{x})+e(t, \vec{x}) \vec{V}+\overrightarrow{j_{D}^{E}}(t, \vec{x})\right)=0
$$

for all $\vec{x} \in \tau$.

Using equation 2.11 it is possible to obtain all the equations of continuum fluid mechanics. In the following subsections this equation is considered for conservation of mass, momentum and energy.

\subsubsection{Mass Conservation}

When the extensive quantity is mass $m$ and the corresponding intensive quantity is the density $\rho$, equation 2.11 becomes:

$$
\frac{\partial \rho}{\partial t}-q_{\tau}^{m}+\nabla \cdot\left({\overrightarrow{q_{\sigma}}}^{m}+\rho \vec{V}+\vec{j}_{D}^{m}\right)=0
$$

In case there are no chemical reactions, or phase transitions, i.e. there are no mass sources:

$$
q_{\tau}^{m}=0, \overrightarrow{q_{\sigma}}{ }^{m}=\overrightarrow{0}
$$

The diffusive mass flux can be considered zero, because we assume the composition of the fluid to be fixed.

$$
\vec{j}_{D}^{m}=\overrightarrow{0}
$$

Then we find the conservation form of the continuity equation:

$$
\frac{\partial \rho}{\partial t}+\nabla \cdot(\rho \vec{V})=0
$$

Equation 2.15 represents the mass conservation principle in the conservation PDE form.

In case of cavitation, there is a phase transition from liquid to vapor or vice versa. In that case, equation 2.15 will have a non-zero right-hand side, see section 2.3.5. 


\subsubsection{Momentum Conservation Principle}

When the extensive quantity is the total impulse $\vec{I}$ of the control volume $\tau$ and the corresponding intensive quantity is $\rho \vec{V}$, the equation 2.1 becomes:

$$
\vec{I}=\int_{\tau} \rho \vec{V} d \tau
$$

Now equation (2.11) becomes:

$$
\frac{\partial(\rho \vec{V})}{\partial t}-q_{V}^{\vec{I}}+\nabla \cdot\left[\overline{\bar{q}}_{S}^{\vec{I}}+(\rho \vec{V}) \otimes \vec{V}+\overline{\bar{j}}_{D}^{\vec{I}}\right]=\overrightarrow{0}
$$

If we consider $\vec{f}$, the exterior force density per unity mass we can write:

$$
q_{V}^{\vec{I}}(t, \vec{x})=\rho \vec{f}
$$

In the same way, the impulse sources at the surface will be represented by the forces distributed on the surface:

$$
\overline{\bar{q}}_{S}(t, \vec{x})=-\overline{\bar{\sigma}}
$$

Where $\overline{\bar{\sigma}}$ is the stress tensor at the surface $\sigma$, the minus sign corresponds to the convention of the surface normal. Again the diffusive flux is considered zero, and we can rewrite equation 4.19 as:

$$
\frac{\partial(\rho \vec{V})}{\partial t}-\rho \vec{f}+\nabla \cdot[-\overline{\bar{\sigma}}+(\rho \vec{V}) \otimes \vec{V}]=\overrightarrow{0}
$$

To express the stress tensor, the Stokes postulates are used to relate the spatial gradient of the velocity field to the stress field, and the mathematical expression for a Newtonian fluid is:

$$
\overline{\bar{\sigma}}=2 \mu \overline{\bar{d}}+(-p+\lambda \nabla \cdot \vec{V}) \overline{\bar{I}}
$$

In the literature, the viscous part of the stress tensor $\overline{\bar{\tau}}$ is defined as:

$$
\overline{\bar{\tau}}=2 \mu \overline{\bar{d}}-\frac{2}{3} \mu(\nabla \cdot \vec{V}) \overline{\bar{I}}
$$

Using equation 2.22 the stress tensor $\overline{\bar{\tau}}$ then becomes:

$$
\overline{\bar{\sigma}}=\overline{\bar{\tau}}-p \overline{\bar{I}}
$$


In equation (2.21), $\overline{\bar{d}}$ is the rate of strain tensor defined as:

$$
\overline{\bar{d}}=\frac{1}{2}\left[\vec{\nabla} \vec{V}+(\vec{\nabla} \vec{V})^{T}\right]
$$

In equation (2.24), $p$ is the static pressure, $\overline{\bar{I}}$ is the second-order unit tensor, $\mu$ is the molecular dynamic viscosity and $\lambda$ is the second viscosity coefficient. Using Stokes hypothesis that the trace of $\overline{\bar{\tau}}$ should be zero, leads to $\lambda=-\frac{2}{3} \mu$. Now, the momentum equation 2.20, using equation 2.23 becomes:

$$
\frac{\partial}{\partial t}(\rho \vec{V})+\vec{\nabla} \cdot(\rho \vec{V} \otimes \vec{V})=\rho \vec{f}-\vec{\nabla} p+\vec{\nabla} \cdot \overline{\bar{\tau}}
$$

the conservation PDE form.

Equation 2.25 in known as the momentum or Navier-Stokes equation and represents the conservation principle of momentum.

\subsubsection{Energy Conservation Principle}

The extensive quantity is the total energy, $E$, of the fluid. $E$ is the sum of the internal energy per unit of mass, $e$, and the kinetic energy per mass unit, $|\vec{V}|^{2} / 2$, so that $E=e+\frac{\overrightarrow{V^{2}}}{2}$ then, the general conservation principle from equation 2.11 takes the form of:

$$
\frac{\partial(\rho E)}{\partial t}-q_{V}^{E}+\nabla \cdot\left(\overrightarrow{q_{S}^{E}}+\rho E \vec{V}+\vec{j}_{D}^{E}\right)=0
$$

The first law of thermodynamics states that the variation of the energy is equal to the summation of the amount of heat supplied to the system and the work done on the system. Therefore the volume sources are the sum of the work done, per unit in time, by the external forces $(\rho \vec{f} \cdot \vec{V})$ and the heat added volumetrically $\left(q_{V}\right)$ :

$$
q_{V}^{E}=\rho \vec{f} \cdot \vec{V}+q_{V}
$$

Surface sources are represented by the mechanic work of the tensions as:

$$
\vec{q}_{S}^{E}=\overline{\bar{\sigma}} \vec{V}
$$


The diffusive flux is usually given by Fourier's law of heat conduction:

$$
\vec{j}_{D}^{E}=-k \nabla T
$$

Where $k$ is the thermal conductivity coefficient and $T$ is the absolute temperature.

Finally, equation 2.26 becomes:

$$
\frac{\partial(\rho E)}{\partial t}+\nabla \cdot(\rho E \vec{V})=\nabla \cdot(k \nabla T)+\nabla \cdot(\overline{\bar{\sigma}} \vec{V})+\rho \vec{f} \cdot \vec{V}+q_{V}
$$

Equation 2.30 is the conservation of energy in PDE conservation form.

The conservation principles are the basis of the fluid dynamics, more details can be found in [18], [90] and [28].

The conservation equations of mass (equation 4.17), momentum (equation 2.25 ) and energy (equation 2.30) form a system of 5 transport equations. Knowing the volumetric force field and the volumetric heat source they contain 7 unknowns $(p, \rho, e, T, \vec{u})$. The system of equations is closed utilising the thermodynamic state principle: for a fluid of fixed composition the thermodynamic state is fixed by specifying two thermodynamic variables.

Thus equations of conservation of mass, momentum and energy form the system of Navier-Stokes equations and together with the equations of state and the empirical laws for the viscosity coefficient and thermal conductivity, represent the most complete description of the fluid motion. This system of seven equation governs the physical aspects of the fluid flow.

\subsection{Mathematical models}

\subsubsection{Navier-Stokes equations}

The Navier-Stokes equations are the most important fluid dynamic system of equations since these conservation equations determine and characterise all fluid motions. Therefore, in the following, different forms of the NavierStokes equations are presented and a few comments added.

Mathematically these different forms are equivalent, but from a perspective of numerical methods different forms may require different discretizations.

In vector form the Navier-Stokes equations is: 
-mass conservation equation:

$$
\frac{\partial \rho}{\partial t}+\nabla \cdot(\rho \vec{V})=0
$$

-momentum conservation equations:

$$
\frac{\partial \rho \vec{V}}{\partial t}+\nabla \cdot[(\rho \vec{V}) \otimes \vec{V}]=\rho \vec{f}-\nabla p+\nabla \cdot \overline{\bar{\tau}}
$$

-energy conservation equation:

$$
\frac{\partial(\rho E)}{\partial t}+\nabla \cdot(\rho H \vec{V})=\rho \vec{f} \cdot \vec{V}+q_{v}+\nabla \cdot(k \nabla T)+\nabla \cdot(\overline{\bar{\tau}} \vec{V})
$$

with $H=E+p / \rho$ the total enthalpy.

The above Navier-Stokes equations are the vectorial formulation.

The tensor/index formulation of the Navier-Stokes equations is usually more practical and easy to use in theoretical analysis. It has the following form: -mass conservation equation:

$$
\frac{\partial \rho}{\partial t}+\frac{\partial}{\partial x_{i}}\left(\rho u_{i}\right)=0
$$

-momentum conservation equation:

$$
\frac{\partial\left(\rho u_{i}\right)}{\partial t}+\frac{\partial}{\partial x_{j}}\left[\rho u_{i} u_{j}\right]=\rho f_{i}+\frac{\partial}{\partial x_{j}}\left(\tau_{i j}-p \delta_{i j}\right) ; \quad i=1,2,3
$$

where the viscous stress tensor is:

$$
\tau_{i j}=\mu\left(\frac{\partial u_{i}}{\partial x_{j}}+\frac{\partial u_{j}}{\partial x_{i}}\right)-\frac{2}{3} \mu \frac{\partial u_{k}}{\partial x_{k}} \delta_{i j}
$$

$\delta_{i j}$ is the Kronecker delta, which is unity when $\mathrm{i}=\mathrm{j}$ and zero otherwise. -energy conservation equation:

$$
\frac{\partial}{\partial t}(\rho E)+\frac{\partial}{\partial x_{j}}\left[\rho u_{j} H\right]=\rho f_{j} u_{j}+q_{v}+\frac{\partial}{\partial x_{j}}\left(k \frac{\partial T}{\partial x_{j}}\right)+\frac{\partial}{\partial x_{j}}\left(\tau_{i j} u_{i}\right)
$$

In the following, the index formulation is used. Different forms of the NavierStokes equations are found in most text books on fluid dynamics, e.g. [18]. 


\section{Comments on Navier-Stokes equations}

From the above presented system of equations it is found that the fluid motion depends on many parameters. An essential role is attributed to ratio of the inertial and viscous forces, expressed in the Reynolds number. Three flow regimes can be distinguished based on this Reynolds number: laminar, turbulent and transitional flow. This concept is the basis of the boundary layer theory, to be discussed later in the turbulence modeling section.

The Navier-Stokes equations constitute a system of second-order non-linear partial differential equations. The non-linearity is mainly introduced by the inertial term $(\vec{V} \cdot \nabla) \vec{V}$, the term that with the viscous stress term govern turbulence.

The Navier-Stokes equations for a time-dependent flow describe the fluid motion in general and can be resolved most precisely by the direct numerical simulation (DNS) approach. However, the computational requirements for such kind of simulations at Reynolds numbers relevant for the present thesis are out of reach for the moment and some time to come (Reynolds number in the range of thousands can be considered, in close relation to the number of grid points). Simulations concerned with complex engineering problems within limited time frames are only possible when using reduced variants of the Navier-Stokes equations, like the Reynolds Averaged Navier-Stokes (RANS) as presented in the next section.

Note that, for the applications considered in the present thesis, the density of the liquid (water) is approximately constant (incompressible flow), while also the temperature is approximately constant, so that also the dynamic viscosity coefficient is constant. This assumption implies that from now the energy equation and the equations of state do not need to be addressed any more. This implies that the system refers only to the conservation equations for mass and momentum, involving $p$ and $\vec{V}$ as the unknown flow field quantities to be determined.

\section{Non-dimensional form}

Experimental studies of flows are often carried out on scaled models of true configurations. The results are displayed in dimensionless form, thus allowing scaling to full-scale conditions. The same approach can be undertaken in numerical studies as well. The governing equations can be transformed to dimensionless form by using appropriate normalizations. Velocities are normalized by a reference velocity $v_{0}$, spatial coordinates by a reference length $L_{0}$, time by a reference tine $t_{0}$ and pressure by $\rho v_{0}^{2}$. The dimensionless vari- 
ables are then:

$$
t^{*}=\frac{t}{t_{0}} ; \quad x_{i}^{*}=\frac{x_{i}}{L_{0}} ; \quad u_{i}^{*}=\frac{u_{i}}{v_{0}} ; \quad p_{i}^{*}=\frac{p}{\rho v_{0}^{2}}
$$

If the fluid properties $(\rho, \mu)$ are constant, the continuity equation in dimensionless form is:

$$
\frac{\partial u_{i}^{*}}{\partial x_{i}^{*}}=0
$$

When $f_{i}$ is the gravitational acceleration vector, the momentum equation becomes:

$$
S t \frac{\partial u_{i}^{*}}{\partial t^{*}}+\frac{\partial\left(u_{i}^{*} u_{j}^{*}\right)}{\partial x_{j}^{*}}=\frac{1}{R e} \frac{\partial^{2} u_{i}^{*}}{\partial x_{j}^{* 2}}-\frac{\partial p^{*}}{\partial x_{i}^{*}}+\frac{1}{F r^{2}} \gamma_{i}
$$

where $\gamma_{i}$ is the component in the $x_{i}$ direction of the normalized gravitational acceleration vector.

In equation 2.40 the dimensionless numbers are: the Strouhal number $(S t)$, the Reynolds number $(R e)$ and Froude number $(F r)$, defined as:

$$
\begin{gathered}
S t=\frac{L_{0}}{v_{0} t_{0}} \\
R e=\frac{\rho v_{0} L_{0}}{\mu} \\
F r=\frac{v_{0}}{\sqrt{L_{0} g}}
\end{gathered}
$$

\subsubsection{Reynolds Averaged Navier-Stokes model}

Often, in engineering, one is interested in knowing only a few quantitative properties of a turbulent flow, such as the time-averaged total forces and moments, the averaged engine inlet conditions and the averaged conditions in the ship propeller plane. These properties can be found by time-averaging the Navier-Stokes equations. Because it is based on ideas proposed by Osborne Reynolds over a century ago, the resulting equations are called the Reynolds-averaged Navier-Stokes equations.

When applying the Reynolds averaged approach to turbulence two effects 
arise. First, the unsteadiness is removed i.e. all unsteadiness is regarded as part of the turbulence. And second, the time averaging of the non-linear terms in the Navier-Stokes equations gives rise to new terms (turbulence Reynolds stresses and scalar fluxes) that must be modelled by so-called turbulence models.

To derive the Reynolds Averaged Navier Stokes (RANS) equations every flow field variable is considered as the sum of the time-averaged value $\langle a\rangle$ and the fluctuation $a^{\prime}$ as:

$$
a=\langle a\rangle+a^{\prime}
$$

Replacing each variable in the Navier-Stokes equations with this decomposition assuming a statistically steady flow, yields upon time-averaging, the equations for incompressible flow.

The continuity equation, becomes:

$$
\frac{\partial\left\langle u_{i}\right\rangle}{\partial x_{i}}=0
$$

The momentum equation becomes:

$$
\frac{\partial\left(\rho\left\langle u_{i}\right\rangle\right)}{\partial t}+\frac{\partial}{\partial x_{j}}\left[\rho\left\langle u_{i}\right\rangle\left\langle u_{j}\right\rangle\right]=-\frac{\partial\langle p\rangle}{\partial x_{i}}+\frac{\partial}{\partial x_{j}}\left(\left\langle\tau_{i j}\right\rangle-\rho\left\langle u_{i}^{\prime} u_{j}^{\prime}\right\rangle\right) ; \quad i=1,2,3
$$

In equation 2.46 the viscous stress tensor has the form:

$$
\left\langle\tau_{i j}\right\rangle=\mu\left[\left(\frac{\partial\left\langle u_{i}\right\rangle}{\partial x_{j}}+\frac{\partial\left\langle u_{j}\right\rangle}{\partial x_{i}}\right)-\frac{2}{3} \frac{\partial\left\langle u_{k}\right\rangle}{\partial x_{k}} \delta_{i j}\right]
$$

In the momentum equation 2.46 the term $\rho\left\langle u_{i}^{\prime} u_{j}^{\prime}\right\rangle$ represents the Reynolds stress tensor, which has 9 components from which because of symmetry 6 are distinct. Turbulence modelling concerns finding relations for these 6 components in terms of the time-averaged quantities. Note that the RANS equations are more complicated than the Navier-Stokes equations but the convergence of its solution is smoother and the solution is less complicated and requires less computational effort to obtain. Still, the time-averaged values are not representing a well-defined physical situation since an infinite number of different time variations give the same average. Also, in the momentum equations, extra terms that include cross-correlations of fluctuations appear, which add 6 unknown quantities to the system. To close the system of RANS equations one has to add extra equations for these turbulence variables.

More details into the treatment of turbulent flows can be found in [65], [82], [91], [9], [85], [33] and [28]. 


\subsubsection{Turbulence models}

The system of equations 2.45 and 2.46 needs extra relations for the components of the Reynolds stress tensor in order to be closed. This is achieved by introducing the concept of turbulence modelling.

Turbulence models are approximations for the Reynolds stress terms and none of them are valid for all types of flow. In general three types of approaches to turbulence modelling can be found: Eddy Viscosity models, Reynolds Stress models and Large Eddy Simulation models. Since all models are approximations and very much depend on the type of flow, choosing one of them requires knowledge of the nature of the flow. In the present thesis, due to the goal of developing a robust method to be used in industry, the eddy viscosity model is chosen and detailed in the following.

Eddy viscosity turbulence models are based on the Boussinesq hypothesis. In this approach the Reynolds stresses are related to the rate of the deformation tensor of the time-averaged velocity. The eddy viscosity models can be classified by the number of partial differential equations to be added to the time-averaged Navier-Stokes equations. They vary from zero equation (algebraic) models to 12 equation models. However, the most popular models are the two-equation models. Two equation models compute the turbulence kinetic energy and turbulence length scale for large eddy structures. As a rule, the first equation is the equation for the turbulent kinetic energy and the second one is the equation for the turbulent length scale or an equivalent variable.

\section{Boussinesq hypothesis}

In 1877 Boussinesq introduced a hypothesis to link the Reynolds stresses and turbulent scalar stresses to the local gradients of the mean flow field through a turbulent viscosity, expressed as:

$$
-\rho\left\langle u_{i}^{\prime} u_{j}^{\prime}\right\rangle=2 \mu_{t} S_{i j}-\frac{2}{3}\left(\mu_{t} \frac{\left\langle\partial u_{k}\right\rangle}{\partial x_{k}}+\rho k\right) \delta_{i j}
$$

In equation (2.48), $\mu_{t}$ is the turbulent eddy viscosity, $k$ is the turbulence kinetic energy:

$$
k=\frac{\left\langle u_{i}^{\prime} u_{i}^{\prime}\right\rangle}{2}
$$


and $S_{i j}$ is the mean rate of strain:

$$
S_{i j}=\frac{1}{2}\left(\frac{\left\langle\partial u_{i}\right\rangle}{\partial x_{j}}+\frac{\left\langle\partial u_{j}\right\rangle}{\partial x_{i}}\right)
$$

Turbulent viscosity is linked to turbulent kinetic energy $k$ and length $l$ via the Prandtl-Kolmogorov hypothesis:

$$
\mu_{t}=f_{\mu} C_{\mu}^{1 / 4} \rho k^{1 / 2} l
$$

In equation $2.51 f_{\mu}$ and $C_{\mu}$ are dimensionless numbers.

Furthermore, $\mu_{t}$ is related to the turbulence kinetic energy $k$ and dissipation rate $\varepsilon$ via:

$$
\mu_{t}=f_{\mu} \frac{C_{\mu} \rho k^{2}}{\varepsilon}
$$

Using equations 2.51 and $2.52, k, \varepsilon$ and $l$ are related by:

$$
\varepsilon=C_{\mu}^{3 / 4} \frac{k^{3 / 2}}{l}
$$

Turbulence models provide the value for the turbulent viscosity $\mu_{t}$. A turbulence model can be as simple as an algebraic relation from a constant value for $\mu_{t}$ to one partial differential equation for $k$ or two partial differential equations for $k$ and $\varepsilon / l /$ any other combination $k^{m} l^{n}$.

\section{Two-equation Eddy Viscosity models}

For engineering applications turbulence models with two transport equations are the most popular models. These models compute the turbulence kinetic energy $k$ and the turbulence length $l$ for large eddies. The length scale or an equivalent is computed from the second transport equation, while the first equation is for the turbulence kinetic energy. Starting from the NavierStokes equations the transport equation for the turbulence kinetic energy can be obtained. In a similar way the transport equation for the length scale is formulated. In the following, the equations for the turbulence models that have been used in the computations in the present thesis are presented. 


\section{Standard $\mathrm{k}-\varepsilon$ model}

The $\mathrm{k}-\varepsilon$ model is the most popular two-equation turbulence model. In this case the quantity $\varepsilon$ is the turbulence dissipation rate defined as: $k^{3 / 2} l^{-1}=\varepsilon$. From [17], the transport equation for the turbulence kinetic energy is:

$\frac{\partial}{\partial t}(\rho k)+\frac{\partial}{\partial x_{j}}\left[\rho u_{j} k-\left(\mu+\frac{\mu_{t}}{\sigma_{k}}\right) \frac{\partial k}{\partial x_{j}}\right]=\mu_{t}\left(P+P_{B}\right)-\rho \varepsilon-\frac{2}{3}\left(\mu_{t} \frac{\partial u_{i}}{\partial x_{i}}+\rho k\right) \frac{\partial u_{i}}{\partial x_{i}}+\mu_{t} P_{N L}$

where

$$
\begin{gathered}
P=S_{i j} \frac{\partial u_{i}}{\partial x_{i}} \\
P_{B}=\frac{g_{i}}{\sigma_{h, t}} \frac{1}{\rho} \frac{\partial p}{\partial x_{i}} \\
P_{N L}=-\frac{\rho}{\mu_{t}} \frac{\partial u_{i}^{\prime} u_{j}^{\prime}}{\partial x_{i}}-\left[P-\frac{2}{3}\left(\frac{\partial u_{i}}{\partial x_{i}}+\frac{\rho k}{\mu_{t}}\right) \frac{\partial u_{i}}{\partial x_{i}}\right]
\end{gathered}
$$

For linear models $P_{N L}$ is zero and $\sigma_{k}$ is the turbulent Prandtl number. The equation for the turbulence dissipation rate is:

$$
\begin{aligned}
\frac{\partial}{\partial t}(\rho \varepsilon) & +\frac{\partial}{\partial x_{j}}\left[\rho u_{j} \varepsilon-\left(\mu+\frac{\mu_{t}}{\sigma_{\varepsilon}}\right) \frac{\partial \varepsilon}{\partial x_{j}}\right]=C_{\varepsilon 1} \frac{\varepsilon}{k}\left[\mu_{t} P-\frac{2}{3}\left(\mu_{t} \frac{\partial u_{i}}{\partial x_{i}}+\rho k\right) \frac{\partial u_{i}}{\partial x_{i}}\right]+ \\
& +C_{\varepsilon 3} \frac{\varepsilon}{k} \mu_{t} P_{B}-C_{\varepsilon 2} \rho \frac{\varepsilon^{2}}{k}+C_{\varepsilon 4} \rho \varepsilon \frac{\partial u_{i}}{\partial x_{i}}+C_{\varepsilon 1} \frac{\varepsilon}{k} \mu_{t} P_{N L}
\end{aligned}
$$

Where $\sigma_{\varepsilon}$ is the turbulent Prandtl number. The coefficients are given in the table 2.1.

\begin{tabular}{|c|c|c|c|c|c|c|c|}
\hline$C_{\mu}$ & $\sigma_{k}$ & $\sigma_{\varepsilon}$ & $\sigma_{h, t}$ & $C_{\varepsilon 1}$ & $C_{\varepsilon 2}$ & $C_{\varepsilon 3}$ & $C_{\varepsilon 4}$ \\
\hline 0.09 & 1.0 & 1.22 & 0.9 & 1.44 & 1.92 & 1.44 & -0.33 \\
\hline
\end{tabular}

Table 2.1: Coefficients used in standard $k-\varepsilon$ turbulence model

The eddy viscosity $\mu_{t}$ is obtained via equation 2.51 with $f_{\mu}$ equal to unity. Then, via the Boussinesq hypothesis $\mu_{t}$ is introduced in the momentum conservation equation of the RANS equations.

\section{RNG k- $\varepsilon$ model}

The RNG $k-\varepsilon$ turbulence model also uses equation 2.54 for the turbulence kinetic energy $k$ and an extra term in the right-handed side of equation 2.58 
for the turbulence dissipation rate $\varepsilon$ :

$$
-\frac{C_{\mu} \eta^{3}\left(1-\eta / \eta_{0}\right)}{1+\beta \eta^{3}} \frac{\rho \varepsilon^{2}}{k}
$$

where $\eta=S \frac{k}{\epsilon}$ and $\eta_{0}$ and $\beta$ are empirical coefficients given in table 2.2.

The extra term, given in equation 2.59, represents the effect of mean flow distortion on the turbulence.

Also the coefficients have slightly different values as given in table 2.2.

\begin{tabular}{|c|c|c|c|c|c|c|c|c|c|}
\hline$C_{\mu}$ & $\sigma_{k}$ & $\sigma_{\varepsilon}$ & $\sigma_{h, t}$ & $C_{\varepsilon 1}$ & $C_{\varepsilon 2}$ & $C_{\varepsilon 3}$ & $C_{\varepsilon 4}$ & $\eta_{0}$ & $\beta$ \\
\hline 0.085 & 0.719 & 0.719 & 0.9 & 1.42 & 1.68 & 1.42 & -0.387 & 4.38 & 0.012 \\
\hline
\end{tabular}

Table 2.2: Coefficients used in RNG $k-\varepsilon$ turbulence model

The RNG k- $\varepsilon$ model has been used for the current simulations since it is thought to perform better for strongly swirling flows [17] than the standard $k-\varepsilon$ turbulence model.

\section{Modified RNG $k-\varepsilon$ model}

According to [24] and [86] a modification to the RNG $k-\varepsilon$ turbulence model is required when dealing with cavitating flows. To account for the effects of the compressibility of the liquid-vapor mixture on the turbulence structures, the turbulence viscosity needs to be modified.

In the turbulence viscosity, equation 2.51 , the density $\rho$ is replaced by a density function $f(\rho)$, so that:

$$
\mu_{t}=f_{\mu} \frac{C_{\mu} f(\rho) k^{2}}{\varepsilon}
$$

with $f(\rho)$ defined as:

$$
f(\rho)=\rho_{v}+\frac{\left(\rho-\rho_{v}\right)^{n}}{\left(\rho_{l}-\rho_{v}\right)^{n-1}}
$$

where $\rho_{l}$ and $\rho_{v}$ are the liquid and vapor density, respectively, and $n$ is a value varying from 7 to $15 . n=10$ is recommended by [24] and used successfully for different test cases. According to equation 2.61, $f(\rho)$ equals $\rho_{l}$ and $\rho_{v}$ in the liquid and vapor phase, respectively. The modification is only applied within the mixture layer, where vapor and liquid coexist.

In figure 2.1 the density function for different values of $n$ is shown. It is observed that the density $\rho$, when the liquid starts to cavitate is decreasing 


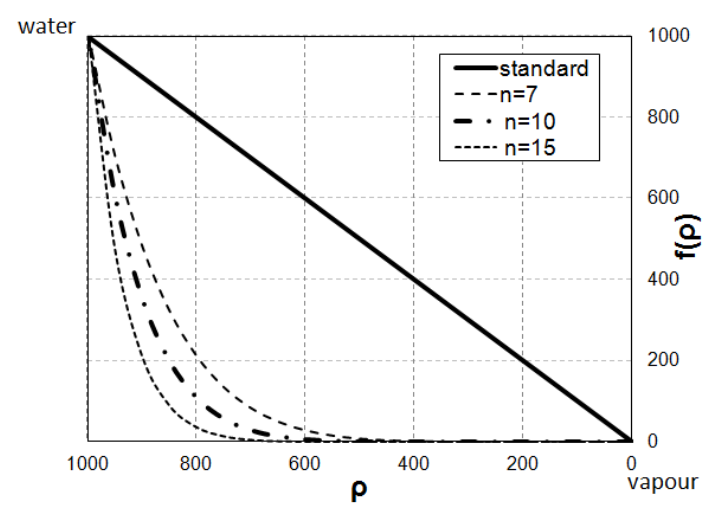

Figure 2.1: Variation density function as function of density

linearly, while with the modification the density drops rapidly in the beginning and then tends more slowly towards the vapor density.

This modification should result, for numerical simulations of cavitating flows, in more instabilities and in a stronger re-entrant jet in the case of sheet cavities. It therefore gives improved correlation with experimental results. The method was applied successfully in [24] and [86] to simulate unsteady flow structures.

In the 'Underling Flow Characteristics' chapter, results of the standard $k-\varepsilon$, the RNG $k-\varepsilon$ and the modified RNG $k-\varepsilon$ turbulence models are analysed for the case of the flow about a NACA0015 hydrofoil, as a benchmark test case. The most appropriate model in terms of agreement with the measurements will then be chosen.

\subsubsection{Wall functions}

In 1904, Ludwig Prandtl sets the basis of the boundary layer approach that divides the flow domain around a body in a very thin region close to the body surface where effects due to viscosity are important, called the inner layer and the remaining region where effects due to viscosity can be neglected, the outer layer. By this assumption Prandtl explained the effects of viscosity and reduces the complexity of the mathematical model.

Later in 1925, Prandtl introduced the mixing length concept, which allowed turbulent flows to be treated theoretically with the help of boundary layer theory. In this case the inner layer is divided in 3 sublayers: a linear sublayer, 
a logarithmic sublayer and a buffer/overlap sublayer.

In the linear sublayer the variations of the velocity is described by a linear function:

$$
u^{+}=y^{+}
$$

where $u^{+}$and $y^{+}$are a dimensionless velocity and a dimensionless normal distance to the wall, respectively.

In equation $2.62, u^{+}$and $y^{+}$are defined as:

$$
\begin{aligned}
u^{+} & =\frac{u}{u_{\tau}} \\
y^{+} & =\frac{u_{\tau} y}{\nu}
\end{aligned}
$$

where $u_{\tau}$, the friction velocity, is:

$$
u_{\tau}=\sqrt{\frac{\tau_{w}}{\rho}}
$$

and $\tau_{w}$ is the wall shear stress. Equation 2.62 is valid in the wall region within the range of $y^{+} \leqslant y_{m}^{+}$, which is given below.

In the logarithmic sublayer the variation of the velocity is described by a logarithmic function:

$$
u^{+}=\frac{1}{\kappa} \ln y^{+}+C, \text { for } y^{+}>y_{m}^{+}
$$

where $\kappa$ is the von Karman constant $\kappa=0.41$ and $C$ is a constant with a value around 5 (usually $C=5.25$ ).

The distance from the wall at which the linear sublayer transitions to the logarithmic sublayer is $y_{m}^{+}$and is defined by:

$$
y_{m}^{+}-\frac{1}{\kappa} \ln \left(E y_{m}^{+}\right)=0
$$

where $E$ is an empirical constant equal to 9.0 .

The third layer, the overlap sublayer, couples the linear sublayer with the logarithmic sublayer and is characterised by the interaction between the effects to due to molecular viscosity and effects due to turbulence. In the current approach the velocity distribution in the overlap layer is governed by the same equation as the linear sublayer since equation 2.62 is valid for $y^{+} \leqslant y_{m}^{+}$.

Equations 2.62 and 2.66 form the basis for the concept of wall functions. These functions prescribe the velocity distribution within the boundary layer 
and are valid for $y^{+}$values between 30 and 100, as recommended in [17]. In the study described in the present thesis, wall functions are employed. Details of other methods for modelling the parts of the boundary layer close to the wall are found in [18], [19] and [73].

\subsection{Cavitation Modeling}

\subsubsection{Introduction}

Multiphase flow terminology is referring to any macro flow regime (well above the molecular level) dealing with more than one phase or component of a fluid. In real life most of the mechanical parts within engines, waterjets, propellers and many other, deal with multiphase flows. Therefore the components working within multiphase flow regimes need to be designed accordingly. Modelling and predicting such flows is crucial for an optimum mechanical product.

When dealing with multi-phase flows, a distinction has to be made between two kinds of flows: segregated flows (a separated flow consists of separate, parallel streams of the two or more phases) and dispersed flows (a disperse flow pattern is one in which one phase or component is widely distributed as drops, bubbles, or particles in the other continuous phase), see [12]. This classification is not only important from a physical point of view but also from a modelling perspective, since for each category a different computational approach is required. For each type of multiphase flow two computational methods have been developed. For segregated multiphase flows, trajectory methods (Lagrangian) are used and for dispersed multiphase flows, two-phase methods (Eulerian) are available.

The Lagrangian methods (also named discrete particle methods or particle transport methods) are applicable for continuous-dispersed systems, in which the continuous phase is the fluid and the dispersed phase is formed by the particle / bubble / droplet.

Eulerian methods are applicable to continuous-dispersed systems for which two fluids models have been developed and also for continuous-continuous systems for which the homogenous flow model / volume of fluid (VOF) approach are available.

Based on above the description the multiphase models can be placed in a scheme as shown in figure 2.2.

The approach to numerically simulate such flows, as for any flow motion, starts from the equations of conservation of mass and momentum (RANS 
equations) which are solved in each of these methods (see figure 2.2). The presence of each phase is quantified by their volume fraction. The volume fraction is obtained by solving continuity equations for each phase / components with the constraint that the summation of the component volume fractions equals 1 .

The type of the flow to be analysed determines the approach to be taken for numerically simulating multiphase flows. In the present case, cavitation is a two-phase flow of two continuous fluids in which the main interest is in the large scale volume variations and not in the tracking of the small particles, therefore an Eulerian approach will be followed. Within this approach the most popular methods are: the level set method and the fraction of phase or VOF method. At present the level set method proves to be less used and less popular as mentioned by [36] compared to the VOF method. This appears to be primarily due to the lack of volume (mass) conservation and the higher computation cost. Therefore the chosen approach to be applied in the present thesis for numerical simulation of cavitating flow is the VOF method.

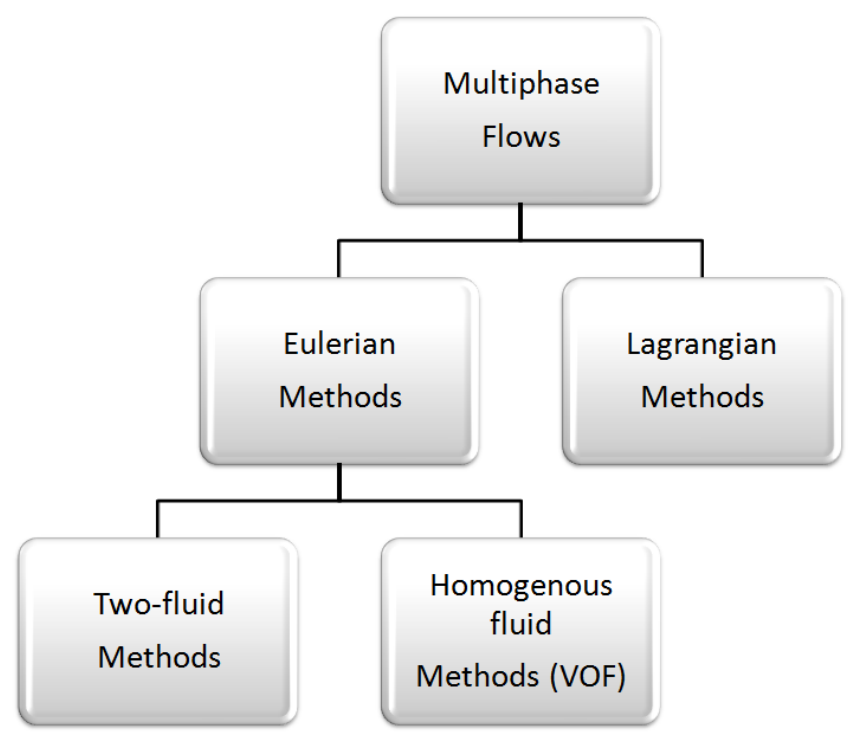

Figure 2.2: Scheme for classification of multi-phase methods

Two main issues need to be addressed when modeling cavitation: the treatment of the interface between the two phases and the transfer of mass from 
one phase to the other. Therefore the cavitation model is usually classified after on the approach taken for the mass transfer model and the production and the destruction of the vapor phase. However, the cavitation model is the sum of: the governing flow equations, the equations describing the cavitating flow (growth and collapse) which also gives the mass transfer model and the method for the capturing of the interface between the liquid and vapor phases, as schematically presented in figure 2.3. Each of these elements constituting the cavitation model are going presented and detailed below.

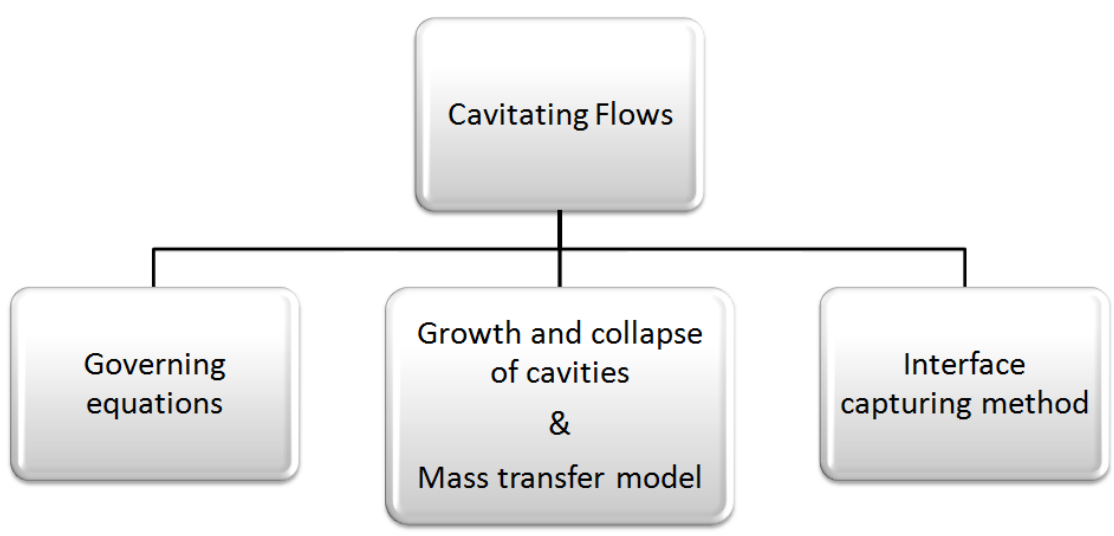

Figure 2.3: Scheme for cavitating flow

\subsubsection{Governing Equations}

The Navier-Stokes equations give the most general description of a fluid motion as previously described. Due to the complexity of a cavitating flow the equations solved are the time-averaged Navier-Stokes equations, namely the RANS equations. Therefore in the cavitating flow the continuity and momentum equations of the RANS equations are applied to each phase separately. It is also required to add an equation for the vapor mass transfer to the system of RANS equations to be applied to the cavitating flow. 


\subsubsection{Rayleigh-Plesset Cavity Source Terms}

The Rayleigh-Plesset equation describes the motion of a single spherical bubble in an infinite fluid domain subject to a variable pressure. Despite the complexity of the cavitating flow, already many basic results can be derived from the Rayleigh-Plesset equation.

Cavitation is often initiated from a micro bubble called nucleus, assumed spherical and typically a few to 100 microns in diameter. It is therefore crucial to understand and predict the evolution of such a bubble. The RayleighPlesset equation describes the evolution of a bubble in time due to a pressure change. The bubble is located within an infinite domain and is subjected to an input pressure $p_{\infty}(t)$.

The evolution in time of the bubble radius $R(t)$ satisfies the second-order ordinary differential equation:

$$
\rho_{l}\left[R \ddot{R}+\frac{3}{2} \dot{R}^{2}\right]=\left[p_{v}-p_{\infty}(t)\right]+p_{g_{0}}\left(\frac{R_{0}}{R}\right)^{3 \gamma}-\frac{2 S}{R}-4 \mu \frac{\dot{R}}{R}
$$

known as the Rayleigh-Plesset equation. This equation can be derived starting from the Navier-Stokes equations and is found in [11], [12], [10] and [30], among others.

In equation (2.68), $\dot{R}$ and $\ddot{R}$ are the first and second derivative with respect to time of the bubble radius and $R_{0}$ is the initial bubble radius. On the right hand side of equation 2.68 four terms are present:

(1) $\left[p_{v}-p_{\infty}(t)\right]$ is the driving term for bubble evolution, measuring the difference between the applied pressure $p_{\infty}(t)$ and the vapour pressure, inside the bubble.

(2) $p_{g_{0}}\left(\frac{R_{0}}{R}\right)^{3 \gamma}$ is the contribution of the non-condensable gas present inside the bubble.

(3) $\frac{2 S}{R}$ is the contribution in the balance equation of the surface tension $\mathrm{S}$. This term is only important for small radii.

(4) $4 \mu \frac{\dot{R}}{R}$ accounts for the effects of viscosity. Also, only important for small radii.

When neglecting the surface tension term $\frac{2 S}{R}$, the term due to the noncondensable gas $p_{g_{0}}\left({\frac{R_{0}}{R}}^{3 \gamma}\right)$ and the term due to viscous effects $4 \mu \frac{\dot{R}}{R}$, equation 2.68 becomes:

$$
\rho_{l}\left[R \ddot{R}+\frac{3}{2} \dot{R}^{2}\right]=p_{v}-p_{\infty}(t)
$$


Which is known as the Rayleigh equation.

Neglecting the second derivative in equation 2.69 results in:

$$
\dot{R}^{2}=\frac{2}{3} \frac{p_{v}-p_{\infty}}{\rho_{l}}
$$

Equation 2.70 describes the growth and collapse of a bubble in time and is the starting point of most of the cavitation models.

The most simplified model for bubble growth in time consequently becomes:

$$
\frac{d R}{d t}=\operatorname{sign}\left(p_{v}-p_{\infty}\right) \sqrt{\frac{2}{3} \frac{\left|p_{v}-p_{\infty}\right|}{\rho_{l}}}
$$

Clearly, equation 2.71 determines the bubble growth and bubble collapse based on the applied pressure $p_{\infty}$.

\subsubsection{Volume of Fluid method}

The volume of fluid is a method that captures the interface between vapor and liquid. It introduces the void fraction concept, defined as the ratio of the vapor volume $V_{v}$ and the total volume $V$ as:

$$
\alpha_{v}=\frac{V_{v}}{V}
$$

In equation 2.72 , the total volume $V$ within a cell is the sum of the volume of the components, the liquid volume $V_{l}$ and vapor volume $V_{v}$ :

$$
V=V_{v}+V_{l}
$$

If we consider the initial bubble volume $V_{0}=\frac{4}{3} \pi R_{0}^{3}$ and if $n_{0}$ is the number of initial bubbles per unit volume of the liquid, all equal in radius $R_{0}$, then the initial void fraction becomes:

$$
\alpha_{0}=\frac{V_{0} n_{0}}{1+V_{0} n_{0}}
$$

or

$$
\alpha_{0}=\frac{\left(\frac{4}{3} \pi R_{0}^{3}\right) n_{0}}{1+\left(\frac{4}{3} \pi R_{0}^{3}\right) n_{0}}
$$


Within the VOF approach, the fluid is treated as a single continuum with varying properties in space according to its composition $\alpha_{v}$. The void fraction $\alpha_{v}$ is derived from the solution of a transport equation for the component fluids.

The properties of the effective fluid vary in space according to the void fraction. The volume fraction of the components is determined from the condition:

$$
\alpha_{v}+\alpha_{l}=1
$$

While density and viscosity are defined as:

$$
\rho=\alpha_{l} \rho_{l}+\alpha_{v} \rho_{v}
$$

and

$$
\mu=\alpha_{l} \mu_{l}+\alpha_{v} \mu_{v}
$$

respectively.

The transport equations are the same for the effective fluid as in the case of a single phase flow, with the exception that density and viscosity vary sharply across the cavity surface of for example a sheet cavity.

\subsubsection{Mass transfer equation}

In the case of cavitation with the liquid and vapor fluid components present, the transport equation for vapor is similar to the mass conservation equation for liquid and is described by:

$$
\frac{\partial \alpha_{v}}{\partial t}+\nabla \cdot\left(\alpha_{v} \vec{u}\right)=S_{\alpha_{v}}
$$

In equation 2.79, $\vec{u}$ is the flow velocity, $S_{\alpha_{v}}$ represents the source of volume fraction of vapor. For this source term a number of cavitation models have been developed.

In order to close the system of equations formed by the RANS equations and the transport equation for the vapor, a cavitation model is introduced to specify the vapor production term $S_{\alpha_{v}}$. The vapor generation term is linked to the pressure field through the cavitation model.

The source term $S_{\alpha_{v}}$ is defined by the evolution of the bubbles with the 
initial volume fraction of vapor defined by the number of seed bubbles and their initial radius $R_{0}$ as in equation 2.75. Using Rayleigh's equation 2.71, equation 2.75 and the continuity equation for the effective fluid, the source term in equation 2.79 becomes:

$$
S_{\alpha_{v}}=\frac{4 \pi R^{2} n_{0}}{1+\left(\frac{4}{3} \pi R^{3}\right) n_{0}} \operatorname{sign}\left(p_{v}-p\right) \sqrt{\frac{2\left|p_{v}-p\right|}{\rho_{l}}}
$$

Different forms of terms have been defined starting from the Rayleigh-Plesset equation. The present source term, equation 2.80, is one of the simplest formulations and corresponds to Sauer's cavitation model, see [72]. The source term formula of bubble growth and collapse is known as the cavitation model, for details on other models like Schnerr and Sauer, Kuntz and Singhal see [75], [81], [44] and [78]. Different cavitation models are investigated in [31] and major differences in predicting cavitation are not found.

The cavitation modelling used throughout the present thesis is augmenting, the RANS governing equations together with the Rayleigh-Plesset source terms for cavitation and the VOF approach for interface tracking and mass transport. 


\section{Chapter 3}

\section{Vortex detection and visualization}

\subsection{Vortex definition}

Turbulent flow within engineering machineries is governed by the NavierStokes equations. When solving these equations by a numerical method, due to the large variety of length and time scales of the turbulence, one is often limited by the required computational effort. This has led to methods that are based on a mathematical model for turbulent flow simulations of reduced order of complexity.

Turbulent flows are dominated by coherent structures, the most common and known is the vortex, also referred to as an eddy. Vortices dominate the dynamics of the flow. Over the years many vortex definitions have been proposed, but with limited success. The definition of a vortex remains vague and therefore its prediction and detection likewise. One of the first and most general definitions was given by Lugt in 1972 [53]. It states that:

"A vortex is the rotation motion of a multitude of material particles around a common center."

A precise definition is difficult to formulate, a fact underlined by the numerous vortex detection methods available within literature; each trying to define a vortex structure and to use its definition in its detection. However, until today none of them is universally accepted or applicable.

A general more or less accepted definition of a vortex is that of a helical or swirling motion in a localized region, around a center. Note that, even though helicity $\left(H=\iiint \vec{u} \cdot \vec{\omega} d V\right)$ and vorticity $(\vec{\omega}=\vec{\nabla} \times \vec{u})$ are mathematical definitions, the vortex flow is not completely characterized by these 
quantities. More recently, driven by the need of having an accurate mathematical definition easy to implement employing results from CFD methods, Joeng and Hussain [38] have suggested that vortices must have two important properties: a) a vortex core must have a net vorticity and b) the geometry of the identified vortex core should be Galilean invariant (a detection method is Galilean invariant if the results do not change when a uniform velocity field is added to the existing velocity field).

Still, since there is no generally accepted vortex definition, its detection is still an open subject.

In the present Chapter insight is given in the most popular existing vortex detection methods and the method used in the present thesis.

\subsection{Vortex detection methods}

The increase in using CFD to analyse flow features, produced numerous methods for detection and visualization of vortical structures as underlined by Singer and Jiang in [8] and [40], respectively. The most popular vortex detection methods can be split in two categories: intuitive methods and mathematical methods, as detailed in following.

\subsubsection{Intuitive Methods}

\section{Vortex lines}

As already mentioned, one of the most intuitive parameter associated with a vortex detection method is vorticity, a vector quantity defined as the curl of the velocity by:

$$
\vec{\omega}=\vec{\nabla} \times \vec{v}
$$

where $\vec{v}$ is the velocity at a given point. In 1985/86, Moin and Kim [59] [60] use vortex lines (the integral curves of vorticity) to visualize vortical structures. A drawback of this method is that it is very sensitive to the choice of the starting point of the vortex lines. Thus, vortex lines as used for vortex detection can be misleading.

\section{Low pressure}

The second intuitive method is to use low pressure regions for vortex detection. Robinson et al. in 1989 [41] proposed that low pressure regions in incompressible turbulent flows almost always indicate vortex cores. Isosurfaces of low pressure are usually capable to capture the shape of a vortex 
core. The disadvantage of this method emerges when two vortices merge. Then the iso-pressure surfaces become indistinctive. Furthermore, a highquality image may require very fine grids.

\section{Spiral motion within the core}

In 1991 Robinson [71] formulated the following vortex definition and intuitive detection method:

"A vortex exists when instantaneous streamlines mapped onto a plane normal to the vortex core exhibit a roughly circular or spiral pattern, when viewed from a reference frame moving with the center of the vortex core."

The disadvantage of this method is that it requires the knowledge of the vortex core before identifying the vortex by the pattern of streamlines. Therefore, this simple method, based on the general definition of a vortex, can be considered as a visualization tool of other vortex detection methods.

\section{Maximum vorticity magnitude}

Zabusky et al. in 1991 [96], Villasenor and Vincent in 1992 [88], define the vortex core as the location of maximum vorticity magnitude. The difficulty of this method is that the regions with large vorticity magnitude do not necessarily correspond to vortices.

\section{Curvature and helicity}

Levy et al. 1990 [48], Yates and Chapman in 1991 [95] define the vortex core as an integral curve that has minimum curvature and contains the local maxima of the normalized helicity. The normalized helicity is a scalar quantity, defined everywhere except at the critical point, as:

$$
H_{n}=\frac{\vec{v} \cdot \vec{\omega}}{|\vec{v}||\vec{\omega}|}
$$

The assumption behind this method is that near vortices the angle between the velocity and the vorticity vector is small, i.e. that vorticity and velocity are co-parallel or anti-parallel. The method fails to follow the vortex core as stated by the authors.

\section{Vorticity-low pressure}

Banks and Singer in [8] propose a method to approximate the vortex skeleton based on the method of high vorticity magnitude and the method of low pressure. They consider locations where both the pressure is low and the 
magnitude of the vorticity is large in order to determine a vortex within a predictor-corrector algorithm. In contrast with the method of vortex lines, the method is self-correcting to the vortex core location through the use of the extra criterion on low pressure. The authors also suggest that any parameters for vortex core indication can be used. The disadvantage of the proposed method is that it uses two intuitive methods that both have issues predicting merging vortices when using coarse grids.

\subsubsection{Velocity Gradient Tensor Methods / Galilean In- variant Methods}

\section{Introduction}

In the fluid dynamics literature there are a number of methods for detecting/visualizing regions with concentrated vorticity. These methods consider the $3 \times 3$ tensor with the derivatives of the velocity vector. This is the socalled velocity gradient tensor $\overline{\bar{D}}=\vec{\nabla} \vec{v}$. The elements of this tensor give the rate at which an infinitesimal fluid element in the flow field deforms and rotates due to the gradient in the velocity field when moving with the flow field. The unit of these elements is $1 / \mathrm{s}$. The velocity gradient tensor can be expressed as:

$$
\begin{aligned}
\overline{\bar{D}}=\vec{\nabla} \vec{v} & =\left(\begin{array}{ccc}
\frac{\partial v}{\partial x} & \frac{\partial v}{\partial y} & \frac{\partial v}{\partial z} \\
\frac{\partial v}{\partial x} & \frac{\partial v}{\partial y} & \frac{\partial v}{\partial z} \\
\frac{\partial w}{\partial x} & \frac{\partial w}{\partial y} & \frac{\partial w}{\partial z}
\end{array}\right)=\frac{1}{2}\left(\begin{array}{cccc}
2 \frac{\partial v}{\partial x} & \frac{\partial v}{\partial y}+\frac{\partial v}{\partial x} & \frac{\partial v}{\partial z}+\frac{\partial w}{\partial x} \\
\frac{\partial v}{\partial y}+\frac{\partial v}{\partial x} & 2 \frac{\partial v}{\partial y} & \frac{\partial v}{\partial z}+\frac{\partial w}{\partial y} \\
\frac{\partial v}{\partial z}+\frac{\partial w}{\partial x} & \frac{\partial v}{\partial z}+\frac{\partial w}{\partial y} & 2 \frac{\partial w}{\partial z}
\end{array}\right)+ \\
& +\frac{1}{2}\left(\begin{array}{ccc}
0 & \frac{\partial v}{\partial y}-\frac{\partial v}{\partial x} & \frac{\partial v}{\partial z}-\frac{\partial w}{\partial x} \\
-\frac{\partial v}{\partial y}+\frac{\partial v}{\partial x} & 0 & \frac{\partial v}{\partial z}-\frac{\partial w}{\partial y} \\
-\frac{\partial v}{\partial z}+\frac{\partial w}{\partial x} & -\frac{\partial v}{\partial z}+\frac{\partial w}{\partial y} & 0
\end{array}\right)=\overline{\bar{S}}+\overline{\bar{\Omega}}
\end{aligned}
$$

Here the velocity gradient tensor $\overline{\bar{D}}$ has been split in the (symmetric) rate of strain tensor $\overline{\bar{S}}$, also called the rate of deformation tensor, and the (skewsymmetric) rate of rotation tensor $\overline{\bar{\Omega}}$.

The rate of strain tensor $\overline{\bar{S}}$ we express as: 


$$
\begin{aligned}
\overline{\bar{S}} & =\frac{1}{2}\left(\vec{\nabla} \vec{v}+(\vec{\nabla} \vec{v})^{T}\right)=\left(\begin{array}{ccc}
\frac{\partial v}{\partial x} & \frac{1}{2} \frac{\partial v}{\partial y}+\frac{\partial v}{\partial x} & \frac{1}{2} \frac{\partial v}{\partial z}+\frac{\partial w}{\partial x} \\
\frac{1}{2} \frac{\partial v}{\partial y}+\frac{\partial v}{\partial x} & \frac{\partial v}{\partial y} & \frac{1}{2} \frac{\partial v}{\partial z}+\frac{\partial w}{\partial y} \\
\frac{1}{2} \frac{\partial v}{\partial z}+\frac{\partial w}{\partial x} & \frac{1}{2} \frac{\partial v}{\partial z}+\frac{\partial w}{\partial y} & \frac{\partial w}{\partial z}
\end{array}\right)=\left(\begin{array}{ccc}
S_{11} & S_{12} & S_{13} \\
S_{12} & S_{22} & S_{23} \\
S_{13} & S_{23} & S_{33}
\end{array}\right)= \\
& =\left(\begin{array}{lll}
\vec{S}_{1} & \vec{S}_{2} & \vec{S}_{3}
\end{array}\right)
\end{aligned}
$$

with $\vec{S}_{1,2,3}$ the column (or row) vectors of tensor $\overline{\bar{S}}$.

Note that the trace of $\overline{\bar{S}}$ can be expressed as $\operatorname{trace}(\overline{\bar{S}})=\operatorname{trace}(\overline{\bar{D}})=\vec{\nabla} \cdot \vec{v}$, which will be zero for incompressible flow.

The Euclidean norm and determinat of rate of strain tensor $\overline{\bar{S}}$ are:

$$
\|\overline{\bar{S}}\|^{2}=S_{11}^{2}+S_{22}^{2}+S_{33}^{2}+2 S_{12}^{2}+2 S_{13}^{2}+2 S_{23}^{2}=\left|\overrightarrow{S_{1}}\right|^{2}+\left|\overrightarrow{S_{2}}\right|^{2}+\left|\overrightarrow{S_{2}}\right|^{2}
$$

and

$$
\operatorname{det}(\overline{\bar{S}})=\overrightarrow{S_{1}} \cdot\left(\overrightarrow{S_{2}} \times \overrightarrow{S_{3}}\right)
$$

, respectively.

It then also follows that for the velocity gradient tensor $\overline{\bar{D}}$ we find:

$$
\|\overline{\bar{D}}\|^{2}=\|\overline{\bar{S}}\|^{2}+\frac{1}{2}|\vec{\omega}|^{2}
$$

and

$$
\operatorname{det}(\overline{\bar{D}})=\operatorname{det}(\overline{\bar{S}})+\frac{1}{4}(\overline{\bar{S}} \vec{\omega}) \cdot \vec{\omega}
$$

, respectively.

In the analysis use is made of another tensor, the product of $\overline{\bar{S}}$ and $\overline{\bar{S}}$, which is given below in terms of the row (or column) vectors of the tensor $\overline{\bar{S}}$ as:

$$
\overline{\bar{S}} \overline{\bar{S}}=\left(\begin{array}{ccc}
\left|\overrightarrow{S_{1}}\right|^{2} & \overrightarrow{S_{1}} \overrightarrow{S_{2}} & \overrightarrow{S_{1}} \overrightarrow{S_{3}} \\
\overrightarrow{S_{1}} \overrightarrow{S_{2}} & \left|\overrightarrow{S_{2}}\right|^{2} & \overrightarrow{S_{2}} \overrightarrow{S_{3}} \\
\overrightarrow{S_{1}} \overrightarrow{S_{3}} & \overrightarrow{S_{2}} \overrightarrow{S_{3}} & \left|\overrightarrow{S_{3}}\right|^{2}
\end{array}\right)
$$

The corresponding trace can be expressed as trace $(\overline{\bar{S}} \overline{\bar{S}})=\|\overline{\bar{S}}\|^{2}$. The determinant of $\overline{\bar{S}} \overline{\bar{S}}$ can be expressed as $\operatorname{det}(\overline{\bar{S}} \overline{\bar{S}})=\left[\overrightarrow{S_{1}} \cdot\left(\overrightarrow{S_{2}} \times \overrightarrow{S_{3}}\right)\right]^{2}=$ $[\operatorname{det}(\overline{\bar{S}})]^{2}$.

The Euclidean norm of $\overline{\bar{S}} \overline{\bar{S}}$ can be expressed as: $\|\overline{\bar{S}} \overline{\bar{S}}\|^{2}=\|\overline{\bar{S}}\|^{4}-2\left\|\overline{\bar{S}}_{c}\right\|^{2}$ where $\overline{\bar{S}}_{c}$ is:

$$
\overline{\bar{S}}_{c}=\left(\begin{array}{lll}
\overrightarrow{S_{2}} \times \overrightarrow{S_{3}} & -\overrightarrow{S_{2}} \times \overrightarrow{S_{3}} \quad \overrightarrow{S_{1}} \times \overrightarrow{S_{2}}
\end{array}\right)
$$


which is a symmetric tensor. It follows that:

$$
\operatorname{trace}\left(\overline{\bar{S}}_{c}\right)=\frac{1}{2}(\vec{\nabla} \cdot \vec{v})^{2}-\frac{1}{2}\|\overline{\bar{S}}\|^{2}=\frac{1}{2}\left[(\operatorname{trace}(\overline{\bar{S}}))^{2}-\|\overline{\bar{S}}\|^{2}\right]
$$

The determinant of $\overline{\bar{S}}_{c}$ can be expressed as:

$$
\operatorname{det}\left(\overline{\bar{S}}_{c}\right)=\left[\overrightarrow{S_{1}} \cdot\left(\overrightarrow{S_{2}} \times \overrightarrow{S_{3}}\right)\right]^{2}=[\operatorname{det}(\overline{\bar{S}})]^{2}
$$

The Euclidean norm of $\overline{\bar{S}}_{c}$ can be expressed as:

$$
\left\|\overline{\bar{S}}_{c}\right\|^{2}=\left|\overrightarrow{S_{2}} \times \overrightarrow{S_{3}}\right|^{2}+\left|\overrightarrow{S_{1}} \times \overrightarrow{S_{3}}\right|^{2}+\left|\overrightarrow{S_{1}} \times \overrightarrow{S_{2}}\right|^{2}
$$

The elements of the rate of rotation tensor $\overline{\bar{\Omega}}$ are related to the components of the vorticity vector:

$$
\vec{\omega}=\vec{\nabla} \times \vec{v}=\left(\begin{array}{c}
\frac{\partial w}{\partial y}-\frac{\partial v}{\partial z} \\
\frac{\partial v}{\partial z}-\frac{\partial w}{\partial x} \\
\frac{\partial v}{\partial x}-\frac{\partial v}{\partial y}
\end{array}\right)=\left(\begin{array}{c}
\omega_{x} \\
\omega_{y} \\
\omega_{z}
\end{array}\right)
$$

so that

$$
\overline{\bar{\Omega}}=\frac{1}{2}\left(\vec{\nabla} \vec{v}-(\vec{\nabla} \vec{v})^{T}\right)=\frac{1}{2}\left(\begin{array}{ccc}
0 & -\omega_{z} & \omega_{y} \\
\omega_{z} & 0 & -\omega_{x} \\
-\omega_{y} & \omega_{x} & 0
\end{array}\right)
$$

The Euclidean norm of $\overline{\bar{\Omega}}$ equals $\|\overline{\bar{\Omega}}\|^{2}=\frac{1}{2}|\vec{\omega}|^{2}$.

The trace of $\overline{\bar{\Omega}}$ equals $\operatorname{trace}(\overline{\bar{\Omega}})=0$.

The determinant of $\overline{\bar{\Omega}}$ equals $\operatorname{det}(\overline{\bar{\Omega}})=0$.

Furthermore the product of $\overline{\bar{\Omega}}$ and $\overline{\bar{\Omega}}$ can be expressed as:

$$
\overline{\bar{\Omega}} \overline{\bar{\Omega}}=\frac{1}{4}\left(\begin{array}{ccc}
-|\vec{\omega}|^{2}+\omega_{x}^{2} & \omega_{x} \omega_{y} & \omega_{x} \omega_{z} \\
\omega_{x} \omega_{y} & -|\vec{\omega}|^{2}+\omega_{y}^{2} & \omega_{y} \omega_{y} \\
\omega_{x} \omega_{z} & \omega_{y} \omega_{z} & -|\vec{\omega}|^{2}+\omega_{z}^{2}
\end{array}\right)
$$

is a symmetric tensor with negative-valued diagonal elements.

Note that the trace of $\overline{\bar{\Omega}} \overline{\bar{\Omega}}$ can be expressed as: $\operatorname{trace}(\overline{\bar{\Omega}} \overline{\bar{\Omega}})=-\frac{1}{2}|\vec{\omega}|^{2}=$ $-\|\Omega\|^{2}$.

The determinant of $\overline{\bar{\Omega}} \overline{\bar{\Omega}}$ becomes $\operatorname{det}(\overline{\bar{\Omega}} \overline{\bar{\Omega}})=0$ and the Euclidean norm equals: $\|\overline{\bar{\Omega}} \overline{\bar{\Omega}}\|=\frac{1}{8}|\vec{\omega}|^{4}=\frac{1}{2}\|\Omega\|^{4}$.

The product of the rate of strain tensor $(\overline{\bar{S}})$ and the rate of rotation tensor 
$(\overline{\bar{\Omega}})$ can be expressed as:

$$
\overline{\bar{S}} \overline{\bar{\Omega}}=\frac{1}{2}\left(\begin{array}{lll}
\vec{S}_{1} \times \vec{\omega} & \vec{S}_{2} \times \vec{\omega} & \vec{S}_{3} \times \vec{\omega}
\end{array}\right)^{T}
$$

This tensor is not symetric in general.

The trace of $\overline{\bar{S}} \overline{\bar{\Omega}}$ is trace $(\overline{\bar{S}} \overline{\bar{\Omega}})=0$ and the Euclidean norm of $\overline{\bar{S}} \overline{\bar{\Omega}}$ equals: $\|\overline{\bar{S}} \overline{\bar{\Omega}}\|^{2}=\frac{1}{4}\|\overline{\bar{S}} \times \vec{\omega}\|^{2}$.

And the determinant of $\overline{\bar{S}} \overline{\bar{\Omega}}$ equals $\operatorname{det}(\overline{\bar{S}} \overline{\bar{\Omega}})=0$.

The velocity field satisfies the continuity equation (see also 2.31):

$$
\frac{\partial \rho}{\partial t}+\vec{\nabla} \cdot(\rho \vec{v})=0
$$

and the momentum equation, assuming the fluid to behave like a Newtonian fluid, as:

$$
\frac{\partial \vec{v}}{\partial t}+(\vec{v} \cdot \vec{\nabla}) \vec{v}=-\frac{1}{\rho} \vec{\nabla} p+\vec{f}+\frac{1}{\rho} \vec{\nabla} \cdot\left(2 \mu \overline{\bar{S}}+\mu_{2}(\vec{\nabla} \cdot \vec{v}) \overline{\bar{I}}\right)
$$

In the following it is assumed that the flow is incompressible ( $\rho=$ constant). This reduces the continuity equation to $\vec{\nabla} \cdot \vec{v}=0$, i.e. the velocity field is divergence free.

The momentum equation then reads, assuming the viscosity coefficients to be constant, in absence of external forces fields:

$$
\frac{\partial \vec{v}}{\partial t}+(\vec{v} \cdot \vec{\nabla}) \vec{v}=-\frac{1}{\rho} \vec{\nabla} p+\nu \nabla^{2} \vec{v}
$$

The governing equation for the derivatives of the velocity can be derived from equation 3.20. The result for the rate of strain tensor, is assuming constant viscosity $\mu$ :

$$
\frac{D \overline{\bar{S}}}{D t}+\overline{\bar{S}} \overline{\bar{S}}+\overline{\bar{\Omega}} \overline{\bar{\Omega}}=-\frac{1}{\rho} \overline{\bar{P}}+\nu \nabla^{2} \overline{\bar{S}}
$$

with $P_{i j}=\frac{\partial^{2} p}{\partial x_{i} x_{j}}$ the Hessian of the pressure field. Therefore $\overline{\bar{P}}$ is a symmetric tensor containing the second derivatives of $p(x, y, z, t)$ with respect to the spatial coordinates.

The trace of $\overline{\bar{S}}$ equals $\vec{\nabla} \cdot \vec{v}$, so then it follows from equation 3.21 , derived for incompressible flow, that:

$$
\frac{D(\nabla \vec{v} \vec{v})}{D t}+\|\overline{\bar{S}}\|^{2}+\|\overline{\bar{\Omega}}\|^{2}=-\frac{1}{\rho} \nabla^{2} p+\nu \nabla^{2}(\nabla \vec{\cdot} \vec{v})
$$


Therefore, in incompressible flow:

$$
\nabla^{2} p=\rho\left[\|\overline{\bar{\Omega}}\|^{2}-\|\overline{\bar{S}}\|^{2}\right]
$$

This is the Poisson equation for the pressure. Equation 3.23 is also valid for compressible flow in the case the viscosity coefficients are constant.

\section{Methods based on the analysis of the velocity gradient tensor}

The method considers the eigenvalues of $\overline{\bar{D}}$. It is hypothesized that, moving with the local velocity, the flow features closed or spiraling streamlines, a feature characteristic for compact regions with vorticity, if two of the three eigenvalues of $\overline{\bar{D}}$ are complex-valued. The eigenvalues follow from the characteristic polynomial associated with $\overline{\bar{D}}$ :

$$
\lambda^{3}+a_{1} \lambda^{2}+a_{2} \lambda+a_{3}=0
$$

where $a_{1}$ or $P, a_{2}$ or $Q$ and $a_{3}$ or $R$ are the invariants of $\overline{\bar{D}}$. The invariants have the following form:

$$
\begin{gathered}
a_{1}=-(\vec{\nabla} \cdot \vec{v})=-\operatorname{trace}(\overline{\bar{D}}) \\
a_{2}=\frac{1}{2}(\vec{\nabla} \cdot \vec{v})^{2}+\frac{1}{2}\left[\|\overline{\bar{\omega}}\|^{2}-\|\overline{\bar{S}}\|^{2}\right]
\end{gathered}
$$

and

$$
a_{3}=-\operatorname{det}(\overline{\bar{D}})
$$

Appendix A describes the procedure to determine the roots of the cubic polynomial. It requires the introduction of a number of quantities, i.e. $Q, R$, and $\Delta$ (see equation 4$)$ :

$$
\begin{gathered}
3 Q=a_{2}-\frac{1}{3} a_{1}^{2}=\frac{1}{2}\left[\frac{1}{2}|\vec{\omega}|+\frac{1}{3}(\vec{\nabla} \cdot \vec{v})^{2}-\|\overline{\bar{S}}\|^{2}\right]=\frac{1}{2}\left[\|\overline{\bar{\Omega}}\|^{2}-\|\overline{\bar{S}}\|^{2}+\frac{1}{3}(\vec{\nabla} \cdot \vec{v})\right] \quad(3.28) \\
2 R=-\left(\frac{2}{27} a_{1}^{3}-\frac{1}{3} a_{1} a_{2}+a_{3}\right)=-\frac{5}{54}(\vec{\nabla} \cdot \vec{v})^{3}-\frac{1}{6}(\vec{\nabla} \cdot \vec{v})\left(\frac{1}{2}|\vec{\omega}|-\|\overline{\bar{S}}\|^{2}\right)+\operatorname{det}(\overline{\bar{D}})
\end{gathered}
$$




$$
\Delta=Q^{3}+R^{2}
$$

In the remaining of the chapter we consider the case of incompressible flow $(\vec{\nabla} \cdot \vec{v}=0)$, thus:

$$
\lambda^{3}+a_{2} \lambda+a_{3}=0
$$

For which:

$$
\begin{gathered}
3 Q=\frac{1}{2}\left[\|\overline{\bar{\Omega}}\|^{2}-\|\overline{\bar{S}}\|^{2}\right] \\
2 R=\operatorname{det}(\overline{\bar{D}}) \\
\Delta=\frac{1}{216}\left[\frac{1}{2}|\vec{\omega}|^{2}-\|\overline{\bar{S}}\|^{2}\right]^{3}+\frac{1}{4}[\overline{\bar{D}}]^{2}=\frac{1}{216}\left[\|\overline{\bar{\Omega}}\|^{2}-\|\overline{\bar{S}}\|^{2}\right]^{3}+\frac{1}{4}[\operatorname{det}(\overline{\bar{D}})]^{2}
\end{gathered}
$$

The condition for two eigenvalues to be complex-valued is leading to the condition that the discriminant $\Delta=Q^{3}+R^{2}>0$. Three conditions for the vortex existence have been developed starting from the above relation for the velocity tensor invariants $R$ and $Q$. They are presented chronologically below.

1. The first method to use the invariants to determine a vortex structure is known as the Q-factor and was developed in 1988 by Hunt [92], in which the Q-factor is defined as in equation 3.28 with $\vec{\nabla} \cdot \vec{v}=0$. The vortex is then defined as the spatial region in which the Euclidean norm of the rate of rotation tensor dominates that of the rate of strain equivalent, so that: $Q=\frac{1}{6}\left[\|\overline{\bar{\Omega}}\|^{2}-\|\overline{\bar{S}}\|^{2}\right]>0$.

For incompressible flow the pressure satisfies equation 3.23, i.e. the Poisson equation $\nabla^{2} p=6 \rho Q$. If $Q>0$ the maximum of the pressure will be located on the boundary of the domain in which the Poisson equation is solved, so that the pressure wil have a minimum inside the domain. In practice $\mathrm{Q}$ is computed and it is determined whether the pressure is below some reference pressure.

According to [38] the Q-criterion does not represent the vortex geometry correctly for a number of investigated typical test cases.

2. Chong, Perry and Cantwell in 1990, Soria and Cantwell 1992 (see [20] and [21]) define the vortex core as the region in which the velocity gradient tensor has (two) complex-valued eigenvalues. This method is known as the $\Delta$-criterion, defined as:

$$
\Delta=\left(\frac{Q}{3}\right)^{3}+\left(\frac{R}{2}\right)^{2}=\frac{1}{216}\left[\|\overline{\bar{\Omega}}\|^{2}-\|\overline{\bar{S}}\|^{2}\right]^{3}+\frac{1}{4}[\operatorname{det}(\overline{\bar{D}})]^{2}>0
$$


In this case, the vortex is defined as a region in which the velocity gradient tensor admits two complex-valued eigenvalues: $\Delta>0$. In this region, the rate of rotation tensor does not need to dominate over the rate of strain tensor as long as the determinant of $\vec{\nabla} \vec{v}$ is large enough and $\Delta$ remains positive. This makes the $\Delta$-criterion less sensitive than the Q-criterion.

This method identifies most of the vortical structures in the flow, even the smallest structures, which may be a disadvantage.

\section{Method based on properties of the rate of strain tensor $\overline{\bar{S}}$}

The criterion considered next is based on the physical reasoning that in a vortex core the pressure tends to have a local minimum on the axis of the swirling flow field, such that the centrifugal force is balanced by the force due to the pressure gradient. The minimum in the pressure is sought in the plane perpendicular to the vortex axis. The pressure at a point can be expressed as $p(\vec{x}, t)=p\left(\overrightarrow{x_{0}}, t\right)+\left(\vec{x}-\overrightarrow{x_{0}}\right) \cdot \vec{\nabla} p+\frac{1}{2}\left(\vec{x}-\overrightarrow{x_{0}}\right)^{T} \overline{\bar{P}}\left(\vec{x}-\overrightarrow{x_{0}}\right)+\ldots$

At a minimum or maximum of the pressure $\vec{\nabla} p$ will be zero. In addition for the local pressure to be minimum in a plane at least two of the eigenvalues of the local pressure Hessian $\overline{\bar{P}}$ are positive. Since the Hessian is a symmetric tensor all its eigenvalues are real-valued. The Hessian follows from equation 3.21, i.e.:

$$
\overline{\bar{P}}=-\rho[\overline{\bar{S}} \overline{\bar{S}}+\overline{\bar{\Omega}} \overline{\bar{\Omega}}]+\mu \nabla^{2} \overline{\bar{S}}-\rho \frac{D \overline{\bar{S}}}{D t}
$$

In the criterion based on the eigenvalues of $\overline{\bar{P}}$ the effects of viscosity (decreasing the intensity of the vorticity field) and of the unsteady stretching are omitted. However, the relation between the actual pressure distribution and the modified pressure distribution that neglects the unsteady term and viscosity term, is not clear. Furthermore, for compressible flow extra terms have to be neglected related to the gradients in the density.

The so-called $\lambda_{2}$-criterion as formulated, by Jeong and Hussain in 1995 [38], is that at least two of the eigenvalues of $\overline{\bar{S}} \overline{\bar{S}}+\overline{\bar{\Omega}} \overline{\bar{\Omega}}$ (all real-valued because this tensor is symmetric) are negative. If the eigenvalues are numbered corresponding to their value, from negative to positive, the criterion is that the second eigenvalue of $\overline{\bar{S}} \overline{\bar{S}}+\overline{\bar{\Omega}} \overline{\bar{\Omega}}$ should be negative, i.e.

$$
\hat{\lambda}_{2}<0
$$


, which gives the criterion its name.

With:

$$
\overline{\bar{S}} \overline{\bar{S}}+\overline{\bar{\Omega}} \overline{\bar{\Omega}}=\left(\begin{array}{ccc}
\left|\overrightarrow{S_{1}}\right|^{2}+\frac{1}{4}\left(-|\vec{\omega}|^{2}+\omega_{x}^{2}\right) & \overrightarrow{S_{1}} \cdot \overrightarrow{S_{2}}+\frac{1}{4} \omega_{x} \omega_{y} & \overrightarrow{S_{1}} \cdot \overrightarrow{S_{3}}+\frac{1}{4} \omega_{x} \omega_{z} \\
\overrightarrow{S_{1}} \cdot \overrightarrow{S_{2}}+\frac{1}{4} \omega_{x} \omega_{y} & \left|\overrightarrow{S_{2}}\right|^{2}+\frac{1}{4}\left(-|\vec{\omega}|^{2}+\omega_{y}^{2}\right) & \overrightarrow{S_{2}} \cdot \overrightarrow{S_{3}}+\frac{1}{4} \omega_{y} \omega_{z} \\
\overrightarrow{S_{1}} \cdot \overrightarrow{S_{3}}+\frac{1}{4} \omega_{x} \omega_{z} & \overrightarrow{S_{2}} \cdot \overrightarrow{S_{3}}+\frac{1}{4} \omega_{y} \omega_{z} & \left|\overrightarrow{S_{3}}\right|^{2}+\frac{1}{4}\left(-|\vec{\omega}|^{2}+\omega_{z}^{2}\right)
\end{array}\right)
$$

Note that the trace of $\overline{\bar{S}} \overline{\bar{S}}+\overline{\bar{\Omega}} \overline{\bar{\Omega}}$ equals:

trace $[\overline{\bar{S}} \overline{\bar{S}}+\overline{\bar{\Omega}} \overline{\bar{\Omega}}]=\|\overline{\bar{S}}\|^{2}-\frac{1}{2}|\bar{\omega}|^{2}=\|\overline{\bar{S}}\|^{2}-\|\overline{\bar{\Omega}}\|^{2}$.

The Euclidean norm of $\overline{\bar{S}} \overline{\bar{S}}+\overline{\bar{\Omega}} \overline{\bar{\Omega}}$ is:

$\overline{\bar{S}} \overline{\bar{S}}+\overline{\bar{\Omega}} \overline{\bar{\Omega}}=\|\overline{\bar{S}} \overline{\bar{S}}\|^{2}-\|\overline{\bar{S}} \overline{\bar{S}}\|^{2}+\|\overline{\bar{\Omega}} \overline{\bar{\Omega}}\|^{2}$

And determinant of $\overline{\bar{S}} \overline{\bar{S}}+\overline{\bar{\Omega}} \overline{\bar{\Omega}}$ equals:

$\left.\operatorname{det}[\overline{\bar{S}} \overline{\bar{S}}+\overline{\bar{\Omega}} \overline{\bar{\Omega}}]=\left[\overrightarrow{S_{1}} \cdot\left(\overrightarrow{S_{2}} \times \overrightarrow{S_{3}}\right)\right]-\frac{1}{4}[\| \overline{\bar{S}}||]^{2}|\vec{\omega}|^{2}-\left|\overline{\overline{S_{C}}} \vec{\omega}\right|\right]+\frac{1}{16}|\overline{\bar{S}} \vec{\omega}|^{2}|\vec{\omega}|^{2}$

This method has difficulties to distinguish between vortices, see [8].

The characteristic polynomial $\operatorname{det}[\overline{\bar{S}} \overline{\bar{S}}+\overline{\bar{\Omega}} \overline{\bar{\Omega}}-\lambda \overline{\bar{I}}]=0$ of the tensor $\overline{\bar{S}} \overline{\bar{S}}+\overline{\bar{\Omega}} \overline{\bar{\Omega}}$ is expressed as:

$$
\lambda^{3}+b_{1} \lambda^{2}+b_{2} \lambda+b_{3}=0
$$

with, denoting the elements of $\overline{\bar{S}} \overline{\bar{S}}+\overline{\bar{\Omega}} \overline{\bar{\Omega}}$ as $b_{i j}$ :

$$
\begin{gathered}
b_{1}=-\left(b_{11}+b_{22}+b_{33}\right) \\
b_{2}=b_{11} b_{22}+b_{11} b_{33}+b_{22} b_{33}-b_{12}^{2}-b_{13}^{2}-b_{23}^{2} \\
b_{3}=-\left(b_{11} b_{22} b_{33}+2 b_{12} b_{13} b_{23}-b_{11} b_{23}^{2}-b_{22} b_{13}^{2}-b_{33} b_{12}^{2}\right)
\end{gathered}
$$

Deriving the coefficients in the characteristic equation we arrive to:

$$
\begin{gathered}
b_{1}=\|\overline{\bar{\Omega}}\|^{2}-\|\overline{\bar{S}}\|^{2} \\
b_{2}=\left\|\overline{\overline{S_{C}}}\right\|^{2}-\frac{1}{2}\|\overline{\bar{S}}\|^{2}\|\overline{\bar{\Omega}}\|^{2}-\frac{1}{4}\|\overline{\bar{S}} \vec{\omega}\|^{2}+\frac{1}{4}\|\overline{\bar{\Omega}}\|^{4} \\
b_{3}=-[\operatorname{det}(\overline{\bar{S}})]^{2}-\frac{1}{16}|\overline{\bar{S}} \vec{\omega}|^{2}|\vec{\omega}|^{2}+\frac{1}{4}\left\|\overline{\overline{S_{C}}}\right\|^{2}|\vec{\omega}|^{2}-\frac{1}{4}\left|\overline{\overline{S_{C}}} \vec{\omega}\right|^{2}
\end{gathered}
$$

Applying the same procedure as described in the Appendix to determine the roots of a cubic polynomial, it requires the introduction of a number of factors. The first one in $Q$, which here we denote as $\hat{Q}$. For a symmetric tensor 
this quantity is negative and it can be expressed as:

$$
3 \hat{Q}=b_{2}-\frac{1}{3} b_{1}^{2}
$$

Also:

$$
3 \hat{R}=-\left(\frac{2}{27} b_{1}^{3}-\frac{1}{3} b_{1} b_{2}+b_{3}\right)
$$

and

$$
\hat{\Delta}=\hat{Q}^{3}+\hat{R}^{2}
$$

, respectively.

In this case of real-valued eigenvalues $\hat{Q}<0$, though such that the discriminant $\hat{\Delta}=\hat{Q}^{3}+\hat{R}^{2}>0$. The eigenvalues are:

$$
\lambda_{1}+\frac{1}{3} b_{1}=2 \sqrt{-\hat{Q}} \cos \left(\frac{1}{3} \theta\right)
$$

with $\cos \theta=\hat{R} / \sqrt{-\hat{Q}^{3}}$

$$
\begin{aligned}
& \lambda_{2}+\frac{1}{3} b_{1}=2 \sqrt{-\hat{Q}} \cos \left(\frac{1}{3}(\theta+2 \pi)\right) \\
& \lambda_{3}+\frac{1}{3} b_{1}==2 \sqrt{-\hat{Q}} \cos \left(\frac{1}{3}(\theta+4 \pi)\right)
\end{aligned}
$$

\subsubsection{Discussion}

The vortex detection methods presented above are the most popular methods but, as already mentioned, they all have disadvantages. These methods do not predict accurately only vortices because they cannot distinguish between regions with $\mathrm{Q}$ and $\Delta$ falsely positive (distributed vorticity in e.g. mixing layers flow) and actual vortices. Typically all these methods have been used independently from each other but recent papers are combining several methods to improve the interactive visualization of vortex structures as in [79]. In the later reference, the vorticity and the $\lambda_{2}$-Galilean invariant criterion are used as a predictor-corrector method.

Since the vortex definition itself is not fool proof it is not surprising that none of the presented methods are adequate to capture vortex structures on their own. Therefore, for vortex detection at least two methods have to be combined: a mathematical method and an intuitive method. This kind of approach is sound and proves to be quite successful see [79]. This is also the method applied in the present thesis, as described later in this chapter. 


\subsection{Vortex visualization methods}

In close relation with the vortex detection methods, there are many vortex visualization procedures. A classification of the flow visualization approaches is presented in the following, starting with the most basic and proceeding to the most complex one as from [45].

\section{Direct flow visualization}

In this case of flow visualization the data are almost directly used as contour color plots and vectors, usually applied to the entire domain or to a large part of it. The visual aspect has a low level of abstraction and almost no conversion or extraction steps. This kind of direct visualization is difficult to be used in capturing the behaviour of the flow, such as vortices.

Texture-based visualization

Like a global technique, a texture is produced to generate a dense representation of the flow. They are mainly used to visualize the flow in $2 \mathrm{D}$ and on surfaces in 3D.

\section{Geometric visualization}

Geometry objects are features extracted from the data and used for visualization; examples are streamlines, iso-surfaces, particle trajectories etc. This kind of visualization provides a better representation of the behaviour associated with flow dynamics, and is therefore suitable to be used in vortex visualization.

\section{Feature-based visualization}

This is the most advanced technique of visualization and is basically extracting the physical meaning of patterns from the data set. The visualization is dependent on the data sets, the application and the research problem; it shows only the characteristic features of the flow, for example the vortical structures.

As in the case of the vortex detection methods, vortex visualization approaches are not entirely distinct. The best way of using them is case dependent and separate methods can be combined for understanding of analysed flow feature such as vortices.

\subsection{Discussion on the selected method}

In this section a detailed description of the applied method is given. The vortex detection method used to detect a vortex and to refine the visualization in the region identified are the main steps of the developed method.

Most of the presented methods have their roots in the prediction of tur- 
bulent boundary layers and shear flows. The goal of the present thesis is detection and visualisation of vortices present in the flow around propellers, using results from numerical simulations from solving the RANS equations with wall functions. Mainly free shear flow simulations are of interest within the present study. Note that, outside the boundary layer most of the proposed methods are fairly successful, especially, if as proposed, at least two methods are applied simultaneously.

In the following a description is given of the method, used in the thesis.

\subsubsection{Detection}

When dealing with vortices, the developed method is first used to detect the location of a vortex and subsequently the method is used to refine the grid in this vortical flow region.

Within the present thesis the method used to determine the location of the tip vortex is a predictor-corrector method that combines the intuitive method of searching for the local minimum pressure with the the Q-factor Galilean invariant method. The vortex prediction utilizes the Q-factor method and its location is then corrected with the minimum pressure method (also since cavitation is directly related to locations with low values of the pressure) by means of adding in the visualisation the cells in which the pressure is lower than a certain value. In figure 3.1 an example of a representative cell selection is shown.

\subsubsection{Grid optimization}

Two types of tip vortices are expected to be encountered in the test cases in the present study: a steady tip vortex above and downstream of the Elliptic 11 rake hydrofoil and a steady tip vortex developing near the propeller blade tips (the 4119DTRC propeller and the Leadind Edge skew propeller).

For every test case, the solution obtained on the initial "coarse" mesh is analysed first. Then the cells with a Q-factor higher than a certain value are selected and corrected using the low-pressure vortex method. This first range of cells gives a first indication of the location of the vortex, see figure 3.1. Once this selection has been made the selected cells are refined in all three directions and a new numerical simulation is carried out. This procedure is applied at most three times. Subsequently the number of cells selected is increasing rapidly and the produced meshes are becoming too large for the computer resources available. A cross flow view of a refined mesh is given in 


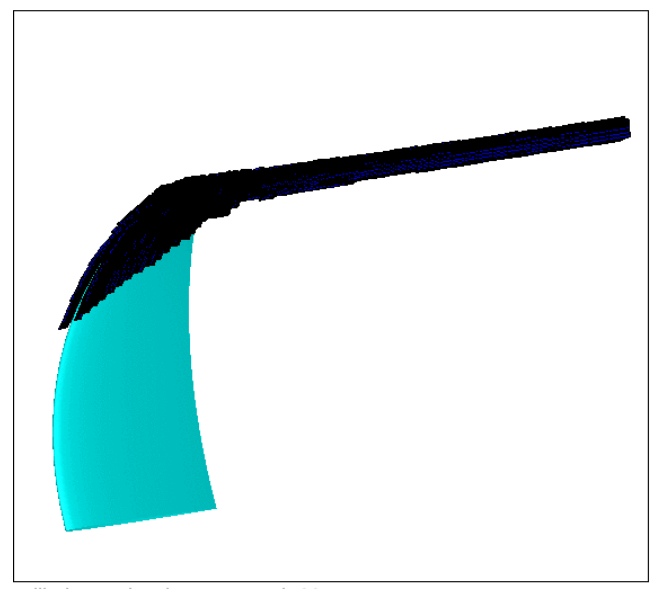

Figure 3.1: Cells to be refined after first simulation, initial mesh

figure 3.2 .

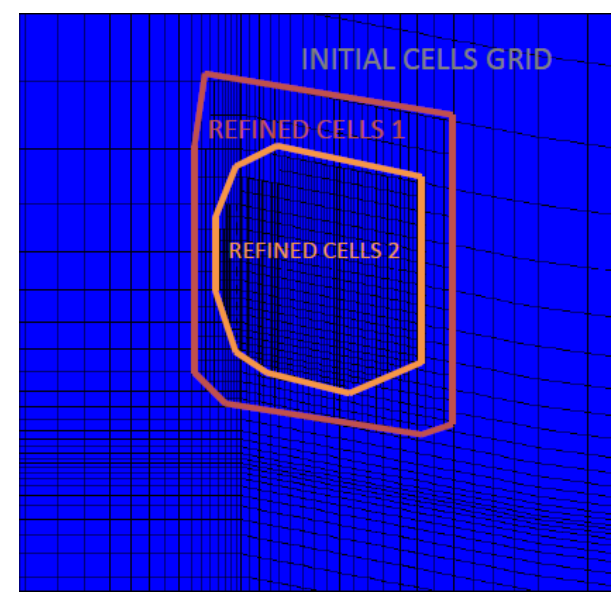

Figure 3.2: Cross-sectional view of grid refinement procedure

Figure 3.3 depicts the present procedure for tip vortex region refinement. In figure $3.3 Q 1>Q 2>Q 3 \ldots>Q n$ and $n$ usually equals 3. Note that the values of the $\mathrm{Q}$-factor selected for each refinement step are dependent on the case considered. In figure 3.3, $Q 1$ is the value used for identifying the first set 
of cells to be refined, and $Q 2$ and $Q 3$ are for the second and third selection of the cells to be refined. The relation between $Q_{1}, Q_{2}, \ldots$ is determined by the observation that for each refinement the region with positive $\mathrm{Q}$ factor within the tip vortex is decreasing, i.e. the vortex becomes compacter while the maximum value of $\mathrm{Q}$ is increasing, see figure 3.4. Thus for each new refinement in the core of the vortex the $\mathrm{Q}$ factor has to be increased.

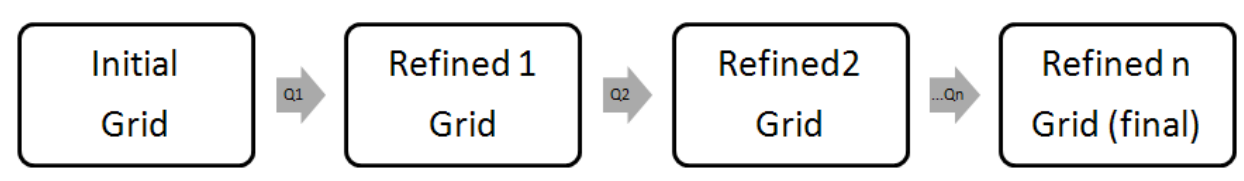

Figure 3.3: Q factor scheme

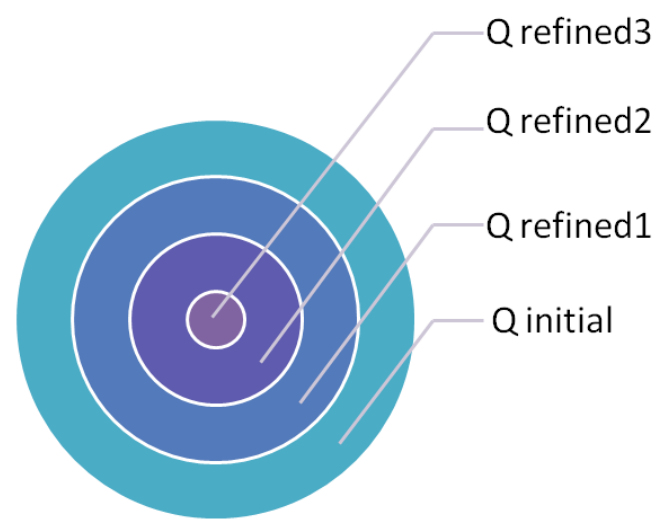

Figure 3.4: Variation of Q-factor iso-contour with mesh refinement

Therefore the scheme of figure 3.3 for tip vortex refinement can be described as follows:

(i) Numerical simulations are carried out for the initial grid for wetted flow conditions. 
(ii) The Q factor is computed for the converged solution. The cells with value of $\mathrm{Q} 2$ higher than a certain value $Q 1$ and a pressure coefficient below a certain value $C p 1$, determine the region in which the tip vortex resides.

(iii) The selected cells are refined in all three directions $\mathrm{x}, \mathrm{y}, \mathrm{z}$ and a second numerical solution is found which will feature a more compact tip vortex. This procedure is applied $n$ times and a final mesh for the region with the tip vortex is found.

This simple refinement method allows the user to obtain a well-defined tip vortex for geometries like wings and propellers.

\subsubsection{Visualization}

Using the above described vortex detection method, the visualization is based on computing the Q-factor, finding the cells with minimum pressure, using vapor concentration contours or velocity streamlines. The tip vortex visualization is based on a geometric visualization mainly employing iso-surface contours and particle trajectories. For each analysed case, the tip vortex refinement detection and visualization procedure will be described in detail.

\subsection{Conclusion}

For vortex detection within the computational domain, a number of methods have been proposed in the past but none of these methods can be considered as a stand-alone method. It is evident that, for the moment, one method for detecting vortical flow structures is not sufficient. Therefore a combination of two methods (one Galilean invariant and one intuitive method) in a predictor-corrector form is required. Moreover, the determined vortex has to be verified by an adequate visualization method like iso-contours and particle trajectories.

The current proposed method uses the Q-factor (since it is the easiest to implement) in the predictor role and corrects/verifies the results with the minimum pressure coefficient. The two step vortex detection method used for detection, grid refinement and visualization procedures will be applied to the following test cases: the Elliptic rake hydrofoil, the 4119 DTRC propeller and the Leading Edge Skew propeller. 


\section{Chapter 4}

\section{Assessment of uncertainty}

\subsection{Introduction}

The scope of the present chapter is to assess the uncertainty of the CFD method used, based on the ITTC - Recommended Procedures and Guidelines [4]. More investigations into CFD verification and validation can be found in [70] and [80].

The procedures for CFD verification and validation can be split in four steps: (1) preparation; (2) verification; (3) validation; and (4) documentation. The first step: preparation, involves the selection of the CFD method and the specification of the objectives, geometry, conditions, and the available benchmark information.

The second step: verification, is defined as a process for assessing the uncertainty of the results of the numerical simulation $\left(U_{S N}\right)$ and, when conditions permit, estimating the sign and magnitude of the numerical error $\left(\delta_{S N}^{*}\right)$ and corrected simulation numerical uncertainty $\left(U_{S_{C} N}\right)$. Sensitivity studies are conducted on effects of input parameters for the numerical procedure using a systematic variation of parameters, as described in the next section.

The third step: validation, is defined as a process for assessing uncertainty of the models implemented in the numerical method $\left(U_{S M}\right)$ by using benchmark experimental data and, when conditions permit, estimating the sign and magnitude of the simulation modelling error $\left(\delta_{S M}\right)$. The comparison error $(E)$ and validation uncertainty $\left(U_{V}\right)$ are defined in the next section.

The fourth step: documentation, is the detailed presentation of the CFD method (equations, initial and boundary conditions, modelling, and numerical methods), objectives, geometry, conditions, verification, validation, and analysis.

The first and last steps: preparation and documentation do not require much 
detail. The preparation step is test case dependent, therefore each test case within the thesis details this step. The description of the mathematical models is given in the Mathematical Modeling Chapter. In the following the verification method is detailed and the representative equations are defined for steady and unsteady flow. Guidelines related to the surface grid size and the time step selection result from the study on the sensitivity for the case of steady and unsteady flow, respectively.

Validation is carried out in the following chapters.

\subsection{Verification methodology}

Accuracy is defined as the closeness of agreement between a predicted value of a quantity and its true value. Error is the difference between a predicted value and the true value. Accuracy increases as the error approaches zero. The true value of the solution of the mathematical model is rarely known. Therefore the error must be estimated. An uncertainty $(U)$ is an estimate of the error such that the interval $\pm U$ contains the value of the error, 95 times out of 100. Therefore, an uncertainty interval indicates the range of likely magnitudes of the error, but no information about its sign.

The simulation error $\delta_{S}$ is defined as the difference between the result from a simulation $(S)$ and the true solution $(T)$ and is composed of additive modelling errors $\left(\delta_{S M}\right)$ and numerical errors $\left(\delta_{S N}\right)$. This leads to the error equation:

$$
\delta_{S}=S-T=\delta_{S M}+\delta_{S N}
$$

The uncertainty equation corresponding to the error equation is:

$$
U_{S}^{2}=U_{S M}^{2}+U_{S N}^{2}
$$

where $U_{S}$ is the uncertainty of the simulation, while $U_{S M}$ and $U_{S N}$ are the uncertainties of the model and numerical result, respectively.

For certain conditions, both the sign and magnitude of the numerical error can be estimated as $\delta_{S N}=\delta_{S N}^{*}+\varepsilon_{S N}$, with $\delta_{S N}^{*}$ an estimate of the sign and magnitude of $\delta_{S N}$ and $\varepsilon_{S N}$ the error in that estimate. The results of the numerical simulation is corrected to provide a numerical benchmark $S_{C}$, which is defined as:

$$
S_{C}=S-\delta_{S N}^{*}
$$


Verification is defined as a process for assessing the simulation numerical uncertainty $U_{S N}$ and, when conditions permit, estimating the sign and magnitude $\delta_{S N}^{*}$ of the numerical error of the numerical simulation and the uncertainty in that error estimate $U_{S_{C} N}$. For the uncorrected simulation approach, numerical error is decomposed into contributions from stopping the iterative solution method at some iteration number $\delta_{I}$, grid size $\delta_{G}$, time step $\delta_{T}$, and other parameters $\delta_{P}$. This gives the following expression for the uncertainty of the numerical simulation.

$$
U_{S N}^{2}=U_{I}^{2}+U_{G}^{2}+U_{T}^{2}+U_{P}^{2}
$$

For the corrected simulation approach, the solution is corrected to produce a numerical benchmark $S_{C}$ and the estimated numerical error of the result of the numerical simulation $\delta_{S N}^{*}$ is given by:

$$
\delta_{S N}^{*}=\delta_{I}^{*}+\delta_{G}^{*}+\delta_{T}^{*}+\delta_{P}^{*}
$$

Then the corrected uncertainty $U_{S_{C} N}$ follows from:

$$
U_{S_{C} N}^{2}=U_{I_{C}}^{2}+U_{G_{C}}^{2}+U_{T_{C}}^{2}+U_{P_{C}}^{2}
$$

\subsection{Verification CFD method: steady flow}

Results of verification and validation are presented for one single CFD method and for specified objectives, geometry, conditions, and available benchmark information. The CFD code is STAR-CD, which is a RANS method with a multitude of choices in difference schemes, turbulence models, and boundary conditions. The objective is to assess the verification process for the current CFD method for a benchmark test case of a steady state flow solution and for a test case for an unsteady condition.

\subsubsection{Geometry, Conditions, and Benchmark Data}

The Elliptic 11 Rake foil is a 3D wing with tip rake, specially designed to develop a strong cavitating leading edge and tip vortex. The profile of the foil is a NACA 0009 profile which is a symmetric profile without camber, with maximum thickness $t=0.09$. The upper surface is defined as:

$$
\frac{y}{c}=\frac{t}{0.20}\left(a_{0} \sqrt{\bar{x}}+a_{1} \bar{x}+a_{2} \bar{x}^{2}+a_{3} \bar{x}^{3}+a_{4} \bar{x}^{4}\right)
$$


Where $\bar{x}=\frac{x}{c} \in[0,1], \frac{y}{c} \in\left[0, t_{\max } / 2\right]$ and $a_{0}=0.269, a_{1}=-0.126, a_{2}=$ $-0.3516, a_{3}=0.2843$ and $a_{4}=-0.1015$. The chord distribution $C(y)$ is elliptical with a finite tip length $C_{T}$ :

$$
C(y)=\sqrt{C_{R}^{2}\left(1-y^{2}\right)+y^{2} C_{T}^{2}}
$$

for dimensionless span-wise coordinate $\mathrm{y}=[0,1], C_{T}=50 \mathrm{~mm}$ and $C_{R}=$ $150 \mathrm{~mm}$. The angle of attack distribution is defined by:

$$
\alpha(y, \beta)=\alpha_{t} y\left(\frac{e^{y_{\alpha} y}-1}{e^{y_{\alpha}}-1}\right)+\beta
$$

for $\mathrm{y}=[0,1], \alpha_{t}=11 \mathrm{deg}, \beta$ is the angle of attack for the entire geometry defined by the angle of attack of the root section and can vary from +3 to -3 degrees and $y_{\alpha}$ is a shape parameter set to 7 . Figure 5.16 shows the angle of attack distribution for $\beta=0$.

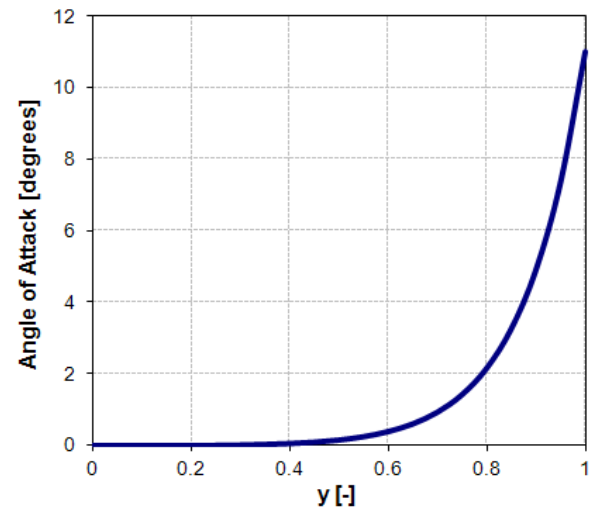

Figure 4.1: Angle of Attack, Elliptic 11 Rake foil

For the rake a similar distribution is used:

$$
R(y)=R_{t} y\left(\frac{e^{y_{R} y}-1}{e^{y_{R}}-1}\right)
$$

with $R_{t}=20 \mathrm{~mm}$ is the rake at the tip and the shape parameter $y_{R}=1$. For the above described geometry a steady inflow condition is considered for three grids. 


\subsubsection{Computational Grids}

The geometry used for the three-dimensional case is the Elliptic 11 Rake wing, with a NACA0009 profile, root chord of $0.15 \mathrm{~m}$ and tip chord of 0.05 $\mathrm{m}$ as described in [87].

The computational domain has: an inlet at 2 root chords upstream of leading edge, an outlet at 3 root chords downstream of the trailing edge. The foil has a section of 2 by 2 root chords, as in the experimental set-up, see figure 4.2 .

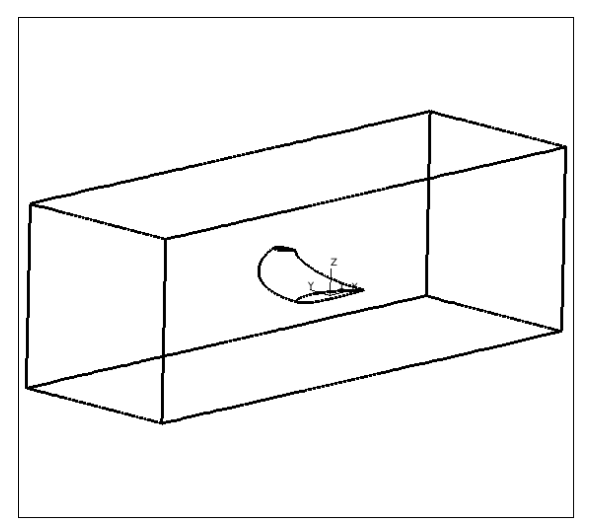

Figure 4.2: Elliptic 11 Rake foil computational domain

The mesh for the foil is created with a structured multi-block hexahedral mesh generator. The mesh near the blade is based on a O-type grid, to maintain control over the quality of the mesh near the blade. Development of the boundary layer along the blade surface is taken into account using wall functions. The requirements for the $\mathrm{y}^{+}$values on the foil surface can be met with an acceptable number of cells in the normal direction. Moreover, the aspect ratio and the differences in cell sizes can be kept low. Figure 4.2 shows the surface mesh of the Elliptic 11 Rake hydrofoil.

The O-grid around the profile is shown in figure 4.4. This type of meshing is efficient for control of $\mathrm{y}^{+}$, for which values of $\mathrm{y}^{+}$between 15 and 100, are as recommended for the approach using wall functions in [17].

At the upstream boundary an inlet boundary condition is applied, which requires the prescription of the velocity components and in addition values for the turbulence. Downstream an outlet boundary condition is applied with a constant pressure. This condition enables both inflow and outflow at the 
downstream boundary. At domain sides slip wall boundaries are applied. In figures 4.3 and 4.4, the foil surface mesh and the O-grid mesh are shown.

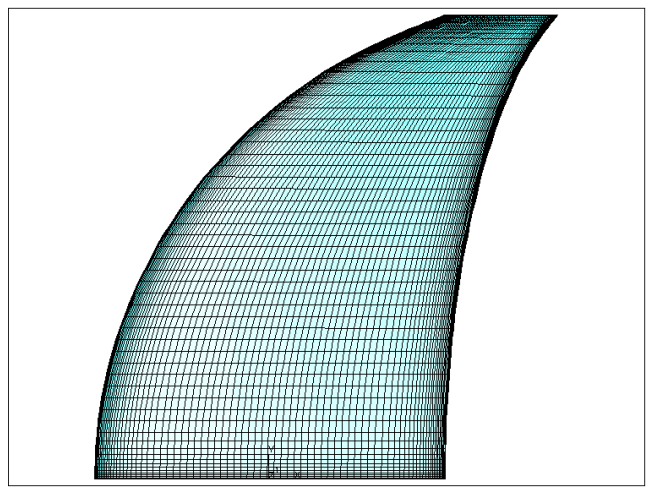

Figure 4.3: Surface mesh on Elliptic 11 Rake foil

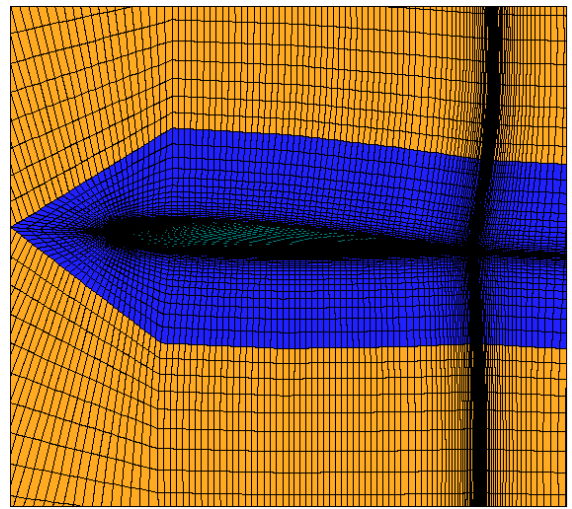

Figure 4.4: Structure of grid around the Elliptic 11 Rake foil 


\subsubsection{Verification of Integral Variables: Elliptic 11 Rake Hydrofoil}

The wetted flow over the Elliptic 11 Rake hydrofoil is computed for an inlet velocity of $7.43 \mathrm{~m} / \mathrm{s}$ and an outlet pressure of $21700 \mathrm{~Pa}$. Grid verification is assessed employing three meshes. Grids G2 and G3 are constructed based on an over-all mesh refinement of grid G3 with a grid ratio of $r_{G}=\sqrt{2}$, the grid details are given in table 4.1. The hydrofoil surface mesh for the three grids is presented in figures 4.5, 4.7 and 4.9. The value of $y^{+}$is kept at a value between 15 and 100 for all meshes, as required for the law of the wall, see [17].

Verification is performed considering the iterative procedure and grid convergence, i.e.

$$
\begin{gathered}
\delta_{S N}^{2}=\delta_{I}^{2}+\delta_{G}^{2} \\
U_{S N}^{2}=U_{I}^{2}+U_{G}^{2}
\end{gathered}
$$

The convergence of the iterative procedure has been assessed by examin-

\begin{tabular}{|c|c|c|c|}
\hline Grid & G3 & G2 & G1 \\
\hline Quality & Coarse & Medium & Fine \\
\hline Fluid Cells & 180320 & 494664 & 1261568 \\
\hline Contour Cells & 96 & 136 & 192 \\
\hline
\end{tabular}

Table 4.1: Grid characteristics for meshes used, Elliptic 11 Rake foil, angle of attack $\beta=3 \mathrm{deg}$

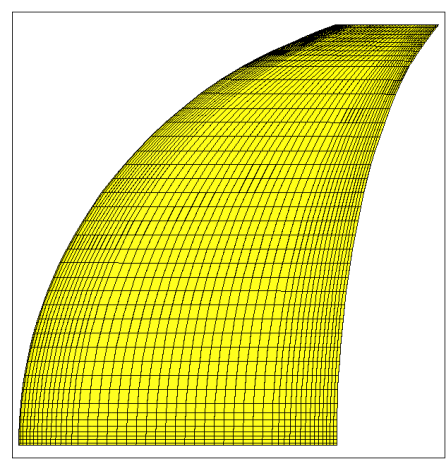

Figure 4.5: Surface mesh G3

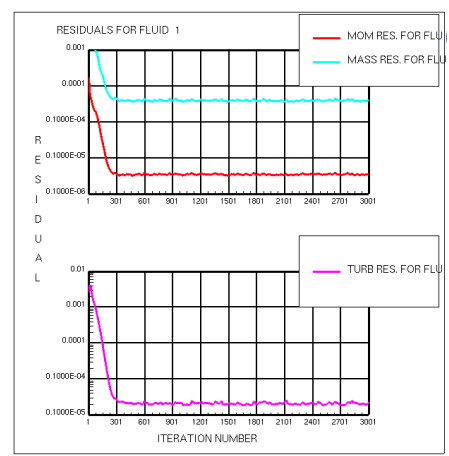

Figure 4.6: Residuals G3 


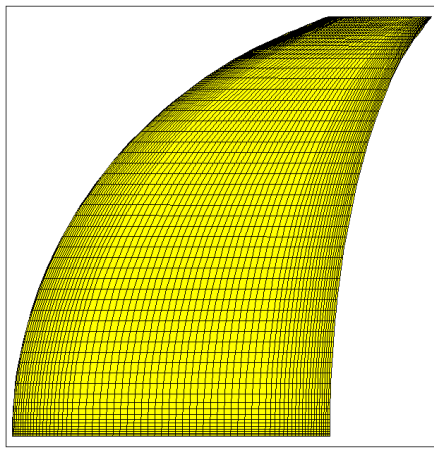

Figure 4.7: Surface mesh G2

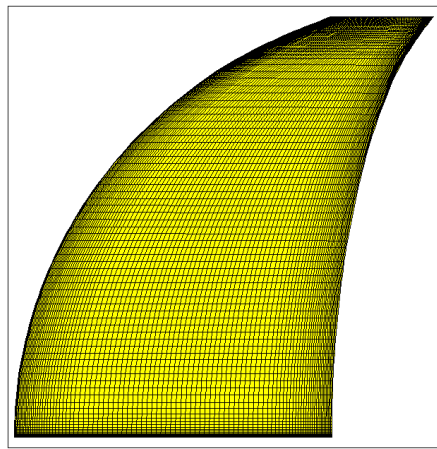

Figure 4.9: Surface mesh G1

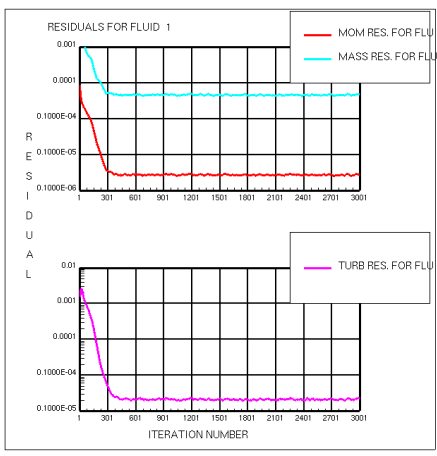

Figure 4.8: Residuals G2

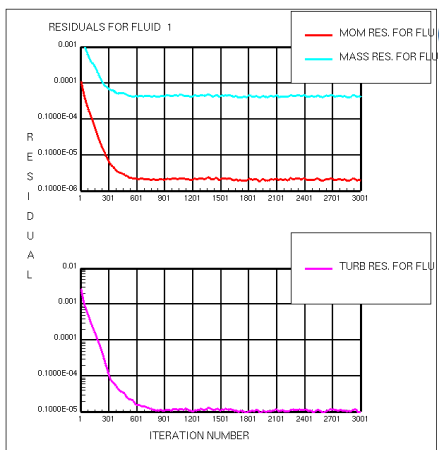

Figure 4.10: Residuals G1

ing the iteration history of the residuals of mass, momentum and turbulence equations, see figures 4.6, 4.8 and 4.10. For all three grids the iteration errors and uncertainties converge to values less than $+/-0.002 \%$. Thus, iteration errors and corresponding uncertainties are negligible in comparison to the grid errors and uncertainties for each of the three solutions as will be demonstrated in the following.

The results of lift and drag coefficients from the grid convergence study are summarized in tables 4.2 and 4.3, respectively. Here the lift and drag coefficients are defined as:

$$
C_{L}=\frac{L}{\frac{1}{2} \rho V^{2} c S}
$$




$$
C_{D}=\frac{D}{\frac{1}{2} \rho V^{2} c S}
$$

\begin{tabular}{|c|c|c|c|}
\hline Grid & G3 & G2 & G1 \\
\hline$C_{L}$ & 0.1294008 & 0.1276030 & 0.1266264 \\
\hline$\varepsilon$ & - & -0.0017978 & -0.0009742 \\
\hline
\end{tabular}

Table 4.2: Lift coefficient for the three grids employed

\begin{tabular}{|c|c|c|c|}
\hline Grid & G3 & G2 & G1 \\
\hline$C_{D}$ & 0.0097124 & 0.0088959 & 0.00872128 \\
\hline$\varepsilon$ & - & -0.00081650 & -0.00017445 \\
\hline
\end{tabular}

Table 4.3: Drag coefficient for the three grids employed

Using verification procedures guidelines provided by [4], differences between fine-medium grid $\left(\varepsilon_{12}\right)$ and medium-coarse grid $\left(\varepsilon_{23}\right)$ are used to define the convergence ratio $R$ and to determine the convergence condition. Solution differences between coarse $\left(S_{3}\right)$ to medium $\left(S_{2}\right)$ and medium to fine $\left(S_{1}\right)$ grids are defined by:

$\varepsilon_{21}=S_{2}-S_{1}$

$\varepsilon_{32}=S_{3}-S_{2}$

given in tables 4.2 and 4.3 for lift and drag coefficients, respectively.

For the ratio $R$ defined as $R=\varepsilon_{21} / \varepsilon_{32}$, three convergence conditions can be distinguished:

1. Monotonic convergence: When $0<R<1$, for every grid refinement the solution is changing a smaller amount towards the final values. This is the desired convergence behaviour.

2. Oscilatory convergence: When $R<0$, for every grid refinement the solution is oscillating around a final value.

3. Divergence: When $R>1$, for every grid refinement the variation in the solution is increasing, this indicates that the solution will diverge.

For the investigated condition detailed in table 4.4, the lift coefficient for the three grids (coarse G3, medium G2 and fine G1) and the corresponding solution differences between from medium to fine and coarse to medium 


\begin{tabular}{|c|c|}
\hline & Values \\
\hline Inlet velocity, $V$ & $7.43 \mathrm{~m} / \mathrm{s}$ \\
\hline Reynolds number, Re & $1.1 * 10^{6}$ \\
\hline Angle of attack, $\beta$ & $3 \mathrm{deg}$. \\
\hline
\end{tabular}

Table 4.4: Drag coefficient for the three grids employed

$\left(\varepsilon_{21}=S_{2}-S_{1}\right.$ and $\left.\varepsilon_{32}=S_{3}-S_{2}\right)$ are given in table 4.2 for which the ratio $R$ is $R=\varepsilon_{21} / \varepsilon_{32}=0.5418$.

Thus, based on previous classification, for the ratio $R$ between 0 and 1 the grid convergence is monotonic.

Next, following ITTC procedures [4], the generalized RE (Richardson Extrapolation) is used to estimate $U_{G}$ or $\delta_{G}^{*}$. Available methods are the Generalized Richardson Extrapolation and the GCI method see [4] and [80].

The generalized RE is used to estimate $U_{G}$ or $\delta_{G}^{*}$ and $U_{G_{C}}$.

The first-order RE estimate $\left(\delta_{R E_{G 1}}\right)$, the order of accuracy $\left(p_{G}\right)$ and the correction factor $\left(G_{G}\right)$ are:

$$
\begin{gathered}
\delta *_{R E_{G_{1}}}=\left(\frac{\varepsilon_{21_{G}}}{r_{G}^{p_{G}}-1}\right)=1.15 * 10^{-3} \\
p_{G}=\frac{\ln \left(\varepsilon_{32_{G}} / \varepsilon_{21_{G}}\right)}{\ln \left(r_{G}\right)}=1.7678 \\
C_{G}=\frac{r_{G}^{p_{G}}-1}{r_{G}^{p_{\text {est }}}-1}=0.845
\end{gathered}
$$

where $p_{G_{e s t}}=2$ is used in equation 4.17 .

For $C_{G}=0.845$ considered sufficiently close to 1 , the numerical error $\delta_{G}^{*}$ and simulation uncertainty $U_{G_{C}}$ are estimated (see [4]):

$$
\begin{gathered}
\delta_{G_{C}}^{*}=C_{G} \delta_{R E_{G_{1}}}^{*}=9.742 * 10^{-4} \\
U_{G_{C}}=\left(1-C_{G}\right) \delta_{R E_{G_{1}}}^{*}=1.7814 * 10^{-4}
\end{gathered}
$$

The corrected solution for the lift coefficient $S_{C}$ is defined by:

$$
S_{C}=S_{G_{1}}+\delta_{G_{1}}^{*}=0.1256
$$


thus $\delta_{G}^{*}$ and $U_{G_{C}}$ are $0.76 \%$ and $0.14 \% S_{C}$, respectively.

The level of verification is less than $0.8 \%$.

Applying the same equations we find that for the drag coefficient the corrected solution is: $S_{C}=0.0085470$. The results from the grid convergence study for the lift and drag coefficient $C_{L}$ and $C_{D}$ are summarized in tables 4.5 and 4.6. In table 4.6 the results are expressed as $\%$ of $S_{G 1}$.

\begin{tabular}{|c|c|c|c|}
\hline & $R_{G}$ & $p_{G}$ & $C_{G}$ \\
\hline$C_{L}$ & 0.54 & 1.76 & 0.84 \\
\hline$C_{D}$ & 0.21 & 4.45 & 3.68 \\
\hline
\end{tabular}

Table 4.5: Verification of Lift and Drag Coefficients

\begin{tabular}{|c|c|c|c|c|}
\hline & $U_{G}$ & $\delta_{G}^{*}$ & $U_{G_{C}}$ & $S_{C}$ \\
\hline$C_{L}$ & $0.91 \%$ & $0.76 \%$ & $0.14 \%$ & 0.1256546 \\
\hline$C_{D}$ & $3.45 \%$ & $2.00 \%$ & $1.45 \%$ & 0.0085470 \\
\hline
\end{tabular}

Table 4.6: Verification of Lift and Drag Coefficients 


\subsubsection{Verification of local variable: minimum pressure coefficient at mid-span}

As a local variable, the pressure coefficient is used to study for grid convergence. Iteration error and uncertainty are neglected in comparison with grid error and uncertainty. The variation of the minimum pressure on the suction side of the mid-span section of the Elliptic 11 rake hydrofoil (see figure 4.11) is investigated and investigated for grid error.

The pressure coefficient is defined as:

$$
C_{p}=\frac{P-P_{\infty}}{\frac{1}{2} \rho V_{\infty}^{2}}
$$

where $P$ is the local pressure, $P_{\infty}$ and $V_{\infty}$ is the far-field pressure and velocity, respectively and $\rho$ is the water density.

The variation of the minimum pressure coefficient at mid-chord of the Elliptic 11 Rake Foil with grid refinement is presented in table 4.7.

As for the previous uncertainty study of the global integral variables lift and drag coefficient, the same procedure is followed for the uncertainty assessment of the local variable, namely the minimum pressure coefficient. The ratio $\mathrm{R}$ of the solution changes equals 0.6804 ; which is in the desired range and is between 0 and 1 , indicating a monotonic convergence of the minimum pressure coefficient with grid refinement. The first-order RE estimate $\left(\delta_{R E_{G 1}}\right)$, order of accuracy $\left(p_{G}\right)$ and correction factor $\left(C_{G}\right)$ are estimated first, see table4.8, in order to determine the uncertainty and the error estimate.

Though $C_{G}=0.47$ is not close to 1 there might still be confidence (see [4]). Then the simulation numerical error $\delta_{G}^{*}=0.0144$ and simulation numerical uncertainty $U_{G_{C}}=0.0162$ are estimated at $1.84 \%$ and $1.08 \%$ of $S_{G 1}$, respectively. The grid corrected solution for the minimum pressure coefficient estimated with equation 2.3, equals $S_{C}=0.7939$.

\begin{tabular}{|c|c|c|c|}
\hline Grid & G3 & G2 & G1 \\
\hline$C_{p}$ & 0.7439 & 0.7651 & 0.7795 \\
\hline$\varepsilon$ & - & 0.0212 & 0.0144 \\
\hline
\end{tabular}

Table 4.7: Variation of minimum Pressure coefficient with grid refinement 


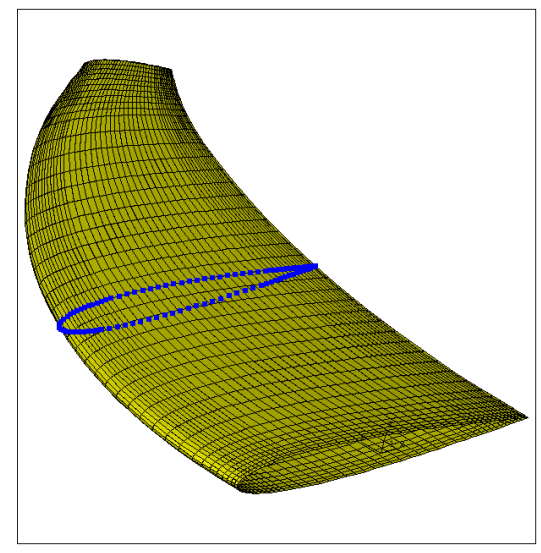

Figure 4.11: Mid-span section for surface grid G1, Elliptic 11 rake hydrofoil

\begin{tabular}{|c|c|c|c|}
\hline & $\delta_{R E_{G 1}}^{*}$ & $p_{G}$ & $C_{G}$ \\
\hline$C_{p}$ & $3.06 * 10^{-2}$ & 1.11 & 0.47 \\
\hline
\end{tabular}

Table 4.8: Verification parameters: minimum Pressure Coefficient

\subsubsection{Conclusions Steady Flow: Grid Guidelines}

Using the method from the uncertainty study provided by the ITTC, a verification of the current CFD method for the iterative procedure and mesh sensitivity has been performed.

Conclusions concerning meshing guidelines for 2D / 3D meshes to be used within the thesis can be drawn.

In table 4.9 a summary is presented of the three geometrically similar structured grids.

From table 4.9 the average area of the surface cells and the mesh density of the investigated grids can be found.

The averaged cell area is taken as the product of the averaged chord-wise extent and the averaged span-wise extent of the surface cells. In table 4.10 the averaged cell size is correlated to the difference, between the Richardson extrapolated value $\left(S_{C}\right)$ and the predicted value $\left(S_{G}\right)$.

Therefore $100 *\left(1-S_{C} / S_{G}\right)$. From the change of the solution, with decrease in the averaged area of the surface grid it is observed that: the lift coefficient on the coarse grid is within $2.9 \%$ from the corrected value, the drag coefficient 


\begin{tabular}{|c|c|c|c|}
\hline Parameter & G3(Coarse) & G2(Medium) & G1(Fine) \\
\hline Vertices & 4128 & 7888 & 16320 \\
\hline Vertices chord-wise & 96 & 136 & 192 \\
\hline Vertices span-wise & 43 & 58 & 86 \\
\hline Averaged chord-wise cell & $0.001 \mathrm{~m}$ & $0.0007 \mathrm{~m}$ & $0.0005 \mathrm{~m}$ \\
\hline Non-dimensional averaged chord-wise cell & 0.0104 & 0.0073 & 0.0052 \\
\hline Averaged span-wise averaged cell & $0.0047 \mathrm{~m}$ & $0.0034 \mathrm{~m}$ & $0.0024 \mathrm{~m}$ \\
\hline Non-dimensional averaged span-wise cell & 0.0232 & 0.0172 & 0.0117 \\
\hline
\end{tabular}

Table 4.9: Averaged cell characteristics of the surface grid of grids G1, G2 and G3, Elliptic 11 rake hydrofoil

\begin{tabular}{|c|c|c|c|}
\hline Parameter & G3(Coarse) & G2(Medium) & G1(Fine) \\
\hline Averaged cell area & 4.84 E-06 $m^{2}$ & $2.54 \mathrm{E}-06 \mathrm{~m}^{2}$ & $0.13 \mathrm{E}-06 \mathrm{~m}^{2}$ \\
\hline Non-dimensional averaged cell area & $24.2 \mathrm{E}-05$ & $12.6 \mathrm{E}-05$ & $6.12 \mathrm{E}-05$ \\
\hline Lift coefficient \% of $S_{C}$ & 2.90 & 1.53 & 0.77 \\
\hline Drag coefficient \% of $S_{C}$ & 12.00 & 3.92 & 2.00 \\
\hline Pressure coefficient \% of $S_{C}$ & -6.71 & -3.76 & -1.85 \\
\hline
\end{tabular}

Table 4.10: Elliptic 11 rake hydrofoil: deviation in \% of lift, drag and minimum pressure coefficient obtained from the corresponding corrected values $S_{C}$ for the the three grids used

within $12 \%$ and the minimum pressure coefficient is within $6.7 \%$. Therefore the accuracy of the drag coefficient determines the grid convergence requirement. At least an accuracy of $5 \%$ is desired for the value from numerical simulations, which is in line with the requirements on the accuracy of the measurements. Therefore the medium grid G2 is adequate for achieving the desired accuracy of about $1.5 \%$ in lift coefficient, $3.9 \%$ in drag coefficient and $3.7 \%$ in pressure coefficient of the estimated grid-independence values. This translates into a grid requirement of about $1.2 E-04$ non-dimensional averaged cell area on the surface of the hydrofoil. Table 4.9 then shows that, cells of about 0.007 non-dimensional chord-wise and 0.017 non-dimensional span-wise extent (in average 0.01) on the surface are desired for a grid that is adequate for numerical simulations within the thesis, see table 4.11.

However, this is a general guideline for a mesh. When complex flow structures like vortices and re-entrant jets appear more stringent requirements are likely. Also notice that meshes are in general not uniform and usually refined at locations like leading edge, trailing edge and tip of the hydrofoil or propeller, i.e. at location where a strong curvature in the stream-lines is expected. 


\begin{tabular}{|c|c|c|c|}
\hline Parameter & Chord-wise average cell & Span-wise & Average \\
\hline Non-dimensional average grid size & 0.007 & 0.017 & 0.01 \\
\hline
\end{tabular}

Table 4.11: Guideline average cell size for obtaining grid independent solutions, leading to an estimated grid error smaller then $5 \%$ variation of the drag coefficient

The above determined chord-wise and span-wise mesh dimensions together with an adequate value of $y^{+}$(corresponding to the law of the wall approach) form the grid guidelines that will be used as a minimum requirement for all surface meshes employed throughout this thesis. Whenever seemed necessary, the influence of the grid on the results needs to be analysed further.

\subsection{Verification CFD method: unsteady flow}

Within this thesis, the of cavitating flows are typically unsteady flows. Thus, the verification of a simple test case for unsteady flow (like the NACA 0015 section) is analysed in terms of steady (being the starting point of the unsteady flow) and unsteady flow. Emphasis is on turbulence modeling and on the effect of the time step on the flow solution. Since these parameters are not straightforward to verify as recommended by the ITTC rules, the ITTC procedure is not applied and a qualitative analysis of the obtained results is performed.

The main objective of this test case is to study the influence of turbulence and time step on the flow characteristics of a 2D shedding cavity: formation, re-entrant jet and cloud detachment, in a repetitive process.

A correct prediction of the behaviour of shedding cavitation is very important for predicting erosion due to the high pressures produced upon collapse of the bubble clouds. Note that also for the steady flow case a study of grid convergence and turbulence modeling is made. Tables (4.12 and 4.13) present a summary of the performed steady and unsteady flow simulations. Two grids have been used for the flow simulations employing the standard $\mathrm{k}$-epsilon turbulence model for wetted and cavitating flow.

The time step used in the computations is an important parameter of the unsteady flow simulation and the effect on the solution of the time step is studied by using three time steps: $1.0 \mathrm{E}-05 \mathrm{~s}, 1.0 \mathrm{E}-04 \mathrm{~s}$ and $1.0 \mathrm{E}-06 \mathrm{~s}$ for the RNG turbulence model.

For converging grid anf time step, turbulence modeling is investigated for the 
standard $k-\varepsilon$, the RNG $k-\varepsilon$ and the RNG $k-\varepsilon$ modified. In the latter model, the eddy viscosity is diminished near the vapor-water interface.

\begin{tabular}{|c|c|c|}
\hline Case & Grid & Turbulence model \\
\hline Steady (wetted) & G1 & $k-\varepsilon$ \\
\hline Steady (wetted) & G2 & $k-\varepsilon$ \\
\hline Steady (wetted) & G2 & RNG $k-\varepsilon$ \\
\hline
\end{tabular}

Table 4.12: NACA0015 steady flow simulations

\begin{tabular}{|c|c|c|c|}
\hline Case & Grid & Turbulence model & Time step \\
\hline Unsteady (cavitating) & G1 & $k-\varepsilon$ & $1.0 \mathrm{E}-05$ \\
\hline Unsteady (cavitating) & G2 & $k-\varepsilon$ & $1.0 \mathrm{E}-05$ \\
\hline Unsteady (cavitating) & G2 & RNG $k-\varepsilon$ & $1.0 \mathrm{E}-04$ \\
\hline Unsteady (cavitating) & G2 & RNG $k-\varepsilon$ & $1.0 \mathrm{E}-05$ \\
\hline Unsteady (cavitating) & G2 & RNG $k-\varepsilon$ & $1.0 \mathrm{E}-06$ \\
\hline Unsteady (cavitating) & G2 & RNG $k-\varepsilon$ modif. & $1.0 \mathrm{E}-05$ \\
\hline
\end{tabular}

Table 4.13: NACA0015 unsteady flow simulations

\subsubsection{Geometry: 2D NACA 0015 section}

The NACA0015 section is a widely used benchmark for 2D flow simulations and therefore numerous results are available, even though the test case is mainly used in numerical investigations, see [7] and [42].

The non-dimensional half thickness distribution of a four digit NACA section is:

$$
y=\frac{t}{0.20}\left(a_{0} \sqrt{\bar{x}}+a_{1} \bar{x}+a_{2} \bar{x}^{2}+a_{3} \bar{x}^{3}+a_{4} \bar{x}^{4}\right)
$$

Coefficients for the NACA0015 are presented in the table below, as from [5].

In the four digit NACA section (equation4.22) representation: $\bar{x} \in[0,1]$, $y \in\left[0, t_{\max } / 2\right], t$ is the thickness parameter, $\bar{x}=x / c$ is the dimensionless coordinate along the chord line ranging from 0 at the leading edge to 1 at the trailing edge, $c$ is the chord length and $a_{0}$ to $a_{4}$ are coefficients (given in 


\begin{tabular}{|c|c|}
\hline Parameter & Value \\
\hline $\mathrm{t}$ & 0.15 \\
\hline$a_{0}$ & 0.2969 \\
\hline$a_{1}$ & -0.126 \\
\hline$a_{2}$ & -0.3516 \\
\hline$a_{3}$ & 0.2843 \\
\hline$a_{4}$ & -0.1015 \\
\hline
\end{tabular}

Table 4.14: NACA0015 profile parameter values

table 4.14).

Within the current test case, the chord length of the profile is set to $c=$ $200 \mathrm{~mm}$ and the angle of attack is 6 degrees around the centre of gravity at $x / c=0.3086$, as recommended for the Virtue test cases of the EU project VIRTUE, see [37].

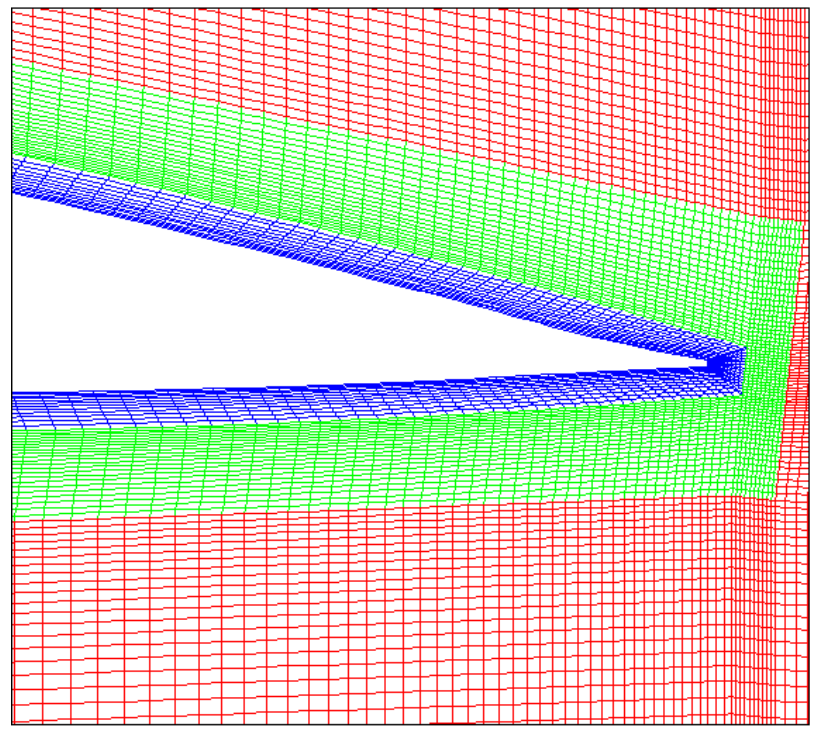

Figure 4.12: Visualisation of the O-grid around trailing edge of the NACA0015 section, G1 grid 


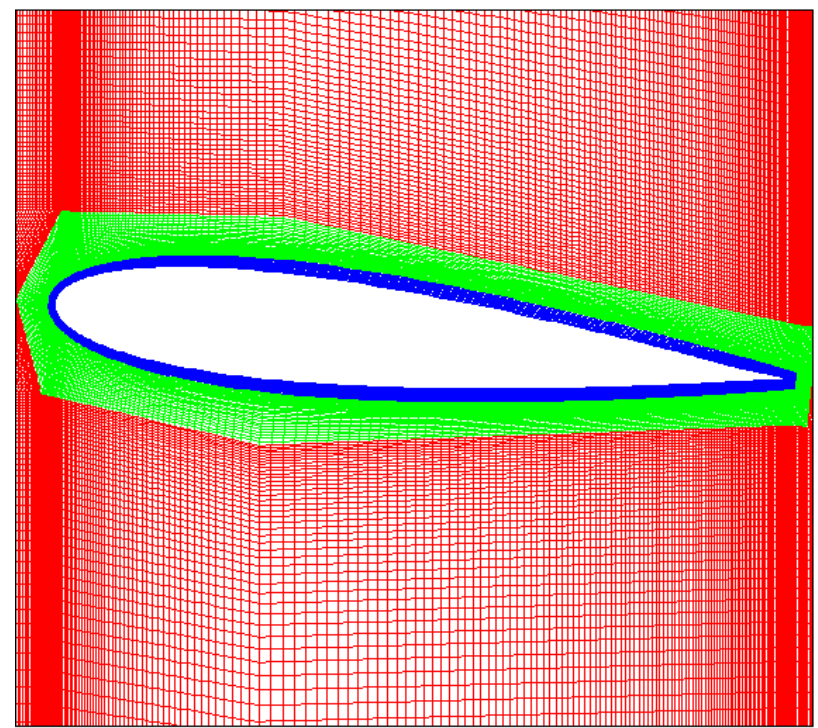

Figure 4.13: Visualisation of the O-grid around NACA0015 section, G1 grid

\subsubsection{Computational domain and mesh}

The domain size for the NACA0015 test case with chord length $c=200 \mathrm{~mm}$, is a rectangle of $1400 \times 570 \mathrm{~mm}^{2}$, with 2 chords extending upsteam of the LE to the inlet and 4 chords extending downsteam of the TE to the outlet. The mesh is a multi-block structured grid, with an O-grid type mesh around the section (including a blunt trailing edge, see figure 4.12). The O-mesh has been chosen in order to control the $\mathrm{y}^{+}$value at the surface, see figure 4.13. The applied boundary conditions are: an inlet velocity boundary type of 6 $\mathrm{m} / \mathrm{s}$, a turbulence intensity of $1 \%$, a pressure boundary type at the outlet and slip wall boundary conditions at the outer domain sides.

To assess the effect of mesh density on the results, two grid densities have been used. The results on the grids are analysed in the following. The coarse mesh G1 has 250 nodes around the profile and the finer mesh G2 has 418 nodes around the section. As concluded from the steady flow study, grid guidelines require about 0.007 non-dimensional grid size per averaged cell in chord-wise direction, to be sufficient to ensure grid convergence within +/$5 \%$ from the solution obtained through Richardson extrapolation. Thus the values of 0.008 and 0.0047 corresponding to grid G1 and grid G2, respectively, for the NACA0015 are in agreement with the grid guidelines, while 


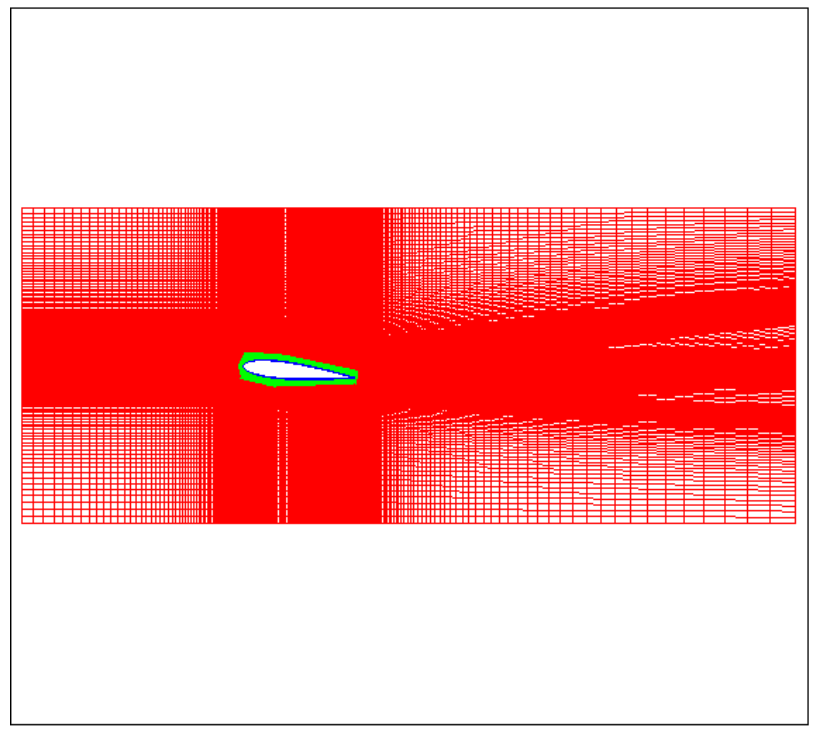

Figure 4.14: Visualisation of domain around NACA0015 section, G1 grid

grid G2 is well below the guidelines to ensure the assessed grid convergence.

\subsubsection{Settings of numerical parameters}

The present CFD simulations use an implicit SIMPLE algorithm to solve the RANS equations. The spatial discretization is second-order accurate in space using a MARS scheme (Monotone Advection and Reconstruction Scheme). A first order Euler Implicit scheme is used for the temporal dicretization. From all implemented spatial discretization schemes within the STAR-CD software, the MARS scheme has the least sensitivity to the mesh structure and skewness, see [17].

The turbulence models investigated for the present case are: the standard $k-\varepsilon$ model and the RNG $k-\varepsilon$ model for the finest mesh for the wetted flow conditions, namely. When cavitation is enabled a third turbulence model is employed: the modified RNG $k-\varepsilon$ turbulence model (described in the Mathematical Modeling Chapter).

The standard $k-\varepsilon$ model is used within the present study since it is one of the most popular models of the class of two-equation turbulence models. Its capability to predict cavitating flows needs to be assessed. The second 
choice, the RNG $k-\varepsilon$ turbulence model, is an improved $k-\varepsilon$ turbulence model recommended for cavitating swirling flows, see [17], [93] and [94]. The third choice, the modification of the turbulence viscosity, is also a popular choice when the RNG model fails to predict the behaviour of shedding cavities, see [24], [86] and [27].

The details for each turbulence model used have been presented in the Mathematical Modelling Chapter.

Moreover, the wall boundary condition used throughout the thesis employs the velocity law of the wall method since this does not require a large number of cells to be placed near wall. Its use does not affect the accuracy of the results of the analysed test case as shown through validation. The proper $y^{+}$ values (from 30 to 100 in average, as recommended in [17]) are pursued.

\subsubsection{Results wetted flow}

Results for wetted flow over the NACA0015 section at 6 degrees angle of attack $(\alpha), 6 \mathrm{~m} / \mathrm{s}$ inlet velocity corresponding to a Reynolds number $\mathrm{Re}=1197600$ are analysed first. Results have been obtained for various mesh densities and turbulence models (applied only for the finest mesh). Their influence on the performance of this section are assessed.

The convergence criteria based on [16] and [17] is set to $10^{-6}$ for the residual of the mass, momentum and turbulence equations. After 3000 iterations the numerical simulation is considered converged, as follows from figure 4.15, since the residuals do not appear to decrease to values below about $10^{-6}$. Also, the solution does not change more than about $0.001 \%$ (in wall forces). A representative distribution of the $y^{+}$values obtained for grid G2 is shown in Figure 4.16. The averaged values of $y^{+}$is around 20, except at the leading edge, where due to the low velocity at the stagnation point the value of $y^{+}$ drops towards a value of approximately 10. This is also the case near the trailing edge where the flow separates.

For this case the variation in mesh density and turbulence model (only for the finest mesh) is analysed to assess their influence on the section characteristics in terms of pressure, lift and drag coefficients.

The pressure coefficient is defined as:

$$
C_{p}=\frac{p-p_{0}}{\frac{1}{2} \rho_{\infty} U_{\infty}^{2}}
$$




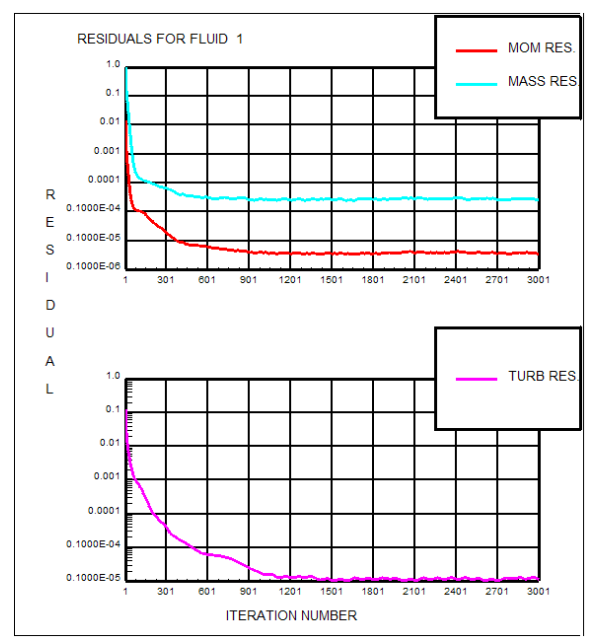

Figure 4.15: Residuals for equations for mass, momentum and turbulence, NACA0015 section at $\alpha=6$ degrees, $R e=1197600$, standard $k-\varepsilon$ turbulence model, in wetted flow, G2 grid

where $p_{0}=20334 \mathrm{~Pa}$ (corresponding to a cavitation number $\sigma=1$ in the cavitating flow case). The chord-wise distribution of pressure coefficient for grid G1 and grid G2, using the standard k- $\varepsilon$ turbulence model, and grid G2 using the RNG k- $\varepsilon$ model, are shown in figure 4.17 .

Figure 4.17 shows small differences between the three pressure distributions over the main part of the section, except at the leading edge, where more significant differences in results can be observed, primarily on the suction side.

Pressure coefficient contours for one of the analysed cases, G2 with the RNG $k-\varepsilon$ model are shown in figure 4.18.

\begin{tabular}{|c|c|c|c|c|}
\hline & Grid G1 $k-\varepsilon$ & Grid G2 $k-\varepsilon$ & Grid2 RNG & Analytical value \\
\hline$C_{p}$ stagnation & 1.08116 & 1.05329 & 1.01683 & 1.0 \\
\hline$C_{p}$ min & -1.86973 & -1.98888 & -2.06868 & - \\
\hline
\end{tabular}

Table 4.15: Pressure Coefficient at stagnation point and in suction peak, NACA0015 profile at $\alpha=6$ degrees, $R e=1.2 * 10^{6}$, wetted flow

Table 4.15 presents the values of the pressure coefficient at the stagnation 


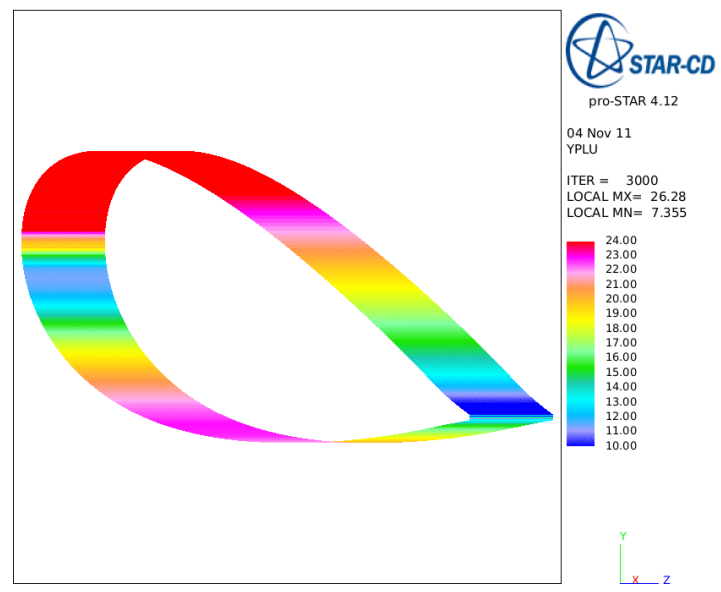

Figure 4.16: Distribution of $y^{+}$values, NACA0015 section at $\alpha=6$ degrees, $\mathrm{Re}=1.2^{*} 10^{6}$, wetted flow, $k-\varepsilon$ turbulence model, G2 mesh

point, as well as the minimum pressure in the suction peak on the upper surface.

From table 4.15 and figure 4.17 it is clear that the analysed cases over-predict the value of the stagnation pressure.

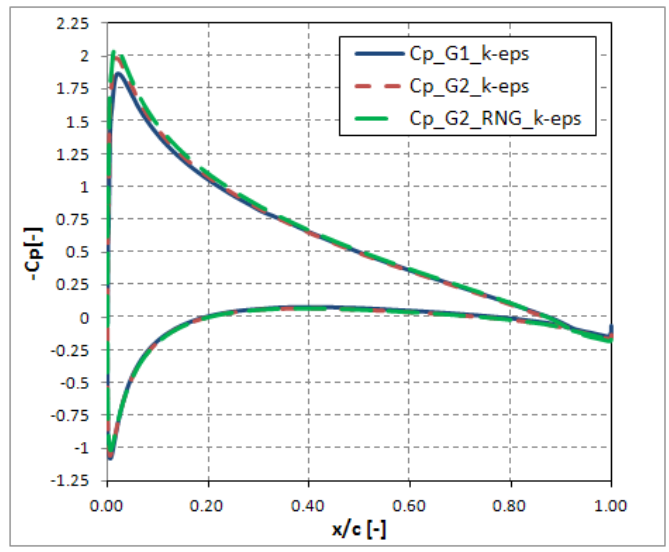

Figure 4.17: Distribution Pressure coefficient for different grids and turbulence models, NACA0015 profile at $\alpha=6$ degrees, $\mathrm{Re}=1.2^{*} 10^{6}$, wetted flow 


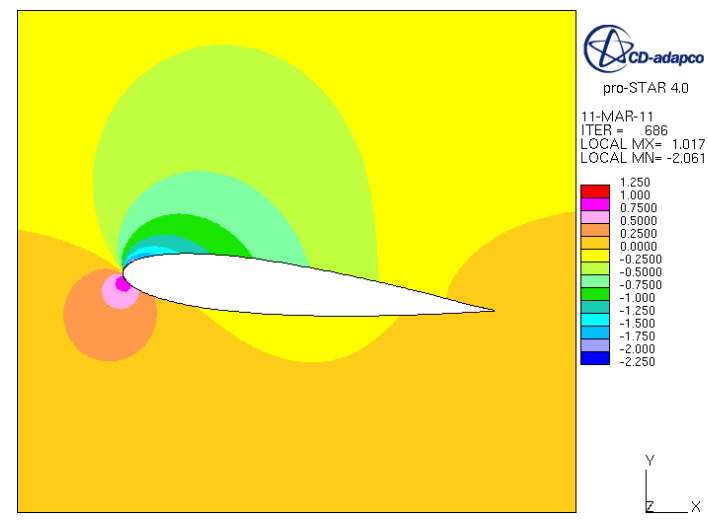

Figure 4.18: Pressure coefficient distribution obtained for NACA0015 section at $\alpha=6$ degrees, $\operatorname{Re}=1.2^{*} 10^{6}$, wetted flow, with RNG $k-\epsilon$ turbulence model, G2 grid

Table 4.15 shows that using the standard $k-\varepsilon$ turbulence model results in an over-prediction of the stagnation pressure with $8.1 \%$ and $5.3 \%$ for grid G1 and grid G2, respectively. The closest to the correct value of the stagnation pressure is produced with the RNG turbulence model: 1.01683 for grid G2, thus an $1.6 \%$ over-prediction. One explanation for the difference could be explained by the pressure at the inlet not being exactly the same as the prescribed pressure $\left(p_{0}=20334 P a\right)$ but this is often a problem introduced by turbulence models.

Over-prediction of the pressure coefficient in the stagnation point is a known issue of turbulence modelling which leads to an over-prediction of drag, see [13] and [61] for more details. Therefore, the best estimation of the stagnation pressure coefficient is obtained when employing the RNG turbulence model.

The minimum pressure shows substantial variation. The lowest value is obtained on the finest mesh for the RNG $k-\varepsilon$ turbulence model.

Other characteristics to be evaluated are the lift (l) and drag (d) of the section, expressed as non-dimensional coefficients:

$$
c_{l}=\frac{l}{\frac{1}{2} \rho_{\infty} U_{\infty}^{2} c}
$$




$$
c_{d}=\frac{d}{\frac{1}{2} \rho_{\infty} U_{\infty}^{2} c}
$$

Table 4.16 shows the lift and drag coefficients for the NACA 0015 section for different grid densities and turbulence models. The predicted lift coefficient is in the same range for all analysed cases (variations within 5\%), while only the drag coefficients predicted with the RANS method employing the RNG turbulence model are in good agreement with measured values. Variations of about $30 \%$ in $c_{d}$ occur when switching to a different grid or turbulence model.

\begin{tabular}{|c|c|c|c|c|}
\hline & Grid G1 $k-\varepsilon$ & Grid G2 $k-\varepsilon$ & Grid G2 RNG & EXP \\
\hline$c_{l}$ & 0.6312 & 0.6479 & 0.6671 & 0.658 \\
\hline$c_{d}$ & 0.0290 & 0.0192 & 0.0137 & 0.014 \\
\hline
\end{tabular}

Table 4.16: Lift and Drag Coefficients for NACA0015 at $\alpha=6$ degrees, $R e=$ $1.2 * 10^{6}$, wetted flow. Experimental data from [37]

Lift and drag coefficients decomposed in pressure and friction components are presented in tables 4.17 and 4.18 .

\begin{tabular}{|c|c|c|c|}
\hline & Grid G1 $k-\varepsilon$ & Grid G2 $k-\varepsilon$ & Grid2 RNG \\
\hline$c_{l}$ & 0.631235 & 0.647907 & 0.667112 \\
\hline Pressure $c_{l}$ & 0.61301 & 0.647912 & 0.667122 \\
\hline Friction $c_{l}$ & -0.000066 & -0.000005 & -0.00001 \\
\hline
\end{tabular}

Table 4.17: Components lift coefficients for NACA0015 section at $\alpha=6$ degrees, $\operatorname{Re}=1.2^{*} 10^{6}$, wetted flow

\begin{tabular}{|c|c|c|c|}
\hline & Grid G1 $k-\varepsilon$ & Grid G2 $k-\varepsilon$ & Grid2 RNG \\
\hline$c_{d}$ & 0.029035 & 0.019256 & 0.013741 \\
\hline Pressure $c_{d}$ & 0.019527 & 0.009465 & 0.004770 \\
\hline Friction $c_{d}$ & 0.009508 & 0.009791 & 0.008970 \\
\hline
\end{tabular}

Table 4.18: Components drag coefficients for NACA0015 section at $\alpha=6$ degrees, $\mathrm{Re}=1.2^{*} 10^{6}$, wetted flow

Form table 4.17 it is clear that the lift is mainly produced by the pressure 
force acting on the foil, while the friction force is negligible at a value of about $0.001 \%$ of the total lift force. Table 4.18 shows that pressure and friction drag components are both important contributors to the drag. While the friction drag is within the same range for all cases (around $0.0094+/-$ $5 \%$ ); the pressure drag varies with about $50 \%$ with grid (from G1 to G2 $k-\varepsilon$ turbulence model) and turbulence model (from standard $k-\varepsilon$ turbulence model to RNG $k-\varepsilon$ turbulence model). Further analyses of the pressure drag shows that the difference is mainly due to the pressure distribution at the leading edge suction side of the profile, thus related to the errors in $C_{p}$ predicted at the leading edge stagnation point.

In summary, the investigation of the 2D wetted flow over NACA0015, 6 degrees angle of attack and $R e=1.2^{*} 10^{6}$, a reveals relatively small influence of grid density and of the choice of turbulence model on pressure and lift coefficients. From the above investigation, the drag coefficient and stagnation pressure are the main parameters influenced by grid resolution and turbulence model. The closest to the estimate of the zero grid size solution is the solution obtained with the RNG $k-\varepsilon$ turbulence model. Thus, at this stage the results indicate that the RNG turbulence model is the most appropriate choice when considering steady flow performance for the current case. In the following the standard $k-\varepsilon$ and the RNG $k-\varepsilon$ turbulence models will be applied to a cavitating flow on the finest grid (G2).

\subsubsection{Results cavitating flow, $\sigma=1.0$}

The case of a cavitating flow at a cavitation number of $\sigma=1.0$ is characterized by a time-dependent shedding cavity (see [37]). A low cavitation number implies a large area of cavitating fluid and the appearance of the shedding of cavity clouds. Cavitation starts when the negative of the minimum pressure coefficient has a value equal to the cavitation number, i.e.: $\sigma=-C_{p m i n}$. From figure 4.17 we deduce that when the cavitation is activated the flow in the area where $\mathrm{Cp}$ is lower than 1 will cavitate. This criteria is met in a region of about $25 \%$ of the chord on the upper surface, assuming that the cavity ends where $-C_{p}<\sigma$, which in reality is not true.

Since numerical simulations for cavitating flows are time dependent simulations, the time step used for the numerical simulation is an important new parameter to be set. In the results that follow the time step used in the simulations is $t=10 \mu$ s and the turbulence inlet values are: turbulence intensity $I=1 \%$ and length $l=0.001 \mathrm{~m}$. Note that for the cavitating flow case a third turbulence model is used: the RNG $k-\varepsilon$ modified model as described in the Mathematical Modeling Chapter. 
In the following assessment, the effects of grid size, turbulence model and time step over the cavitating flow results are studied.

\section{Effect of grid density}

As for the case of wetted flow initially the standard $k-\varepsilon$ turbulence model is used over the two grids (G1 and G2) to assess the capability of this turbulence model to simulate unsteady cavitating flows. When analysing timedependent cavitating flows, a practical way of monitoring the convergence of the numerical simulations is by monitoring the cavity volume as a function of time. Please note that both presented simulations are converged within 10 inner iterations and have used a Courant number of about 0.3 and 0.15 , for grid G1 and grid G2, respectively where the time step $(\Delta t=1 E-05 \mathrm{~s})$ is similar. The Courant number $\left(C_{O}\right)$ is defined generally as:

$$
C_{O}=\frac{|\vec{v}| \Delta t}{L}
$$

where $|\vec{v}|$ and $L$ are a characteristic velocity and length, respectively and $\Delta t$ is the time step size, see [16].

In figure 4.19 (where $\mathrm{T}=0.0625 \mathrm{~s}$ ) the variation of the cavity volume in time is presented for the two investigated grids. In this case, the cavity is slowly increasing and decreasing periodically in time without shedding, with a cycle frequency of $4 \mathrm{~Hz}$. From this figure it is clear that the grid resolution, well within the recommended guidelines has almost no effect on the cavity cycle. When analysing the cavity obtained with the current settings, it is seen that a leading edge cavity starting at the leading edge is slowly increasing and decreasing, see 4.21, first column. This cavity pattern is not in agreement with the expected behaviour for this case, as follows from experimental observations and other simulations ([7] and [42]). Next, the influence of the turbulence model is investigated on the finest grid considered, i.e. G2 grid.

\section{Effect of turbulence modeling}

Already from the simulations for wetted (steady) flow the influence of the turbulence modeling has been observed. In the present subsection the influence of the standard $k-\varepsilon$, the RNG $k-\varepsilon$ and the RNG $k-\varepsilon$ modified turbulence model on the results of the numerical simulations for unsteady flow around NACA0015 is investigated. In figure $4.20(\mathrm{~T}=0.0625 \mathrm{~s})$ the cavity 


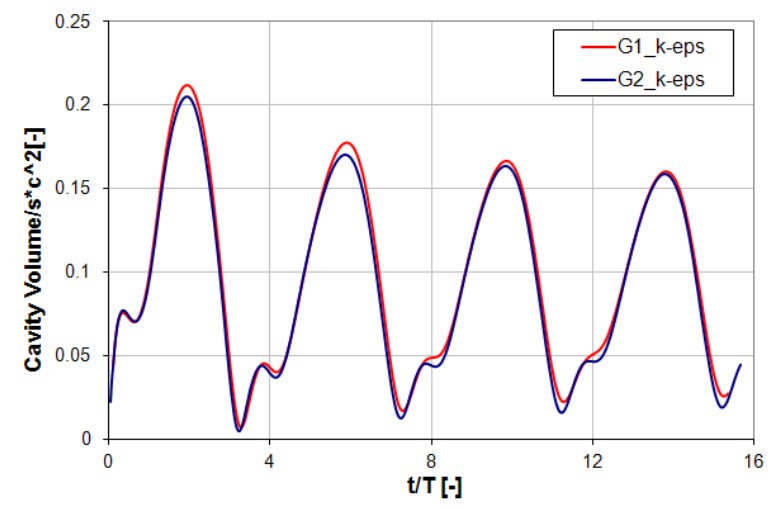

Figure 4.19: Cavity volume as function of time for flow condition with vapor cloud shedding, NACA0015, $\alpha=6$ deg, $\mathrm{Re}=1.2^{*} 10^{6}, \sigma=1.0, \mathrm{t}=1.0 \mathrm{E}-05 \mathrm{~s}$, standard $k-\varepsilon$ turbulence model, effect of the grid density

volume is presented as function of time for the different meshes and turbulence models. It can be observed that the standard $k-\varepsilon$ turbulence model gives the same cavity volume history on both meshes, while the RNG model predicts a larger cavity volume with higher frequency components in the time-dependent signal. Please note that all three presented results are converged solutions within 10 inner iterations and have a Courant number of about 0.1 , since the grid and time step $(\Delta t=1 E-05 \mathrm{~s})$ is similar.

When analysing the case of unsteady cavitating flow around the NACA0015 profile $(\sigma=1.0)$ the differences between the results obtained for different turbulence models used are clear. Contrary to the standard $k-\varepsilon$ simulations, for which the cavity is slowly increasing and decreasing periodically in time without shedding, for the RNG $k-\varepsilon$ and the modified RNG $k-\varepsilon$ turbulence models, the cavity becomes highly unsteady and the cavity starts to shed vapor clouds at a frequency of around $16 \mathrm{~Hz}$.

The FFT frequency analysis of the results of the time history of the cavity volume obtained from the numerical simulation with RNG and the RNG modified turbulence models reveals that the dominant frequencies, $15 \mathrm{~Hz}$ and $17 \mathrm{~Hz}$, respectively compare well. This frequency is well in agreement with experiments on NACA0015 performed by Arndt [7], in which a frequency of $16 \mathrm{~Hz}$ has been reported. In the literature shedding cycle frequencies from 12 to $24 \mathrm{~Hz}$ have been reported, both in measurements and predictions, see also Koop [42]. 


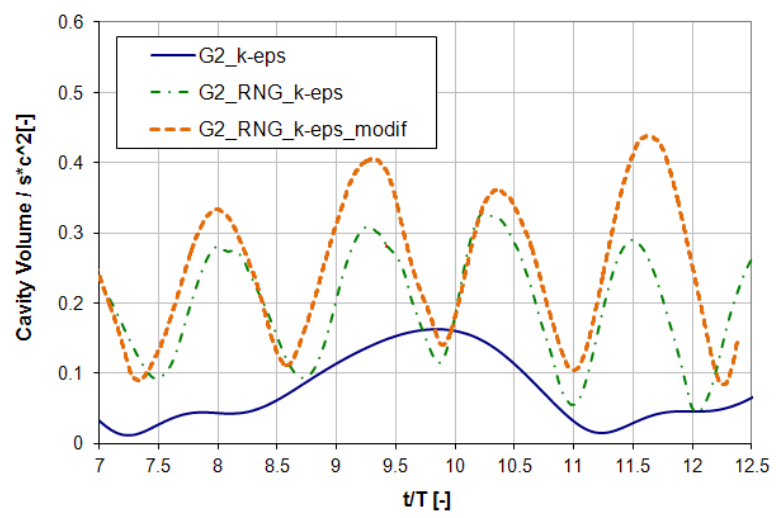

Figure 4.20: Cavity volume as function of time for flow condition with vapor cloud shedding, NACA0015, $\alpha=6 \mathrm{deg}$, Re $=1.2^{*} 10^{6}, \sigma=1.0$, grid G2, turbulence model dependency

Defining the Strouhal number based on the chord length as:

$$
S t_{c}=\frac{f c}{V}
$$

table 4.19 presents the results given in figure 4.20 and summarizes the first harmonic frequency expressed as the non-dimensional Strouhal number. In equation 4.27, $f$ is the frequency in $[\mathrm{Hz}], c$ is the chord length in $[\mathrm{m}]$ and $V$ is the free stream velocity in $[\mathrm{m} / \mathrm{s}]$.

\begin{tabular}{|c|c|c|c|}
\hline Mesh & Turbulence model & Frequency $[\mathrm{Hz}]$ & Strouhal number, $S t_{c}[-]$ \\
\hline G2 & $k-\varepsilon$ & 4 & 0.13 \\
\hline G2 & RNG $k-\varepsilon$ & 15 & 0.50 \\
\hline G2 & RNG $k-\varepsilon$ modif & 17 & 0.57 \\
\hline
\end{tabular}

Table 4.19: Frequency and Strouhal number for cavitating flow around NACA0015 section at $\alpha=6 \mathrm{deg}, R e=1.2 * 10^{6}, \sigma=1.0$

Figure 4.21 shows for one period of the shedding process the vapor volume fraction and velocity vectors for: standard $k-\varepsilon$ turbulence model, RNG turbulence model and modified RNG turbulence model.

Analysing the shedding behaviour in figure 4.21, the similarities are obvious. 


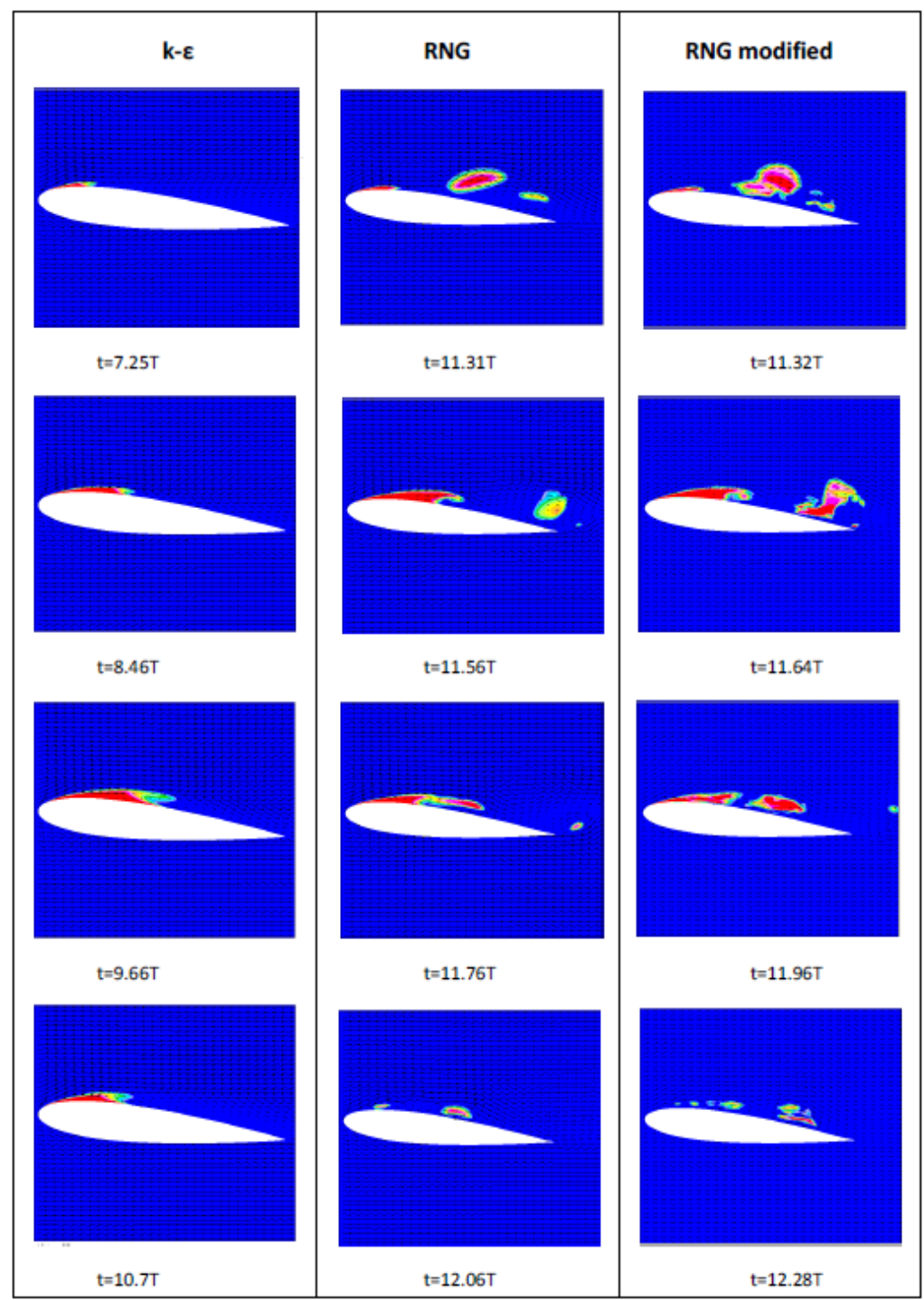

Figure 4.21: NACA0015 $\alpha=6 \mathrm{deg}, \operatorname{Re}=1197600, \sigma=1.0$, Contours of vapor volume fraction and velocity vectors during one shedding period. Effect of different turbulence models, grid G2 
In the macro structure of the cavities for the RNG turbulence models: the leading-edge cavity formation in the beginning of the cycle $(\mathrm{t} / \mathrm{T}=11.3)$, the re-entrant jet forming when the leading edge cavity reaches its maximum extent $(\mathrm{t} / \mathrm{T} \cong 11.6)$ and the detachment of vapor clouds due to the re-entrant jet $(\mathrm{t} / \mathrm{T} \cong 11.86)$. Nevertheless the differences between the results are also evident. When the turbulent viscosity correction is active within the mixture layer, the increase in richness in the structures of the cavitating flow is clear. The following presents a phenomenological description of the shedding process as follows from the results obtained with the modified RNG $k-\varepsilon$ turbulence model:

(i) a small leading edge cavity is observed at $\mathrm{t} / \mathrm{T}=11.3$ while at the same time shed vapor clouds from the previous period are present downstream of the leading edge

(ii) the leading edge cavity increases in size and the re-entrant jet starts to form at $\mathrm{t} / \mathrm{T}=\cong 11.6$; furthermore the vapor clouds from the previous cycle have travelled further downstream

(iii) at $\mathrm{t} / \mathrm{T} \cong 11.86$, the leading edge cavity starts to withdraw towards the leading edge, while the clouds are travelling and dissipate downstream

(iv) at $\mathrm{t} / \mathrm{T} \cong 12.18$, the leading edge cavity disappears while just a few vapor clouds are visible. In the next cycle, the leading edge cavity starts to grow again and the cycle is repeated from the beginning.

This cavity shedding pattern is confirmed in 3D experiments (developed to produce mainly $2 \mathrm{D}$ cavities). Furthermore, results of numerical simulations in [7] and [42] show the main phenomenological features as found in the present study.

In figures 4.22 and 4.23 , the velocity vectors and the corresponding contours of their magnitudes are plotted for the purpose of comparison for the RNG $k-\varepsilon$ and RNG $k-\varepsilon$ modified turbulence model. The figures show in more detail the differences between the results of the RNG models: the RNG modified turbulence model results in a higher velocity magnitude at the cavity interface and due to this more vortical structures are observed. This should be caused by the reduction in the turbulence viscosity due to the density modification from a "slow" linear decrease to a "steep" power decrease (see figure 2.1). Thus the dynamics of the flow are increased given: higher velocity magnitudes and more unsteadiness at the fluid-vapor interface, as observed in cavity contours. Still the differences are limited, the cavity and frequency 


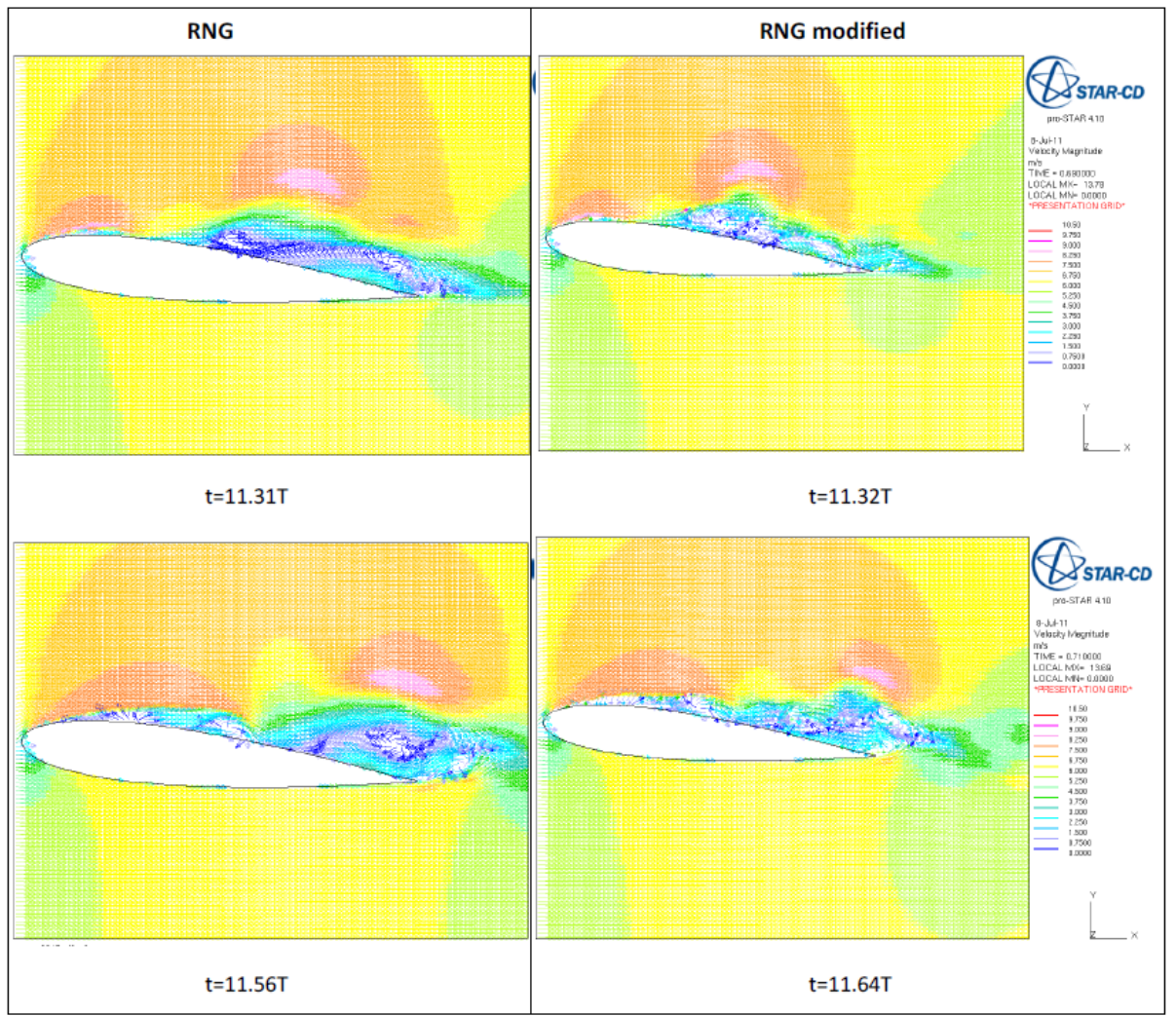

Figure 4.22: NACA0015 $\alpha=6 \mathrm{deg}$, Re $=1.2^{*} 10^{6}, \sigma=1.0$, Vectors coloured with velocity magnitude at $\mathrm{t} / \mathrm{T} \cong 11.3$ and $\mathrm{t} / \mathrm{T} \cong 11.6$. Effect of different turbulence models, RNG and RNG modified, grid G2

shedding are reasonably similar for both cases. Note that employing the SST $k-\omega$ turbulence model in Fluent, the cavity shedding was not observed (see [50]) without including the eddy viscosity correction.

Analysing the shedding period from the results for each turbulence model, the similarities and differences have been traced back to the development of the macro structure of the cavities: the formation of the leading edge cavity, the re-entrant jet and the detachment of the vapor clouds in the current section. 


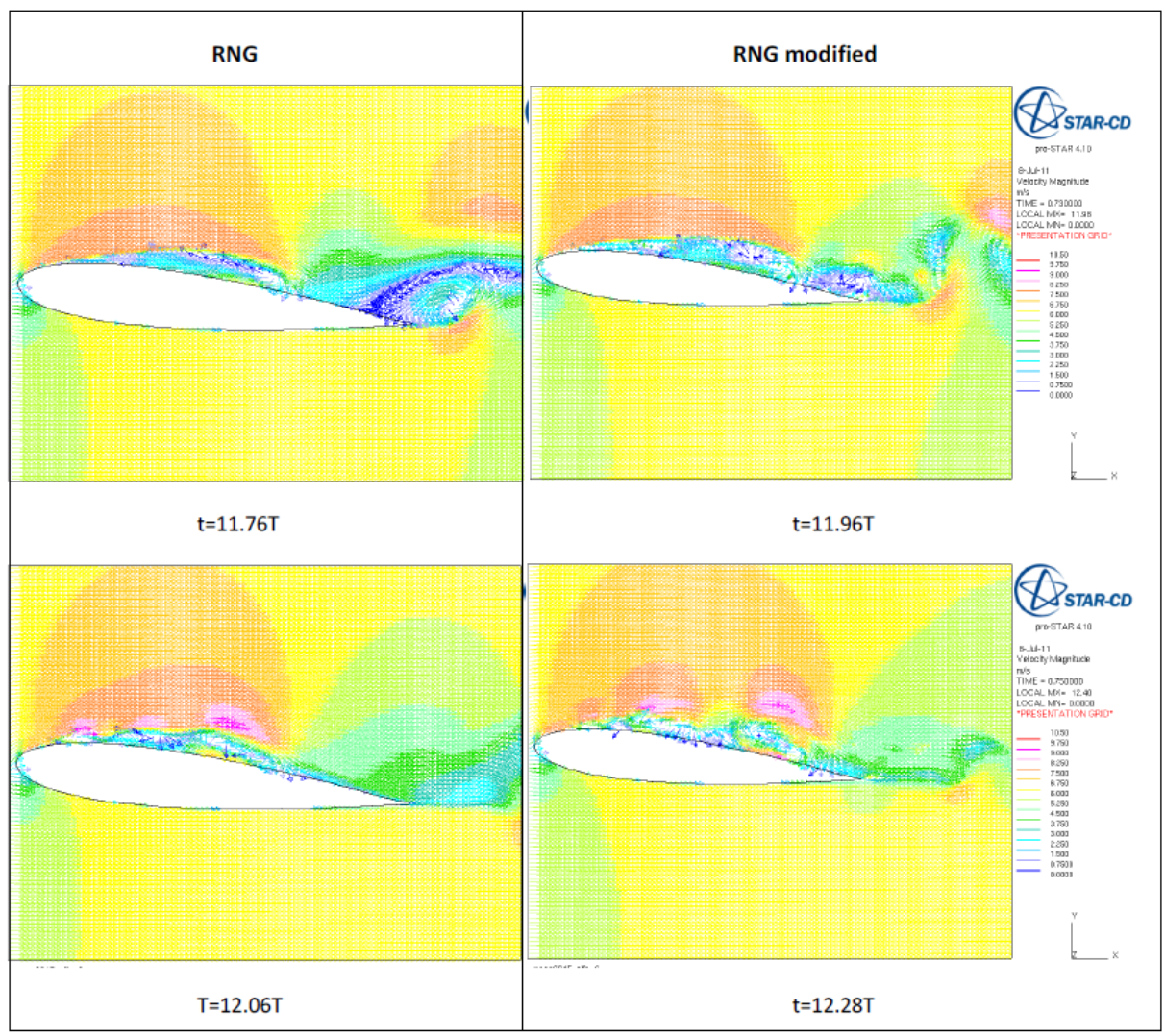

Figure 4.23: NACA0015 $\alpha=6$ deg, $\operatorname{Re}=1.2^{*} 10^{6}, \sigma=1.0$, Vectors coloured with velocity magnitude at $\mathrm{t} / \mathrm{T} \cong 11.8$ and $\mathrm{t} / \mathrm{T} \cong 12.1$. Effect of different turbulence models, RNG and RNG modified, grid G2

\section{Effect of time step}

The time integration method used is a first-order Euler implicit method as implemented in STAR-CD. In cavitating flow the physical time scale is so small that the numerical time step need to have a small values as well. Therefore it is assumed that a fast first-order method will give adequate temporal accuracy. 
The influence of the time step in the numerical simulation of unsteady flow over a NACA 0015 profile is investigated below. This is carried out by analysing the results for three time steps, specifically the cavity volume and the cavity shedding, for grid G2 and the RNG $k-\varepsilon$ turbulence model.

In the preceding studies on the unsteady flow over a NACA 0015, the time step used for all simulations equals $1 \mathrm{E}-05$ seconds. In the following, two extra time steps are investigated, one lower $(1.0 \mathrm{E}-04 \mathrm{~s})$ and one higher $(1.0 \mathrm{E}-06 \mathrm{~s})$ than the reference $(1.0 \mathrm{E}-05 \mathrm{~s})$. Note that the initial solution for these simulations is obtained from the solution for a time step of $1.0 \mathrm{E}-05 \mathrm{~s}$.

For the three time steps the variation of cavity volume in time is given in figure 4.24. The corresponding outer iterations per time step is given in figure 4.25. The corresponding Courant number as function of time based on the mean cell size for the three investigated time steps is presented in figure 4.26 .

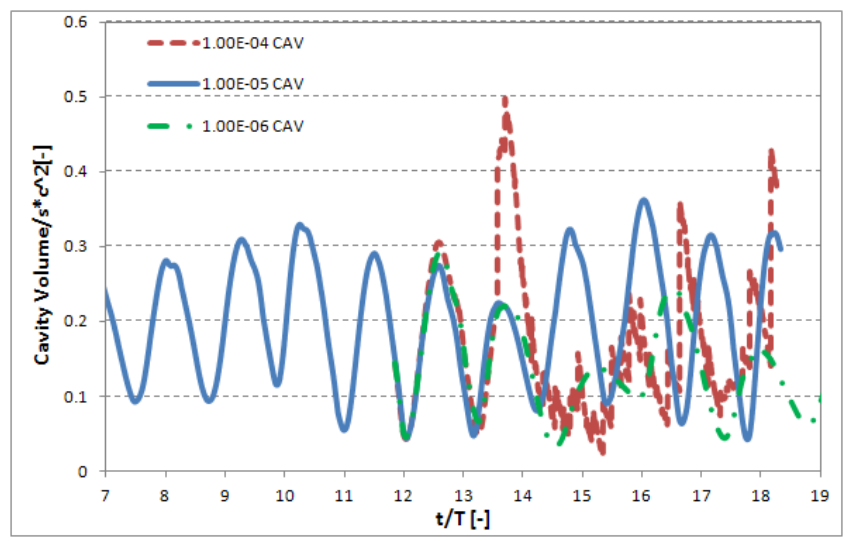

Figure 4.24: NACA0015 $\alpha=6 \mathrm{deg}, \operatorname{Re}=1.2^{*} 10^{6}, \sigma=1.0$, Non-dimensional cavity volume as function of time. Effect of time step, RNG turbulence model, grid G2

Analysis of the evolution of cavity volume for different time step reveals that:

(a) when $\Delta t=1 E-04 \mathrm{~s}$, the periodic variation in time of the cavity volume is lost and the overall cavity shedding phenomenon disappears. When analysing the number of outer iterations per time step as presented in figure 4.25 , it is obvious that for this case the simulations do not converge. The pressure equation requires more than 20 outer iterations, i.e. more than the recommended number of 10 to 20 as from 


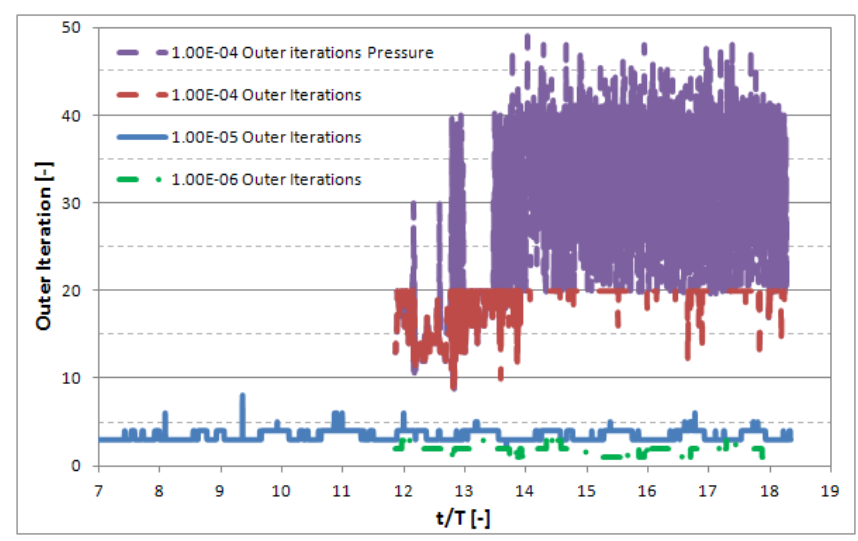

Figure 4.25: NACA0015 $\alpha=6$ deg, $\operatorname{Re}=1.2^{*} 10^{6}, \sigma=1.0$, number of outer iterations required for convergence. Effect of different time step, RNG turbulence model, grid G2

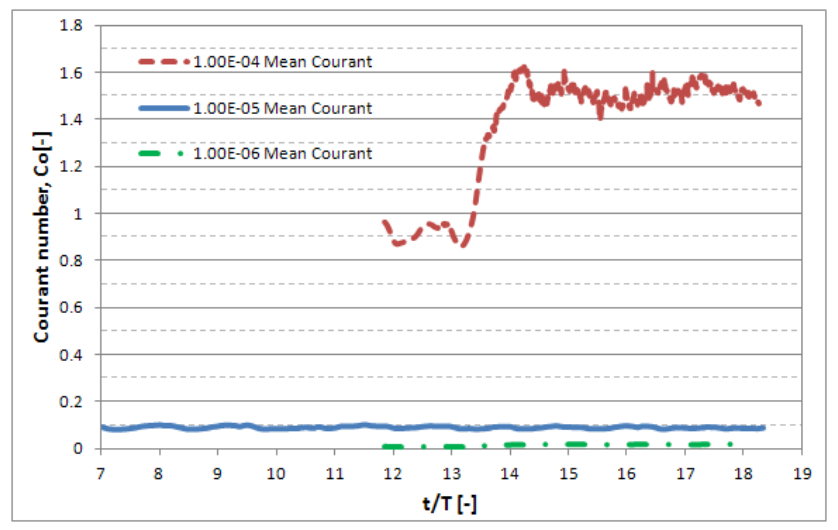

Figure 4.26: NACA0015 $\alpha=6 \mathrm{deg}, \mathrm{Re}=1.2^{*} 10^{6}, \sigma=1.0$, variation of Courant number with time. Effect of different time step, RNG turbulence model, grid G2

[16]. Also, the corresponding Courant number is much higher than the recommended value of around 0.3. This time step is not appropriate for this case of cavity shedding

(b) when $\Delta t=1 E-05 \mathrm{~s}$, the periodicity of the history of the cavity volume is captured at the expected shedding frequency and developing the 
expected cavity phenomenology described previously. Analysis of the number of outer iterations (4.25) and Courant number (4.26) do prove that the simulation convergence. The Courant number for this case is 0.1 , smaller than the recommended values of 0.3 (as from [16]), but closest to 0.3 , with respect to the Courant number of the other two investigated time steps (as from figure 4.26, 1.5 is the Courant number for $\Delta t=1 E-04 \mathrm{~s}$ time step and 0.01 is the Courant number for $\Delta t=1 E-06$ s time step)

(c) when $\Delta t=1 E-06 \mathrm{~s}$, the periodicity of the cavity volume and shedding is not lost and the cavity phenomenology still exists. The number of outer iterations (figure 4.25) shows that the numerical procedure for this case is convergent. The Courant number analysis (figure 4.26) shows a relatively low value which implies a very long simulation time. Compared to the previous time step $(\Delta t=1 E-05 \mathrm{~s})$, the frequency for this simulation is slightly reduced from 14 to $15 \mathrm{~Hz}$ to around 12 $\mathrm{Hz}$, but still in-line with the shedding frequency found in literature, namely between 12 and $24 \mathrm{~Hz}$, see [42]. Also, in this case the amplitude is oscillates more then compared to the previous case; this time step may need longer simulations time

Based on this study, it is clear that the time step has to be selected in such way that the number of outer iterations does not exceed 10 to 20 (as from [16]) and with a reasonable Courant number (around 0.3 in the present case). If the number of outer iterations required is higher than the recommended value, it is recommended that the time step should decrease and the number of outer iteration should not be increased, see [16]. Also, from [17]: "for optimal results with respect to interface sharpness (resolution within one to two cells), the Courant number should be around 0.3".

For the investigated grid and the recommended Courant number, the time steps of $1 \mathrm{E}-05 \mathrm{~s}$ and $1 \mathrm{E}-06 \mathrm{~s}$ are suitable to capture the shedding phenomenon at the expected frequency.

But from the definition of the Courant number in equation 4.26 it follows that two unwanted situations arise:

(1) If the time step $(\Delta t)$ is larger and the grid $(L)$ is small, the resulting Courant number is large, as in the results for the time step 1E-04s. In this case the numerical simulations are not able to capture the cavity shedding precess

(2) If time step $(\Delta t)$ is small and the grid $(L)$ is large, the Courant number is small, as in the case corresponding to the time step of $1 \mathrm{E}-06 \mathrm{~s}$. In 
this case the simulations are still able to predict the shedding cavity but within very large time periods, up to 10 to 20 time higher than the case of $\Delta t=1 \mathrm{E}-05 \mathrm{~s}$.

The ideal case for the proper grid (in the current case grid G2) and the proper time step $(\Delta t=1 E-05 \mathrm{~s})$ gives the recommended Courant number (around $0.3)$ for the case of cavitating flow around NACA 0015 profile.

\subsubsection{Unsteady Flow Conclusions: Turbulence and Time step Guidelines}

The simulations for steady wetted flow over the NACA0015 profile, show that different meshes and turbulence models lead to comparable results (within $5 \%$ ) in terms of surface pressure distribution and lift coefficient. The results produced employing the RNG turbulence model are slightly closer to the correct value of the stagnation pressure, but also in terms of the drag coefficient. The simulations for unsteady cavitating flow show through the assessment made for the effect of variation of grid, turbulence model and time step, that the time step is of utmost importance when grid convergence is to be reached. It should be emphasized that mesh resolution is also very important but when grid convergence is reached (as both meshes comply with the grid guidelines resulting from the study of the Elliptic 11 rake hydrofoil) and the results are still not satisfactory that then the turbulence model turns out to be very important when dealing with cavitating flows. The latter situation is clearly shown in the present study.

Modeling errors, due to e.g. turbulence and cavitation model, are becoming important when dealing with cavitating flows.

Thus, once grid convergence has been reached and an adequate turbulence model has been selected, the time step has a strong effect on the cavity shedding phenomenon of the NACA 0015 profile. If a too large time step is used the flow becomes steady and the simulations do not converge within 10 to 20 outer iterations. Lowering the time step, the cavity shedding period, expected from the test case is found for $\Delta t=1 E-05 \mathrm{~s}$. Please note that a smaller time step still influences the shedding frequency slightly, without affecting the character of the overall shedding process. The main drawback of using a very small time step is that the simulations time increases to 10 to 20 times the simulation time for a larger time step for which the cavity shedding and frequency is still reasonably predicted. Note also that once grid convergence has been reached, this will not further influence the cavity shedding process. 
The cavity shedding patterns found for the 2D NACA0015 section for $\sigma=1$ are largely confirmed by experiments in 2000 (see [7]) and numerical simulations for 2D flow in 2008 (see [42]). Using the RNG $k-\epsilon$ turbulence model (with or without turbulent viscosity modification), a grid converged solution for a acceptable time step (for a suitable Courant number) is observed for the cavitating flow around the NACA 0015 section.

\subsection{Conclusions}

The grid requirements for steady flow simulations have been assessed through analysing the flow over the Elliptic 11 rake hydrofoil. The minimum average value required for the surface mesh assures numerical results within $5 \%$ from the estimated grid-converged values. These grid-sizes will be used as requirement for the grids constructed for the test cases combined with an adequate $y^{+}$value. In case special features are encountered a successive grid refinement will be applied.

These grid guidelines for the surface mesh will be verified for each test case considered.

The investigation of unsteady flow performed for the NACA0015 section has revealed the importance of the mesh refinement (beyond the mesh guidelines), and in addition the choice of the turbulence model for steady flow and the time step in unsteady flow.

The standard $k-\varepsilon$ turbulence model, works adequately for wetted flow, however, for the prediction of drag and the distribution of the pressure coefficient the RNG $k-\varepsilon$ turbulence model is recommended.

For unsteady flows, the standard $k-\varepsilon$ turbulence model is not adequate for the case of cavitation, characterised by the occurrence of strong vortical flow. A more advanced model like the RNG $k-\varepsilon$ turbulence model has proven to be more suitable for predicting the shedding frequency.

Note that all simulations have been performed under the assumption of incompressible flow. However, the vapor present in cavitating flows implies that compressibility will affect the flow. By using a relatively simple density modification within the relation for the turbulent viscosity (as described in Mathematical Modeling Chapter) this effect is accounted for in the RNG modified turbulence model, as recommended in [24] and [86]. When applying the modified RNG turbulence model, the cavitating flow becomes more unsteady with a higher velocity gradient at the liquid-vapor interface. Associated with this are much higher values for the vorticity (due to the compressibility of the mixture). Still for the cavitating flow over the NACA0015 
section the RNG turbulence model, with and without density modification, produces shedding cavities that have been observed in 3D experiments. This is also the case for results of grid-converged numerical simulations employing a proper time step (that complies with the recommendations for the Courant number).

The numerical simulations reported on in this thesis will comply with the grid and time step requirements and both RNG and RNG modified turbulence model will be used. 


\section{Chapter 5}

\section{Underlying Flow Characteristics}

\subsection{Introduction}

The objective of this chapter is to study the performance of the method for cavitating flows and its capability to predict the mechanisms of developed cavitation, like sheet cavitation and leading edge-tip vortex cavitation. The purpose is to use the RANS approach to predict cavitating flows as occurs in typical 3D test cases.

Two benchmark test cases are analysed in the present chapter:

1. The case of the flow over a 3D twisted hydrofoil geometry featuring 3D sheet cavitation. This case, representative for a complex leading-edge shedding cavity, will indicate the capability of the method to predict: the complex shedding cavitation phenomenon, the shedding frequency and possibly the location of cavitation erosion.

2. A 3D elliptic wing, representative for a complex interaction of the sheet cavity and tip vortex cavitation, frequently present on marine propellers. This will indicate the capability of the method to predict: the developed leading edge-tip vortex cavitation interaction, the cavitating tip vortex core and the far field wake development of the tip vortex cavitation.

From the chapter on the Uncertainty Study guidelines have been found for the grid (from the Elliptic 11 rake hydrofoil wetted flow) and for the time step (from the unsteady flow around the NACA0015 profile). Only the choice of an appropriate turbulence model is still an open issue (in the cavitating flow case) since both the RNG $k-\varepsilon$ and the RNG $k-\varepsilon$ modified turbulence 
model appear to perform as expected.

The numerical simulations reported on in the current chapter are performed for wetted and for cavitating flow conditions. The influence of the local grid refinement (like tip vortex resolution in the Elliptic 11 rake hydrofoil) and turbulence modelling for cavitating flow conditions are assessed in the following. At the end of the chapter, guidelines are given for the appropriate turbulence modeling. Furthermore, requirements for the grid resolution are provided when are necessary for the tip vortex and to predict different types of cavitation and their associated features.

Our guiding principle is that a complex flow can be predicted sufficiently reliably when the underlying flow mechanisms are predicted correctly. To achieve this, the quality of the prediction of the basic flow mechanism will be studied through two benchmark cases:

1. Delft Twist 11 hydrofoil: to study the dynamic break up of the sheet cavitation.

2. Elliptic 11 rake hydrofoil: to study the extent of the sheet and tip vortex and their dynamics.

It is hypothetical that only when these proposed benchmark test cases (the Twist 11 hydrofoil and the Elliptic 11 rake foil) designed to generate the most basic cavitation features are completed successfully, that only then method developed for numerically simulating cavitating flows can be used for prediction of propeller tip vortex cavitation: the goal of the thesis. Thus, each of the following test cases is crucial since the resulting knowledge is essential for the next step of the thesis.

\subsection{Delft Twist-11 hydrofoil}

\subsubsection{Geometry}

The geometry for this test case has been designed to produce a mid-span cavity which forms and detaches periodically, see [29]. The hydrofoil spans the water channel and has a NACA0009 section with a span-wise varying geometric angle of attack such that the wall effects on the sheet cavity dynamics are minimized, i.e. a low local angle of attack at the channel walls such that cavity does not occur near the channel walls. To obtain a low pressure zone around the mid-span region of the 3D hydrofoil the angle of attack distribution along the span is given by: 


$$
\alpha(\bar{y})=\alpha_{\max }\left(2|\bar{y}|^{3}-3 \bar{y}^{2}+1\right)+\alpha_{\text {wall }}
$$

In equation (5.1) $\alpha_{\max }=11$ degrees and $\alpha_{\text {wall }}=-2$ degrees, $\bar{y}=y /(b / 2)$ and $b$ is the full span of the left-right symmetric hydrofoil. The dimensionless span-wise coordinate is $\bar{y}=[-1,1]$ with $\bar{y}=0$ at mid-span and -1 and 1 at the left and right tunnel wall, respectively.

Figure 5.1 shows the detailed span-wise distribution of the geometric an-

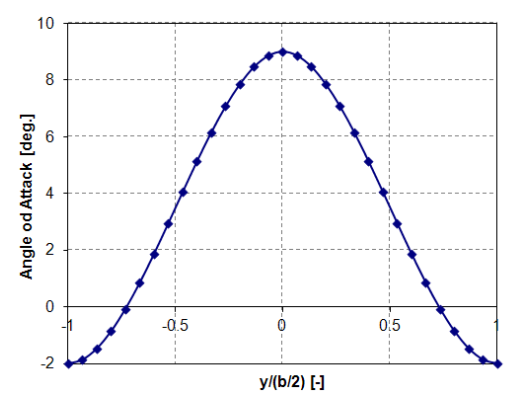

Figure 5.1: Span-wise distribution of geometric angle of attack of Twist 11 hydrofoil. $\alpha_{\max }=11$ degrees and $\alpha_{\text {wall }}=-2$ degrees

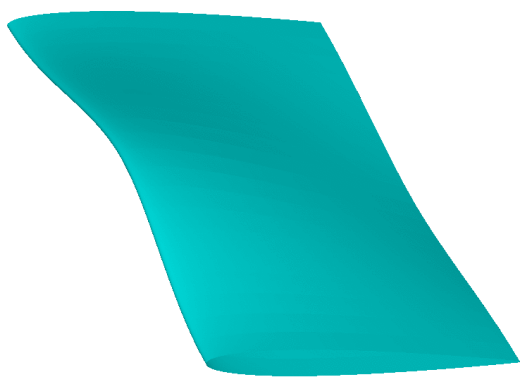

Figure 5.2: Surface geometry of Twist 11 hydrofoil. $\alpha_{\max }=11$ degrees and $\alpha_{\text {wall }}=-2$ degrees

gle of attack. The chord length of the hydrofoil is $0.15 \mathrm{~m}$ and the span is $\mathrm{b}=0.3 \mathrm{~m}$. Note that the hydrofoil section rotates around $\bar{x}=x / c=0.75$. The described hydrofoil is referred to as the Twist 11 hydrofoil and for more in-sights see [42]. 
The surface geometry of the 3D Twist 11 hydrofoil is shown in figure 5.2.

\subsubsection{Computational domain and mesh}

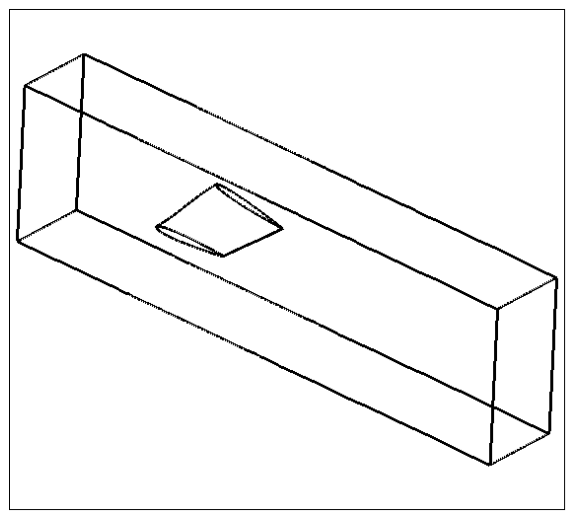

Figure 5.3: Computational domain for Twist-11 hydrofoil (half of the geometry)

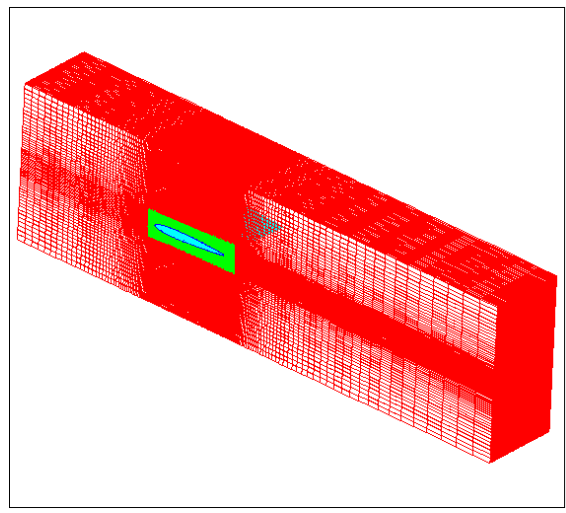

Figure 5.4: Mesh for Twist-11 hydrofoil

The experiments were performed in 2008 in the water tunnel at Delft University of Technology, see [29] for details. The dimensions of the test section 


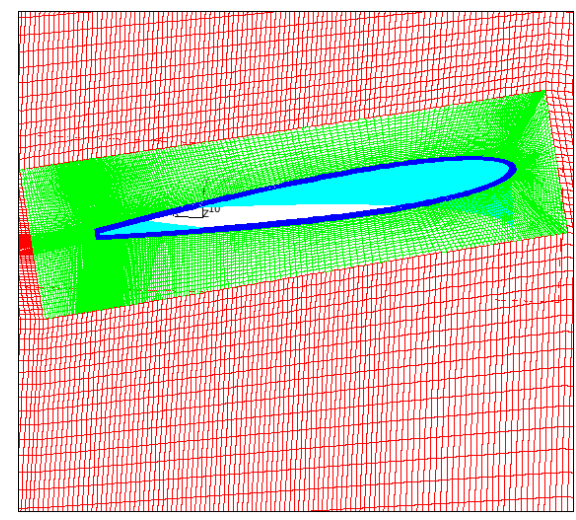

Figure 5.5: Mesh for Twist-11 hydrofoil, zoom O-grid

are $0.3 \mathrm{~m}$ by $0.3 \mathrm{~m}$. The length of the test section is $0.6 \mathrm{~m}$. For the computational domain as recommended in the EU Virtue project [37], the domain is extended to two chords ahead of the leading edge of the hydrofoil and 4 chords behind its trailing edge, see figure 5.3. The mesh for the 3D Twist-11 is similar to the one used for the NACA0015 configuration: a multi-block structured grid with an O-grid type around the chord-wise section, extruded in the span-wise direction with geometric angle of attack as shown in figure 5.1. The final mesh consists of 3 millions cells with about 2 millions cells for the O-grid type around the foil, see figure 5.5. Derived in the Uncertainty Study Chapter, the recommended non-dimensional chord-wise and span-wise averaged surface mesh size is about $0.007 \mathrm{c}$ and $0.017 \mathrm{~s}$, respectively. The cell length and width are normalised with the foil chord c and span s, respectively. For the mesh used in the present study the average chord-wise mesh is $0.005 \mathrm{c}$ and span-wise $0.007 \mathrm{~s}$, respectively; thus well in line with the recommendations (the fine mesh in span-wise direction is desired for this test case because of the substantial span-wise variation of the flow). The fine mesh around the hydrofoil should capture small cavity clouds expected for this case as observed in the experiments by Foeth [29].

\subsubsection{Numerical parameters}

Given the computational domain and its mesh, the boundary conditions are imposed at the sides of the domain as in table 5.3.

It has been assumed that the flow around the geometrically left-right sym- 
metric hydrofoil is also symmetric. This implies that only half of the hydrofoil needs to be considered, which reduces the number of cells to stay within the limits of the hardware. The hydrofoil surface is treated as wall and the standard wall function approach is used.

As for the NACA0015 section, the RANS equations are solved using the implicit SIMPLE algorithm. The spatial discretization is second-order accurate using the MARS scheme.

\begin{tabular}{|c|c|}
\hline & Boundary type \\
\hline Inlet & Inlet velocity \\
\hline Outlet & Pressure \\
\hline Tunnel walls & Slip \\
\hline Mid plane $(y=0)$ & Symmetry \\
\hline
\end{tabular}

Table 5.1: Boundary conditions computational domain Twist 11 hydrofoil

\subsubsection{Results for wetted flow}

The flow conditions for the wetted flow over the Twist 11 foil are identical to those in the experiments:

\begin{tabular}{|c|c|}
\hline Boundary Type & Value \\
\hline Inlet Velocity & $6.75 \mathrm{~m} / \mathrm{s}$ \\
\hline Outlet Pressure & $97.0 \mathrm{kPa}$ \\
\hline
\end{tabular}

Table 5.2: Boundary values for the Twist 11 hydrofoil configuration $\left(\alpha_{\max }=\right.$ $11 \mathrm{deg}$ and $\left.\alpha_{\text {wall }}=-2 \mathrm{deg}\right), \mathrm{Re}=1.01 \mathrm{E}+06$

This corresponds to a Reynolds number based on the chord length of $\mathrm{Re}=1.01 \mathrm{E}+06$. In the experiments, the Twist 11 hydrofoil was equipped with 12 pressure taps. Except for one sensor which is located on the pressure side, all sensors are located on the suction side. In figures 5.6, 5.7 and 5.8, the computational results (lines) are compared with the measured chord-wise pressure distribution (dots) at three span-wise locations. The measured data indicate also the $5 \%$ error bars. The figures show a good agreement between the experimental data and the results of the numerical simulations for wetted flow.

The hydrofoil was also mounted in a two-component load cell at both tunnel walls so that the force could be measured. For different velocities, the lift 
component was measured and fitted with the following quadratic relation for lift: $L=10.052 V^{2}$ with a corresponding $r^{2}$ value of 0.9455 , so that lift coefficient is $C_{L} \equiv L / 0.5 \rho V^{2} S$ with $S=0.3 \times 0.3 \mathrm{~m}^{2}$. Measured and predicted lift coefficients are shown in table 5.3. The difference between the curve fit and measured values is about $\pm 5.5 \%$ over the entire velocity range.

It can be concluded that for this configuration the method can predict $3 \mathrm{D}$ wetted flow with reasonable accuracy, within the $5 \%$ experimental uncertainty.

\begin{tabular}{|c|c|c|}
\hline Name & Measurement & CFD Simulation \\
\hline$C_{L}$ & $0.43875 \pm 5.5 \%$ & 0.41635 \\
\hline
\end{tabular}

Table 5.3: Lift Coefficient for Twist 11 hydrofoil configuration $\left(\alpha_{\max }=11\right.$ deg and $\left.\alpha_{\text {wall }}=-2 \mathrm{deg}\right), \mathrm{Re}=1.01 \mathrm{E}+06$, measured and predicted values

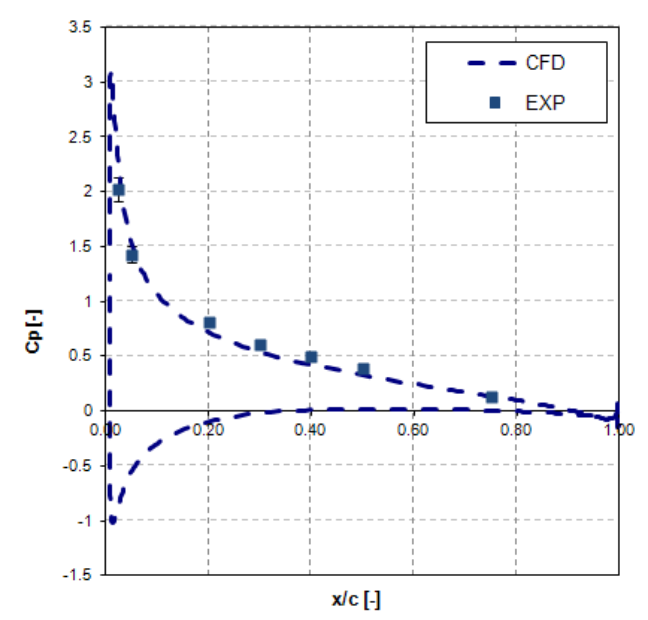

Figure 5.6: Predicted and measured Cp-distribution at $y=0$, Twist 11 hydrofoil configuration $\left(\alpha_{\max }=11 \mathrm{deg}\right.$ and $\left.\alpha_{\text {wall }}=-2 \mathrm{deg}\right), \operatorname{Re}=1.01 \mathrm{E}+06$, wetted flow 


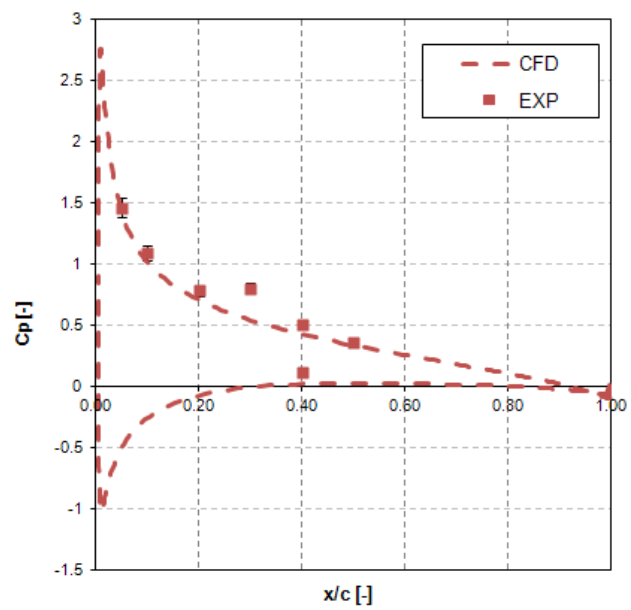

Figure 5.7: Predicted and measured Cp-distribution at $y /(b / 2)=0.2$, Twist 11 hydrofoil configuration $\left(\alpha_{\max }=11 \mathrm{deg}\right.$ and $\left.\alpha_{\text {wall }}=-2 \mathrm{deg}\right)$, $\mathrm{Re}=1.01 \mathrm{E}+06$, wetted flow

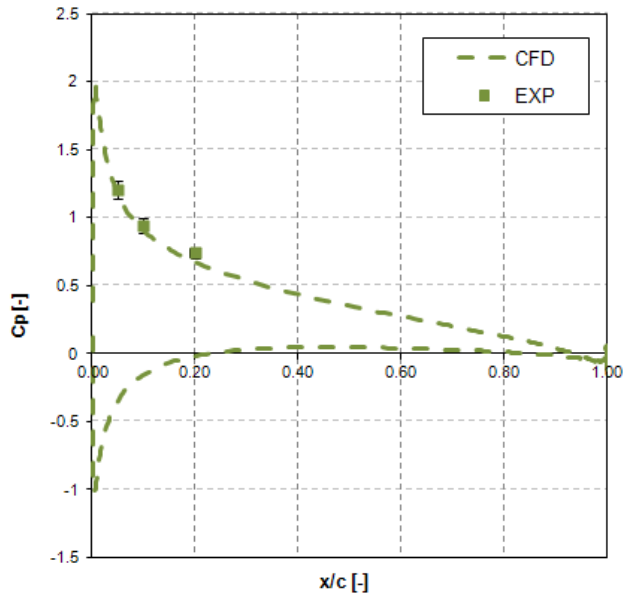

Figure 5.8: Predicted and measured Cp-distribution at $y /(b / 2)=0.4$, Twist 11 hydrofoil configuration $\left(\alpha_{\max }=11 \mathrm{deg}\right.$ and $\left.\alpha_{\text {wall }}=-2 \mathrm{deg}\right)$, $\mathrm{Re}=1.01 \mathrm{E}+06$, wetted flow 


\subsubsection{Results for cavitating flow, $\sigma=1.0$}

The case of the cavitating flow, typically occurring when the minimum surface pressure drops below the vapour pressure, is analysed in the present section. This test case is performed for conditions similar to the ones in the experiment, as listed in table 5.4.

\begin{tabular}{|c|c|}
\hline & Values \\
\hline Inlet Velocity & $6.97 \mathrm{~m} / \mathrm{s}$ \\
\hline Outlet Pressure & $29 \mathrm{kPa}$ \\
\hline Saturation Pressure & $2970 \mathrm{~Pa}$ \\
\hline Temperature & $297 \mathrm{~K}$ \\
\hline Vapor Density $\rho_{v}$ & $0.023 \mathrm{~kg} / \mathrm{m}^{3}$ \\
\hline Water Density & $998 \mathrm{~kg} / \mathrm{m}^{3}$ \\
\hline
\end{tabular}

Table 5.4: Flow conditions, Twist 11 hydrofoil configuration $\left(\alpha_{\max }=11 \mathrm{deg}\right.$ and $\left.\alpha_{\text {wall }}=-2 \mathrm{deg}\right), \operatorname{Re}=1.01 \mathrm{E}+06$, cavitating flow $\sigma=1.0$

The time step for the cavitating flow simulations is $\Delta t=10 \mu \mathrm{s}$, conform to the guideline from the analysis of the 2D NACA0015 profile case uncertainty study, presented in Chapter 4.

In the case of $2 \mathrm{D}$ cavitating flow the choice of the turbulence model played an important role: only the RNG turbulence model could predict shedding cavities. In the following the standard $k-\varepsilon$, the RNG $k-\varepsilon$ and RNG $k-\varepsilon$ modified turbulence models are employed and their effect on the $3 \mathrm{D}$ cavitating flow is analysed. The results for the cavitating flow for the Twist 11 hydrofoil are obtained for a single mesh that satisfies the grid guidelines from the Uncertainty Study Chapter.

After the initial phase, the convergence behaviour is analysed by monitoring the cavity volume as function of time. Figure 5.9 shows the history of the cavity volume as obtained for the $k-\varepsilon$, RNG $k-\varepsilon$ and RNG $k-\varepsilon$ modified turbulence models. For the 2D case both RNG $k-\varepsilon$ turbulence models predicted similar cavity volumes, with a slightly higher volume obtained for the RNG $k-\varepsilon$ modified turbulence model. For the 3D case the difference in results of the RNG $k-\varepsilon$ and RNG $k-\varepsilon$ modified turbulence models is much more distinct. The cavity contours and iso-contours obtained from the results for the standard $k-\varepsilon$ and RNG $k-\varepsilon$ turbulence models predict a mid-chord cavity slightly oscillating in time, similar to the results for the $k-\varepsilon$ turbulence model in the 2D case. However, the RNG $k-\varepsilon$ modified turbulence model produces a complex mid-span cavity that forms and detaches periodically in time. 
It follows from figure 5.9 that the cavity shedding period, using the RNG modified turbulence model, equals 0.032 seconds, which gives a frequency of $31 \mathrm{~Hz}$. This frequency for the shedding matches well with the experimentally determined shedding frequency of $33 \mathrm{~Hz}$, see [29]. Figures 5.10 and 5.11 present a comparison of the numerical results with experimental results. This shows a good match between the experimental observations and numerical simulations at four analysed time steps.

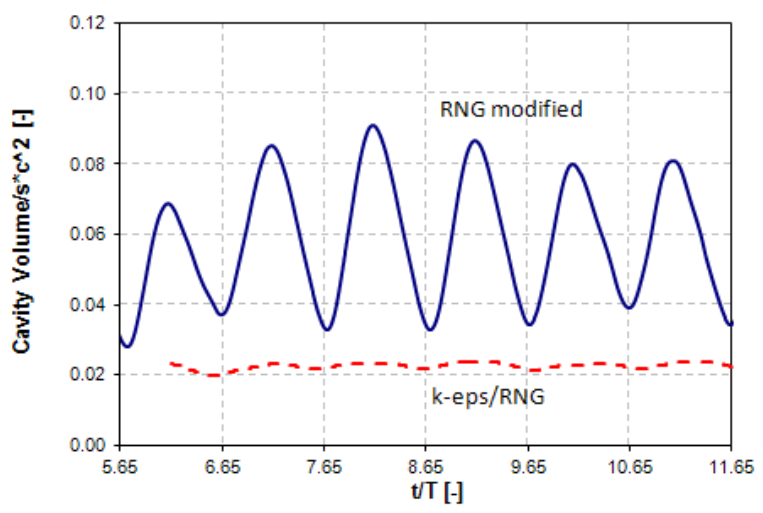

Figure 5.9: History of the cavity volume, Twist 11 hydrofoil configuration $\left(\alpha_{\text {max }}=11 \mathrm{deg}\right.$ and $\left.\left.\alpha_{\text {wall }}=-2 \mathrm{deg}\right), \operatorname{Re}=1.01^{*} 10^{6}\right)$, cavitating flow $\sigma=1.0$

From the numerical results iso-surface plots of a vapor concentration of 0.01 (1\% vapor content) have been generated. Results for the following times are presented and analysed in the following for the 7 th cycle: $\mathrm{t} / \mathrm{T}=6.65, \mathrm{t} / \mathrm{T}=6.9$, $\mathrm{t} / \mathrm{T}=7.28$ and $\mathrm{t} / \mathrm{T}=7.56$. At the first time step $(\mathrm{t} / \mathrm{T}=6.65)$, a large leading edge cavity is shown, which agrees with the photo of the experiments. The second picture $(\mathrm{t} / \mathrm{T}=6.9)$ shows the start of the break-up of the cavity at mid-span, a large cavity cloud starts to detach from the main leading edge cavity and the side entrant jets are formed. In the third picture at $\mathrm{t} / \mathrm{T}=7.28$ the detached cloud has travelled downstream and has a shape of a so called 'horse shoe' cavity, see also [74] and [83]. Also the main leading edge cavity starts to recover to its maximum length in preparation for the next cycle of cloud detachment. In the fourth picture at $\mathrm{t} / \mathrm{T}=7.56$, the remaining vapor clouds are travelling downstream, while the leading edge cavity continues to grow and the cycle is repeated from the top. 

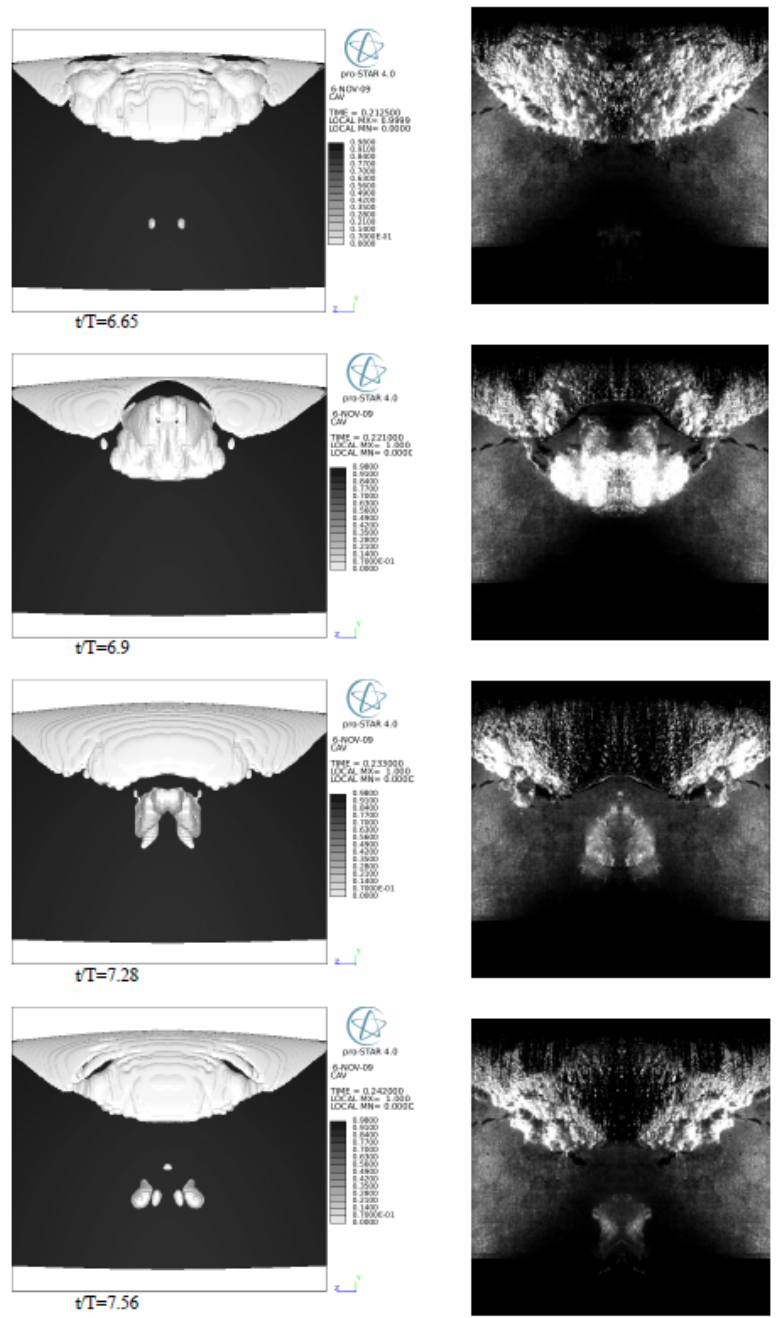

Figure 5.10: Twist 11 hydrofoil configuration $\left(\alpha_{\max }=11 \mathrm{deg}\right.$ and $\alpha_{\text {wall }}=-2$ $\mathrm{deg}, \operatorname{Re}=1.01^{*} 10^{6}$, cavitating flow $\left.\sigma=1.0\right)$. Iso-surface of vapor fraction $=0.01$ during one period. Comparison of predictions (left) and experiment of Foeth [29] (right), top view 

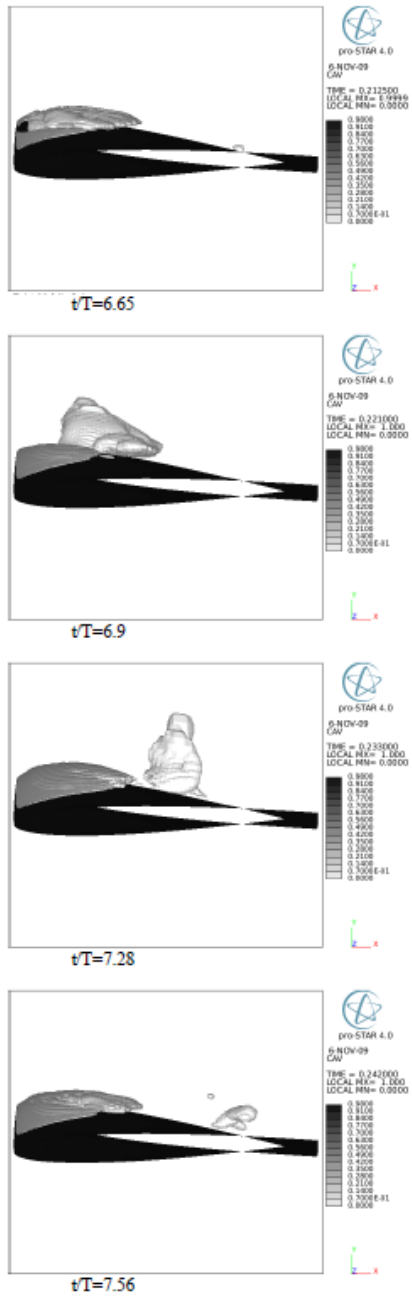
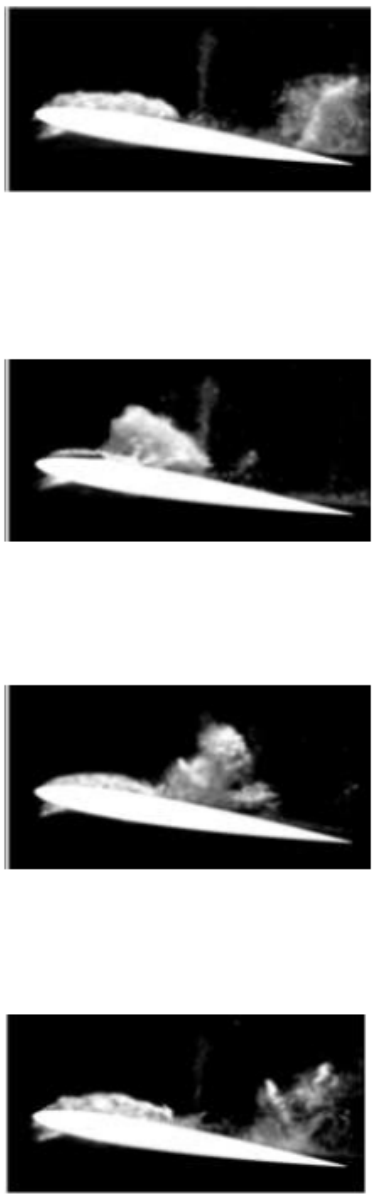

Figure 5.11: Twist 11 hydrofoil configuration $\left(\alpha_{\max }=11 \mathrm{deg}\right.$ and $\alpha_{\text {wall }}=-2$ $\mathrm{deg}, \mathrm{Re}=1.01^{*} 10^{6}$, cavitating flow $\left.\sigma=1.0\right)$. Iso-surface variation of the vapor fraction $=0.01$ during one period. Comparison of predictions (left) and experiment of Foeth [29] (right), side view 
The agreement between the results of the numerical simulations and the photos from the experiments is quite satisfactory for all four time steps in terms of the cavity macro structure (cavity extent) and dynamics. What is missing in the results of the simulations are the small-scale cavity structures which appear abundant in the experiments. Still, based on the present results it is concluded that the RNG $k-\varepsilon$ modified turbulence model is able to simulate large scale phenomena including the complex 3D sheet cavity shape and its cyclic shedding behaviour. Also the prediction of the lowest natural frequency of this phenomenon is in good agreement with the corresponding experimental value.

A more detailed validation of the cavitating flow mechanism can be made by considering, at the mid-foil section, the vapor fraction contours and velocity vectors at four representative time steps in a cycle. In left-hand side of the figure 5.12 the vapor fraction and the velocity vectors are shown for time step $\mathrm{t} / \mathrm{T}=6.65$, when the leading edge cavity has the largest extent. At this point in time the re-entrant jet is very strong reaching almost the leading edge of the hydrofoil and a cloud is already starting to detach at the aft part of the cavity. In the right-hand side of figure 5.12, the cloud detached from the leading edge, is travelling downstream while a new leading edge cavity starts to form. Note that associated with the detachment the flow starts to rotate, i.e. a vortical structure is formed, partially containing vapor. In the third step at $\mathrm{t} / \mathrm{T}=7.28$, the left-hand side of figure 5.13 , the leading edge cavity has grown while the cloud is shrinking while travelling further downstream in to the region with higher pressure. Finally, in the right-hand side of the figure 5.13, the re-entrant jet has formed again and starts to travel towards the leading edge to detach the next cloud. This mechanism was also observed from the top view of the cavity iso-surface contour.

The nuisance due to cavitation is mainly the possible erosion of the surface material, the radiated noise and the pressure fluctuations. These unwanted effects are produced due to the cavitation induced pressure waves / fluctuating pressures generated by the collapse of cavitation clouds and / or micro jets generated upon bubble collapse close to the solid surface, see [29] and [11]. However, the micro-jets were argued not to be the main cause for cavitation erosion (Van Terwisga et al. 2009, see [83]). The variasion of the hydrofoil lift during one period of cavity shedding is shown in figure 5.14. Figure 5.14 displays two regions with a reduction in lift, observed at $\mathrm{t} / \mathrm{T}=6.9$ and at $\mathrm{t} / \mathrm{T}=7.56$. At $\mathrm{t} / \mathrm{T}=6.9$, the surface pressure distribution is shown in one representative normal plane in the left-hand side of the figure 5.15. For $\mathrm{t} / \mathrm{T}=7.56$, the right-hand side of the figure 5.15 shows the corresponding pressure distribution. 

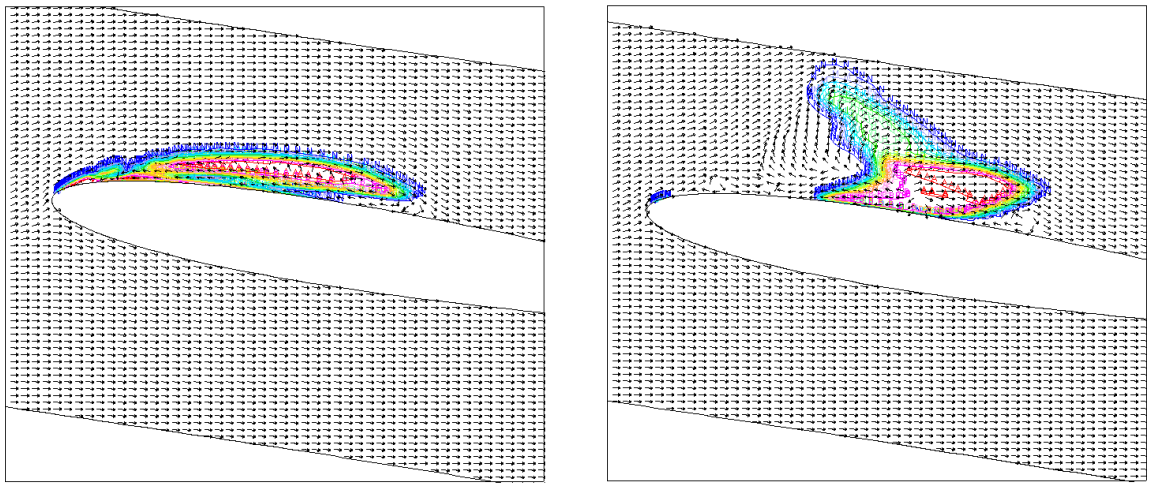

Figure 5.12: Twist 11 hydrofoil configuration $\left(\alpha_{\max }=11 \mathrm{deg}\right.$ and $\alpha_{\text {wall }}=-2$ deg, $\left.\operatorname{Re}=1.01^{*} 10^{6}, \sigma=1.0\right)$, cavity contour and velocity vectors at mid-span at time $\mathrm{t} / \mathrm{T}=6.65$ (left) and $\mathrm{t} / \mathrm{T}=6.9$ (right)
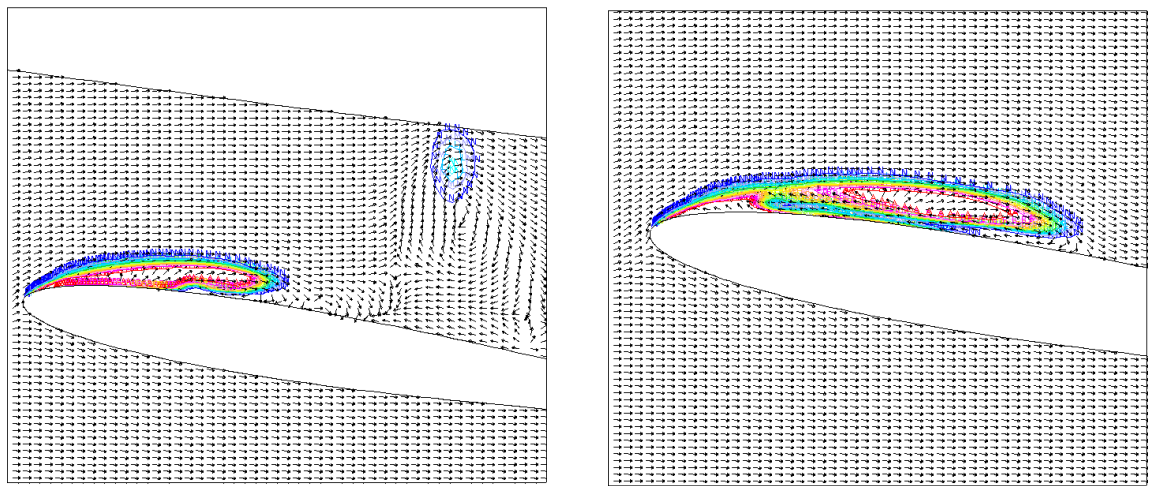

Figure 5.13: Twist 11 hydrofoil configuration $\left(\alpha_{\max }=11 \mathrm{deg}\right.$ and $\alpha_{\text {wall }}=-2$ deg, $\operatorname{Re}=1.01^{*} 10^{6}, \sigma=1.0$ ), cavity contour and velocity vectors at mid-span at time $\mathrm{t} / \mathrm{T}=7.28$ (left) and $\mathrm{t} / \mathrm{T}=7.56$ (right) 
The first drop in lift at $\mathrm{t} / \mathrm{T}=6.9$ is associated with the second picture in figure 5.10 and figure 5.12. It is the time the main cavity cloud detaches from the leading edge cavity. These cavities, with a strong vortical flow, collapse on the hydrofoil producing high pressure wave of up to $105 \mathrm{kPa}$ amplitude. The second drop in lift at $\mathrm{t} / \mathrm{T}=7.56$ is related to the main cloud collapse above the upper surface of the hydrofoil, see the last and fourth picture in figure 5.10 and 5.11, with an associated pressure peak of up to about $70 \mathrm{kPa}$ amplitude.

The time steps during which high pressure amplitudes are observed are associated with the occurrence of regions with high values of the vorticity and reduction in lift. This analysis shows that CFD is able to predict high pressure waves and identify regions with a high risk for cavitation erosion. These results are also in line with results of the numerical simulations presented in [74] using an Euler method for inviscid compressible flow. Still with the current method, the level of the peak pressures is not captured at the highest level found in [74], although in measurements (see [47]) variations in pressure from order $100 \mathrm{kPa}$ to $10 \mathrm{MPa}$ bar have been measured.

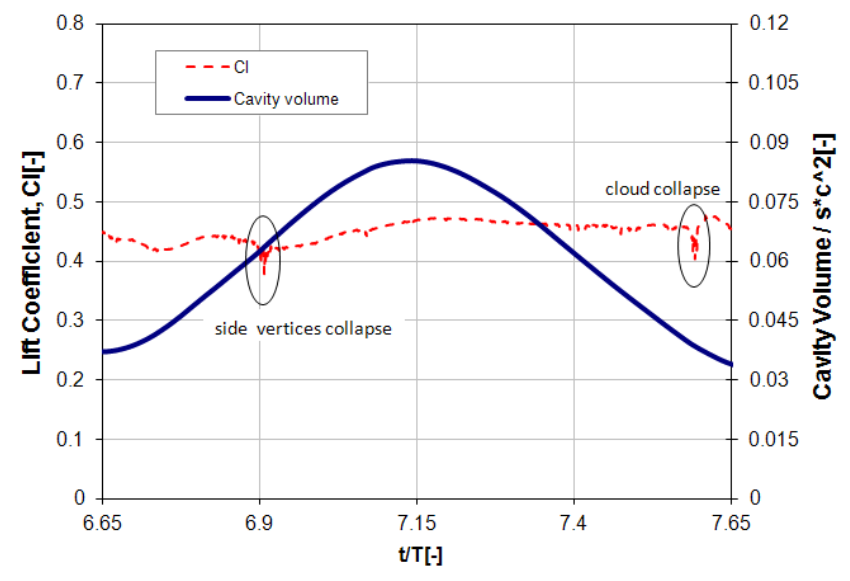

Figure 5.14: Twist 11 hydrofoil configuration $\left(\alpha_{\max }=11 \mathrm{deg}\right.$ and $\alpha_{\text {wall }}=-2$ $\left.\mathrm{deg}, \operatorname{Re}=1.01^{*} 10^{6}, \sigma=1.0\right)$. Variation of lift coefficient and cavity volume during one period 

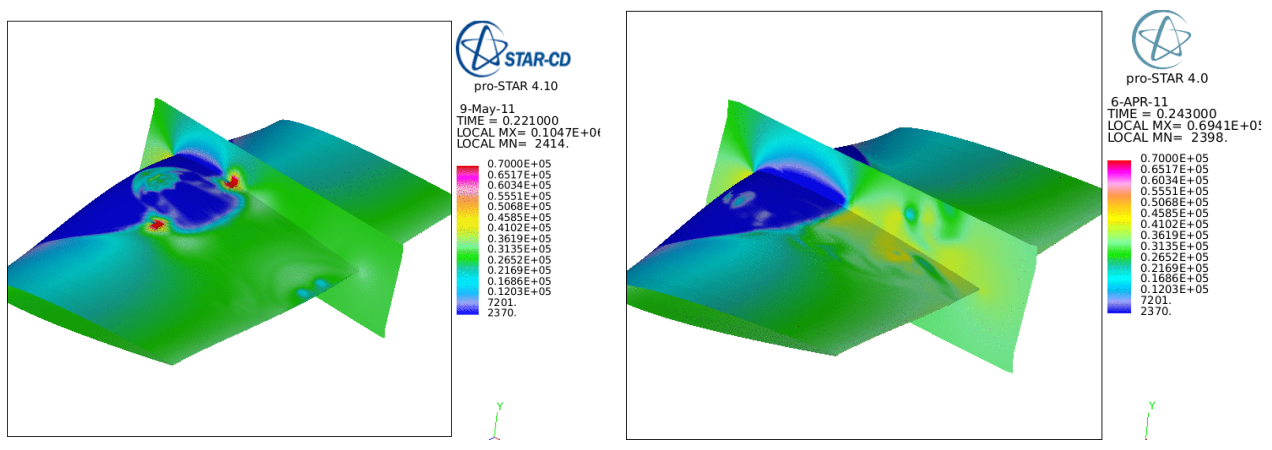

Figure 5.15: Twist 11 hydrofoil configuration $\left(\alpha_{\max }=11 \mathrm{deg}\right.$ and $\alpha_{\text {wall }}=-2$ deg, $\operatorname{Re}=1.01 * 10^{6}, \sigma=1.0$ ), wall pressure contours at $\mathrm{t} / \mathrm{T}=6.9$ (left) and $\mathrm{t} / \mathrm{T}=7.56$ (right)

\subsubsection{Conclusions}

The Twist 11 hydrofoil test case has been selected in the present study because it provides the most basic features of a shedding cavity: the shedding of the main leading edge cavity at a fixed frequency. Testing the numerical method on such a case gives important information on the capability of the method to predict the associated complex flow mechanisms.

The study of the hydro-dynamics of the Twist 11 hydrofoil reviled that the standard $k-\varepsilon$ and the RNG $k-\varepsilon$ turbulence models are not able to reproduce the cavity shedding observed in the experiments. This appears to happen mainly due to the inability of these models to reproduce the strong re-entrant jet that cuts off the main leading edge cavity into a cloud of vapor, because of an over prediction of turbulent viscosity at the liquid-vapor interface. The RNG $k-\varepsilon$ turbulence model with a density based modification of the eddy viscosity in the region of the liquid-vapor interface (as from [24]), is reducing the turbulent viscosity (apparent reduction of the turbulent viscosity at the vapor liquid interface). In the liquid-vapor mixture the velocity is increased resulting in a "strong" 3D jet and forming complex three dimensional cavity shapes. Thus for the $3 \mathrm{D}$ test case of the cavitating flow around the Twist 11 hydrofoil, the computational method used is able to reproduce cavity shedding on macro-structure level possibly indicating areas of erosion risk by means of high pressure waves associated with cloud collapse above the hydrofoil, see the study of [50].

Lacking from the numerical simulations of cavitation are the numerous small cavitating vortex structures, the micro-jets and the associated high pressure 
waves. The grid resolution and turbulence modeling are main causes of this fine structures missing from the numerical predictions. While the method for incompressible flow is not able to capture the associated high pressure amplitudes up to 10MPa (as found in [47]) and shock wakes (see [74]) are not captured.

\subsection{D Elliptic 11 rake hydrofoil}

\subsubsection{Geometry}

The Elliptic 11 Rake hydrofoil is a 3D hydrofoil with tip rake, in this case specially designed to develop a strong cavitating leading edge-vortex. The section of the hydrofoil is again the symmetric NACA 0009 section, see equation 4.22 , with a maximum thickness of $\mathrm{t} / \mathrm{c}=0.09$.

The chord distribution for the hydrofoil is an ellipse with a finite tip length.

$$
c(\bar{y})=\sqrt{C_{R}^{2}\left(1-\bar{y}^{2}\right)+\bar{y}^{2} C_{T}^{2}}
$$

for $\bar{y}=y /\left(\frac{b}{2}\right) \in[0,1]$ The chord at the tip is $C_{T}=50 \mathrm{~mm}$. The chord at the root is $C_{R}=150 \mathrm{~mm}$ and the span of the foil is $S=200 \mathrm{~mm}$. The angle of attack distribution is defined by:

$$
\alpha(\bar{y})=\alpha_{t} \bar{y}\left(\frac{e^{\bar{y}_{\alpha} \bar{y}}-1}{e^{\bar{y}_{\alpha}}-1}\right)+\beta
$$

for $\bar{y} \in[0,1], \alpha=11 \mathrm{deg}, \beta$ is the angle of attack of the entire hydrofoil which can vary from +3 to -3 degrees and $y_{\alpha}$ is a shape parameter set equal to 7 . Figure 5.16 shows the angle of attack distribution for $\beta=0$.

For rake a similar distribution is used as for the angle of attack:

$$
R(\bar{y})=R_{t} \bar{y}\left(\frac{e^{\gamma_{R} \bar{y}}-1}{e^{\gamma_{R}}-1}\right)
$$

for $\bar{y} \in[0,1], R_{t}=20 \mathrm{~mm}$ is the rake at the tip and the shape parameter $\gamma_{R}=1$. The skew of the ellipse is given by the equation:

$$
s(\bar{y})=C(\bar{y})-C_{R}
$$

For the configuration with the above described geometry, van der Hout [87] 


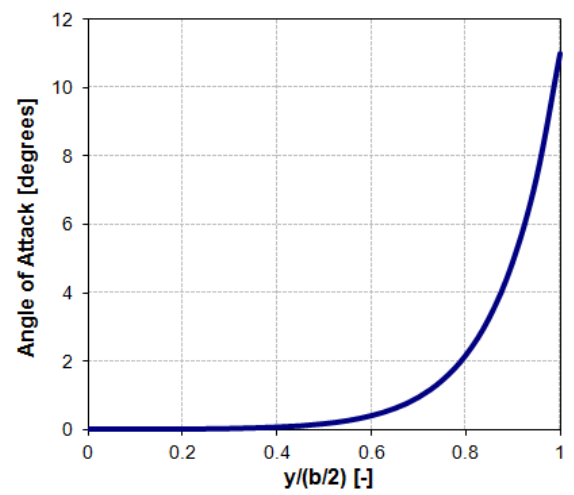

Figure 5.16: Angle of Attack, Elliptic 11 Rake foil

performed experiments in the cavitation tunnel at Delft University of Technology to investigate the interaction between sheet and vortex cavitation for steady and unsteady inflow conditions. In the present study only steady inflow conditions are considered and the experimental set-up is reproduced in the computational set-up. The results of the numerical simulations are compared with the measurements for wetted and cavitating flow.

\subsubsection{Computational domain and mesh}

The size of the computational domain is defined as follows: inlet location at 2 root chords upstream of leading edge at the root, outlet at 3 root chords downstream of trailing edge at the root and a test section of 2 by 2 root chords, as in the experimental setup, see figure 5.17.

The mesh for the wing is created with a structured multi-block hexahedral mesh generator. around the hydrofoil is a C-grid (due to the sharp trailing edge), in order to maintain control over the quality of the mesh near the hydrofoil. Development of the boundary layer along the hydrofoil surface is taken into account using wall functions. The requirement for the value of $y^{+}$ at the hydrofoil surface can be met with an acceptable number of cells in the normal direction. Moreover, the aspect ratio and the differences in cell sizes can be kept low. Figure 5.18 shows the surface mesh of the Elliptic 11 Rake hydrofoil.

The C-grid around the root section is shown in figure 5.19. This type of meshing is efficient for control of $y^{+}$, which is kept constant for all grid varia- 


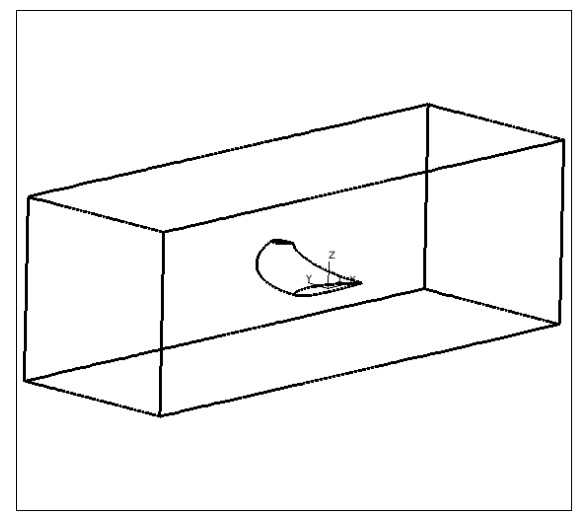

Figure 5.17: Computational Domain, Elliptic 11 Rake hydrofoil

tions, with values between 15 and 100, as recommended for the wall function approach [17].

Figure 5.17 shows the computational domain. The reference Cartesian coordinate system has the $\mathrm{x}$-axis in chord-wise (flow) direction, $\mathrm{y}$-axis in spanwise direction and the $\mathrm{z}$-axis is normal to the $\mathrm{x}$ and $\mathrm{y}$-direction.

A detailed numerical investigation has been carried out for the Elliptic 11 rake hydrofoil both in wetted flow and in a cavitating flow, for different grid resolutions in the tip vortex region. Also, based on the available data, predicted forces and velocity fields are compared with results from experiments. Note that the details about the analysed grids (G1, G2 and G3) are given in the Uncertainty Study chapter. For these grids surface grid guidelines are obeyed.

\subsubsection{Numerical parameters}

The boundary conditions applied are: an upstream inlet boundary condition, which requires the prescription of the velocity components and additional values for the turbulence intensity. At the domain sides, slip wall boundary conditions are imposed. At the downstream outlet, a constant pressure boundary condition is applied. All boundary conditions are summarized in table 5.5 . 


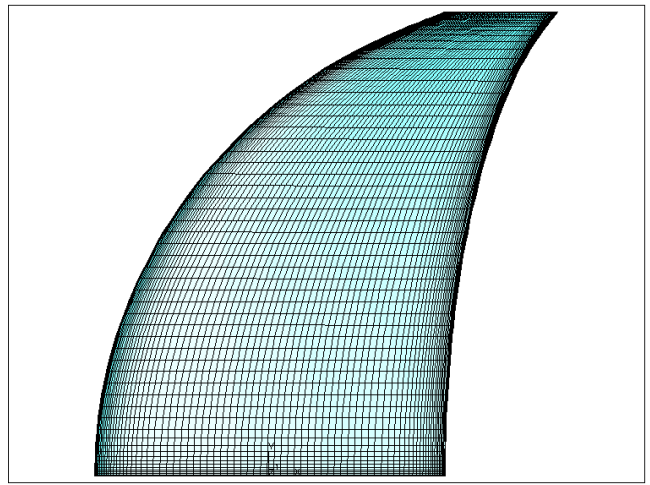

Figure 5.18: Surface mesh, Elliptic 11 Rake hydrofoil

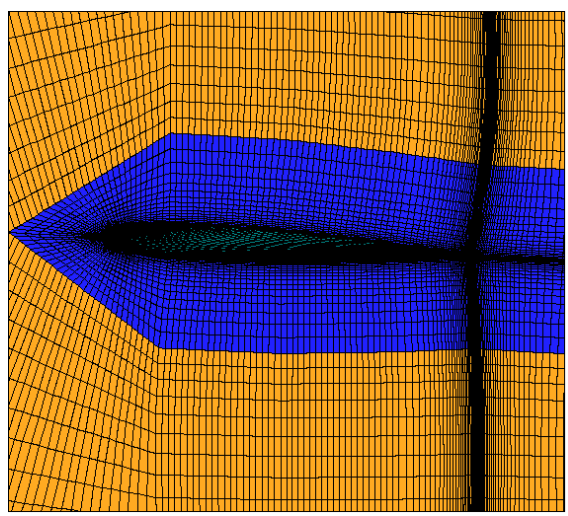

Figure 5.19: C-grid root section of Elliptic 11 Rake hydrofoil, Leading Edge (left) and Trailing Edge (right)

\begin{tabular}{|c|c|}
\hline Boundary Name / Location & Boundary Type \\
\hline Inlet & Inlet Velocity \\
\hline Sides & Slip Wall \\
\hline Outlet & Pressure \\
\hline Hydrofoil & No-slip Wall \\
\hline
\end{tabular}

Table 5.5: Boundary conditions, Elliptic 11 rake hydrofoil, angle $\beta=3 \mathrm{deg}$

The RANS equations are solved using the implicit SIMPLE algorithm and the spatial discretization is second-order accurate using the MARS scheme, 
as in the preceding cases. The turbulence model choice is the RNG $k-\varepsilon$ model and the RNG $k-\varepsilon$ modified model since from the simulations for the Twist 11 hydrofoil it proved to be the most appropriate turbulence model to predict wetted and cavitating flow in good agreement with results from measurements.

\subsubsection{Results wetted flow}

The Elliptic 11 Rake hydrofoil is first considered for the case of wetted flow for an inlet velocity of $7.43 \mathrm{~m} / \mathrm{s}$ and an outlet pressure of $21700 \mathrm{~Pa}$. Note that validation of the numerical results for wetted flow is not possible due to the absence of experimental data. In the following only numerical results are considered.

The effect of grid refinement (grids G1, G2 and G3) was assessed in the Uncertainty Study Chapter. In the following results obtained for the finest mesh (G3) and a fourth grid G4 (created by refining the tip vortex region of grid G3) are analysed to assess the prediction of the lift and drag coefficients. The tip vortex refinement is based on the method described in the Vortex Detection and Visualisation Chapter. Cells with a Q-factor higher than a certain threshold value are refined with a grid ratio of 2 in all directions. Details of grids G3 and G4 are found in table 5.6.

\begin{tabular}{|c|c|c|}
\hline Cells & G3 & G4 \\
\hline Total Number of Cells & $1.181 * 10^{6}$ & $1.852 * 10^{6}$ \\
\hline Section Vertices & 192 & 192 \\
\hline
\end{tabular}

Table 5.6: Details of meshes, Elliptic 11 rake hydrofoil, angle $\beta=3 \mathrm{deg}$

From comparing results for grid G3 and G4 the effect of the mesh refinement at the location of the tip vortex is evaluated.

The lift and drag coefficients are given in table 5.7.

\begin{tabular}{|c|c|c|}
\hline & $\mathrm{G} 3$ & $\mathrm{G} 4$ \\
\hline$C_{L}$ & 0.1266 & 0.1273 \\
\hline$C_{D}$ & 0.00872 & 0.00898 \\
\hline
\end{tabular}

Table 5.7: Lift and drag coefficients for different meshes for wetted flow condition, Elliptic 11 rake hydrofoil. $\beta=3 \mathrm{deg}, \mathrm{Re}=1.1^{*} 10^{6}$, RNG $k-\varepsilon$ turbulence model 
Table 5.7 shows that local grid refinement for resolving the tip vortex has limited effect on the lift and drag coefficient, $0.5 \%$ on lift and $2.8 \%$ on drag when comparing the values obtained for grid G3. The small differences are caused by the change in the surface pressure coefficient distribution, see figure 5.20 for the entire geometry; and figure 5.21 for a detail in the tip region.

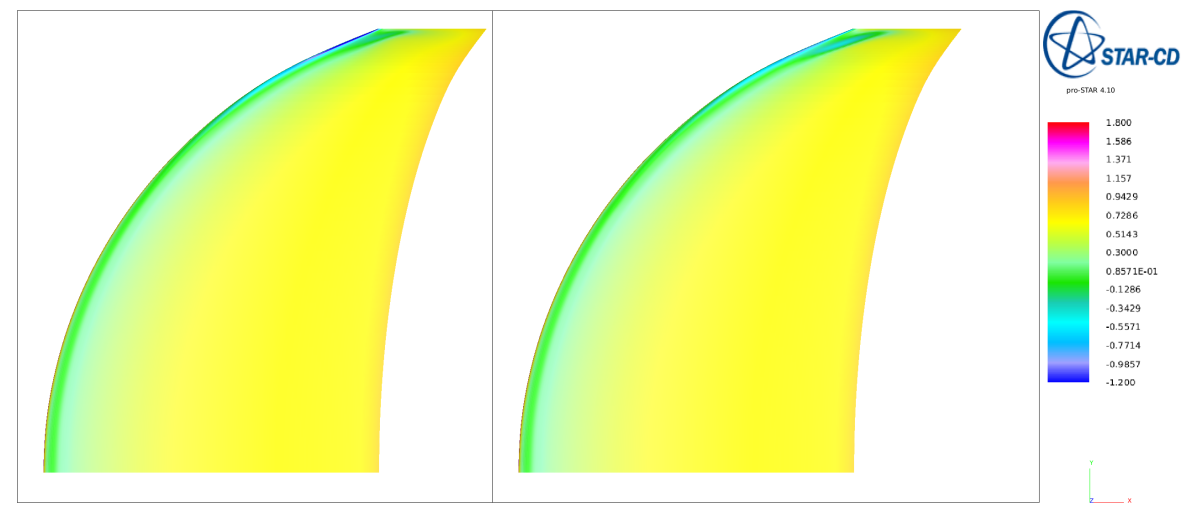

Figure 5.20: Elliptic 11 rake hydrofoil, angle of attack $\beta=3 \mathrm{deg}, \operatorname{Re}=1.1^{*} 10^{6}$, surface pressure distribution, grids G3 (left) and G4 (right)

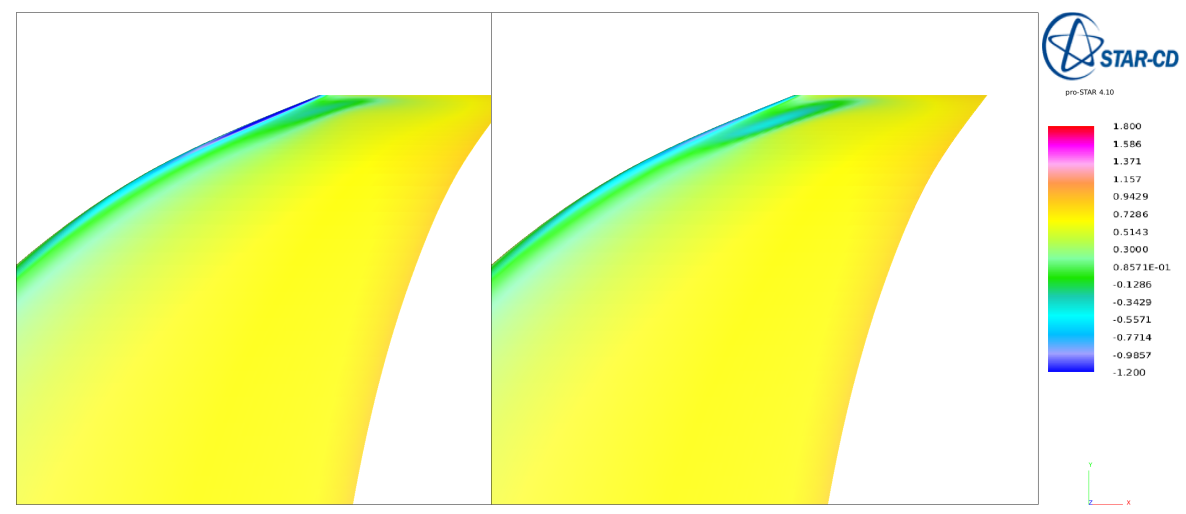

Figure 5.21: Elliptic 11 rake hydrofoil, angle of attack $\beta=3 \mathrm{deg}, \operatorname{Re}=1.1^{*} 10^{6}$, surface pressure distribution at tip, grids G3 (left) and G4 (right)

In the following, the solution in the tip vortex region is analysed, which is 
the main interest of this test case.

For topics like vortex definition, detection and visualization, the reader is referred to the Vortex Detection and Visualization Chapter. The Q factor criterion, previously defined as:

$$
Q=\frac{1}{2}\left[\left(\frac{\partial u_{i}}{\partial x_{i}}\right)^{2}-\frac{\partial u_{i}}{\partial x_{j}} \frac{\partial u_{j}}{\partial x_{i}}\right]
$$

is used to capture regions with a high value of the vorticity.

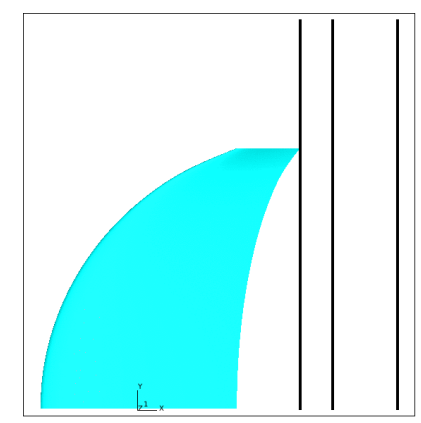

Figure 5.22: Downstream cross-flow planes at chord-wise locations $\mathrm{x}=0.125$, 0.15 and $0.2 \mathrm{~m}$, Elliptic 11 Rake foil, angle of attack $\beta=3 \mathrm{deg}$

Flow quantities are analysed downstream of the hydrofoil at stream-wise location $\mathrm{x}=0.15 \mathrm{~m}$ (see figure 5.22 ) which is the plane placed $15 \mathrm{~cm}$ downstream of the mid-chord location of the root section. The pressure distribution on surface of the hydrofoil is presented in figures 5.20 and 5.21 for grid G3 (left) and grid G4 (right), respectively.

The surface pressure distribution is only slightly affected by the mesh refinement in grid G4. Only near the tip it appears that in the leading edge region the low pressure region below the leading edge vortex is resolved better. Figure 5.23 presents the pressure distribution in the plane $\mathrm{x}=0.15$ downstream of the hydrofoil along a traverse through the vortex core.

As expected, for an increasing mesh density, the minimum pressure within the tip vortex core decreases. Overall mesh refinement (grids G1, G2 and G3; as studied in the Uncertainty Study Chapter) slightly improves the pressure distribution. But, introducing mesh G4, with a refined mesh in the region of the vortex core, resolves the pressure distribution better in terms of low pressure within the tip vortex core. 
The distribution of the $\mathrm{W}$-component of the velocity (in vertical direction) along the traverse through the vortex core (figure 5.24) is improved also slightly by refining the grid in a global manner. The minimum and maximum values of this azimuthal velocity component are further improved when local mesh refinement is applied (grid G4). The slope of the distribution increases considerably and with that the axial component of the velocity in the vortex core.

Accurate predictions of the velocity components in the region of the tip vortex are important in the determination of the vortex location by means of the Q-factor criterion. Therefore flow feature refined grids are a must for the accuracy of this kind of numerical simulations.

From the Q-factor criterion, it is known that a vortex is defined as a region in the flow in which the Q-factor is positive. In figure 5.25 the high peaks in the Q-factor indicate a vortex region, as already observed from the distribution of the pressure and the velocity components. Local vortex refinement applied to grid G3 results in a Q-factor of 106000 for G4 instead of 36000 for grid G3. This indicates again that the vortex is better resolved on the finer mesh and that the Q-factor is, similar to $C_{p}$, sensitive to changes in local grid density.

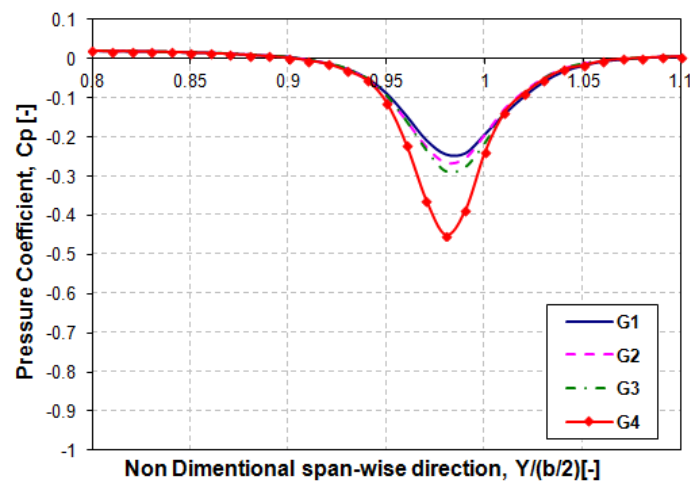

Figure 5.23: Elliptic 11 rake hydrofoil, angle of attack $\beta=3 \mathrm{deg}, \operatorname{Re}=1.1^{*} 10^{6}$, Cp distribution along traverse through vortex core for grids G1, G2, G3 and G4, wetted flow, cross-flow plane at $\mathrm{x}=0.15 \mathrm{~m}$

Downstream of the Elliptic 11 rake hydrofoil, the tip vortex decays fast as evident from figures 5.26 through 5.28 for $\mathrm{x}=0.125 \mathrm{~m}, 0.15 \mathrm{~m}$ and $0.2 \mathrm{~m}$, respectively. Further refinement may be needed to capture the characteristics 


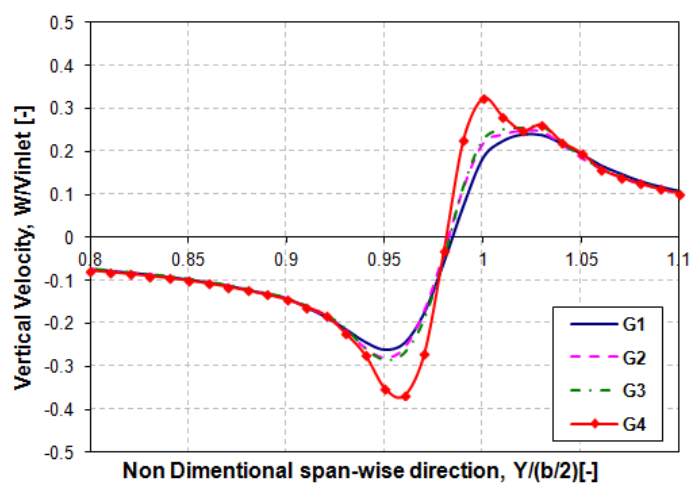

Figure 5.24: Elliptic 11 rake hydrofoil, angle of attack $\beta=3 \mathrm{deg}, \operatorname{Re}=1.1^{*} 10^{6}$, at $\mathrm{x}=0.15 \mathrm{~m}$, non-dimensional normal velocity $(\mathrm{W})$ along traverse through vortex core for grids G1, G2, G3 and G4, wetted flow

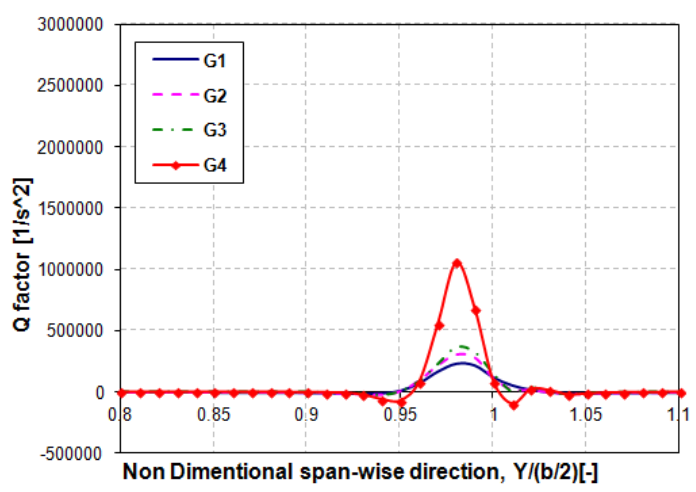

Figure 5.25: Elliptic 11 rake hydrofoil, angle of attack $\beta=3 \mathrm{deg}, \operatorname{Re}=1.1^{*} 10^{6}$, traverse through vortex core in cross-flow plane at $\mathrm{x}=0.15 \mathrm{~m}$, distribution $\mathrm{Q}$ factor for grids G1, G2, G3 and G4, wetted flow

of vortices in terms of low pressure in the core and high values of the Q-factor. Being limited to grids containing about 1.5 to 2 million cells (a second tip vortex refinement will increase the grid to about 5 to 6 million cells since the vortex region is already fine) the current mesh is adequate to capture the low pressure inside the leading edge-tip vortex and to locate the tip vortex by the Q-factor criterion. Anticipating the requirement for the numerical simulations for propellers, the present hydrofoil geometry has to provide guidelines 
in terms of meshing.

Thus, from the results for the wetted flow around the Elliptic 11 rake hydrofoil it can be concluded that when dealing with tip vortices local refinement is crucial for an accurate prediction of the pressure and velocity distribution inside their core.

It follows from figure 5.26, 5.27 and 5.28 that the stream-wise development of the tip vortex core is characterised by a lowering of the slope of the azimuthal velocity as function of the span-wise coordinate. This implies a spreading of the vortex core, i.e. vortex core becomes less compact. The maximum of the axial wake of the vorticity decreases and the minimum pressure increases. However, the circulation of the vortex core does not change very much. In the numerics, the axial development of the vortex core is much faster than in reality for which the vortex core remains over a much longer distance compact.

The present investigation of the wetted flow condition is aimed at detecting the tip vortex and the corresponding region with low pressure. In the next section cavitating flow is analysed for which validation data is available from experiments $([87])$.

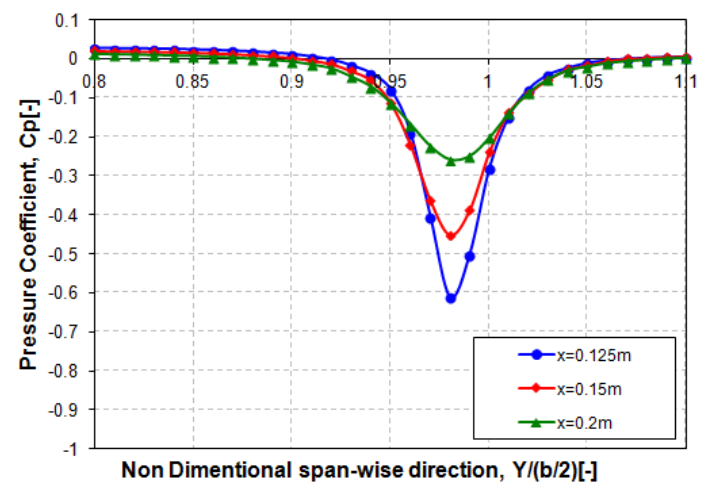

Figure 5.26: Cp distribution along traverse through tip vortex core in a number of cross-flow planes downstream of Elliptic 11 rake hydrofoil, angle of attack $\beta=3 \mathrm{deg}, \operatorname{Re}=1.1^{*} 10^{6}$, wetted flow, grid G4 


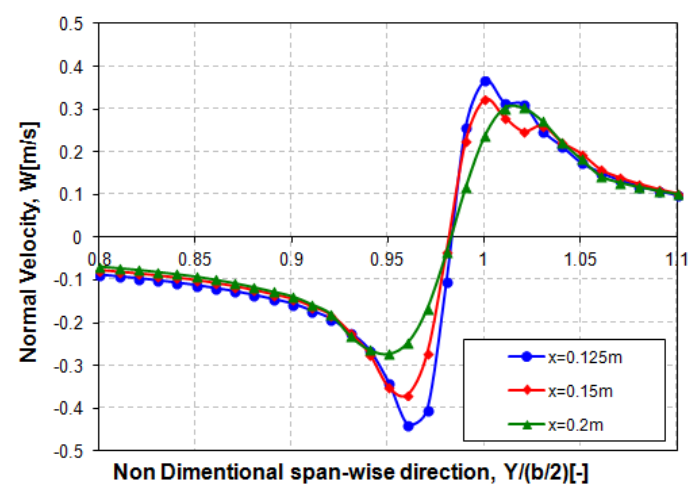

Figure 5.27: Distribution of vertical velocity (W) along a number of traverses through tip vortex core in cross-flow planes downstream of Elliptic 11 rake, angle of attack $\beta=3 \mathrm{deg}, \operatorname{Re}=1.1 * 10^{6}$, wetted flow, grid G4

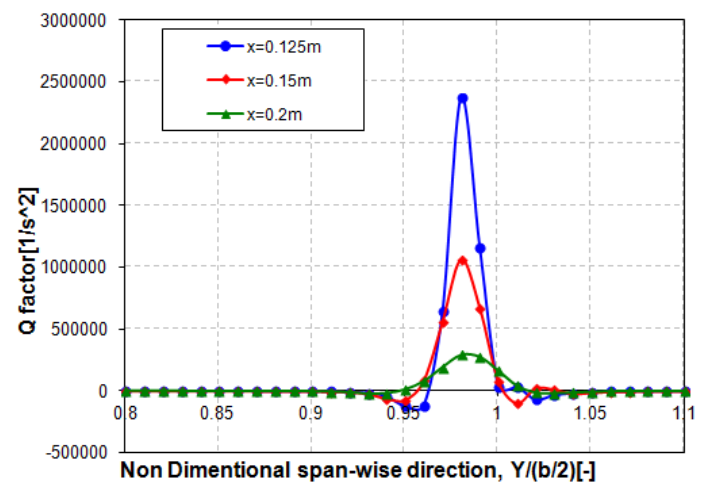

Figure 5.28: Q-factor distribution along traverse through the tip vortex core in a number of cross-flow planes downstream of Elliptic 11 rake hydrofoil, angle of attack $\beta=3 \mathrm{deg}, \operatorname{Re}=1.1^{*} 10^{6}$, wetted flow, grid $\mathrm{G} 4$

\subsubsection{Results cavitating flow, $\sigma=0.68$}

When the ambient pressure is reduced, cavitation will occur in the flow over the Elliptic 11 Rake hydrofoil. All parameters like: lift, drag, distribution of pressure and velocity are affected. In the following, the results obtained for a cavitation number of $\sigma=0.68$, are presented. The numerical results are validated with available experimental results of [87]. 

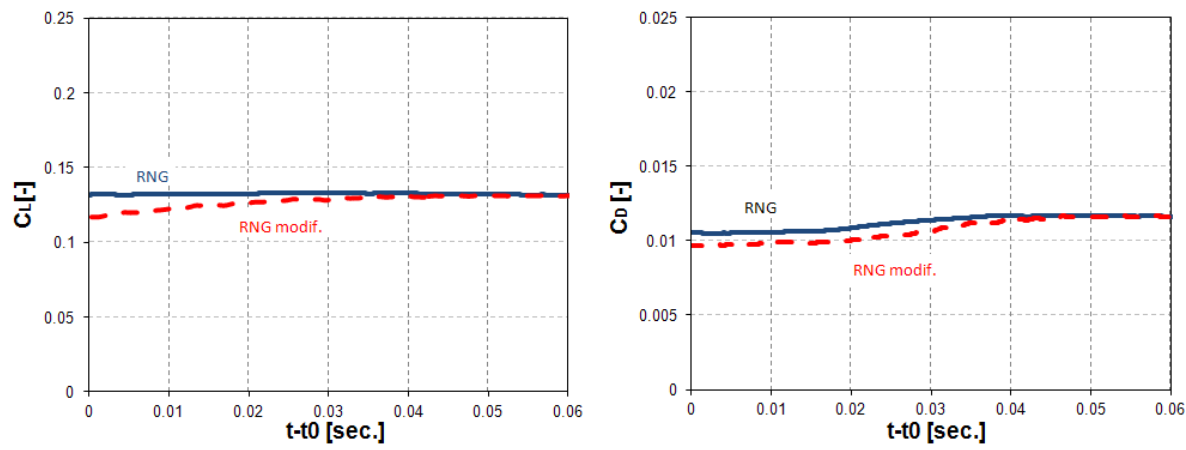

Figure 5.29: Elliptic 11 rake hydrofoil, angle of attack $\beta=3 \mathrm{deg}$, grid G4, $\mathrm{Re}=1.1^{*} 10^{6}$, convergence of lift (left) and drag (right) coefficients, cavitating case $\sigma=0.68$

Note that for this case of cavitating flow the RNG $k-\varepsilon$ and the RNG $k-\varepsilon$ modified turbulence models are used. In the present case of sheet-tip vortex cavitation, lift and drag are used to monitor the convergence of the solution, since in the present case the cavitation volume is more or less constant and not periodical. Figure 5.29 show the results for the convergence of the lift and drag coefficients of the Elliptic 11 rake hydrofoil in cavitating conditions, using the RNG $k-\varepsilon$ (continuous blue lines) and RNG $k-\varepsilon$ modified (dashed red lines) turbulence models.

The converged values of lift and drag coefficients for cavitating flow (as seen from figure 5.29) are presented in table 5.8 and compared with the lift and drag coefficients, in case of wetted flow. Compared to wetted flow, for cavitating flow the lift and drag coefficients are higher. For the RNG $k-\varepsilon$ turbulence model this is $2.97 \%$ for the lift and $22.67 \%$ for the drag coefficient, while for the RNG $k-\varepsilon$ modified turbulence model this is $2.67 \%$ for the lift and $22.68 \%$ for the drag coefficient. These differences between the two results for cavitating flow but for different turbulence model are considered to be within the numerical uncertainty.

Lift and drag coefficients are compared with corresponding experimental data in table 5.8 for the cavitating flow at $\sigma=0.68$. The lift coefficient is in good agreement with measurements for both turbulence models $(1.26 \%$ for RNG and $1 \%$ for RNG modified). The drag coefficient is over-predicted for both turbulence models ( $12.27 \%$ for RNG and $12.28 \%$ for RNG modified). For the Elliptic 11 rake hydrofoil, the two turbulence models tested have a limited influence on the predicted force coefficients. 


\begin{tabular}{|c|c|c|c|c|}
\hline & Wetted & $\sigma=0.68, \mathrm{RNG}$ & $\sigma=0.68$, RNG modified & EXP, $\sigma=0.68$ \\
\hline$C_{L}$ & 0.1274 & 0.1313 & 0.1309 & 0.1297 \\
\hline$C_{D}$ & 0.00898 & 0.011616 & 0.011615 & 0.00935 \\
\hline
\end{tabular}

Table 5.8: Elliptic 11 rake hydrofoil, angle of attack $\beta=3 \mathrm{deg}$, grid G4, $\operatorname{Re}=1.1 * 10^{6}$, Predicted lift and drag coefficients for wetted flow and for cavitating flow. Comparison of CFD data and experimental data

As for the wetted flow case, the analysis of the tip vortex is of main interest for the present test case of cavitating flow. The pressure distribution along a traverse through the core of the tip vortex in the cross-flow plane at $\mathrm{x}=0.15 \mathrm{~m}$ (see figure 5.22) for the wetted flow and the cavitating flow is presented in figure 5.30. The surface pressure distribution, together with the iso-pressure surface $C_{p}=-0.68$ are shown in figure 5.31 for wetted and cavitating flow. Figure 5.30 shows that the pressure coefficient decreases due to the occurrence of cavitation within the vortex core. This is usually seen when the cavitation number is chosen lower than the minimum pressure in wetted flow. In these cases the extent of the cavity is larger than the wetted flow indicates in terms of region with $C_{p}<-\sigma$. The minimum pressure coefficient is around -0.68 in agreement with the cavitation number $\sigma$, i.e. $C_{p}=-\sigma$. Also, from corresponding figure 5.31 it is seen that the extent of the region with $C_{p}=-\sigma$ is larger for the cavitating flow than for the wetted flow.

This strengthening of the vortex core is partly due to the higher lift that is generated by the hydrofoil in cavitating flow, which increases the circulation of the tip vortex. This will be the effect of sheet cavitation which affects the surface pressure distribution, primarily increasing the region with low pressure on the suction side of the hydrofoil.

The distribution of the azimuthal (vertical) velocity (W), along a (horizontal) traverse through the vortex core is analysed in figure 5.32. It also shows an increase in the magnitude of the circulation and of the azimuthal velocity when cavitation occurs, which corresponds to the lower pressure. In figure 5.33 the contour plots of the vertical velocity in the cross-flow plane at $\mathrm{x}=0.15$ are presented. They show the difference in vortex formation at the tip of the foil in wetted and cavitating flow, respectively.

Figure 5.34 shows the distribution of the pressure and the vapor fraction along a traverse through the vortex core in three consecutive cross-flow planes. It shows that downstream of the $\mathrm{x}=0.15$ plane, the pressure coefficient in the core is higher than $C_{p}=-\sigma$ so that also the vapor fraction becomes zero. This implies that capturing cavitating vortices downstream of the Elliptic 11 rake foil, even a finer local (tip vortex region) mesh is required than used now. The current grid is sufficiently fine for the adequate 
prediction of the forces and tip vortex formation above the blade and in the close proximity of the tip.

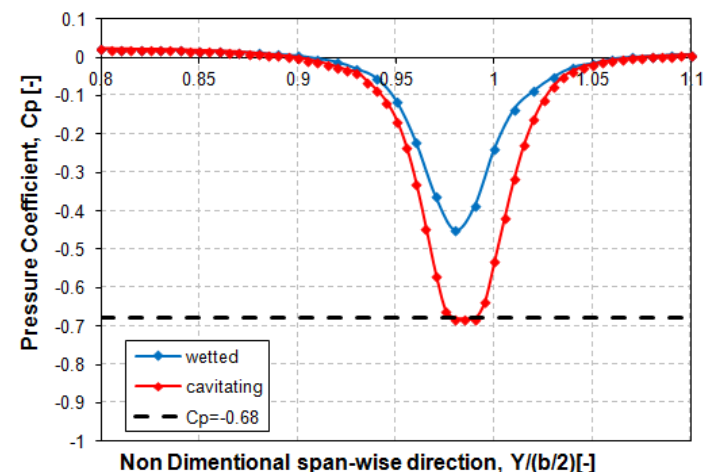

Figure 5.30: Elliptic 11 rake hydrofoil, angle of attack $\beta=3 \mathrm{deg}$, grid G4, $\mathrm{Re}=1.1^{*} 10^{6}$, grid G4, Pressure distribution along traverse through vortex core in plane at $\mathrm{x}=0.15 \mathrm{~m}, \sigma=0.68$

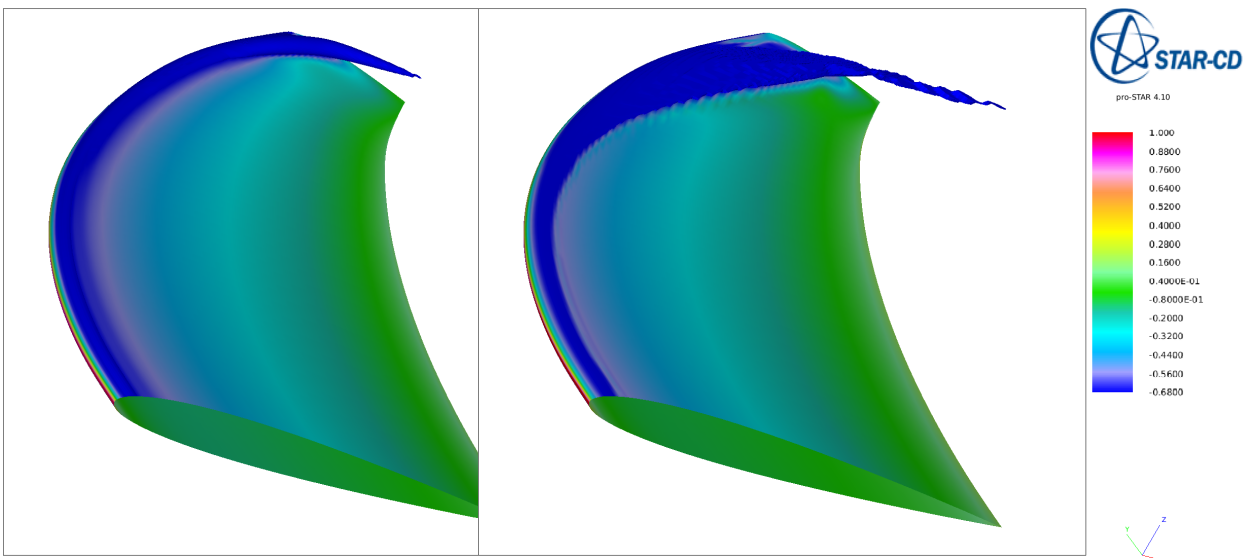

Figure 5.31: Elliptic 11 rake, angle of attach $\beta=3 \mathrm{deg}, \mathrm{Re}=1.1^{*} 10^{6}$, grid $\mathrm{G} 4$, $C_{p}$ distribution on surface hydrofoil and iso-surface $\mathrm{Cp}=-0.68$, wetted and cavitating flow $(\sigma=0.68)$ 


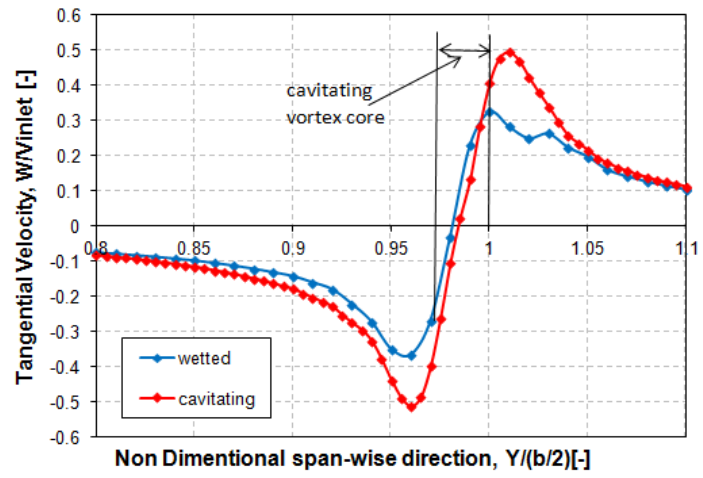

Figure 5.32: Elliptic 11 rake, angle of attack $\beta=3 \mathrm{deg}, \mathrm{Re}=1.1^{*} 10^{6}$, grid G4, Influence of cavitation on distribution of vertical (azimuthal) velocity $(\mathrm{W})$ along traverse through vortex core in plane $\mathrm{x}=0.15 \mathrm{~m}$

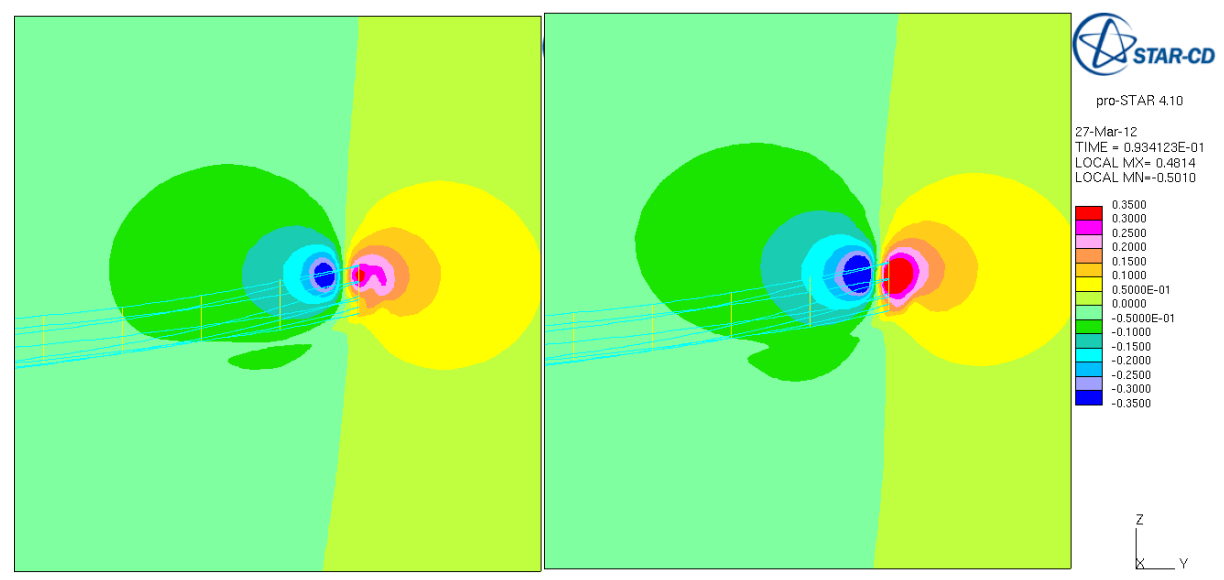

Figure 5.33: Elliptic 11 rake, angle of attack $\beta=3 \mathrm{deg}, \mathrm{Re}=1.1^{*} 10^{6}$, grid $\mathrm{G} 4$, distribution of vertical velocity $(\mathrm{W})$ in cross-flow plane $\mathrm{x}=0.15 \mathrm{~m}$, wetted (left) and cavitating flow (right)

The purpose of the current test case is the prediction of the formation and extent of the leading edge - tip vortex cavitation, as observed in the measurements of the flow around Elliptic 11 rake hydrofoil. In figure 5.35 the visualization results for the Elliptic 11 rake hydrofoil from [87] are compared with the results of the numerical simulations for the angle of attack $\beta=3 \mathrm{deg}$, 


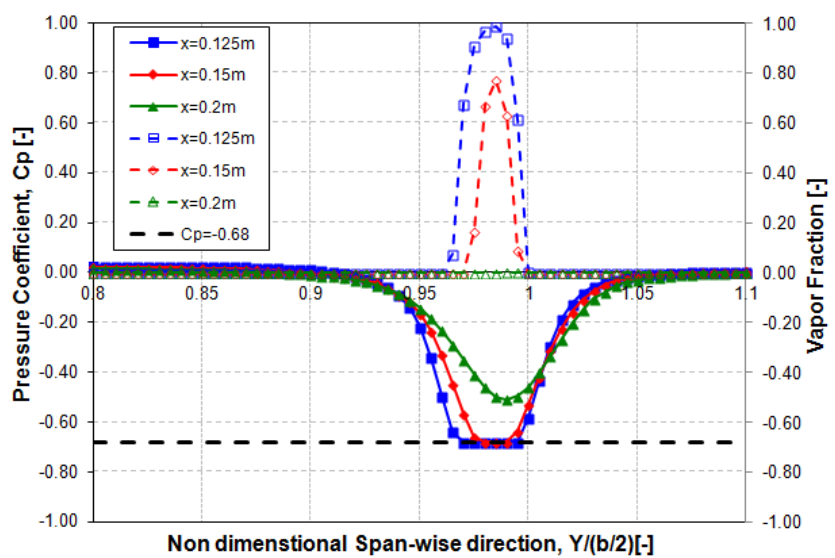

Figure 5.34: Elliptic 11 rake, angle of attack $\beta=3 \mathrm{deg}, \operatorname{Re}=1.1^{*} 10^{6}$, grid G4, Stream-wise development distribution of pressure and vapor fraction along traverse through core of vortex in a number of cross-flow planes, cavitating flow, $\sigma=0.68$

$\sigma=0.68$ and $\operatorname{Re}=1.1^{*} 10^{6}$.

Figure 5.35 shows the visualization of the cavitating flow as observed in experiments (left) and computations (right) as an iso-surface of the vapor fraction. for a volume fraction iso-surface of $1 \%$. The predicted cavity shape is similar to the one observed experimentally. The starting point of the reentrant jet and of the tip vortex as well as the detachment, as deduced from particle tracks, is in good qualitative agreement with the experimental observations (see figure 5.35). It is noted that choosing a different value for the vapor fraction for the cavity surface has limited influence on the cavity shape. Only small variations of the volume were noticed.

In order to determine the core of the tip vortex, the extent of the cavity that defines the vortex core has to be addressed. Analysing the iso-surface $C_{p}=-0.68$ and various vapor fraction iso-surfaces it has been found that the iso-surface vapor fraction $=0.3$ ( $30 \%$ vapor content) shows a good correspondence to the iso-surface $C_{p}=-0.68$, see figure 5.36.

Therefore, the vortex core diameter based on the iso-surface vapor fraction $=0.3$ is determined in various cross-flow planes, see figure 5.37. For these planes, the diameter of the tip vortex has been determined and plotted in figure 5.38. From the first four planes (chord-wise locations $\mathrm{x}=0.1$, $0.11,0.12$ and 0.13 ) the roll-up of the vortex layer from the hytdrofoil tip 


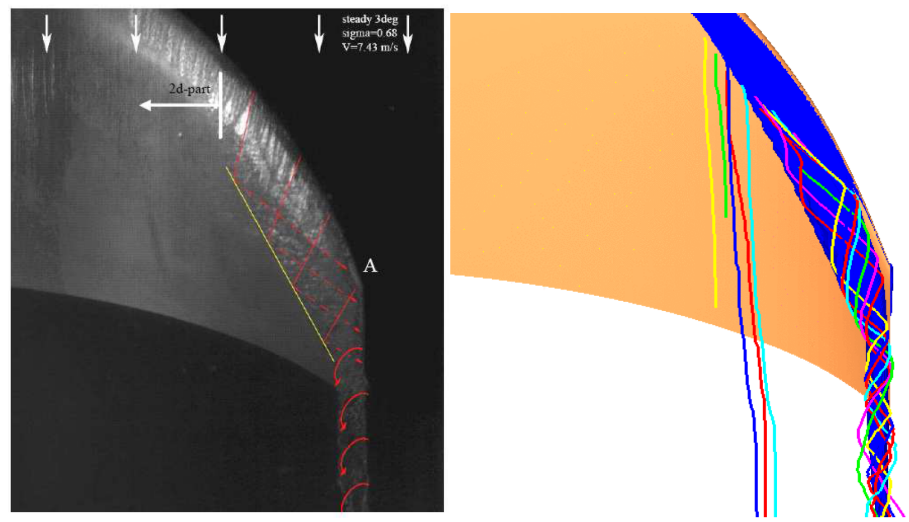

Figure 5.35: Elliptic 11 rake hydrofoil at $\sigma=0.68$, angle of attack $\beta=3 \mathrm{deg}$, $\mathrm{Re}=1.1^{*} 10^{6}$, Visualisation of the cavitating flow taken from [87] (left) versus result CFD simulation shown as the iso-surface vapor fraction $=0.01$ as well as the particle tracks (right)

and the trailing edge is still in process while further downstream the vortex decays rapidly. The vortex detaches from the foil tip in plane $\mathrm{x}=0.1 \mathrm{~m}$ to plane $\mathrm{x}=0.13 \mathrm{~m}$. In this region the maximum core diameter is found. It has an averaged value of about $6 \mathrm{~mm}$ (see 5.38) for the first five analysed planes. From experiments reported in [87] the vortex core is determined at a location corresponding to the plane $\mathrm{x}=0.13 \mathrm{~m}$ where it has an averaged value of about $10 \mathrm{~mm}$. Thus the predicted tip vortex core (based on the iso-surface vapor fraction $=0.3$ ) is more slender than the vortex in the experiment. This can be explained by the numerical simulations not resolving the small cavity structures. Also for the determinatiion of the size of the vortex core a lower vapor fraction iso-surface might have been considered. The last three planes analysed show a very fast decay of the cavitating tip vortex. This can have two reasons, one is due decreasing grid resolution in downstream direction and second is due to the dissipation introduced by the turbulence model and numerical scheme.

For the Elliptic 11 rake hydrofoil, the developed steady leading edge-tip vortex cavity is reproduced employing the standard RNG $k-\varepsilon$ turbulence model as well as when using the RNG $k-\varepsilon$ turbulence model with the modified density. The cavity is a steady leading-edge cavity developing into a tip vortex at the tip of the hydrofoil. Therefore in the current case the eddy viscosity in the vapor region interface jets are not playing an important role, thus both turbulence models (RNG $k-\varepsilon$ turbulence model and RNG $k-\varepsilon$ 
turbulence model modified) produce similar results.

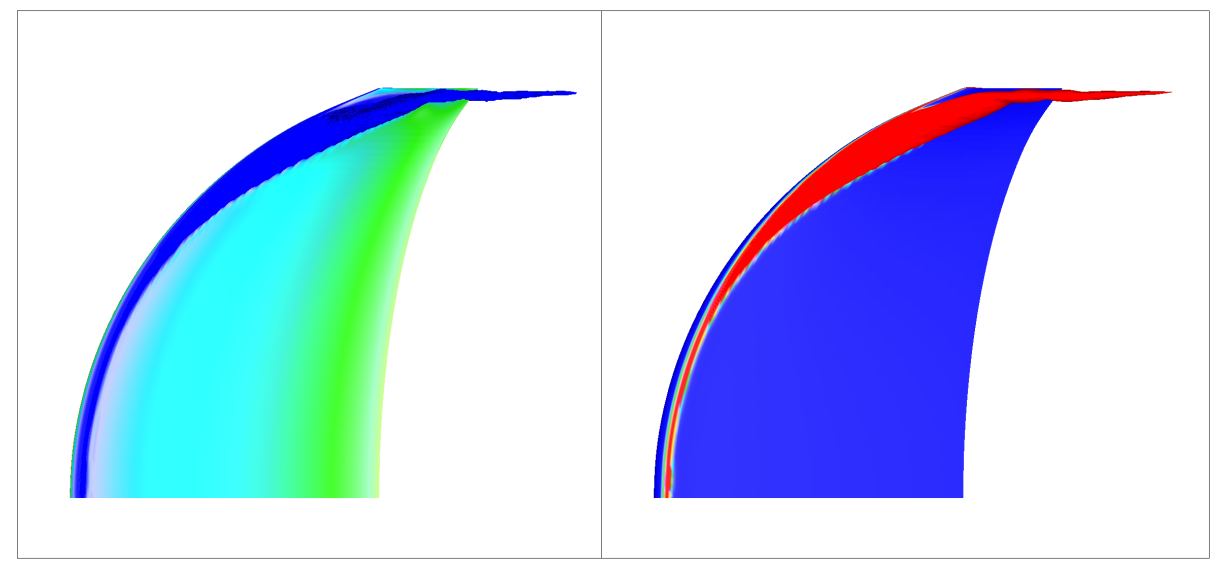

Figure 5.36: Elliptic 11 rake hydrofoil, angle of attack $\beta=3 \mathrm{deg}, \operatorname{Re}=1.1^{*} 10^{6}$, $\sigma=0.68$, grid G4. Surface pressure distribution and iso-surface $C_{p}=-0.68$ (left) and iso-surface vapor fraction $=0.3$ (right)

\subsubsection{Conclusions}

The Elliptic 11 rake hydrofoil is an especially designed geometry that develops a strong swirling flow in the tip vortex. Furthermore, this hydrofoil exhibits a flow that transitions from a mainly 2D sheet cavity in the inboard part of the hydrofoil to the tip vortex cavity in the outboard part of the hydrofoil. This pattern of cavities is representative for the cavitation seen on many propellers. These basic features of the leading edge-tip vortex are predicted by the present method and observed in experiments, in both wetted and cavitating flow conditions.

For the leading edge-tip vortex cavitation test case, both the RNG and the RNG modified turbulence models have been employed. It has been demonstrated that for the Elliptic 11 rake hydrofoil the standard and modified density approach within the mixture layer works equally well. This is the case because, the tip vortex formation is mainly due to side entrant jets from 


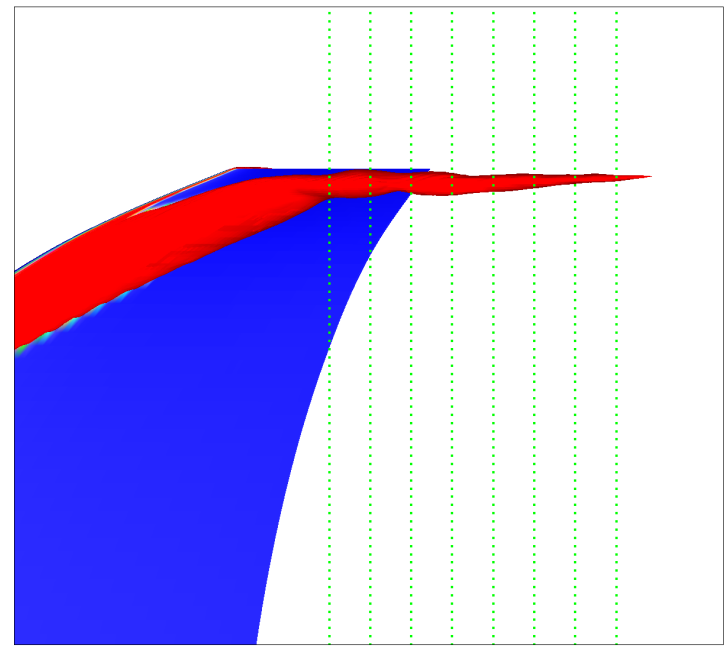

Figure 5.37: Elliptic 11 rake hydrofoil, angle of attack $\beta=3 \mathrm{deg}, \operatorname{Re}=1.1^{*} 10^{6}$, $\sigma=0.68$, grid G4, iso-surface vapor fraction $=0.3$. Also shown are the location of the cross-flow planes $\mathrm{x}=0.1,0.11,0.12,0.13,0.14,0.15,0.16$ and $0.17 \mathrm{~m}$

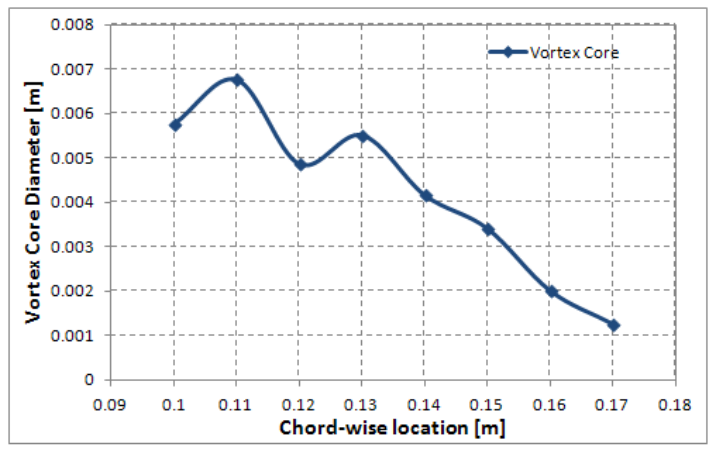

Figure 5.38: Elliptic 11 rake hydrofoil, angle of attack $\beta=3 \mathrm{deg}, \operatorname{Re}=1.1^{*} 10^{6}$, $\sigma=0.68$, grid G4, Diameter of vortex core as determined from predicted vapor fraction iso-contour $=0.3$

the main leading edge sheet cavity, that is fully cavitating, thus vortices produced in the turbulent mixing layer are not significant and the production of the turbulent viscosity is not affecting the results. 
While the method quantitatively predicts the cavitation patterns observed on the Elliptic 11 rake foil and the exhibited tip vortex cavitation, the numerical simulations are not able to capture the cavitating tip vortex far downstream, from about $0.15 \mathrm{~m}$ (about 1 chord) behind the chord at the tip. It is hypothesized that this is because the mesh resolution is not sufficiently fine but also due to the diffusive character of the two equation turbulence models which results in dissipation of the vortex features. In order to capture interaction effects of the tip vortex at far downstream locations an adequate mesh and a more advanced turbulence modeling (like RSM, LES and DES approaches, see [51] and [34]) are needed to improve the representation of the tip vortex.

\subsection{Conclusions on underlying flow mecha- nisms}

In the present Chapter, the capability of the numerical simulation to predict cavitating flows for two benchmark test cases (Twist 11 hydrofoil and Elliptic 11 rake hydrofoil) has been assessed.

These test cases are representative for: a complex 3D leading edge cavity shedding clouds (Twist 11 hydrofoil) and a leading edge-tip vortex (Elliptic 11 rake hydrofoil). The choice of the turbulence model and the effects of the resolution of the tip vortex in the grid have been investigated. As a general conclusion and guideline, the RNG $k-\varepsilon$ modified turbulence model in combination with a locally refined mesh in the tip vortex region appears to give sufficiently accurate results from the numerical simulation of these cavitating flows. It is clear from the present analysis that the choice of an appropriate turbulence model is essential for shedding cavities. This, in combination with meeting the requirements on the grid in order to achieve grid convergence and on the time step to achieve temporal convergence (as from the Uncertainty Study chapter).

For the Twist 11 hydrofoil and the Elliptic 11 rake hydrofoil cavitating flow cases, the performance of the RNG $k-\varepsilon$ turbulence model and the RNG $k-\varepsilon$ modified model has been investigated. The shedding of the $3 \mathrm{D}$ cavitating flow (Twist 11 hydrofoil) is predicted accurately pnly for the RNG $k-\varepsilon$ turbulence model with a correction for the turbulence viscosity within the mixture layer at the liquid-vapor interface. The leading edge-tip vortex cavitation (Elliptic 11 rake hydrofoil) is predicted using both turbulence models since in this case the mixture jets are do not play a major role. 


\begin{tabular}{|c|c|c|}
\hline Turbulence Model & Twist 11 hydrofoil & Elliptic 11 rake hydrofoil \\
\hline RNG $k-\varepsilon$ & invalid & valid \\
\hline RNG $k-\varepsilon$ modified & valid & valid \\
\hline
\end{tabular}

Table 5.9: validity turbulence models employed for cavitating flows for: Twist 11 hydrofoil and Elliptic 11 rake hydrofoil

In table 5.9 the validity of the turbulence models employed in the numerical simulations for the two test cases is summarized. The only turbulence model valid for all test cases is the RNG $k-\varepsilon$ turbulence model with correction for turbulent viscosity. This turbulence model is therefore applied in the numerical simulations dealing with propeller cavitation.

Moreover, based of underlying flow characteristics like:

- steady sheet cavity

- shedding sheet cavity and shedding frequency

- leading edge and tip vortex cavity

- interaction between sheet and vortex cavity: with and without shedding

- tip vortex core: close to the foil and far downstream

the proposed method (RANS equation, RNG modified turbulence model and the Sauer cavitation model) performs as follows from tables 5.10 and 5.11 for Twist 11 hydrofoil and Elliptic 11 rake hydrofoil, respectively.

\begin{tabular}{|l|c|}
\hline $\begin{array}{l}\text { Underlying flow characteris- } \\
\text { tics }\end{array}$ & Twist 11 hydrofoil \\
\hline steady sheet cavity & not simulated \\
\hline shedding sheet cavity & predicted \\
\hline shedding frequency & predicted \\
\hline
\end{tabular}

Table 5.10: Capability RANS approach to predict underlying flow characteristics of cavitating flow about Twist 11 hydrofoil

Note that other turbulence models like the $k-\omega$ or the $k-\omega$ SST are recommended mainly with the use of low $y^{+}$values and not especially suitable for far field flows (the SST model is actually the $k-\omega$ turbulence model that switches to the $k-\varepsilon$ model in the far field) like the tip vortex development in downstream direction, see [55] and [56]. The most appropriate 


\begin{tabular}{|l|c|}
\hline $\begin{array}{l}\text { Underlying flow characteris- } \\
\text { tics }\end{array}$ & Elliptic 11 rake hydrofoil \\
\hline leading edge and tip vortex cavity & predicted \\
\hline $\begin{array}{l}\text { interaction between sheet and } \\
\text { vortex cavity without shedding }\end{array}$ & predicted \\
\hline $\begin{array}{l}\text { interaction between sheet and } \\
\text { vortex cavity with shedding }\end{array}$ & not simulated \\
\hline tip vortex core within one chord & predicted \\
\hline tip vortex core far downstream & not predicted \\
\hline
\end{tabular}

Table 5.11: Capability RANS approach to predict underlying flow characteristics of cavitating flow about Elliptic 11 rake hydrofoil

turbulence model to be used with the wall functions approach is then the $k-\varepsilon$ turbulence model approach. Thus it is decided not to use the $k-\omega$ approach for the current cases in which the main interest is in the shedding cavity and the cavitating tip vortex (far field phenomena). Also the lift and drag of the hydrofoil are sufficiently well predicted within $5 \%$ as from the current study (see NACA0015 profile, Twist 11 hydrofoil for Elliptic 11 rake hydrofoil wetted flow results).

On the other hand, more advanced turbulence models like RSM (see [51]) have a high demand on computer power and grid resolution while small time steps are already required for the cavitating flow. Thus for the present investigation that especially addresses numerical simulations for industry requirements the RSM model is not yet suitable.

Concluding, the proposed method is considered to have sufficient capability and is practical/fast/easy to apply to be used in the next step of numerical simulations for the cavitating flow around propellers as required by the marine industry. 


\section{Chapter 6}

\section{Application Challenge}

\subsection{Introduction}

The objective of this Chapter is the prediction of inception and development tip vortex cavitation on marine propellers. An existing numerical method, based on the RANS equations, is used on two representative marine propellers, known to feature tip vortex cavitation.

This chapter contains results that deal with propellers in a uniform flow. The study addresses non-cavitating and cavitating conditions, at model scale as well as on full scale. The numerical simulations in preceding chapters have been performed for relatively "simple" fixed non-rotating geometries. But when dealing with propellers, one has to consider the propeller rotation. Depending on the conditions, a number of approaches are available.

For the numerical simulation of wetted steady flow conditions, use is made of the Moving Frame of Reference (MFR) approach. In the rotating reference frame method, the entire mesh or parts of the mesh rotates at a constant angular velocity about a prescribed axis. This results in Coriolis and centrifugal forces in the momentum equation.

For cavitating flow, a Moving Mesh approach is required due to the unsteadiness of this type of flow. The mesh is rotating by specifying a time-varying position for all or parts of the cell vertices. Now an additional equation called 'geometric conservation law' is solved for the moving coordinates. Further details of the numerics and implementation of the rotary motion in the wetted and cavitating flow condition can be found in [17].

In the current chapter two propeller geometries are analysed : one conventional propeller (4119 DTRC propeller, see [39]) at model scale and one more modern propeller with skew at model as well as full scale (Leading Edge skew propeller, see [66]). 
The first case, the 4119 DTRC propeller, is a benchmark for many CFD predictions of the performance of propellers, see for example the ITTC reports: [1] and [3]. This model propeller is used here to validate predictions of open water performance, pressure and velocity downstream of the propeller inside the tip vortex core, both for wetted and cavitating flow conditions. Also, the test case determines the capability of the computational approach to predict the inception of tip vortex cavitation as well as the development of the tip vortex in the near and far field.

The skew propeller is representative for a propeller of modern design. Such a propeller features a complicated combination of leading edge sheet cavitation, tip vortex and (wake) vortex. Model scale wetted and cavitating flow conditions are studied as well as full scale condition. The latter facilitates the assessment of the Reynolds number scaling effects. This test case will quantify the capability of the computational method to predict: leading edge-tip vortex cavitation and its downstream development, for propellers in uniform inflow at model and full scale.

Since both test cases assume an uniform inflow the time dependency is due to the propeller rotating in the hydrostatic pressure field. The radiated pressure fluctuations due to tip vortex cavitation should be considered in case of a non uniform inlet velocity field. In the following all the numerical simulations of cavitating flows have been carried out for one representative time step. Thus only the effect of blade position can be assessed as far as the unsteadiness of tip vortex is concerned. 


\subsection{DTRC Propeller}

\subsubsection{Geometry}

The first test case of a model scale propeller is one of the propeller geometries used most in the validation of numerical simulations of propeller flows: the 4119 DTRC propeller. For this propeller detailed results are available of LDV measurements conducted by S. Jessup in 1989, see [39]. Note that this experimental investigation is only carried out for wetted flow conditions.

The model scale 4119 DTRC propeller has a diameter of $0.305 \mathrm{~m}, 3$ blades, rotates at $600 \mathrm{RPM}$ and has a hub-diameter ratio of 0.2 . The design condition of the propeller corresponds to advance ratio, $J=0.833$ and a thrust coefficient $K_{t}=0.150$; for which the blades operate at shock free leadingedge flow for the uniform inflow conditions considered. The main geometric data are listed below and in table 6.1 and 6.2 as found in [39].

Diameter, D: $1.00 \mathrm{ft} .(0.305 \mathrm{~m})$

Number of Blades: 3

Hub-Diameter Ration: 0.20

Skew, Rake : none

Design Advance Ratio, J: 0.833

Blade Section Thickness: NACA66 (DTRC Modified)

Blade Section Meanline: NACA, chamber line of $\mathrm{a}=0.8$

Design Thrust Coefficient, $K_{t}: 0.150$

\begin{tabular}{|c|c|c|c|c|c|c|}
\hline $\mathrm{r} / \mathrm{R}$ & $\mathrm{c} / \mathrm{D}$ & $\mathrm{P} / \mathrm{D}$ & Skew & Rake & $t_{M} / \mathrm{C}$ & $f_{M} / \mathrm{C}$ \\
\hline 0.2 & 0.320 & 1.105 & 0 & 0 & 0.2055 & 0.01429 \\
\hline 0.3 & 0.3625 & 1.102 & 0 & 0 & 0.1553 & 0.02318 \\
\hline 0.4 & 0.4048 & 1.098 & 0 & 0 & 0.1180 & 0.02303 \\
\hline 0.5 & 0.4392 & 1.093 & 0 & 0 & 0.09016 & 0.02182 \\
\hline 0.6 & 0.4610 & 1.088 & 0 & 0 & 0.06960 & 0.02072 \\
\hline 0.7 & 0.4622 & 1.084 & 0 & 0 & 0.05418 & 0.02003 \\
\hline 0.8 & 0.4347 & 1.081 & 0 & 0 & 0.04206 & 0.01967 \\
\hline 0.9 & 0.3613 & 1.079 & 0 & 0 & 0.03321 & 0.01817 \\
\hline 0.95 & 0.2775 & 1.077 & 0 & 0 & 0.03228 & 0.01631 \\
\hline 1.0 & 0.0 & 1.075 & 0 & 0 & 0.03160 & 0.01175 \\
\hline
\end{tabular}

Table 6.1: Geometry of Propeller 4119 DTRC

In table $6.1, r / R$ is non-dimensional radius distribution, $c / D$ is the nondimensional chord distribution, $P / D$ is the non-dimensional pitch distri- 


\begin{tabular}{|c|c|c|}
\hline$X_{C},(\% \mathrm{C})$ & $\mathrm{t} / \mathrm{C}$ & $\mathrm{f} / \mathrm{C}$ \\
\hline 0.0000 & 0.0000 & 0.0000 \\
\hline 0.0125 & 0.2088 & 0.0907 \\
\hline 0.0250 & 0.2932 & 0.1586 \\
\hline 0.0500 & 0.4132 & 0.2712 \\
\hline 0.0750 & 0.5050 & 0.3657 \\
\hline 0.1000 & 0.5814 & 0.4482 \\
\hline 0.1500 & 0.7042 & 0.5869 \\
\hline 0.2000 & 0.8000 & 0.6993 \\
\hline 0.3000 & 0.9274 & 0.8635 \\
\hline 0.4000 & 0.9904 & 0.9615 \\
\hline 0.4500 & 1.0000 & 0.9881 \\
\hline 0.5000 & 0.9924 & 1.0000 \\
\hline 0.6000 & 0.9306 & 0.9786 \\
\hline 0.7000 & 0.8070 & 0.8892 \\
\hline 0.8000 & 0.6220 & 0.7027 \\
\hline 0.9000 & 0.3754 & 0.3586 \\
\hline 0.9500 & 0.2286 & 0.1713 \\
\hline 1.0000 & 0.0666 & 0.0000 \\
\hline
\end{tabular}

Table 6.2: Thickness and Camber distribution for Propeller 4119 DTRC

bution, $t_{M} / C$ is the non-dimensional maximum thickness distribution and $f_{M} / C$ is the non-dimensional maximum camber distribution. In table 6.2, $t / C$ is the non-dimensional thickness distribution and $f / C$ is the non-dimensional chamber distribution, see Jessup [39] and Carlton [15] for more details of thr definition of propeller geometry and its characteristics.

The geometry of the blade of the 4119 DTRC propeller is shown in figure 6.1 .

\subsubsection{Computational domain and mesh}

The standard computational domain for a propeller is a domain containing a single blade. Such a domain (see figure 6.3) can be used due to the geometric periodicity of the propeller, provided uniform inlet conditions are considered. The current mesh for the propeller is a multi-block hexagonal mesh, featuring an O-grid type mesh on surfaces around the blade. With such a mesh one can maintain control over the quality of the mesh near the blade and quan- 


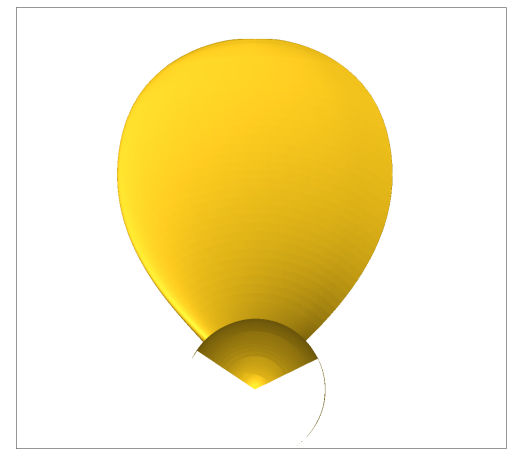

Figure 6.1: Geometry of the blade of propeller 4119 DTRC

tify the condition on the value of the $y^{+}$of the first grid line off the surface. The development of the boundary layer along the blade surface is taken into account by wall functions. The requirement for the $y^{+}$value on the blade surface can be met with an acceptable number of cells in the direction normal to the blade surface. Moreover, the cell aspect ratio and the variation in cell size can be kept within acceptable bounds. The surface mesh of the 4119 DTRC propeller is shown in figure 6.2. Note, that the average surface grid cell is around $1 \mathrm{~mm}$ by $1 \mathrm{~mm}$ in size, i.e. close to the recommended value. Near the leading edge, trailing edge and propeller tip the size of the surface cells is much smaller. The total number of cells obtained for one blade is about 700000 .

The boundary conditions are as follows:

(i) uniform inlet velocity on a plane normal to the axis of the propellers located at two propeller diameters upstream;

(ii) a pressure boundary condition on a plane normal to the propeller axis, located at three propeller diameters downstream

(iii) pressure boundary condition on a circle-cylindrical surface with a radius of two propeller diameters.

\subsubsection{Numerical parameters}

The RANS equations are solved using the SIMPLE algorithm and the MARS differencing scheme. As concluded from the preceding analysis in Chapter 5, the RNG $k-\varepsilon$ turbulence model is used since it is the only turbulence model investigated that is capable to predict accurately the drag and the cavitation 
phenomenon.

The rotation of the propeller is accounted for using the Multiple Rotating Reference Frames approach as described in [17], which is appropriate for wetted flow.

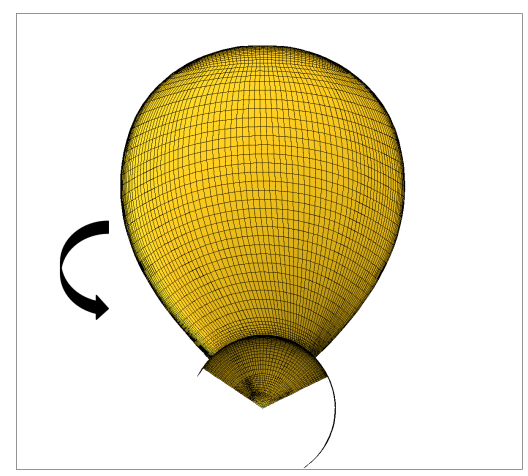

Figure 6.2: Propeller 4119 DTRC. Blade surface mesh: 8000 cells on the blade surface

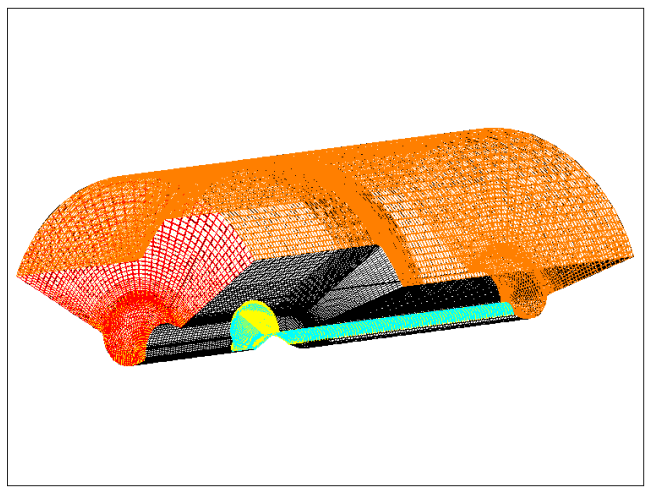

Figure 6.3: Computational domain for one blade of Propeller 4119 DTRC 


\subsubsection{Results wetted flow}

The results of the numerical simulations for wetted flow, for various advance ratios, are analysed by means of performance curves. A detailed investigation is carried out at the design condition specifically focussed on assessing the effects of the the tip vortex.

The propeller Reynolds number as described by Kuiper in [43] is defined as:

$$
R e_{n}=\frac{n D^{2}}{\nu}
$$

The advance ratio $J$ is defined as:

$$
J=\frac{V}{n D}
$$

with $V$ the advance/inlet velocity, $n=R P M / 60$ is the number of propeller rotations per second and $D$ is the propeller diameter.

For the present test case $R e_{n}=930250$. In equation $6.1 n$ equals 10 , which is the number of rotations per second, $D$ is the diameter, equal to $D=0.305 \mathrm{~m}$, and $\nu=1.0 * 10^{-6} \mathrm{~m}^{2} / \mathrm{s}$ is the kinematic viscosity of water.

When the inlet velocity equals $V=2.54 \mathrm{~m} / \mathrm{s}$, the advance ration is $J=0.833$. The distribution of $y^{+}$is shown in figure 6.4 for both the suction and pressure side. It shows that the value of $y^{+}$is well within the recommended value between 30 and 100, as given in [17].

In figure 6.5 the corresponding residuals of the equations of conservation of mass, momentum and turbulence kinetic energy are presented.

Propeller performance is described by a set of non-dimensional coefficients, namely the thrust coefficient and the torque coefficient, see for example Carlton $[15]$.

The thrust and torque coefficients are defined as:

$$
\begin{aligned}
K_{t} & =\frac{\text { Thrust }}{\rho n^{2} D^{4}} \\
K_{q} & =\frac{\text { Torque }}{\rho n^{2} D^{5}}
\end{aligned}
$$

These dimensionless quantities are, for a given geometry, a function of advance ratio $J$ and Reynolds number $\operatorname{Re}_{n}$.

The results of the numerical simulations of the flow over the 4119 DTRC propeller at different inlet velocities have been analysed in terms of the thrust 


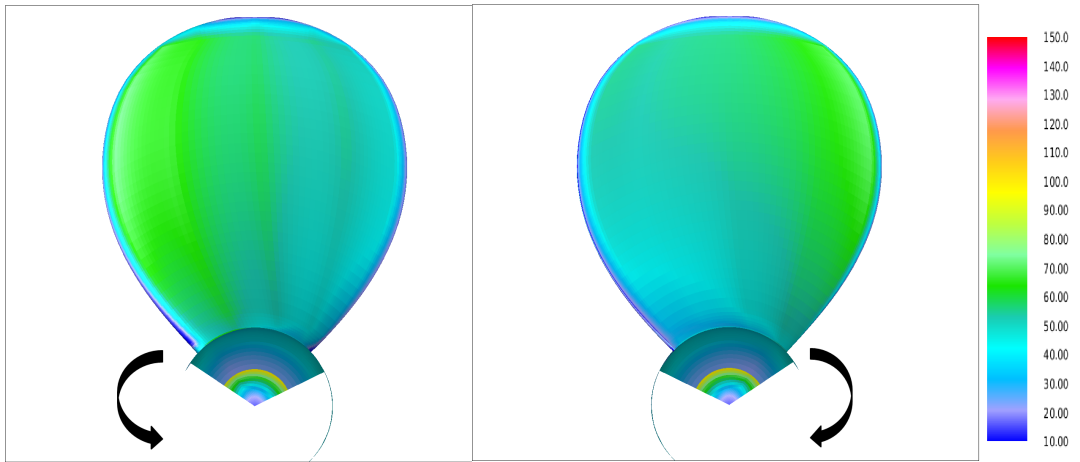

Figure 6.4: 4119 DTRC propeller. Distribution $y^{+}$on suction (left) and pressure (right) sides, $J=0.833, \operatorname{Re}_{n}=930250$

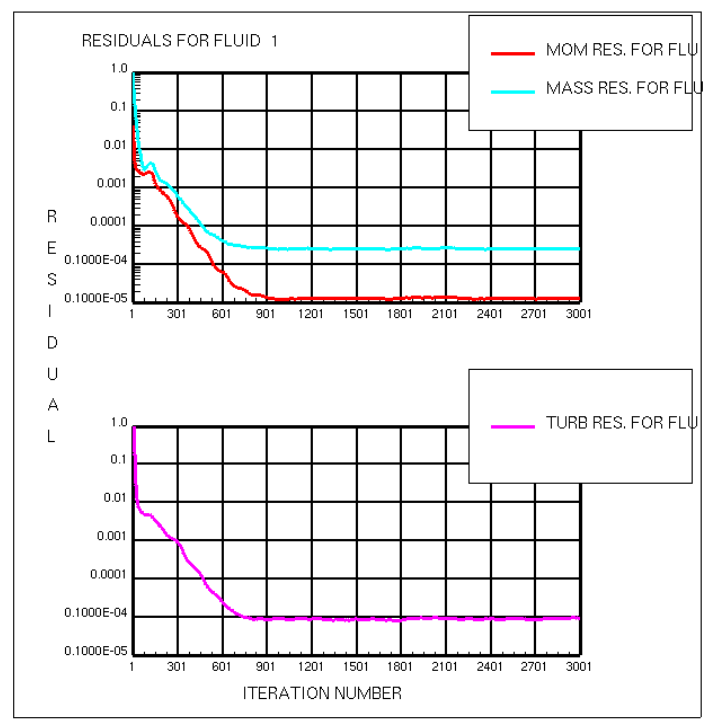

Figure 6.5: 4119 DTRC propeller. Convergence of residuals of equations for conservation of mass, momentum and turbulence kinetic energy, $J=0.833$, $\operatorname{Re}_{n}=930250$

and torque coefficients. These results are presented as part of the open water curves shown in figure 6.6. From this figure it is clear that the numerical method is able to reproduce the results of the model tests in terms of the 
global coefficients characterising the propeller performance.

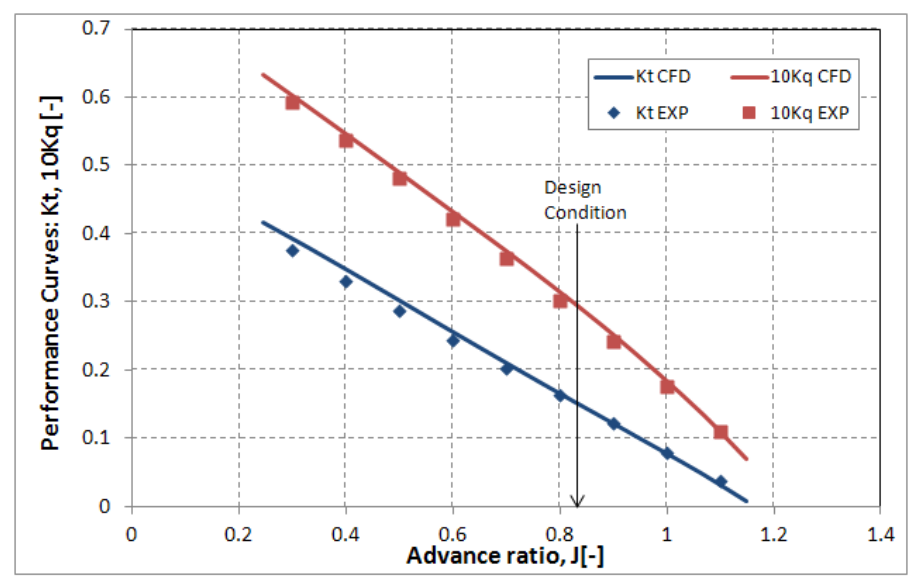

Figure 6.6: Performance curves for 4119 DTRC propeller. Thrust coefficient $K_{t}$ and torque coefficient $K_{q}, \mathrm{Re}_{n}=930250$. Comparison of results of numerical simulations and measurements

At design condition $(J=0.833$, RPM $=840)$ the numerical simulations predict for the 4119 DTRC propeller a thrust coefficient of $k_{t}=0.150$, the same value as the propeller design value listed in table 6.3.

\begin{tabular}{|c|c|c|}
\hline Case & $K t$ & $10 K q$ \\
\hline Experiment & 0.154 & 0.290 \\
\hline Simulations & 0.154 & 0.300 \\
\hline
\end{tabular}

Table 6.3: 4119 DTRC propeller. Predicted and measured thrust and torque coefficients at design condition $J=0.833, \mathrm{Re}_{n}=930250$

In table 6.3 , for the design condition $J=0.833$ the predicted thrust and torque coefficients are compared with measured data from [39]. The thrust coefficient agrees quite well. The torque is slightly over-predicted (about $3.3 \%$ ). Note that the experimental value requires some error estimation. This result is typical for predicting propeller torque using CFD. It is related to the over-prediction of the drag, as already discussed for the NACA 0015 profile.

The distribution of the pressure coefficient (defined in equation 6.5) on the 
suction and pressure side of the blade, is presented in figure 6.7. Moreover, for the blade section at $r=0.7 R$, the predicted distribution of the pressure coefficient is compared with the measured values in figure 6.8. From figure 6.7 the presence of the tip vortex can be observed as the low pressure region at the mid chord position in the tip region.

$$
\begin{gathered}
C_{p}=\frac{p-p_{\infty}}{\frac{1}{2} \rho(n D)^{2}} \\
C_{p, r}=\frac{p-p_{\infty}}{\frac{1}{2} \rho\left(V_{R}\right)^{2}} \\
V_{R}^{2}=V^{2}+(2 \pi n R)^{2}
\end{gathered}
$$

Figure 6.8, shows slight differences between the results of experiments and numerics, specifically at the leading edge. This difference can be related to: measurements errors, the difference in the regime of the flow in measurement (laminar flow is usually present over part of the blade in measurements) versus numerical simulation (fully turbulent flow), slight differences in the blade geometry and maybe also small errors in data gathering, see [39]. Still the differences are small and have limited influence on the performance and tip vortex as shown further on.

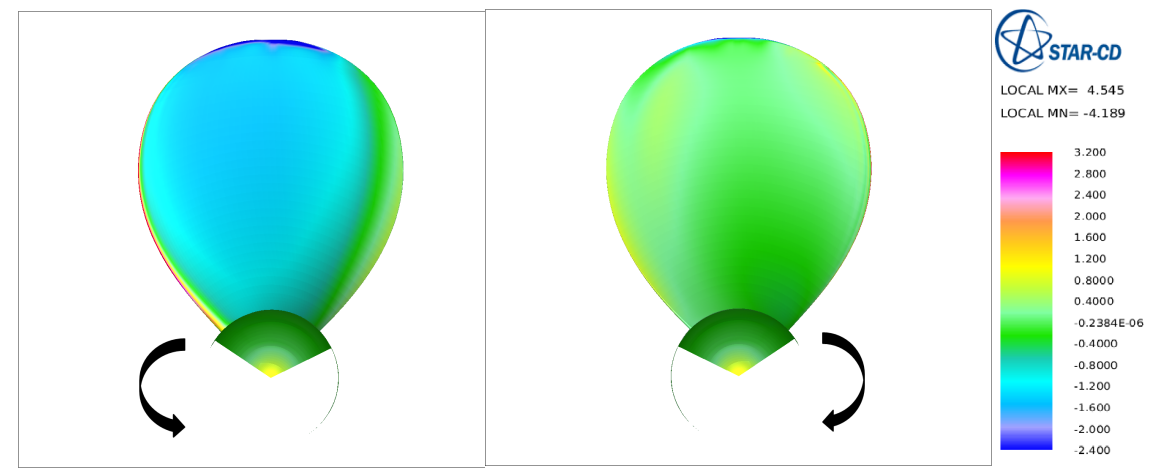

Figure 6.7: 4119 DTRC propeller. $C_{p}$ on suction (left) and pressure (right) sides, $J=0.833, \operatorname{Re}_{n}=930250$ 


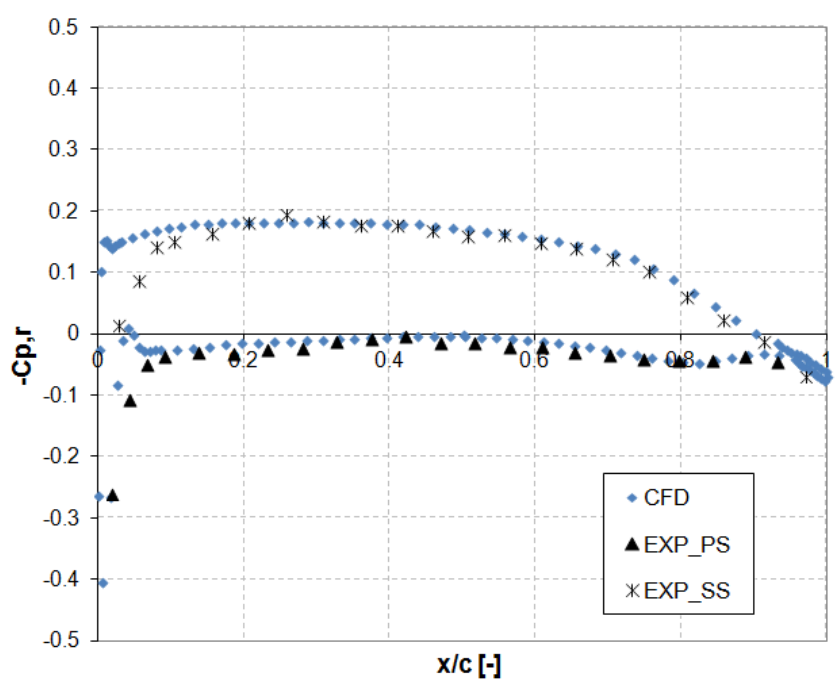

Figure 6.8: 4119 DTRC propeller. Predicted and measured distribution of the pressure coefficient $C_{p, r}$ at $r=0.7 R, J=0.833, \operatorname{Re}_{n}=930250$

For the study of the tip vortex, a new refined mesh has been prepared. The one blade propeller mesh from figure 6.3 is repeated in a periodic fashion 3 times. Subsequently, one of the blades of the propeller is refined three times in the tip region based on the method described in chapter 3. The initial solution is analysed and the cells that satisfy two criteria: 1 . the value of the Q-factor is higher than a certain threshold value and 2. the pressure is lower than a certain threshold value. Then a numerical simulation is performed for the first refined mesh and a second selection of cells based on the Q-factor and minimum pressure (higher and lower, respectively compared to the values for the previous iteration) are selected to be refined. The last refinement is made based on the numerical solution for the second refined grid, selecting again the cells with a higher value of the Q-factor and a lower value for the pressure than for the previous iteration. The third refined mesh is found and the last selection of cells to be refined determined. After this third refinement the number of cells is almost double the number of cells of the initial grid. Another refinement is not feasible, see table 6.4 for the number of cells corresponding to each grid. Note that the refinement iteration runs up to $z / R=0.7$ downstream of the propeller plane, see figure 6.9.

In the following the influence of the grid resolution on the tip vortex is 

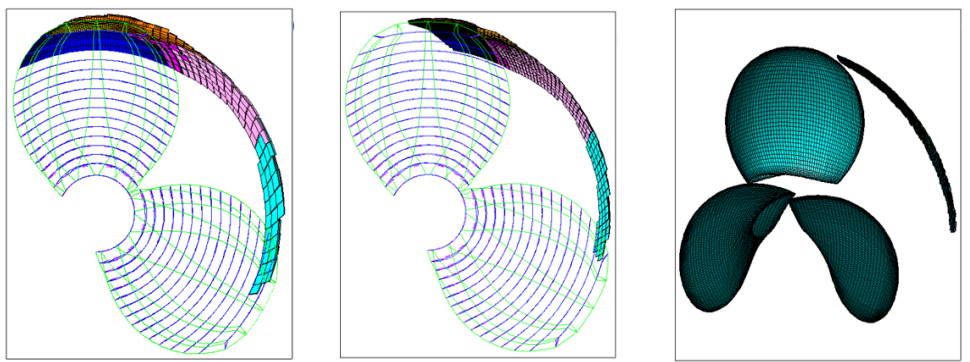

Figure 6.9: 4119 DTRC propeller, $J=0.833, \mathrm{Re}_{n}=930250$. First, second and third iteration for selection of cells to be refined

\begin{tabular}{|c|c|}
\hline Grid & Number of cells \\
\hline Initial & $1.4 * 10^{6}$ \\
\hline G1 & $1.6 * 10^{6}$ \\
\hline G2 & $2.2 * 10^{6}$ \\
\hline G3 (final) & $2.5 * 10^{6}$ \\
\hline
\end{tabular}

Table 6.4: 4119 DTRC propeller. Number of cells for grids with improved resolution of tip vortex

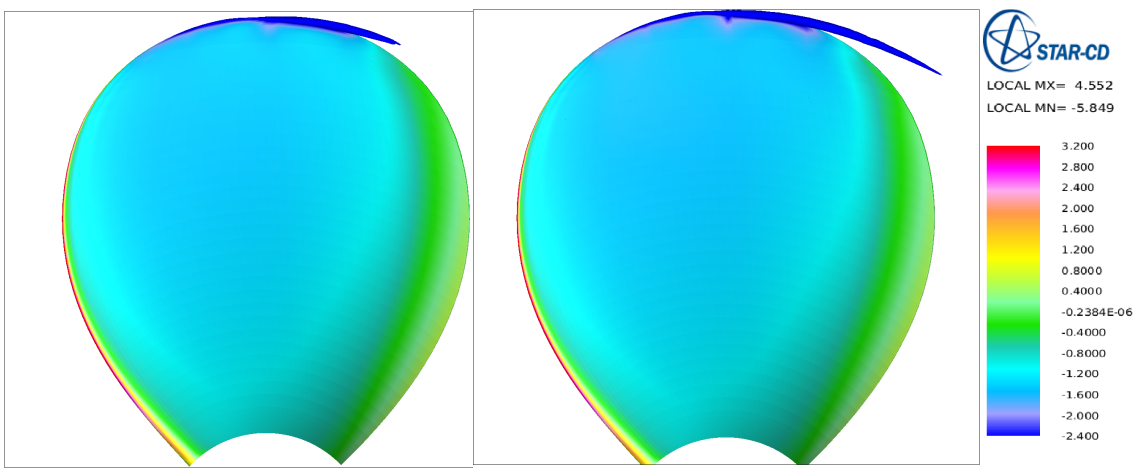

Figure 6.10: 4119 DTRC propeller. $J=0.833, \mathrm{Re}_{n}=930250, K_{t}=0.150, C_{p}$ on pressure side, initial (left) and refined G3 (right) grids

analysed in terms of: pressure coefficient, velocity field and Q-factor. This analysis has been carried out for the design condition $(J=0.833$ and $R e=$ 930250).

In figure 6.10 , the pressure coefficient distribution on the blade are shown 
for the initial grid and for the refined (G3) grid, respectively. The contour of the $C_{p}=-2.4$ is also shown for the initial and refined (G3) grids. As expected, the extent of the region of low pressure at the tip region is larger for the refined mesh (G3).

Figure 6.11 shows the distribution of the Q-factor and the pressure coefficient along the circular arc $r / R=0.958$ through the tip vortex core, in the plane normal to the axis of propeller $z=0.328 R$, downstream of the propeller. The distribution of the azimuthal velocity in the plane $z=0.328 R$ along the circular arc $r / R=0.958$ through the tip vortex core of propeller 4119DTRC is presented in figure 6.12. From this figure the location of the tip vortex is evident. The magnitude of the peak of the azimuthal velocity is lower than the measured values. However, the circulation of the vortex core does not differ much. This is mainly due to grid resolution at this location close to the blade but also due to the turbulence modeling further downstream. In the vortex core the distribution of the Q-factor and of the azimuthal velocity corresponds very well, i.e. the region with high values of the Q-factor $(\Delta \theta=10 \mathrm{deg})$ is the region in the distribution of the azimuthal velocity with a large gradient (solid-body rotation). In the experimental data the vortex core appears to have a smaller diameter $(\Delta \theta=5 \mathrm{deg})$.

Since the grid refinement was made based on low pressure coefficient and high Q-factor, the azimuthal velocity component is not very well captured as visible from figure 6.13 , which shows the velocity and pressure fields and corresponding grid resolution in plane $z=0.328 R$.

The development in downstream direction of the minimum pressure coefficient and the maximum Q-factor in the tip vortex is plotted in figure 6.14. Figure 6.14 shows, as for the Elliptic 11 rake test case, that in the results of the numerical simulation the propeller tip vortex decays fast downstream, especially in terms of the value of the Q-factor. This is partially due to insufficient grid resolution but also due to a strong dissipation of the turbulence in the vortex core introduced by the RANS approach, see [34].

The radial location of the tip vortex determined from numerical results is plotted in figure 6.15. Included in this figure is the experimental observation [39]. The predicted radial location of the vortex core is well in agreement with the measurements at least close to the propeller tip, while further downstream the location deviates due to the fast decay of the vortex in the numerical simulations. At the downstream location of $z=0.328 R$ considered before, the CFD results over-predict the radial position with $1.38 \%$ relative to the measurements. 


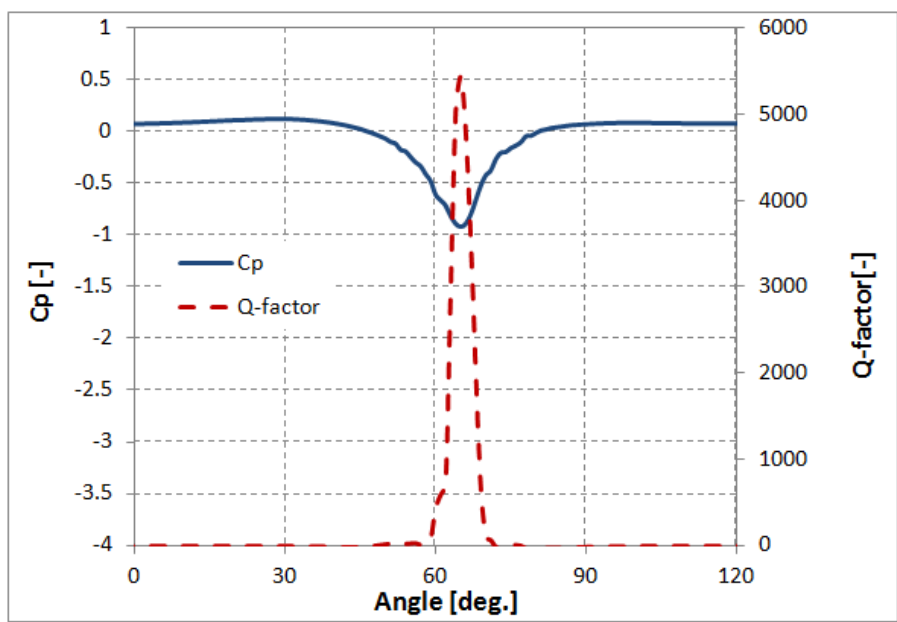

Figure 6.11: 4119 DTRC propeller. Pressure coefficient and Q-factor in plane $z / R=0.328$ downstream along $r / R=0.958$ through the vortex core, $J=0.833, \operatorname{Re}_{n}=930250, K_{t}=0.150$, refined G3 grid

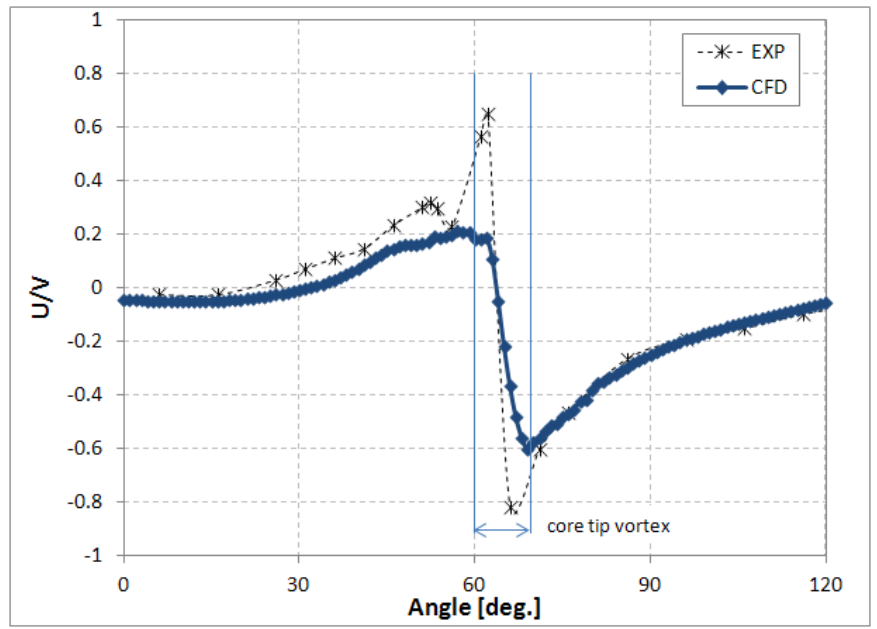

Figure 6.12: 4119 DTRC propeller. Azimuthal velocity distribution along $r / R=0.958$ through the vortex core, in plane $z / R=0.328$ downstream. $J=$ $0.833, \mathrm{Re}_{n}=930250, K_{t}=0.150$. Prediction (G3 grid) versus measurements 


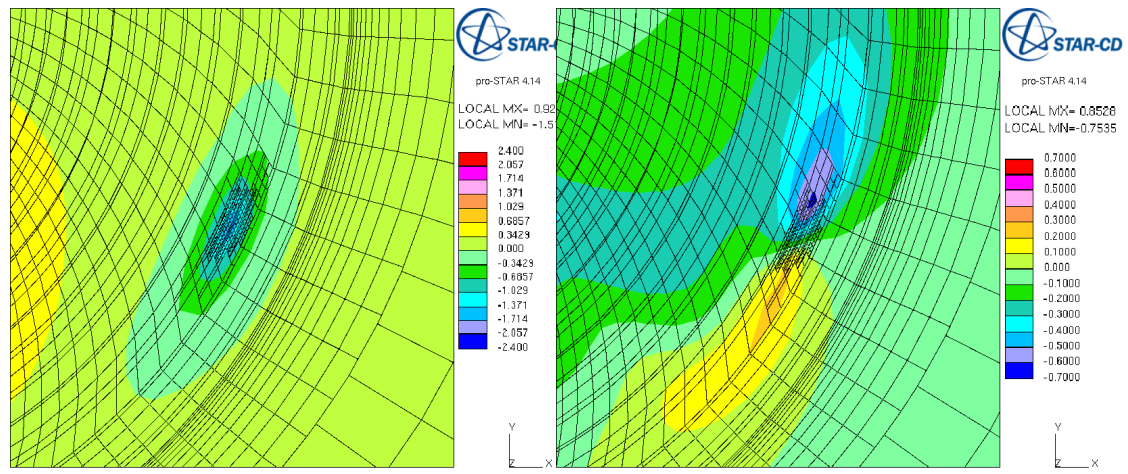

Figure 6.13: 4119 DTRC propeller. $J=0.833, \mathrm{Re}_{n}=930250, K_{t}=0.150$, $\mathrm{Cp}$ (left) and azimuthal velocity $\mathrm{U} / \mathrm{V}$ (right) in plane $\mathrm{z} / \mathrm{R}=0.328$ downstream of propeller, G3 mesh

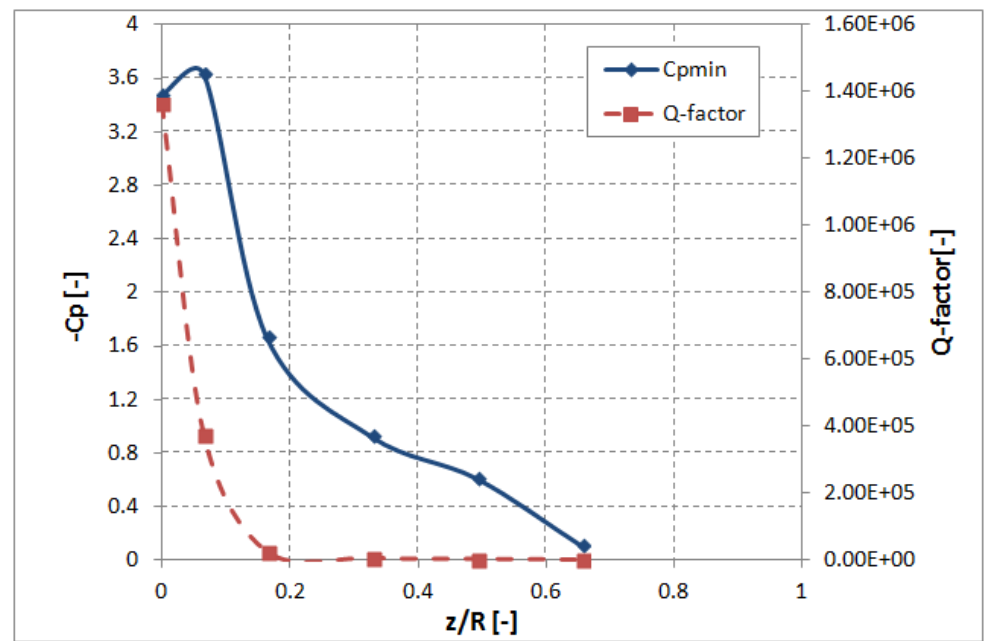

Figure 6.14: 4119 DTRC propeller. Pressure coefficient and Q-factor in core of the vortex, downstream of propeller, at $J=0.833, \mathrm{Re}_{n}=930250$ and $K_{t}=0.150$, G3 mesh

Using the experimental data corresponding to figure 6.15 and the vortex core radius from [39], the tip vortex can be reconstructed. Figure 6.16, presents the tip vortex reconstructed from experimental data [39], the measurement 


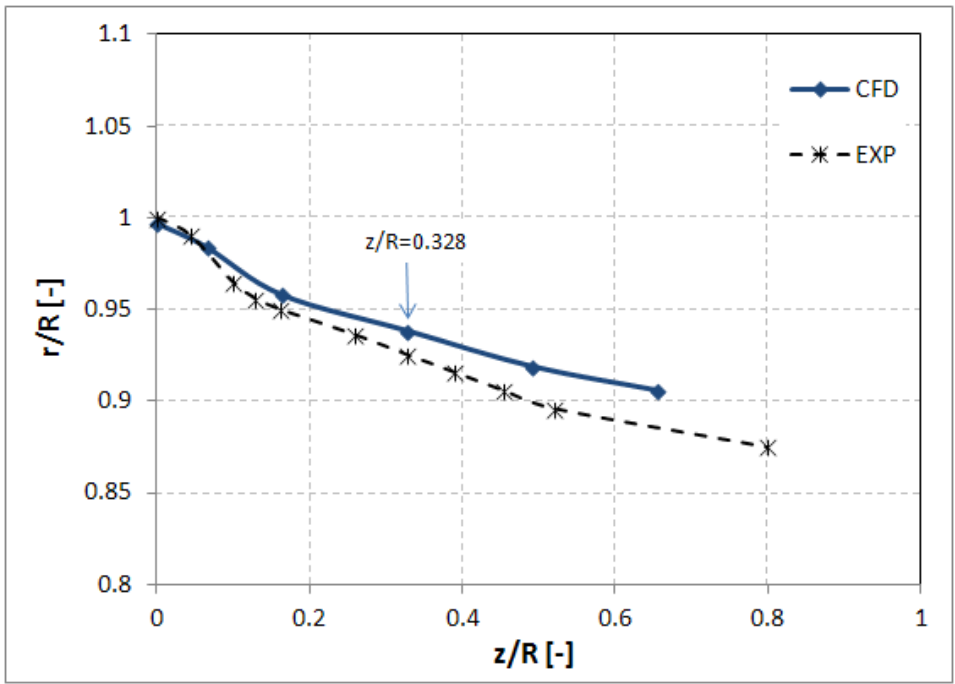

Figure 6.15: 4119 DTRC propeller. Location of the tip vortex core as function of downstream distance. $J=0.833, \mathrm{Re}_{n}=930250$ and $K_{t}=0.150$. Prediction and experiment, G3 mesh

of the radial velocity together with the tip vortex reconstructed from the numerical simulations, i.e. the location of iso-contour $C_{p}=-2.4$. It is seen that the predicted location of the tip vortex, close to the tip of the propeller is in-line with the measured location. From the numerical results it appears to follow that if the extent of tip vortex core is defined in terms of an iso- $C_{p}$ contour, the thickness of the tip vortex is over-estimated and is fast decreasing in downstream direction. The over-prediction of the core size is mainly related to the turbulence diffusion since at this location the grid convergence has been reached, while also the measurement error should be considered. Note also that selection of different iso- $C_{p}$ contour is influencing the results fot the downstream development of the tip vortex location and core size. Note that in the experiment the location of the tip vortex core is determined from the radial velocity measurements.

The case of wetted flow is the main validation case for propeller 4119 DTRC. This condition is used as a starting point for the numerical simulations for cavitating conditions and for analysing the influence of cavitation on the tip vortex. 


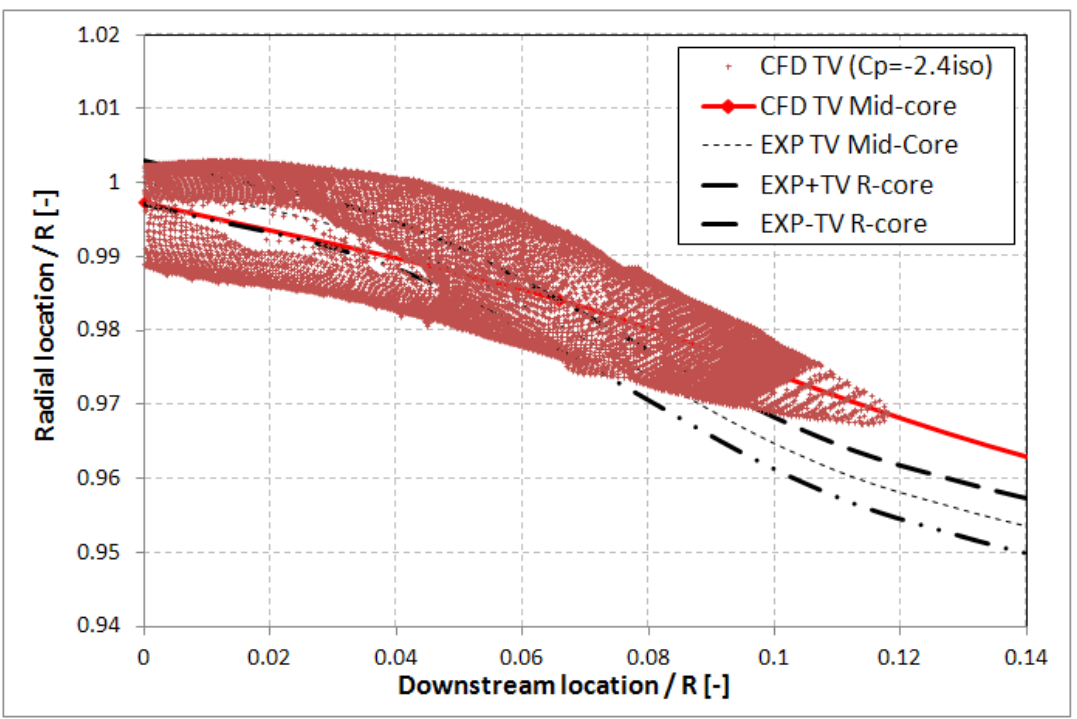

Figure 6.16: 4119 DTRC propeller. Radial location of the tip vortex core at $J=0.833, \operatorname{Re}_{n}=930250$ and $K_{t}=0.150$, G3 gird. Simulation and experiment [39]

\subsubsection{Results for cavitating flow}

\section{Tip vortex cavitation inception}

Two main processes are important when dealing with tip vortex cavitation: the inception of the tip vortex and, once the cavity is initiated, the downstream development of the cavitating tip vortex. Knowledge on the inception of the tip vortex cavitation is required in the design process of propellers. Cavitation needs to be avoided at least for the design condition of the investigated propeller. For detection of the inception of tip vortex cavitation, the visual development of the tip vortex is important (close to the blade tip). Visual inspection is important far downstream to capture the vortex interaction with the ship hull or the rudder. The main phenomena associated with tip vortex cavitation are noise and erosion. The variation of the shape of the cavitating tip vortex in non-uniform inflow results in the radiated pressure field which causes noise and vibrations.

For predicting inception of tip vortex cavitation using numerical simulations, results for wetted flow in terms of a minimum in pressure should give suffi- 
cient information, as outlined in the following.

The minimum pressure coefficient is considered for the 4119 DTRC propeller at design condition $(J=0.833)$ and two extra conditions $(J=0.656$ and $J=0.738)$. Figure 6.17 presents the minimum pressure coefficient $\left(-C p_{\min }=\sigma\right)$ as function of the advance ratio $(J)$ for the initial grid (blue dots) and refined G3 grid (red dots) in comparison with the experimental results. From the cavitation inception chart (figure 6.17), it is found that at design condition $(J=0.833)$ the prediction by CFD of the inception of the cavitation of the tip vortex, for the initial grid, takes place around 1.0R at a cavitation number around $\sigma=4.2$. The cavitation inception of the detached tip vortex at $\sigma \approx 5$ is only captured by CFD on the refined grid. Figure 6.17 shows the same trend for the initial grid as seen in the values for grid G3, i.e. the grid refined in the region of the tip vortex.

It appears that employing the grid of the 4119 DTRC propeller refined in the tip vortex region, it is possible to predict the cavitation inception of the detached tip vortex. In contrast, for the coarser mesh (initial grid) CFD predicts inception at the propeller surface and not in vortex core.

It should be noted that the measurements are also influenced by the water quality (number of nuclei) as presented in figure 6.18, see ITTC 21st Cavitation Committee [2]. From this chart it is clear that cavitation inception is more influenced by decreasing $J$ than by increasing $\sigma$. Thus, the uncertainty of the measurement is higher at low $\mathrm{J}$ and high cavitation number. The agreement of the measurements with predicted values (figure 6.17) for the cavitation inception of the tip vortex is within practical tolerances for all investigated advance ratios and grids when taking into account the effect of water quality (figure 6.18). 


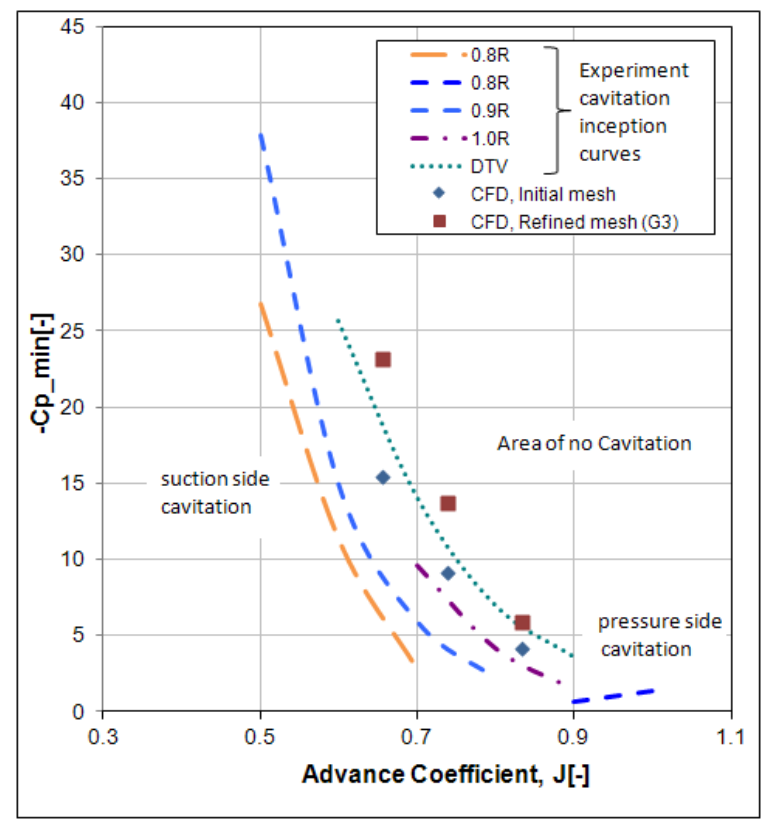

Figure 6.17: 4119 DTRC propeller. Cavitation inception curves obtained from experiments (DTV: detached tip vortex) and from numerical simulations for wetted flow, minimum pressure coefficient at $J=0.833, J=0.738$ and $J=0.656$

\section{Development in downstream direction of tip vortex cavitation}

Once the cavitation is initiated, i.e. in the vortex core the minimum pressure coefficient equals the cavitation number, the cavitation develops within the tip vortex core that forms at the tip of the propeller blade. The present section analyses the extent of the tip vortex cavitation, its shape and its influence on the propeller performance of propeller 4119 DTRC.

Numerical simulations of cavitating flow about the rotating propeller involves moving mesh computations in order to account for the rotation of the propeller. This approach involves the selection of time steps in terms of increment in degrees azimuth angle. For the current simulations the maximum step (in blade position) used is 0.25 degrees. Then the time step in seconds is defined by:

$$
\Delta t=0.25 * \frac{1}{n} * \frac{1}{360}
$$




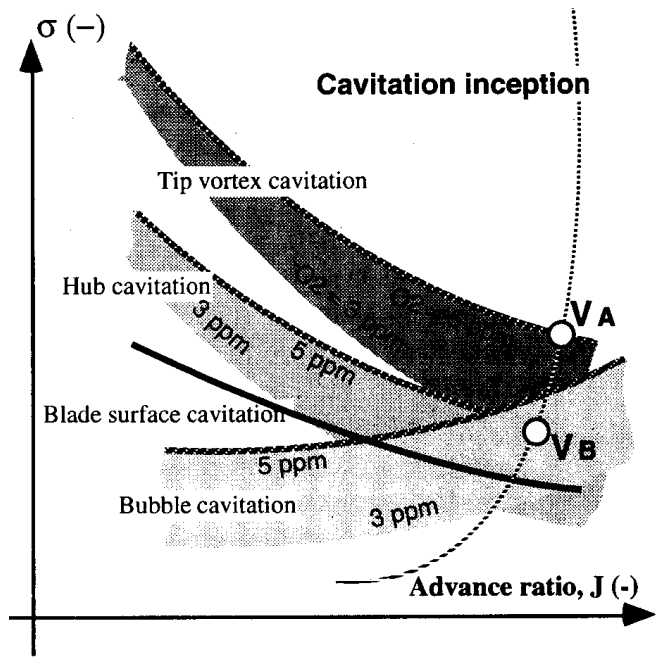

Figure 6.18: Influence of water quality on the cavitation inception bucket, from 21st ITTC 1996 found in [2]

Since the data from experimental investigations of the cavitating flow over the 4119 DTRC propeller are not available to the author, only a numerical study in which numerical results for cavitating flow are compared with numerical results for the case of wetted flow. Two numerical investigations have been performed for cavitating flow: (1) determining the influence of the cavitating tip vortex on the propeller forces, pressures and velocities and (2) determining the stream-wise development of the cavitating tip vortex and comparing it with results of the wetted flow for the development of the tip vortex.

Since validation material is not available, the selected cavitation number can be chosen truly. The condition is that the chosen cavitation number is below the cavitation number at which the tip vortex starts to cavitate. From figure 6.17 it follows that $\sigma_{i}=5$ so that the tip vortex will cavitate at this and lower values of $\sigma$. The simulations have been performed for $\sigma=2.4$. The cavitation number is defined as:

$$
\sigma=\frac{p_{\infty}-p_{v}}{\frac{1}{2} \rho V_{\infty}^{2}}
$$

In equation $6.9, p_{\infty}$ is the outlet pressure which is chosen equal to $10000 \mathrm{~Pa}$. 
The density is $\rho=1000 \mathrm{~kg} / \mathrm{m}^{3}$, the vapor pressure at $T=297 \mathrm{~K}$ is equal to $p_{v}=2370 \mathrm{~Pa}$ and the inlet velocity is $V=2.54 \mathrm{~m} / \mathrm{s}$.

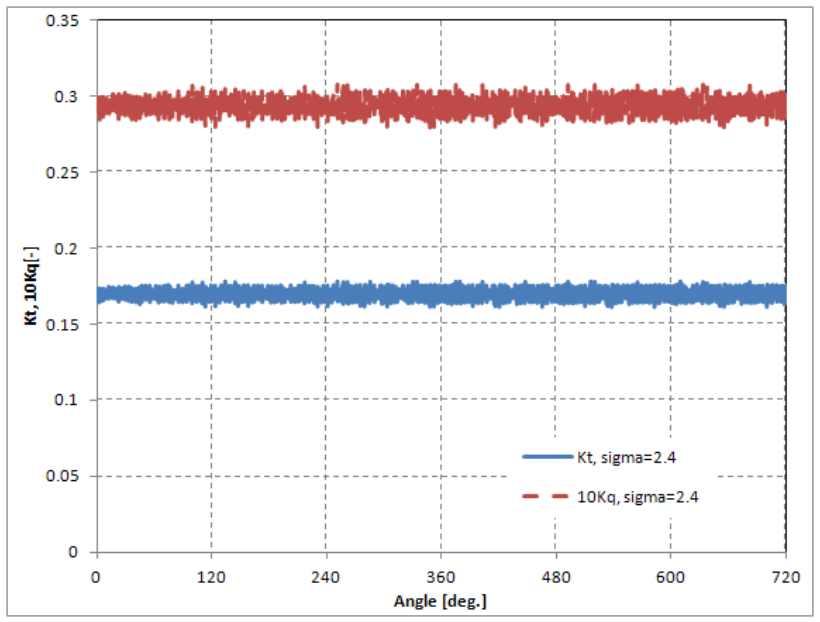

Figure 6.19: Propeller 4119DTRC, $V=2.54 \mathrm{~m} / \mathrm{s}, \quad \mathrm{J}=0.833, \quad \mathrm{RPM}=840$, $\mathrm{Re}_{n}=930250$. Variation of thrust and torque coefficients, cavitating flow $\sigma=2.4$, grid G3

Numerical simulations for cavitating propellers are monitored by means of the history of the force coefficient. Figure 6.19 , shows the variation in time of the thrust and torque coefficients during two propeller revolutions. Note that, the oscillations (around a mean value) in the force are numerical oscillations, typical for moving mesh simulations within Star-CD.

It follows from figure 6.19 that the 4119 DTRC propeller mean thrust and torque coefficients (at the design condition $\mathrm{J}=0.833$ ) are $k_{t}=0.17$ and $k_{q}=0.0295$, respectively. These values are compared with the ones for wetted flow in table 6.5. It is found that due to cavitation the torque has slightly decreased with about $1.5 \%$, while the thrust has increased $10 \%$. The increase in thrust is an expected result for cases of steady flow with small regions with cavitation because in cavitating flows the extent of the low pressure region is slightly larger than for wetted flows. This effect of cavitating flow gives a higher thrust while the torque is not affected very much.

The pressure distribution on the suction side of the blade and the corresponding pressure coefficient iso-contour $C_{p}=-2.4$ of the 4119 DTRC propeller 


\begin{tabular}{|c|c|c|}
\hline Flow & Kt & $10 \mathrm{Kq}$ \\
\hline Wetted & 0.154 & 0.300 \\
\hline Cavitating & 0.170 & 0.295 \\
\hline
\end{tabular}

Table 6.5: Propeller 4119DTRC. $J=0.833, \mathrm{Re}_{n}=930250$, Thrust and torque coefficients, wetted flow and cavitating flow, G3 mesh

in cavitating flow, is presented in the left side of figure 6.20. In comparison to the surface pressure distribution for the wetted flow shown in figure 6.10, one observes the influence of the cavitation on the pressure coefficient, in the extent of the region of minimum pressure coefficient (iso-surface $=-2.4$ ) in the tip region. Also, in figure 6.20 the corresponding vapor content (iso-surface $\alpha=0.01)$ is plotted. This shows a similar shape as the iso contour for pressure coefficient $C_{p}=-2.4$ while the cavitating tip vortex appears to be thicker and slightly shorter.
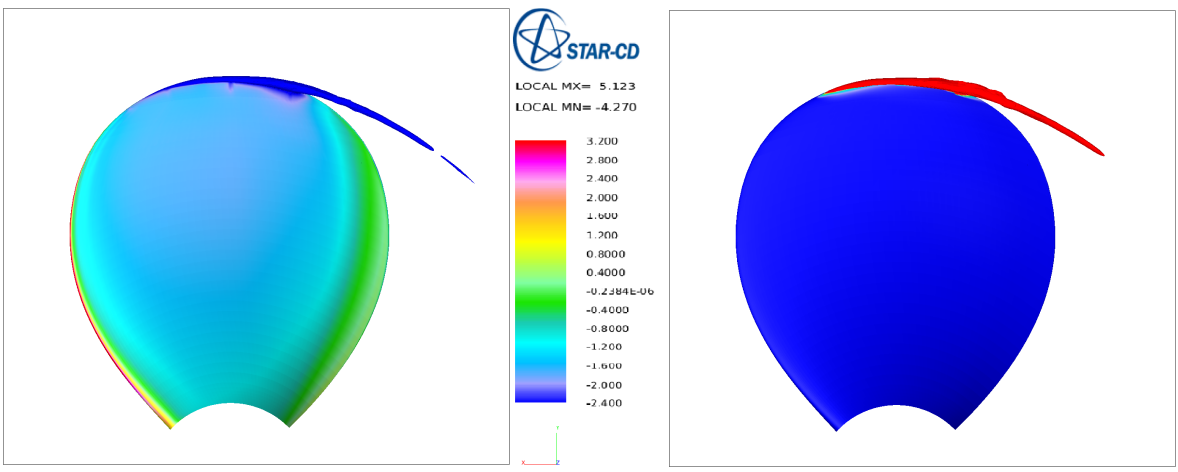

Figure 6.20: Propeller 4119 DTRC. $J=0.833, \operatorname{Re}_{n}=930250, C_{p}$ (left) and vapor fraction iso-surface $\alpha=0.01$ (right), cavitating flow $\sigma=2.4$, G3 mesh

In the case of cavitating flow, the development of the minimum pressure coefficient in downstream direction compared to the case of wetted flow is presented in figure 6.21. This figure shows mainly a lower minimum pressure coefficient for the case of cavitating flow, except in the tip region where cavitation inception is initiated (cavitation inception is reached and the pressure is not decreasing below inception cavitation number).

Figure 6.22 shows the flow in the tip vortex in terms of the pressure coefficient and the vapor content. The corresponding distribution of the azimuthal velocity through the vortex core is presented in figure 6.23 . This distribution 
for cavitating flow is compared with the results for wetted flow at the same representative downstream location $(z=0.328 R)$. As observed for the Elliptic 11 rake hydrofoil, the occurrence of cavitation increases the magnitude of the pressure coefficient and the velocity in the vortex core. However, it appears that the orientation of the vortex is not affected very much be cavitation.

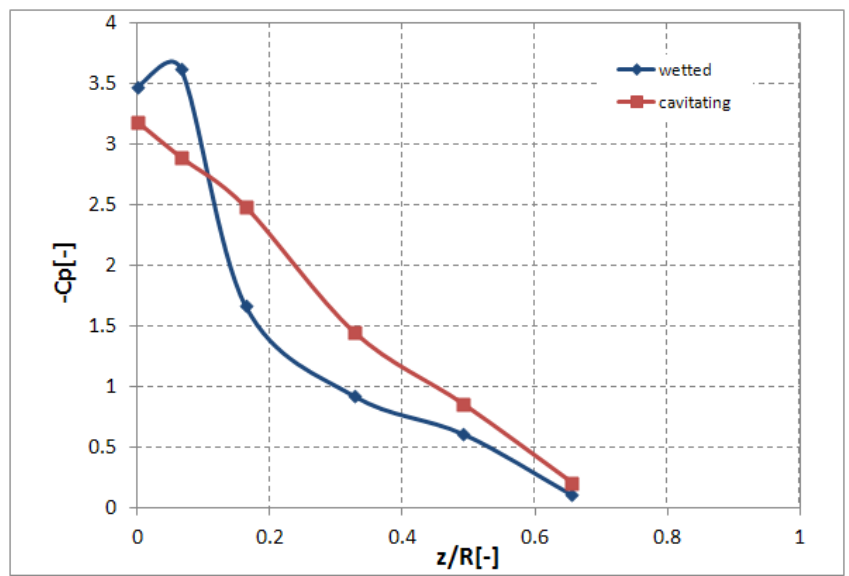

Figure 6.21: Propeller 4119DTRC. $J=0.833, \operatorname{Re}_{n}=930250$, minimum pressure coefficient as function of $\mathrm{z} / \mathrm{R}$ downstream of propeller. Wetted flow and cavitating flow at $\sigma=2.4$, G3 mesh

The radial location of the cavitating tip vortex, corresponding to the pressure iso-surface $C_{p}=-2.4$, is plotted in figure 6.24 both for the wetted flow and for the cavitating flow. From this figure it is indeed quite clear that the cavitating tip vortex core is larger than the tip vortex core in case of wetted flow, both in terms of thickness and length.

However, note that the result of figure 6.24 is strongly dependent on the mesh used, and that in reality the vortex core extends much further downstream, actually over several revolutions of the spiral tip vortex.

Note that, the current test case is not appropriate for studying the unsteady features of the tip vortex since the inlet velocity field is uniform resulting in a tip vortex that is stationary. The only unsteadiness in the tip vortex is due to the hydrostatic pressure. Thus in the following, the tip vortex is studied 


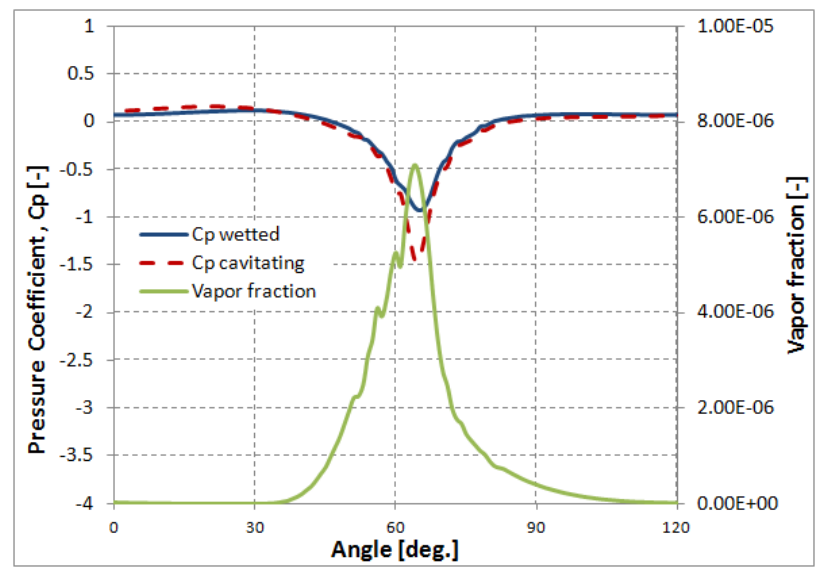

Figure 6.22: Propeller 4119DTRC. $J=0.833, \mathrm{Re}_{n}=930250$, distribution of pressure coefficient and vapor fraction along arc $r=0.95 R$ through the vortex core in plane $z=0.328 R$. Wetted and cavitating flow at $\sigma=2.4$, G3 mesh

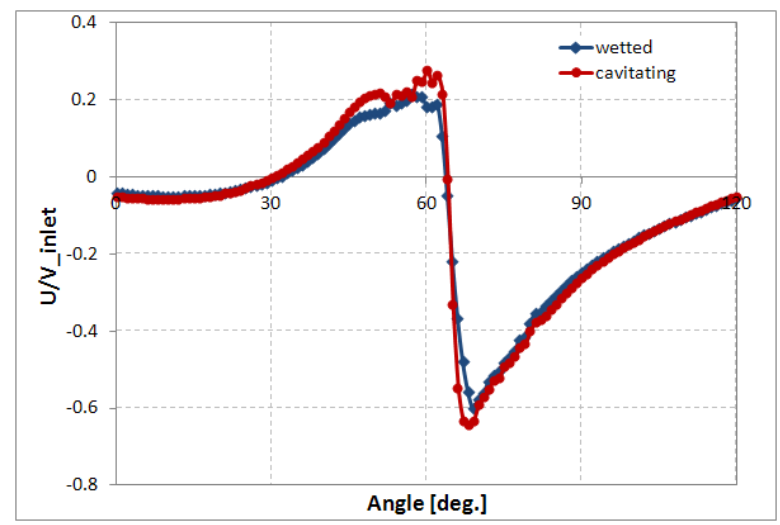

Figure 6.23: Propeller 4119 DTRC. $J=0.833, \operatorname{Re}_{n}=930250$, distribution of azimuthal velocity along arc through vortex core in cross-flow plan $z=$ $0.328 R$. Wetted and cavitating flow at $\sigma=2.4$, G3 mesh

in blade positions corresponding to the 9,6 and 3 o'clock position in comparison to the 12 o'clock position (analysed in the previous charts). In figures 6.25 and 6.26 , the pressure coefficient iso-surface $C_{p}=-2.4$ corresponding 
to the 12, 9, 6 and 3 o'clock blade position is presented. These pictures show a stationary tip vortex that slightly decreases in thickness and length from the 12 o'clock to the 6 o'clock position (see also figure 6.27 in which the tip vortex core is represented in terms of the radial location downstream) and increases again at the 3 o'clock position.

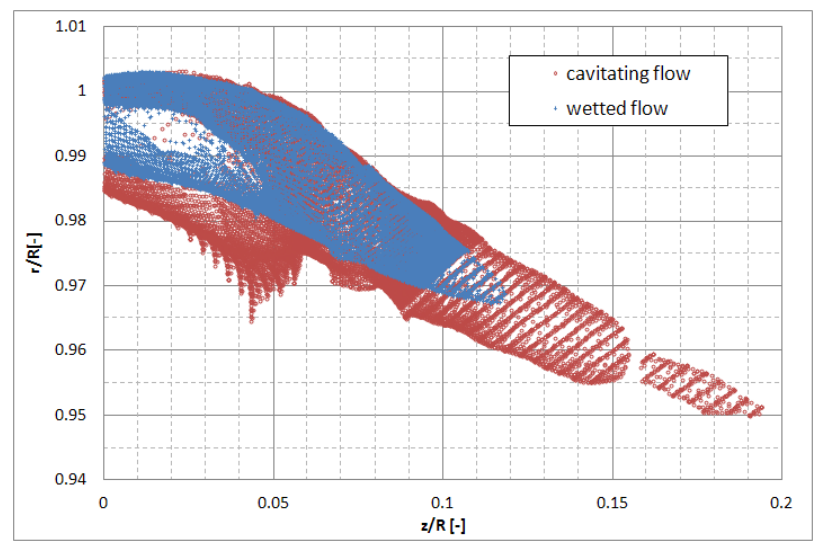

Figure 6.24: Propeller 4119DTRC, $\operatorname{Re}_{n}=930250$. Predicted radial location of the tip vortex core (based on $C_{p}=-2.4$ iso-surface) downstream. Wetted and cavitating flow, G3 mesh
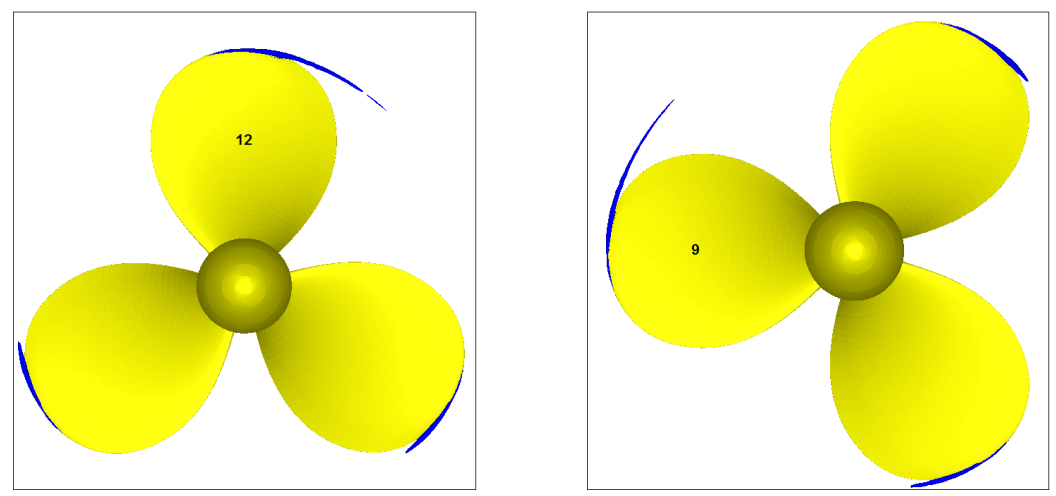

Figure 6.25: Propeller 4119 DTRC. $J=0.833, \operatorname{Re}_{n}=930250, C_{p}=-2.4$ at 12 o'clock (left) and 9 o'clock (right) positions, cavitating flow $\sigma=2.4$, G3 mesh 

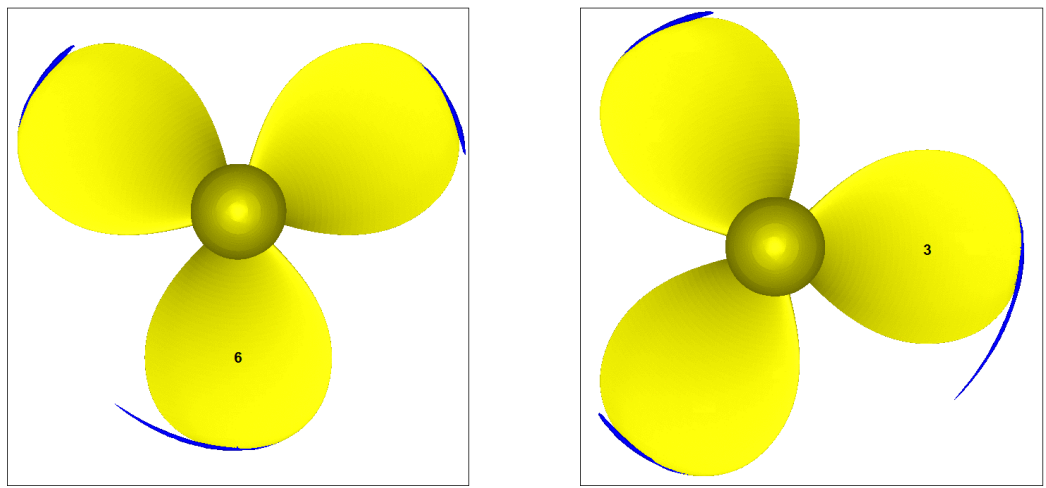

Figure 6.26: Propeller 4119 DTRC. $J=0.833, \operatorname{Re}_{n}=930250, C_{p}=-2.4$ at 6 o'clock (left) and 3 o'clock (right) position, cavitating flow $\sigma=2.4$, G3 mesh

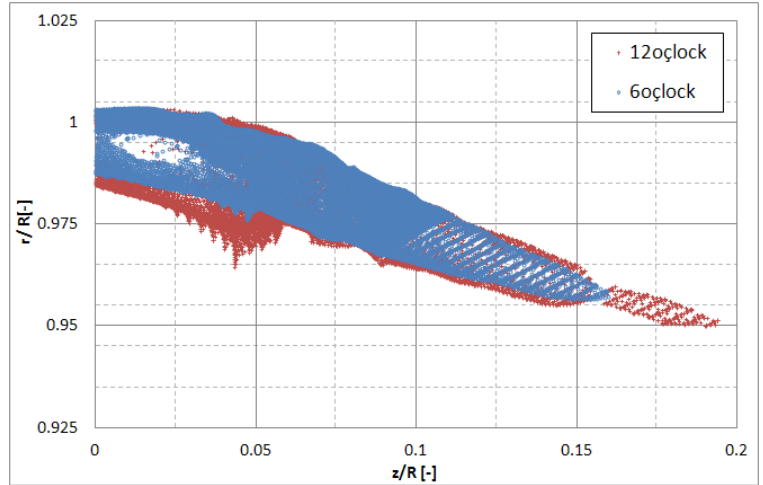

Figure 6.27: Propeller 4119DTRC. $J=0.833, \mathrm{Re}_{n}=930250$, Radial location of the tip vortex core (based on $C_{p}=-2.4$ iso-surface) in downstream direction. Cavitating flow comparison of result for 12 o'clock and 6 o'clock position, G3 mesh 


\subsubsection{Conclusions}

The use of the RANS method to simulate wetted and cavitating flows over a conventional low skew propeller (the 4119 DTRC propeller) has been assessed.

It can be stated that CFD can predict propeller performance within $1 \%$ for thrust and $3 \%$ for torque conforming to the surface grid requirements guidelines and the domain size, solver settings and turbulence modeling described in the beginning of the present section. Using an adequate grid approach, using refinement of the grid in the region of the tip vortex, one can also predict the inception and development of the tip vortex close to the propeller tips.

The far field development of the tip vortex downstream of the wetted propeller is not resolved due to limited grid resolution but also due to the fast decay of the vortex features because of the turbulence modeling (see [89] win which the author shows that the Spalart-Allmaras turbulence model highly over-predicts the turbulence kinetic energy in comparison to the RSM model and [34] in which the authors are testing different turbulence models on the same mesh, concluding that using the two-equation turbulence models the tip vortex decays fast).

Numerical simulations of cavitating flow for the 4119 DTRC open propeller, in terms of prediction of forces, blade surface pressures and velocity field show expected features. But, as in the wetted flow case the cavitating tip vortex decays fast due to insufficient grid resolution and turbulence modeling (as for the wetted flow simulations, see the conclusions found in [34]).

Since the analysed propeller has a uniform inlet and of main interest are the tip vortex inception and its visualisation in terms of downstream development in the close and far field; the current method is considered sufficient adequate for assessing the cavitating tip vortex during the design stage.

\subsection{Leading Edge Skew Propeller}

\subsubsection{Geometry}

The second propeller test case is that of a real designed propeller. This propeller is studied for wetted and cavitating flow both for model-scale and full-scale. Since the ultimate goal of the thesis is the cavitation modelling of full scale real propellers units, the current test case forms the final step of the present thesis.

The investigated propeller geometry, the so-called Leading Edge Skew Pro- 
peller, is a test case used within the Leading Edge EU project, see [66]. Figure 6.28 shows the surface mesh of the model-scale Leading Edge Skew Propeller. The four-bladed propeller has a model scale diameter of $0.2333 \mathrm{~m}$ and the RPM used in the model tests and simulations is RPM=840. The design condition of the Leading Edge skew Propeller is $J=0.6$, which corresponds to an inlet velocity of $V=1.97 \mathrm{~m} / \mathrm{s}$ and a Reynolds number $\operatorname{Re}_{n}=$ $7.62 \times 10^{5}$. General propeller data are given in table 6.6.

\begin{tabular}{|c|c|}
\hline Parameter & \\
\hline Scale Factor & 22.29 \\
\hline Diameter & $0.2333 \mathrm{~m}$ \\
\hline P/D at 0.7R & 1.224 \\
\hline Boss/Diameter ratio & 0.181 \\
\hline Dhub/D & 0.308 \\
\hline Expanded blade area ratio & 0.729 \\
\hline Number of blades & 4 \\
\hline C $(0.7 \mathrm{R}) / \mathrm{D}$ & 0.5 \\
\hline Design J & 0.6 \\
\hline
\end{tabular}

Table 6.6: Geometry of scaled Leading Edge Skew Propeller

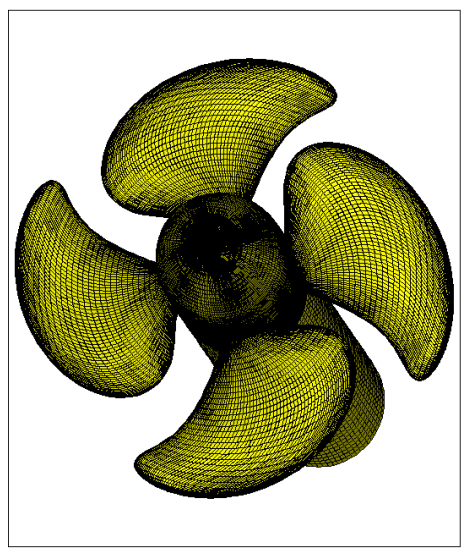

Figure 6.28: Surface mesh Leading Edge Skew Propeller, per blade 8000 cells 


\subsubsection{Computational domain and mesh}

To compute the open water characteristics of the propeller, each blade is meshed based on an O-grid type. The size of the cylindrical domain, in terms of the propeller diameter is: the inlet plane is at $2 \mathrm{D}$ upstream, the outlet plane is at 3D downstream and the radius of the cylinder that forms the far field is $2 \mathrm{D}$. The $y^{+}$values satisfy the requirements for the application of the law of the wall, as recommended in [17].

The average size of the cells of the surface mesh is about $0.012 \mathrm{~m} \times 0.017 \mathrm{~m}$, therefore around the recommended grid size that follows from the Uncertainty Study, chapter 4.

\subsubsection{Setting of the numerical solution parameters}

As for all simulations in this thesis, the choice of the numerical parameters is based on the following approach: RANS equations are solved using the SIMPLE algorithm and the MARS differencing scheme; turbulence model is the two-equation RNG $k-\varepsilon$ turbulence model, see Underling Flow Characteristics Chapter. This is the only investigated turbulence model able to accurately predict drag and cavitation phenomena.

For the rotation of the propeller, the Rotating Reference Frame approach is used which applies to the case of the steady wetted flow, as described in the introduction and in [17]. For the case of cavitating flow, the moving mesh approach is required as discussed in the beginning of the present chapter.

\subsubsection{Results wetted flow}

As for the case of the 4119 DTRC propeller, results have been obtained for various inlet velocities. This provides the open water curves of the propeller performance. Using the thrust and torque coefficient defined in equations 6.3 and 6.4, the performance curves are computed and presented in figure 6.29. In this figure, the results of the numerical simulation for the modelscale Leading Edge Skew Propeller are compared with the measurement data from the report of the Leading Edge project [66].

The agreement between the results of the CFD simulations and the experimental data is satisfactory for the advance ratios considered. The small differences between this open water curves can be related to the laminar flow present on the part of the blades close to the hub. The distribution of $y^{+}$on 


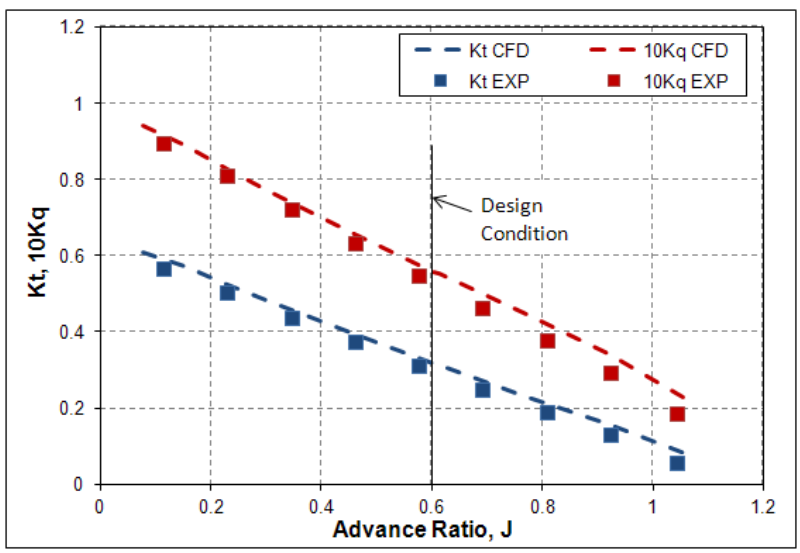

Figure 6.29: Model-scale Leading Edge Skew Propeller. Open Water Curves, $\mathrm{D}=0.2333 \mathrm{~m}, \mathrm{RPM}=840, \mathrm{Re}_{n}=7.62 \times 10^{5}$, Initial grid, wetted flow.

the blade at the design condition $(\mathrm{J}=0.6)$ of the model scale Leading Edge Skew Propeller is plotted in figure 6.30, for both the suction and pressure side. The representative residuals for the conservation of mass, momentum and the equations associated with the turbulence model are presented in figure 6.31. These results are within the requirements of the wall function law $\left(y^{+}\right.$values between 30 and 100) and the requirement for the convergence criterion $\left(10^{-4}\right)$.

\begin{tabular}{|c|c|c|c|}
\hline Design Condition, J=0.6 & Simulation & Measurement & Difference \\
\hline Unit & {$[-]$} & {$[-]$} & {$[\%]$} \\
\hline $\mathrm{Kt}$ & 0.319 & 0.30 & 5.9 \\
\hline $10 \mathrm{Kq}$ & 0.563 & 0.534 & 5.15 \\
\hline
\end{tabular}

Table 6.7: Leading Edge Skew Propeller. Performance at design condition, $\mathrm{J}=0.6, \mathrm{Re}_{n}=7.62 \times 10^{5}$, Initial grid, wetted flow, comparison of results of simulations and measurements

The design condition to be investigate in detail corresponds to advance ratio $J=0.6$. For this condition, the thrust and torque coefficients predicted by CFD are compared with experimental data in table 6.7. The performance is predicted are within $6 \%$ from the measured values and can be considered sufficiently accurate for further study into tip vortex flow. 


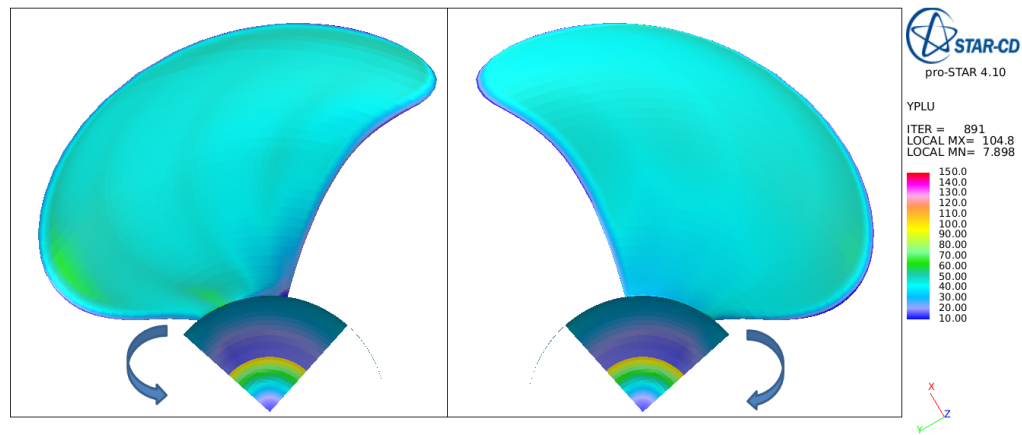

Figure 6.30: Model-scale Leading Edge Skew Propeller. $y^{+}$distribution, $\mathrm{J}=0.6, \mathrm{Re}_{n}=7.62 \times 10^{5}$, on blade suction side (left) and pressure side (right), Initial grid

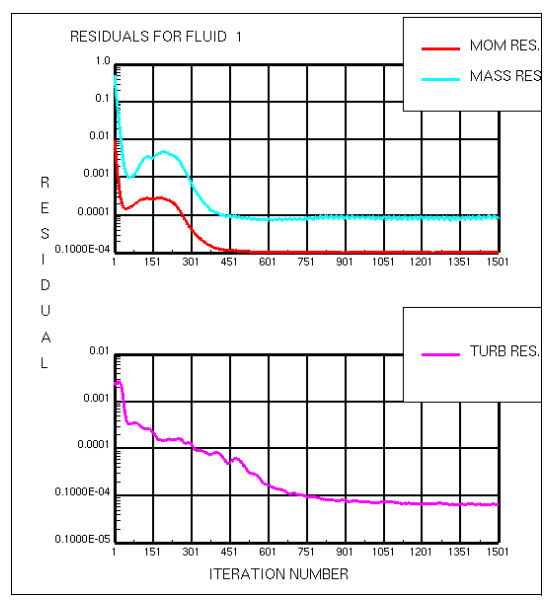

Figure 6.31: Leading Edge Skew Propeller. Residuals for mass, momentum and turbulence equations, $\mathrm{J}=0.6, \mathrm{Re}_{n}=7.62 \times 10^{5}$, Initial grid

At the investigated design condition $(J=0.6)$, the experiments exhibit a cavitating leading edge-tip vortex, see [66]). Therefore a region with low pressures is expected near the blade leading edge as well as in the tip vortex. For this the tip vortex is determined for the wetted flow condition. Consequently the region where the tip vortex appears is refined using the developed Q-factor - Cp method, see the Vortex Detection and Visualisation Chapter. 


\begin{tabular}{|c|c|}
\hline Grid & Number of cells \\
\hline Initial & $2.1 * 10^{6}$ \\
\hline Refined1 & $2.3 * 10^{6}$ \\
\hline Refined2 & $2.5 * 10^{6}$ \\
\hline Refined3 (Final) & $3.0 * 10^{6}$ \\
\hline
\end{tabular}

Table 6.8: Leading Edge Skew Propeller. Number of cells for various grid refinements

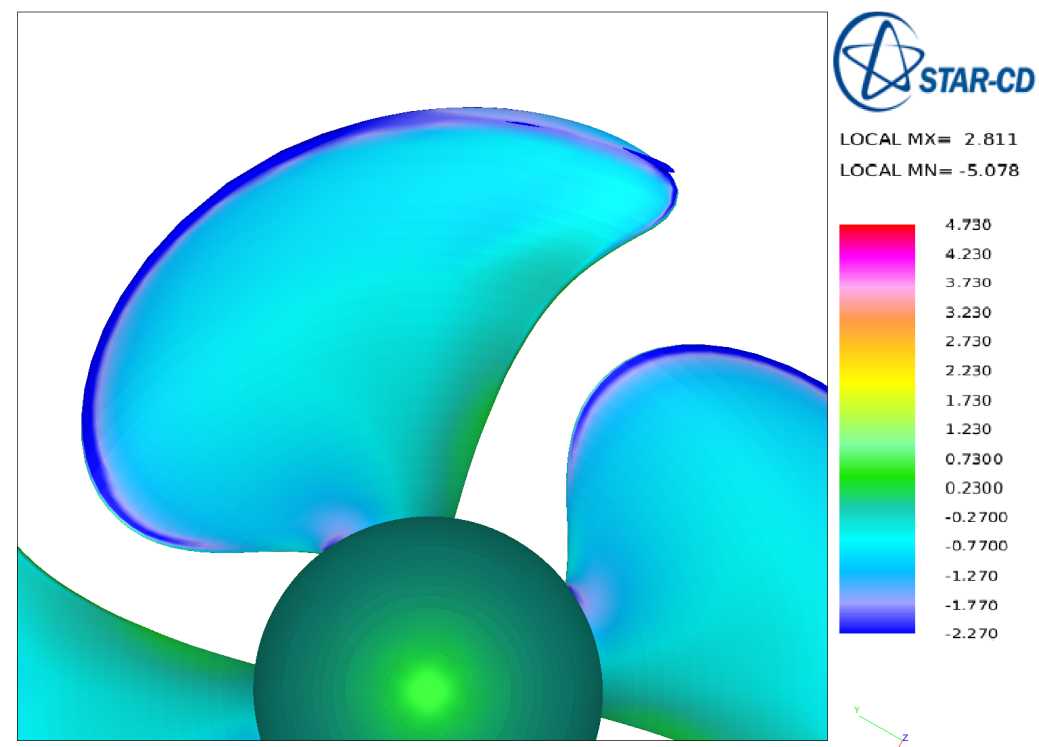

Figure 6.32: Model Scale Leading Edge Skew Propeller, J=0.6, $K_{t}=0.32$, $\operatorname{Re}_{n}=7.62 \times 10^{5}$. Distribution of Cp on the suction side and Cp iso-surface=2.27 , wetted flow. Refined3 grid

This procedure is similar to the one used for the 4119 DTRC propeller. After three steps of the refinement process the number of grid cells reaches its limit of 3 million cells. The number of cells for each refined grid is presented in table 6.8.

The distribution of the pressure coefficient obtained on the blade of the refined grid is shown in figure 6.32. A region with a low pressure coefficient is observed at the leading edge and at the tip of the propeller blade, indicating 


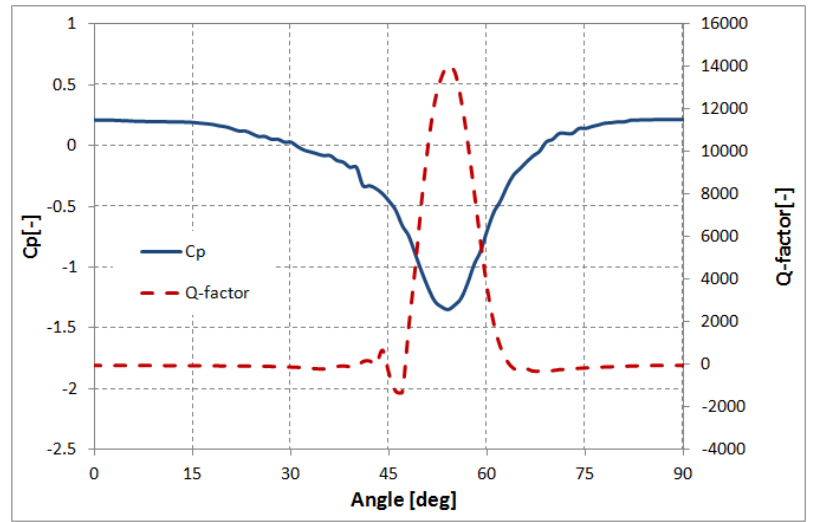

Figure 6.33: Model-scale Leading Edge Skew Propeller. Distribution of pressure coefficient and Q-factor on traverse through the tip vortex core in plane $\mathrm{z}=0.34 \mathrm{R} . \mathrm{J}=0.6, \mathrm{Re}_{n}=7.62 \times 10^{5}$, wetted flow

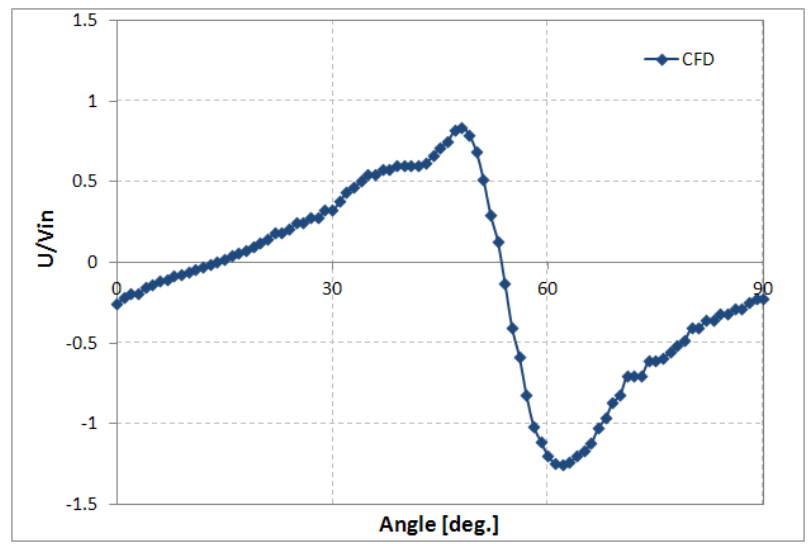

Figure 6.34: Model-scale Leading Edge Skew Propeller. Distribution of azimuthal velocity component along traverse through the tip vortex core in plane $\mathrm{z}=0.34 \mathrm{R}$. $\mathrm{J}=0.6, \mathrm{Re}_{n}=7.62 \times 10^{5}$, wetted flow

the presence of a tip vortex. Figure 6.33 shows the pressure coefficient and $\mathrm{Q}$-factor at a representative location downstream of the propeller $(\mathrm{z}=0.34 \mathrm{R})$. This figure clearly shows the presence of the tip vortex. From this figure, the tip vortex is visible at the location with a low value of the pressure coefficient and a high value of the Q-factor. The corresponding distribution of 


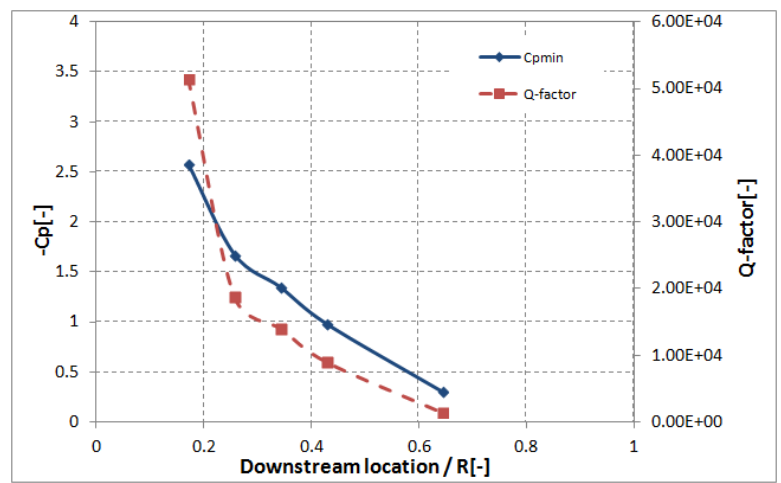

Figure 6.35: Model-scale Leading Edge Skew Propeller. Minimum Cp and maximum Q-factor as function of downstream distance. $\mathrm{J}=0.6, \mathrm{Re}_{n}=7.62 \times$ $10^{5}$, wetted flow

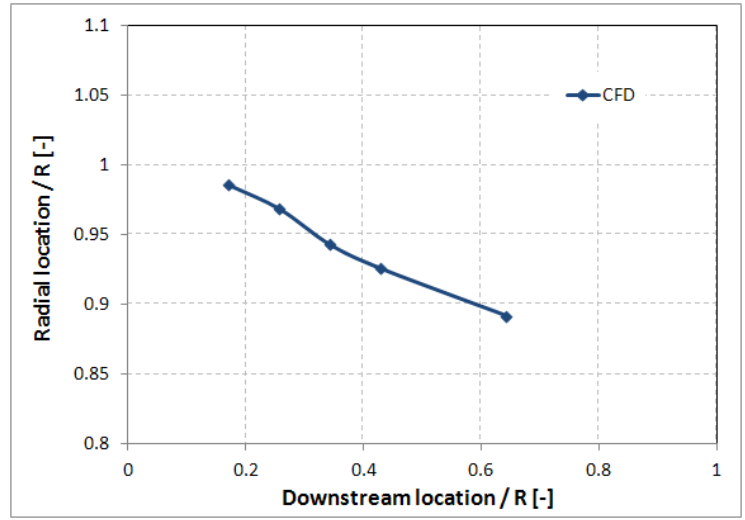

Figure 6.36: Model-scale Leading Edge Skew Propeller. Radial location tip vortex as function of downstream distance. $\mathrm{J}=0.6, \mathrm{Re}_{n}=7.62 \times 10^{5}$, wetted flow

the azimuthal velocity along a traverse through the tip vortex core in the plane $\mathrm{z}=0.34 \mathrm{R}$ downstream of the propeller is plotted in figure 6.34 . This velocity distribution is typical for tip vortex flow, with maximum and minimum values at the edge of the core of the propeller tip vortex. Analysing the variation of the predicted minimum pressure coefficient and the predicted maximum Q-factor as function of downstream distance shows the decay of 
the tip vortex, see figure 6.35. For the Leading Edge Skew Propeller the minimum pressure and maximum Q-factor corresponding to the tip vortex are decaying fast downstream, as also found for the 4119 DTRC propeller. The radial location of the tip vortex at various downstream locations is plotted in figure 6.36 .

The radial location of the tip vortex based on the pressure coefficient isosurface $C_{p}=-2.27$ is plotted in figure 6.37 for the initial grid and for the refined3 grid. From this figure, the location of the minimum pressure at the leading edge (from $0.5 \mathrm{R}$ to $0.95 \mathrm{R}$ ) of the blade and the tip (from $0.95 \mathrm{R}$ to $1.0 \mathrm{R}$ ) is also clear. It is interesting to observe that for the initial grid the low pressure region at the blade leading edge continues similarly into the low pressure region associated with the tip vortex. For the refined grid the minimum pressure within the tip vortex is slightly decoupled and the minimum pressure is not located at the blade surface but is found in the core of the blade leading edge vortex. This effect indicates that a refined grid can predict the detached tip vortex cavitation as found for the case of propeller 4119 DTRC.

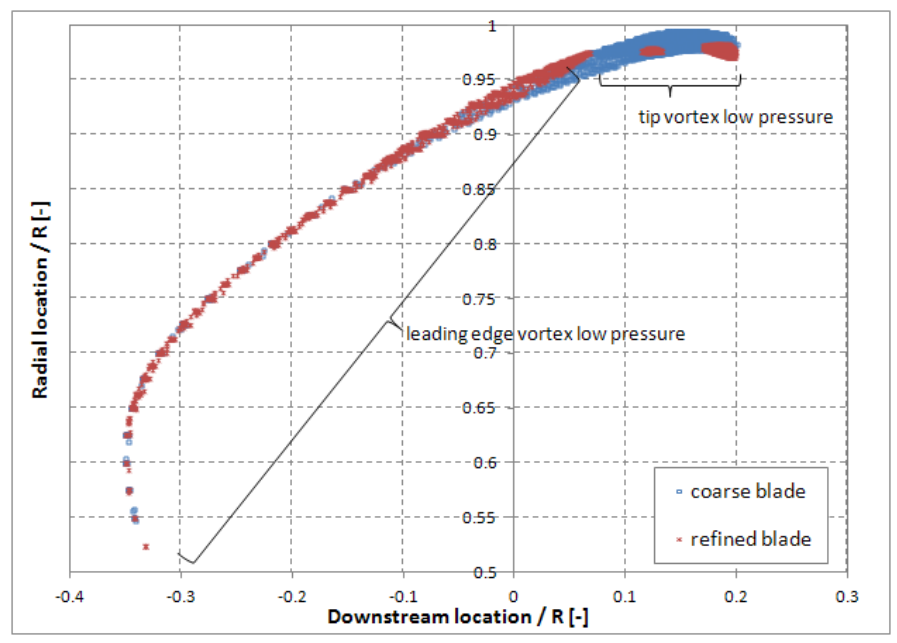

Figure 6.37: Model-scale Leading Edge Skew Propeller. Radial position tip vortex based on $C_{p}=-2.27$ iso-surface. $\mathrm{J}=0.6, \mathrm{Re}_{n}=7.62 \times 10^{5}$, wetted flow, model scale. Initial grid and Refined3 grid 


\subsubsection{Results for cavitating flow}

\section{Inception tip vortex cavitation}

As already demonstrated for the 4119 DTRC propeller, the assessment of the numerically predicted inception of tip vortex cavitation is possible using the results of the wetted flow simulation. For the refined grid (G3) of the Leading Edge Skew Propeller, the minimum pressure coefficient computed for three advance ratios is compared with the inception obtained from experiment ([35]) in figure 6.38. The numerically predicted inception is very close to the measured values (for the leading edge and tip vortex cavitation inception), also taking into account the influence of the water nuclei in the experiments. Once the inception pressure is reached for the wetted flow case, the cavitation is activated and in the following one condition of cavitating flow is studied. Results of that computation are compared with available experimental data.

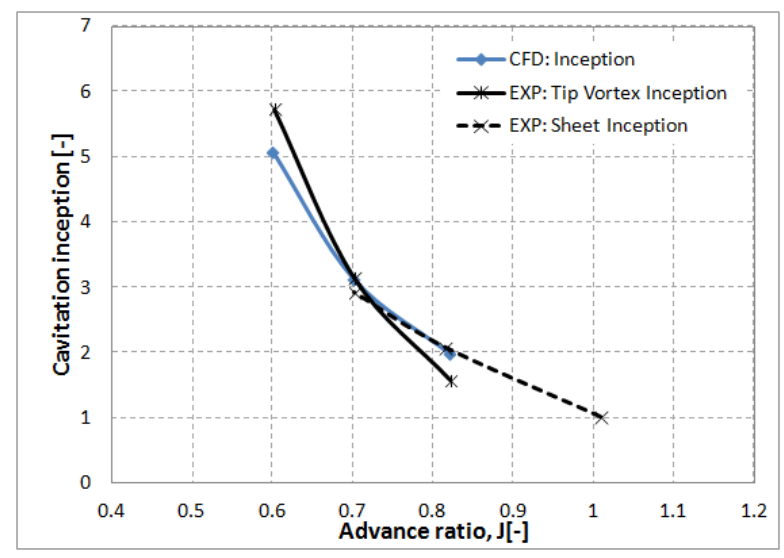

Figure 6.38: Model-scale Leading Edge Skew Propeller. Cavitation inception. Comparison of results of simulations and measurements.

\section{Development tip vortex cavitation}

For the Leading Edge Skew Propeller the development of the cavitating tip vortex is investigated in the present subsection. For this purpose, the outlet pressure is set to $14500 \mathrm{~Pa}$ so that the cavitation number is set to $\sigma_{n}=2.27$ for a thrust coefficient around $k_{t}=0.32$ (as in the homogeneous inflow experiment of the HSVA report [35]). The cavitation number is defined as: 


$$
\sigma_{n}=\frac{p_{\infty}-p_{v}}{\frac{1}{2} \rho(n D)^{2}}
$$

As for previous cases with tip vortex cavitation, the forces are monitored in order to check convergence of the numerical simulation. In figure 6.39, the thrust and torque coefficients are presented for the cavitating condition. In table 6.9, the thrust coefficient is given for wetted and cavitating flow (average value). From analysing these results it can be concluded that when cavitation is present on the Leading Edge Skew Propeller blades, the thrust coefficient increases, in the measurement as well as in the numerical simulations. This is an expected result (previously also found for the Elliptic 11 Rake Hydrofoil and the 4119 DTRC propeller) since the extent of the leadingedge cavity is slightly larger in cavitating conditions $(6.25 \%$ in the simulation and $8.85 \%$ in experiments). Note that compared to the measurement, the numerical simulation predicts slightly higher thrust for both wetted and cavitating flow conditions.

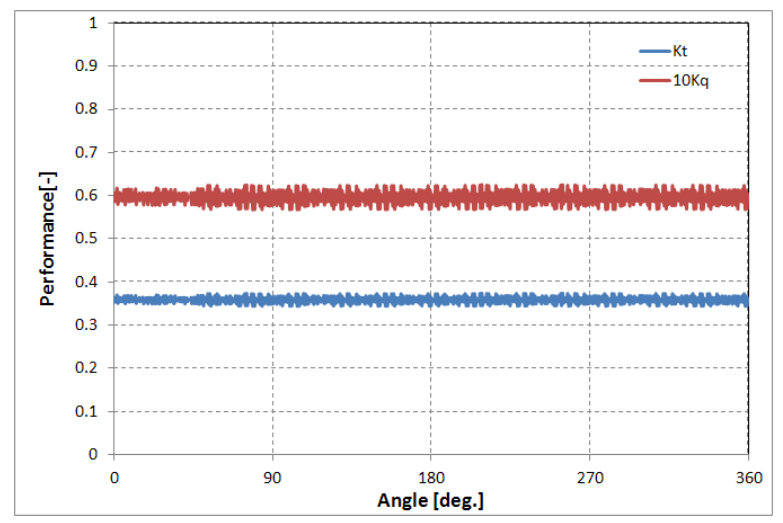

Figure 6.39: Model Scale Leading Edge Skew Propeller. Thrust and torque coefficients, $\mathrm{J}=0.6, \mathrm{Re}_{n}=762000, \sigma_{n}=2.27$, cavitating flow

\begin{tabular}{|c|c|c|c|}
\hline Kt & Wetted & Cavitating & Difference [\%] \\
\hline Simulation & 0.319 & 0.35 & 8.85 \\
\hline Measurement & 0.30 & 0.32 & 6.25 \\
\hline
\end{tabular}

Table 6.9: Model Scale Leading Edge Skew Propeller. Thrust coefficient for wetted and cavitating conditions. $\mathrm{J}=0.6, \mathrm{Re}_{n}=762000$. 
The distribution of the pressure coefficient and the iso-surface $C_{p}=-2.27$, for cavitating flow is shown in figure 6.40. Compared to the pressure distribution for wetted flow, see figure 6.32 , the pressure distribution for cavitating flow $\left(\sigma_{n}=2.27\right)$ shows a tip vortex as expected from the previous tip vortex test cases.

Figure 6.41 shows the predicted pressure iso-surface for $C_{p}$ equal to the vapor pressure coefficient $\left(C_{p}=-2.27\right)$. Figure 6.42 shows the cavitation observed in the experiment at HSVA [35].

Comparing figure 6.41 and figure 6.42 , it is clear that the numerical simulations are able to capture the location of the leading edge-tip vortex cavitation as observed in the measurements, for the same cavitation number and thrust coefficient. Note that in the results of the numerical simulation there is a well-defined tip vortex though it does decay already relatively close to the blade. Without refining the grid the tip vortex region the results would not have shown this vortex, e.g. see the results in [77].

The influence of cavitation on the distribution of the pressure coefficient

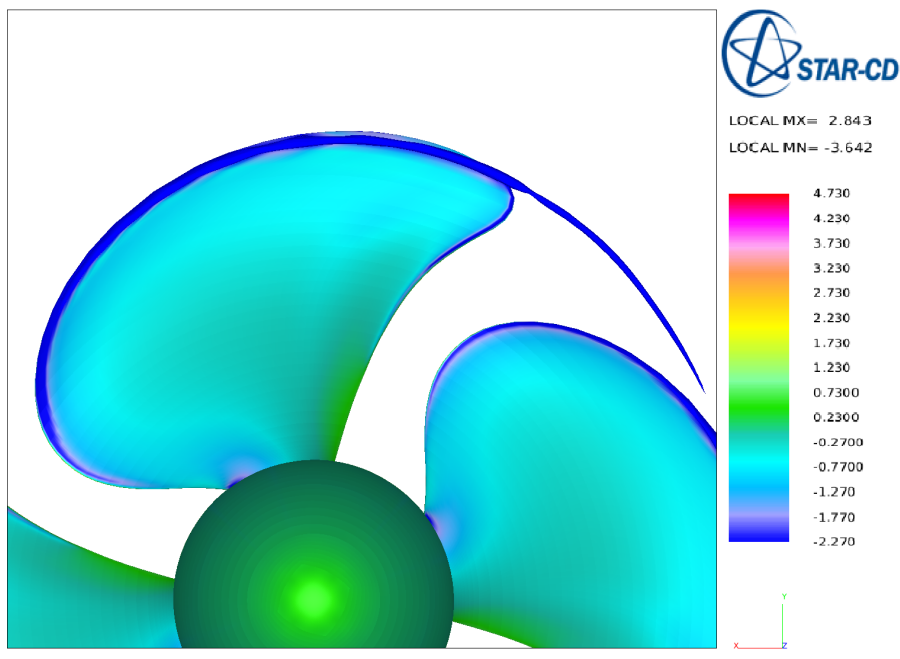

Figure 6.40: Model Scale Leading Edge Skew Propeller. Distribution of suction side surface $\mathrm{Cp}$ and $\mathrm{Cp}$ iso-surface $C_{p}=-2.27, \mathrm{~J}=0.6, \mathrm{Re}_{n}=762000$, $\sigma_{n}=2.27$, cavitating flow

and on the azimuthal component of the velocity in the vortex core, downstream of propeller at the Leading Edge Skew Propeller, are analysed in the following. 


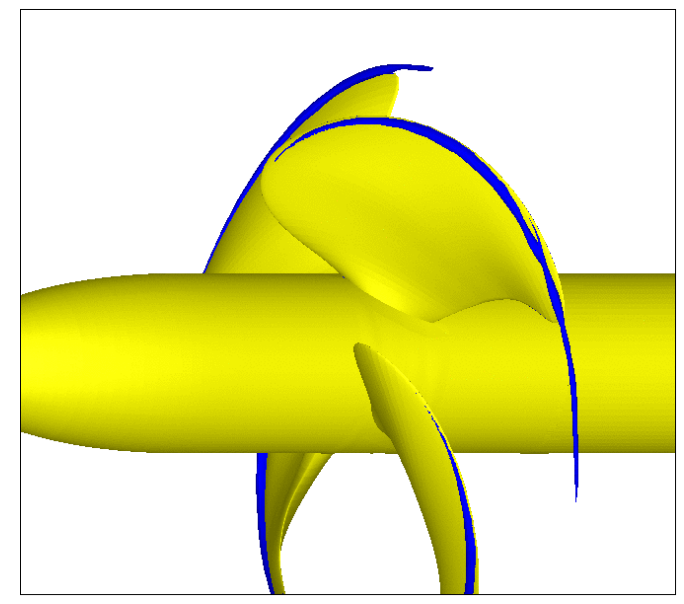

Figure 6.41: Model-scale Leading Edge Skew Propeller. Iso-surface of $C_{p}=$ $-2.27, \mathrm{~J}=0.6, \mathrm{Re}_{n}=762000, \sigma_{n}=2.27$, cavitating flow

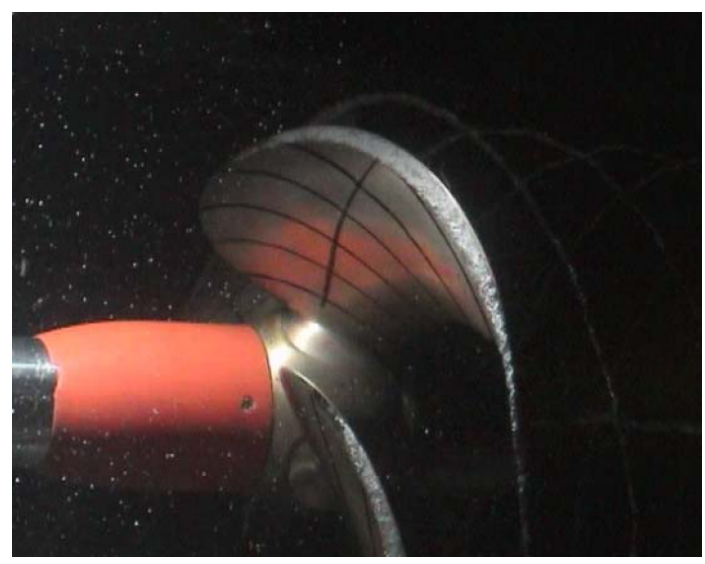

Figure 6.42: Model-scale Leading Edge Skew Propeller, Visualisation cavitation in experiment [35], $\mathrm{J}=0.6, \mathrm{Re}_{n}=762000$

Figure 6.43 presents the distribution of the pressure coefficient along a traverse through the vortex core in the plane $\mathrm{z}=0.34 \mathrm{R}$, for wetted and cavitating 


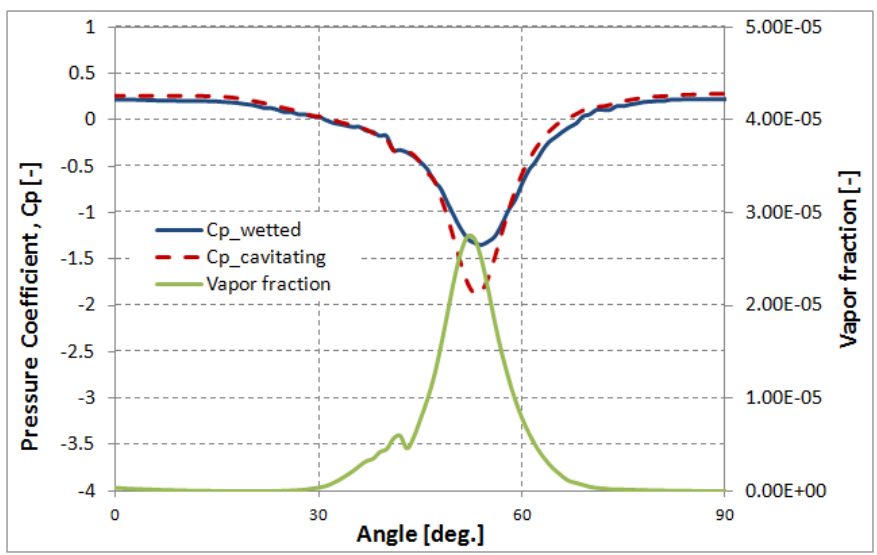

Figure 6.43: Model scale Leading Edge Skew Propeller. Distribution of Cp along traverse through vortex core in plane $\mathrm{z}=0.34 \mathrm{R}$. Also shown is the vapor fraction, $J=0.6, \operatorname{Re}_{n}=762000$, wetted and cavitating flow $\left(\sigma_{n}=2.27\right)$

flow. Also shown is the distribution of the vapor content (cavitating case). Figure 6.44, shows the distribution of the azimuthal velocity along the same traverse. In cavitating flow the pressure in the tip vortex core is lower and the azimuthal velocity is larger. Though the circulation appears to be the same, the velocity in the center of the core is higher in case of cavitating flow. This was also the case for the Elliptic 11 rake hydrofoil and 4119 DTRC propeller. In figure 6.45 the radial location of the pressure coefficient $C_{p}=-2.27$ isosurface is plotted for both the wetted and the cavitating flow condition. The cavitating leading edge is slightly thicker when the tip vortex cavitates and for cavitating flow the downstream extension of the vortex is longer than for wetted flow.

Note that this case is also a test case with a uniform inflow. For this case the leading edge-tip vortex is very stable during a revolution of propeller and the only oscillation is due to the the hydrostatic pressure. In figures 6.46 and 6.47 , results are shown for four blade positions. There is a slight difference in the length of the tip vortex of the 4 blades due to only one of the blades having a refined grid in the region of the tip vortex. For a better view of the vortex and roll-up, figure 6.48 compares the radial position of the tip vortex for the 12 o'clock position and the 6 o'clock position of the blade.

Further conclusions on the radiated pressures from the tip vortex are not appropriate for the current case for which the oscillations that are present are mainly blade frequency related due effect of the hydrostatic pressure. 


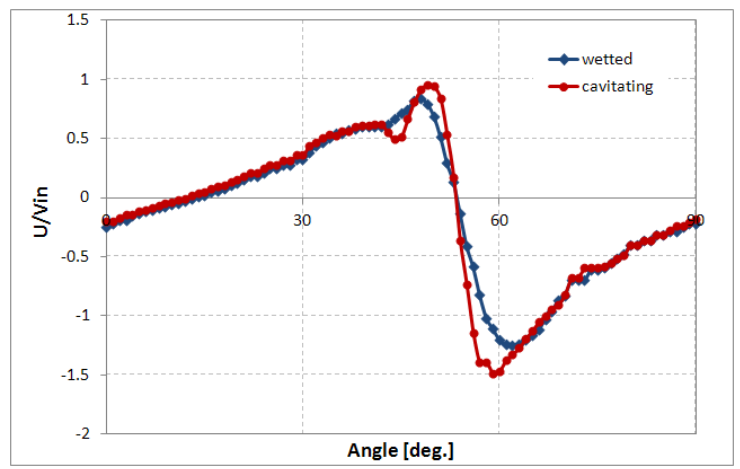

Figure 6.44: Model scale Leading Edge Skew Propeller. Distribution of azimuthal velocity along traverse through vortex core in plane $\mathrm{z}=0.34 \mathrm{R}$, $\mathrm{J}=0.6, \mathrm{Re}_{n}=762000$. Comparison of results of wetted flow and cavitating flow $\left(\sigma_{n}=2.27\right)$.

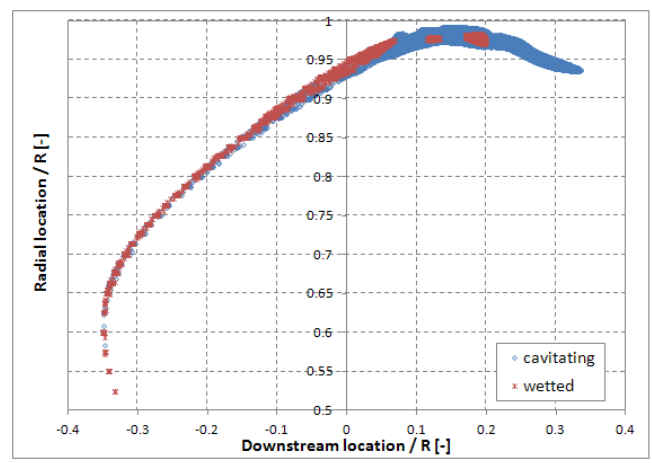

Figure 6.45: Model scale Leading Edge Skew Propeller. Radial location of leading edge-tip vortex, determined as location of minimum value of $C_{p}$ as function of downstream distance, results for wetted flow compared with results for cavitating flow $\left(\sigma_{n}=2.27\right), \mathrm{J}=0.6, \mathrm{Re}_{n}=762000$ 


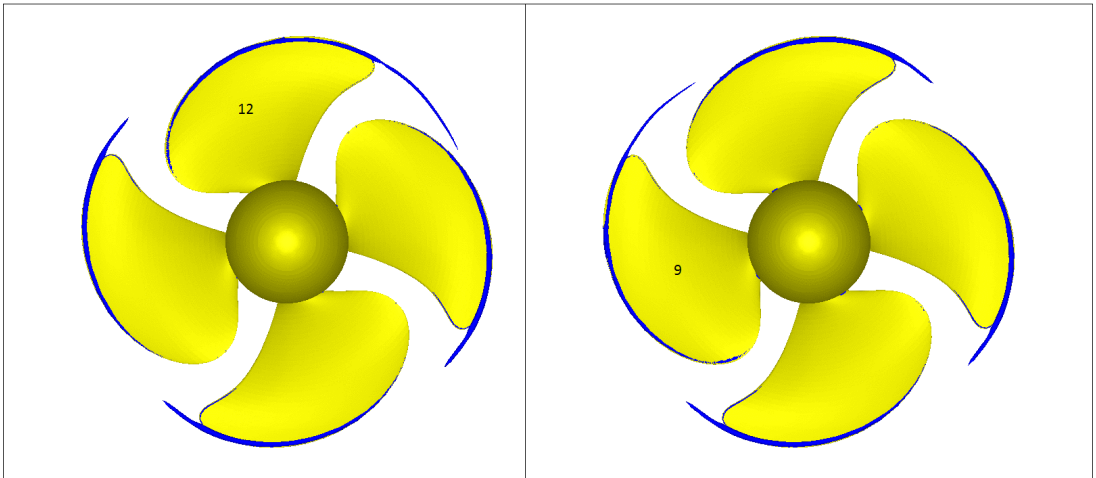

Figure 6.46: Model-scale Leading Edge Skew Propeller. $C_{p}=-2.27$ isosurface, $\mathrm{J}=0.6, \mathrm{Re}_{n}=762000, \sigma_{n}=2.27,12$ o'clock (left) and 9 o'clock (right) position, blade refinement in the tip vortex

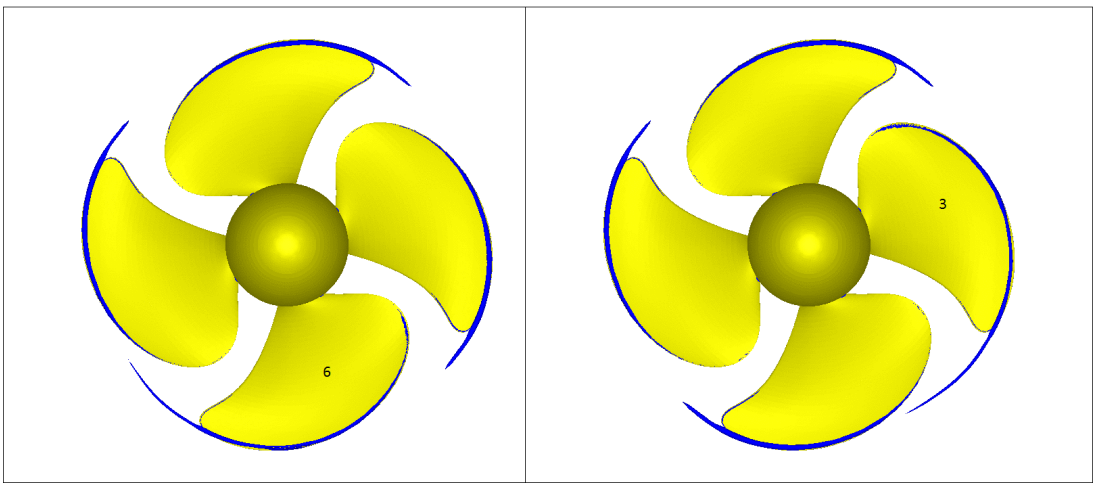

Figure 6.47: Model-scale Leading Edge Skew Propeller. $C_{p}=-2.27$ isosurface, $\mathrm{J}=0.6, \mathrm{Re}_{n}=762000, \sigma_{n}=2.27,6$ o'clock (left) and 3 o'clock (right) position, blade refinement in the tip vortex 


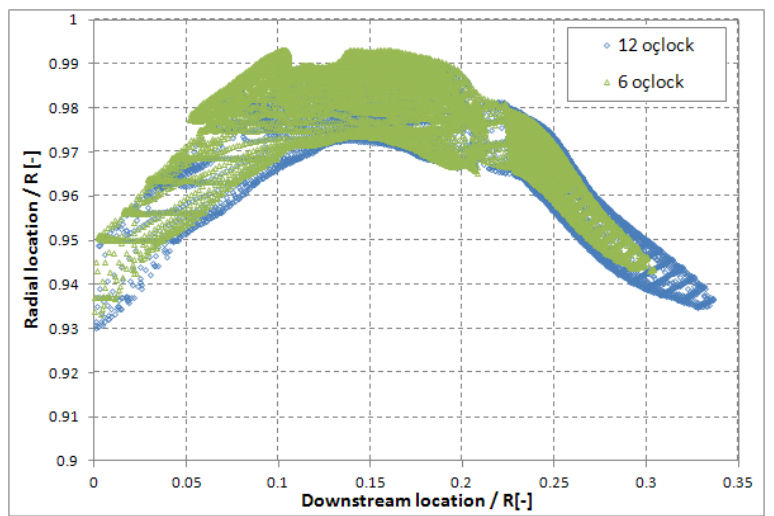

Figure 6.48: Model-scale Leading Edge Skew Propeller. Radial location of leading edge-tip vortex based on location of minimum $C_{p}$ as a function of downstream location. Shown are the results for the 12 o'clock and 6 o'clock position of the blade eith refined grid, $\mathrm{J}=0.6, \mathrm{Re}_{n}=762000, \sigma_{n}=2.27$

\subsubsection{Scaling}

The main issue when predicting full-scale tip vortex cavitation inception employing model test experiments is the scaling. As summarised by ITTC (see $[2]$ ), the scale effects are mainly due to the effects due to differences in the Reynolds number, the bubble nuclei and the hydrofoil lift.

In 1962, based on a series of experiments on hydrofoils tested in water tunnels, McCormick (see [54]) observed that the cavitation number at which the inception of the tip vortex cavitation occurs increases with Reynolds number. The scaling rule proposed by McCormick for tip vortex cavitation inception is expressed as:

$$
\frac{\sigma_{F S}}{\sigma_{M S}}=\left(\frac{R e_{F S}}{R e_{M S}}\right)^{n}
$$

In equation $6.11 n=0.35$. However, within the literature this value varies from 0.25 to 0.4 , depending on the facility in which the model test experiment has been performed. Also, the value of $n$ is related to the nuclei content of the water in the basin. When the propeller Reynolds number is defined as in equation 6.1, the full-scale Reynolds number of the Leading Edge Skew Propeller is $\operatorname{Re}_{F S}=6.3 * 10^{7}$. Note that in equation 6.11, the FS and MS subscript refer to full-scale and model-scale, respectively.

In numerical simulations, the scaling of nuclei, hydrofoil lift and geometry 
is not an issue. Since in numerical simulations, inception can be analysed employing results for wetted flows, only the effect of Reynolds number can be assessed numerically. In the following, the results of the numerical simulations for wetted flow around the full-scale Leading Edge Skew Propeller will be analysed in terms of the pressure coefficient.

Open water simulations have been performed for the full scale propeller for $R P M=140$ and propeller diameter $D=5.2 \mathrm{~m}$. In figure 6.49 , the scaling of the performance curves for the Leading Edge Skew Propeller are presented. For the Leading Edge Skew Propeller the numerical simulations predict that the torque coefficient stays almost the same, while the thrust coefficient increases with $\operatorname{Re}_{n}$. Therefore the efficiency increases with $\operatorname{Re}_{n}$. This kind of scaling of propeller performance agrees with the ITTC'78 scaling rule, see [4].

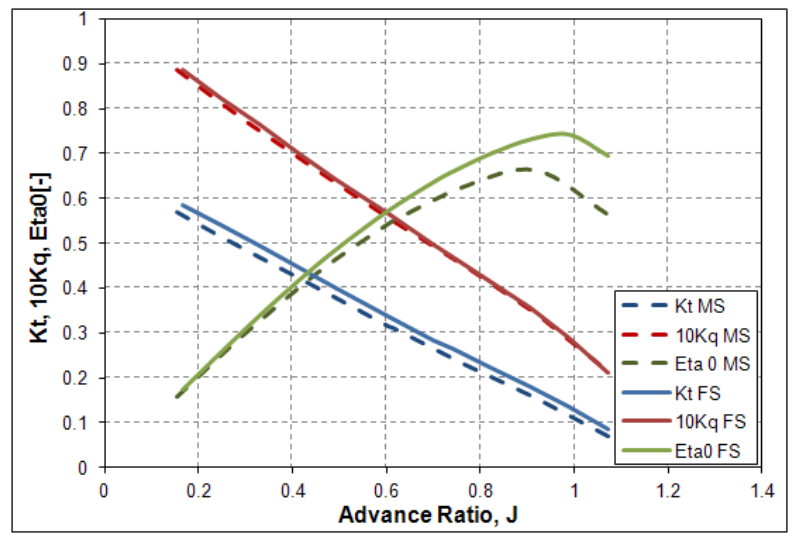

Figure 6.49: Leading Edge Skew Propeller. Open Water Performance Curves, wetted flow, FS: $\operatorname{Re}_{n}=63 * 10^{6}, \mathrm{MS}: \mathrm{Re}_{n}=0.762 * 10^{6}$

For the design condition $(J=0.6)$, the effects of scaling for the Leading Edge Skew propeller performance, inception and tip vortex flow are investigated in more detail. In table 6.10, the conditions for the model-scale and full-scale test cases are summarised.

For the analysed condition the convergence history of the residuals is presented in figure 6.50. Figure 6.51 shows the distribution of $y^{+}$on the propeller suction and pressure side. The residuals and the values of $y^{+}$agree with the results of the previous simulations and conform to the guidelines. 


\begin{tabular}{|c|l|l|l|l|}
\hline & $\begin{array}{l}\text { Diameter, } \\
\mathrm{D}\end{array}$ & $\begin{array}{l}\text { Advance } \\
\text { ratio, J }\end{array}$ & $\begin{array}{l}\text { Inlet veloc- } \\
\text { ity, V }\end{array}$ & $\begin{array}{l}\text { Reynolds } \\
\text { number, } \\
\operatorname{Re}_{n}\end{array}$ \\
\hline & {$[\mathrm{m}]$} & {$[-]$} & {$[\mathrm{m} / \mathrm{s}]$} & {$[-]$} \\
\hline Model Scale (MS) & 0.2333 & 0.6 & 1.97 & $0.762 * 10^{6}$ \\
\hline Full Scale (FS) & 5.2 & 0.6 & 7.31 & $63 * 10^{6}$ \\
\hline
\end{tabular}

Table 6.10: Leading Edge Skew Propeller, model-scale and full-scale conditions, $\mathrm{J}=0.6$

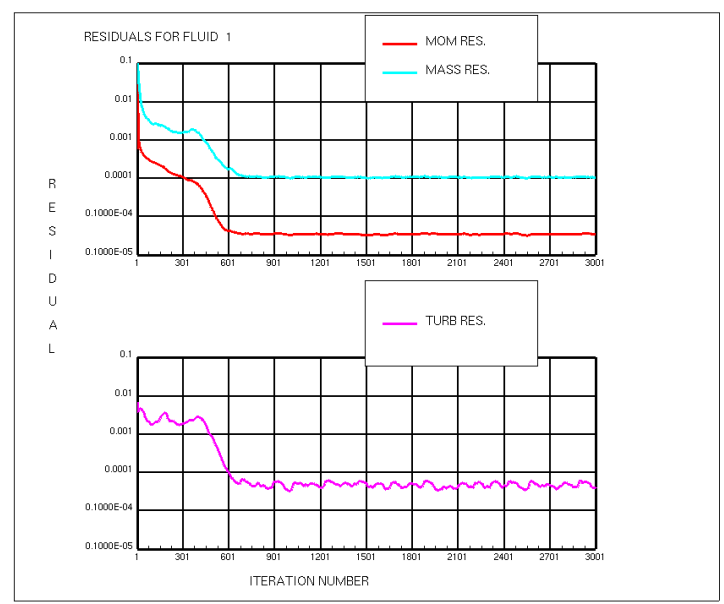

Figure 6.50: Full-scale Leading Edge Skew Propeller. Residuals of mass, momentum and turbulence equations. $\mathrm{J}=0.6, \mathrm{Re}_{n}=6.3 * 10^{7}$, wetted flow

Figure 6.49 gives the performance of the propeller on model-scale and fullscale. Table 6.11 summarizes the thrust and torque coefficients for the analysed condition $(\mathrm{J}=0.6)$. The scaling of the performance of the Leading Edge Skew Propeller derived from the results of the numerical simulations given in table 6.11 predicts that the thrust coefficient at full scale increases with $7.4 \%$, while the torque coefficient increases with just $1.9 \%$ compared to the coefficients for model-scale.

The effect of propeller scale on the tip vortex parameters, like: pressure, azimuthal velocity and Q-factor, are investigated for the wetted flow conditions. We consider the plane at $\mathrm{z}=0.34 \mathrm{R}$, downstream of the Leading Edge 


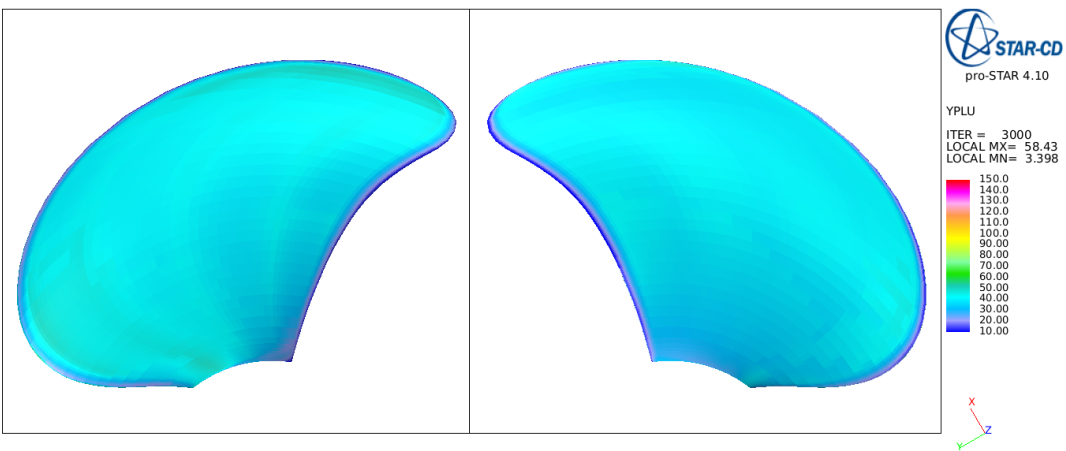

Figure 6.51: Full-scale Leading Edge Skew Propeller. Distribution of $y^{+}$on blade surface, $\mathrm{J}=0.6, \mathrm{Re}_{n}=6.3 * 10^{7}$, wetted flow, suction side (left) and pressure side (right)

\begin{tabular}{|c|c|c|}
\hline & Thrust coefficient, Kt [-] & Torque coefficient, 10Kq [-] \\
\hline Model Scale (MS) & 0.319 & 0.536 \\
\hline Full Scale (FS) & 0.343 & 0.574 \\
\hline
\end{tabular}

Table 6.11: Leading Edge Skew Propeller. Scaling of thrust and torque coefficient. J=0.6, FS: $\operatorname{Re}_{n}=6.3 * 10^{7}$, MS: $\mathrm{Re}=0.762 * 10^{6}$

Skew Propeller.

The distribution of pressure and Q-factor along a traverse through the tip vortex core, are presented in figure 6.52. The azimuthal velocity is presented in figure 6.53. For the full-scale propeller, the distribution of the pressure coefficient, Q-factor and the velocity are similar to the ones for the modelscale propeller, i.e. in the same range, see also figures 6.33 and 6.34. The Q-factor for the full-scale propeller is slightly higher than the model scale correspondent. This indicates that in the numerical simulation tip vortex is slightly stronger at full scale than at the model scale in terms of the Q-factor.

Distribution of surface pressure coefficient and the iso-surface $C_{p}=-2.27$ are shown in figure 6.54. The distribution of the pressure coefficient on modelscale (see figure 6.32), is similar to the one on full-scale while the minimum pressure coefficient on full-scale is lower than that on model scale due the higher Reynolds number.

Analysing the scaling of the minimum pressure coefficient for three advance ratios $(0.6,0.7$ and 0.82$)$ the Reynolds number scaling of the vortex cavitation 


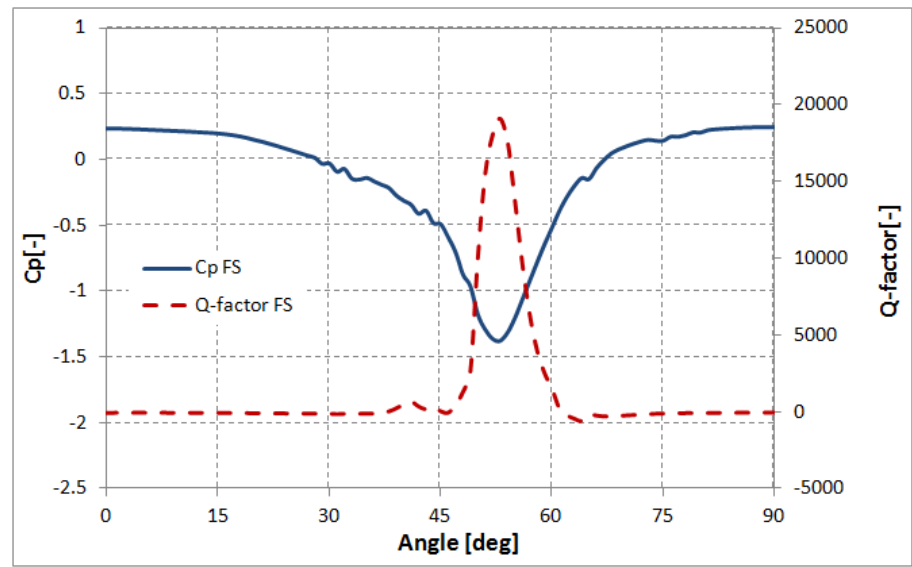

Figure 6.52: Full-scale Leading Edge Skew Propeller in wetted flow. Distribution of $C_{p}$ and Q-factor along a traverse through tip vortex location in plane $\mathrm{z}=0.34 \mathrm{R}$. $\mathrm{J}=0.6, \mathrm{Re}_{n}=6.3 * 10^{7}$

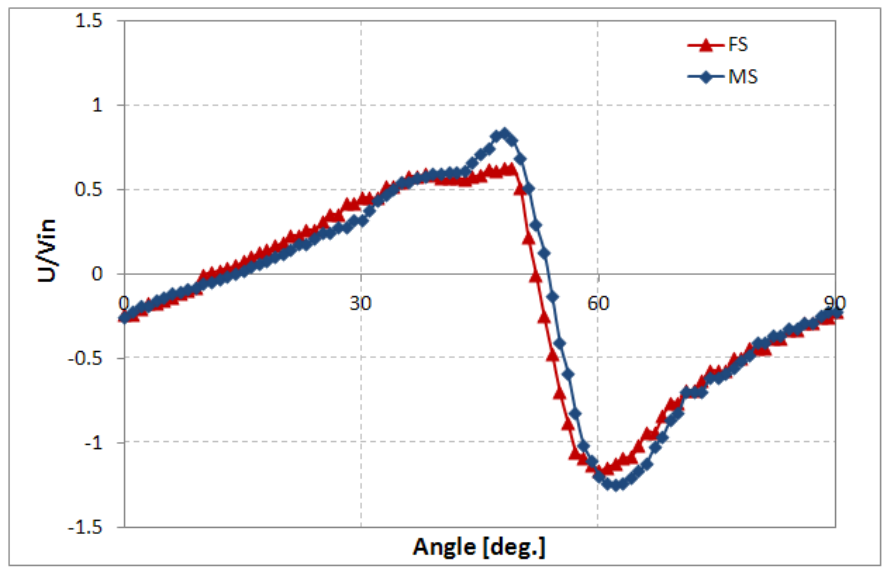

Figure 6.53: Leading Edge Skew Propeller. Distribution of azimuthal velocity along traverse through tip vortex in plane $\mathrm{z}=0.34 \mathrm{R}$. $\mathrm{J}=0.6$, wetted flow. Comparison of results for model scale and full scale, MS: $\operatorname{Re}_{n}=0.762 * 10^{6}$ and FS: $\operatorname{Re}_{n}=6.3 * 10^{7}$

inception can be assessed. As for the 4119 DTRC Propeller, the minimum pressure coefficient for wetted flow conditions is used to assess numerical 


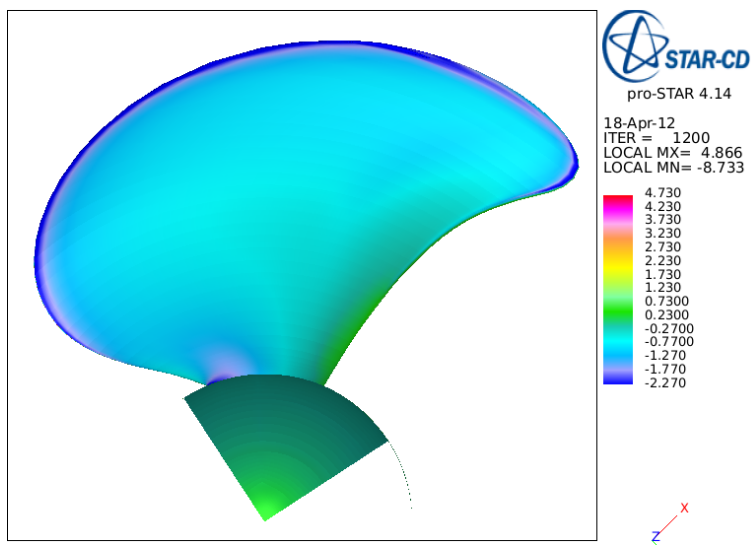

Figure 6.54: Full-scale Leading Edge Skew Propeller. Distribution of surface $C_{p}$ on blade suction side and iso-surface $C_{p}=-2.29$. $\mathrm{J}=0.6, \mathrm{Re}_{n}=6.3 * 10^{7}$, wetted flow

simulation inception values, in figure 6.55. Figure 6.55 shows the numerical simulation values for the minimum pressure coefficient at model-scale and full-scale in comparison to the model test inception values and McCormick scaled inception values, for the Leading Edge Skew Propeller. For $J=0.82$, the CFD results show good agreement with the McCormick's scaling rule. As defined in equation $6.11, \mathrm{n}=0.2$ is predicted, see also [14], compared to $\mathrm{n}=0.25$ up to 0.4 found in the model-tests scaling as from [76]. However, for lower advance ratios the deviations are increased. This can be explained by the observation that for the lower advance ratios the cavitation is not purely tip vortex cavitation but more leading edge-tip vortex cavitation. Thus for the advance ratio $\mathrm{J}=0.6$, the inception rule employing the minimum value of $C_{p}$ does not give satisfactory results. Still the minimum pressure coefficient found in the numerical simulations is well below the cavitation value of $C_{p}=-\sigma=-2.27$. Therefore the results for cavitating flow should not be influenced since the cavitation starts at a much lower value of the cavitation number. 


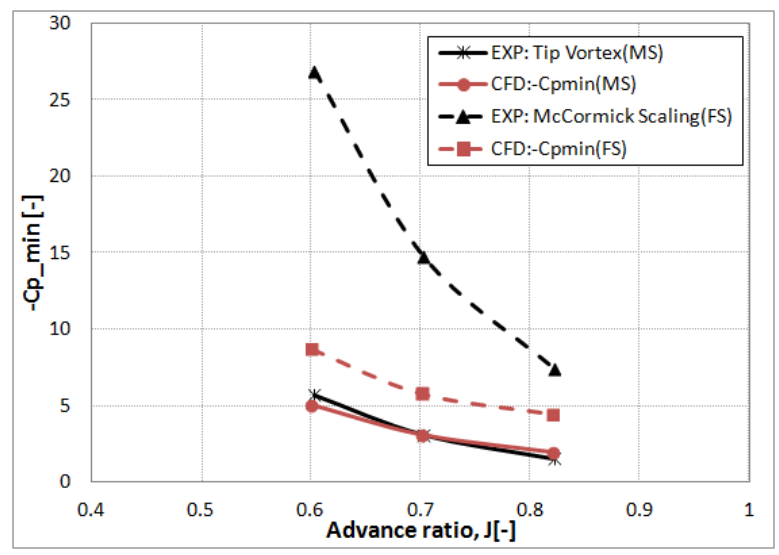

Figure 6.55: Leading Edge Skew Propeller. Scaling of cavitation inception, wetted flow

The convergence of the numerical procedure for the full-scale Leading Edge Skew Propeller is monitored through the variation of the thrust and torque coefficients as a function of angle of rotation during one revolution of the propeller, see figure 6.56. This figure shows a typical variation of the thrust and torque coefficient in cavitating condition, with small oscillations around a mean value. In table 6.12 the mean thrust and torque coefficient are summarized and results for cavitating flow are compared to the ones for wetted flow. This table shows that the full scale thrust and torque coefficients are slightly higher than the ones for wetted flow, as expected.

\begin{tabular}{|c|c|c|}
\hline & Thrust coefficient, Kt [-] & Torque coefficient, 10Kq [-] \\
\hline Full Scale (FS) wetted & 0.343 & 0.574 \\
\hline Full Scale (FS) cavitating & 0.375 & 0.614 \\
\hline
\end{tabular}

Table 6.12: Full-scale Leading Edge Skew Propeller in wetted and cavitating flow $(\sigma=2.27)$. Thrust and torque coefficient. $\mathrm{J}=0.6, \mathrm{Re}_{n}=6.3 * 10^{7}$

Figure 6.57 presents the distribution of the pressure coefficient and vapor fraction along a traverse through the vortex core in the plane $\mathrm{z}=0.34 \mathrm{R}$ downstream of the full scale Leading Edge Skew Propeller in cavitating flow. Figure 6.58 gives the distribution of the azimuthal velocity along the same traverse. As for the case of wetted flow, the pressure distribution and velocity distribution are similar to the ones for cavitating flow on model scale while 


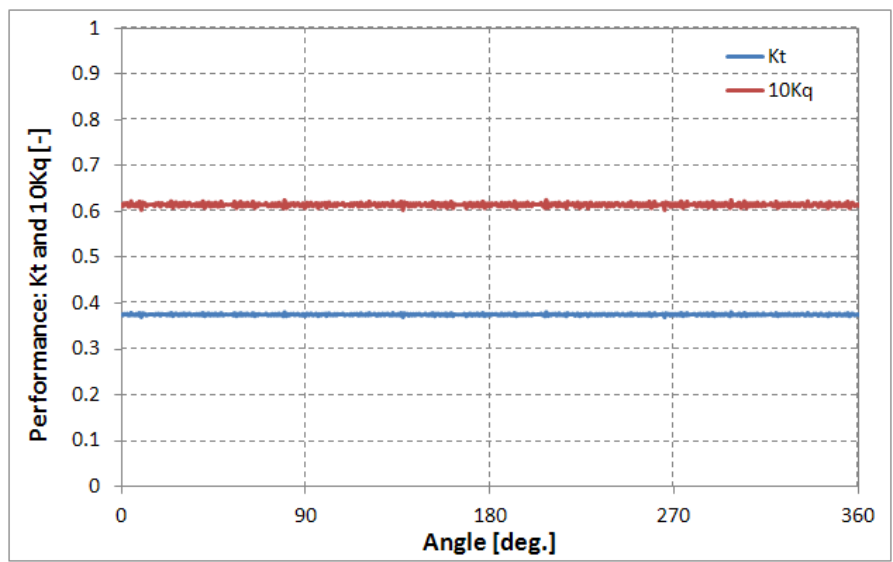

Figure 6.56: Full-scale Leading Edge Skew Propeller in cavitating flow ( $\sigma=$ 2.27). Variation of thrust and torque coefficient during one revolution of propeller. $\mathrm{J}=0.6, \mathrm{Re}_{n}=6.3 * 10^{7}$

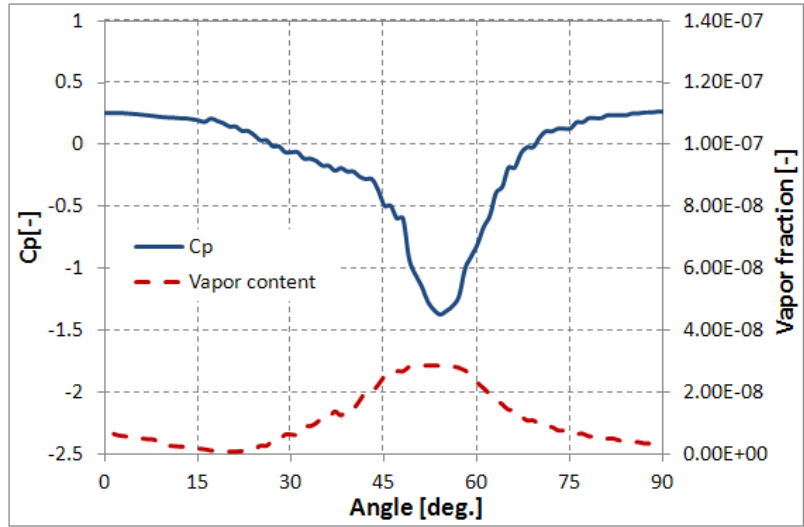

Figure 6.57: Full-scale Leading Edge Skew Propeller in cavitating flow ( $\sigma=$ $2.27)$. Distribution of pressure coefficient and vapor fraction along a circular arc through the tip vortex core in the plane $\mathrm{z}=0.34 \mathrm{R}, \mathrm{J}=0.6, \mathrm{Re}_{n}=6.3 * 10^{7}$

the vapor content is much lower for the full-scale case.

Figure 6.59 shows the distribution of the surface pressure coefficient on the suction side of the propeller and the corresponding iso-surface $\mathrm{Cp}=-\sigma_{n}=-$ 2.27. Comparing this figure to the one for the model scale propeller in figure 


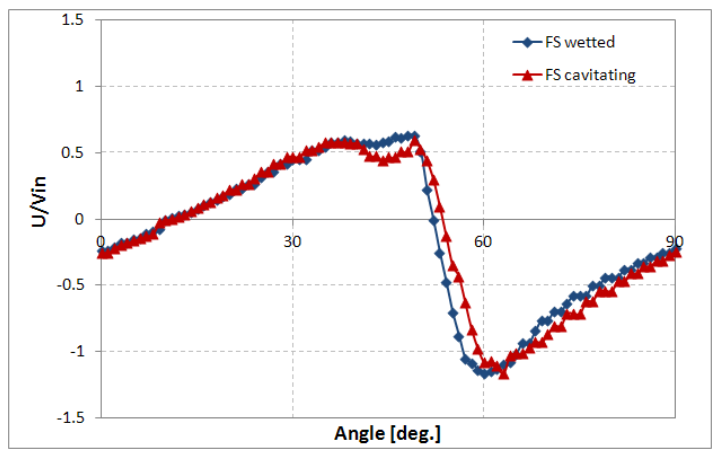

Figure 6.58: Full-scale Leading Edge Skew Propeller in wetted and cavitating flow $(\sigma=2.27)$. Distribution of azimuthal velocity along circular arc through the tip vortex core in plane $\mathrm{z}=0.34 \mathrm{R}$. $\mathrm{J}=0.6, \mathrm{Re}_{n}=6.3 * 10^{7}$

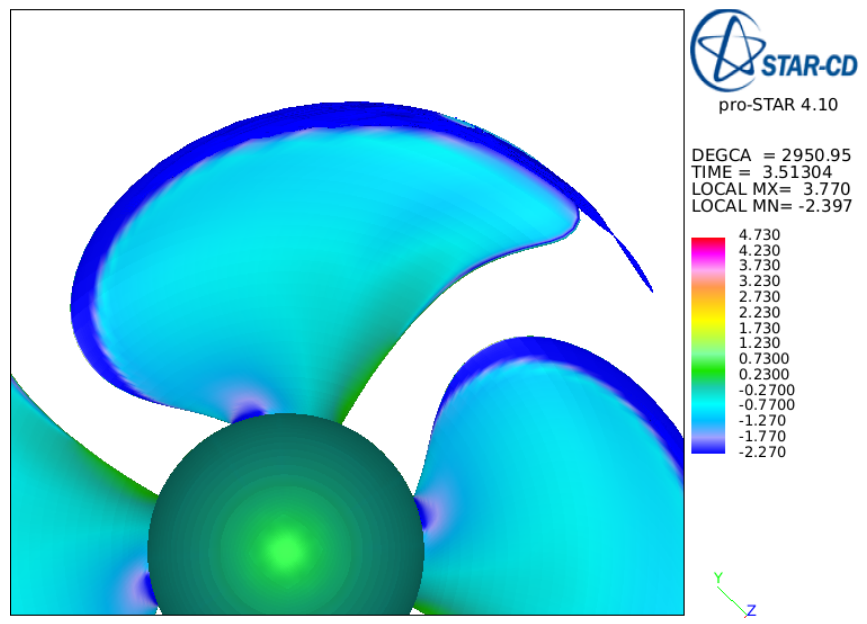

Figure 6.59: Full-scale Leading Edge Skew Propeller in cavitating flow $(\sigma=$ 2.27). Distribution of surface pressure coefficient and iso-surface $C_{p}=-2.27$, $\mathrm{J}=0.6, \mathrm{Re}_{n}=6.3 * 10^{7}$

6.40 shows that for the full-scale propeller the extent of the leading edge cavity is larger while the tip vortex cavity is smaller. This result is similar to the previous results concerning the leading edge cavity. While for the tip vortex, the distribution for the full scale geometry is similar to the one for the model scale geometry, in wake the cavitating vortex is smaller. 


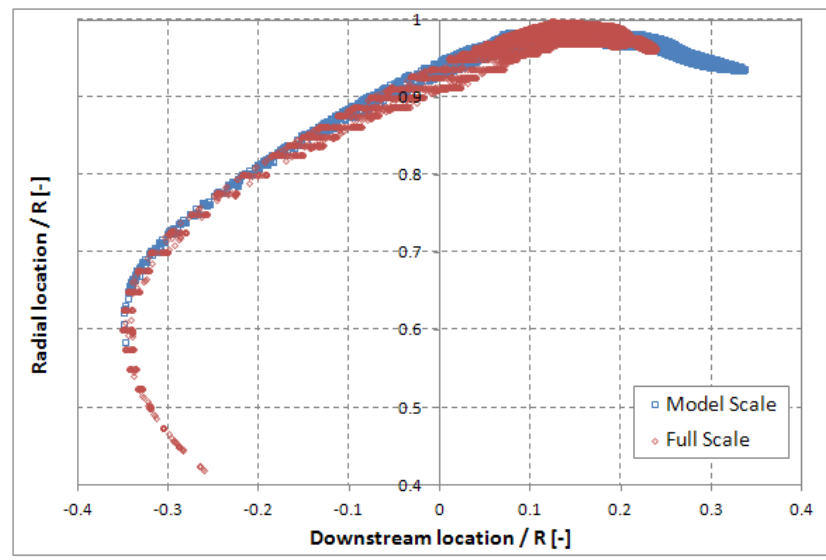

Figure 6.60: Leading Edge Skew Propeller in cavitating flow $(\sigma=2.27)$. Radial location of minimum pressure coefficient and iso-surface $C_{p}=-2.27$ as function of downstream location. $\mathrm{J}=0.6, \mathrm{Re}_{n}=6.3 * 10^{7}$. Comparison of full-scale results and model-scale results

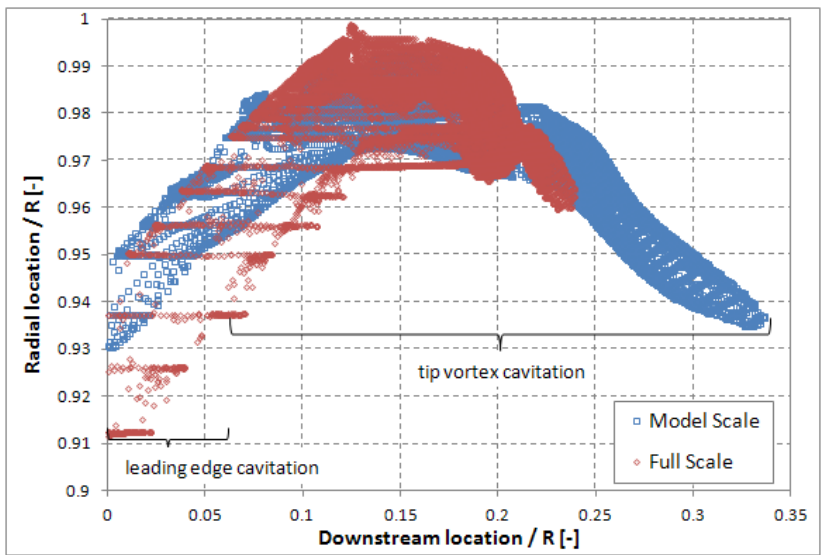

Figure 6.61: Leading Edge Skew Propeller in cavitating flow $(\sigma=2.27)$. Radial location of iso-surface $C_{p}=-2.27$ as function of downstream location. $\mathrm{J}=0.6, \mathrm{Re}_{n}=6.3 * 10^{7}$. Comparison full-scale results and model-scale results

The radial location of the tip vortex position determined from the iso-surface $C_{p}=-2.27$ for model scale and full scale is presented in figure 6.60. It is 
again visible that the extent of the leading edge vortex at full scale is smaller then at model scale. Also the fast decay of the tip vortex due to the insufficient grid resolution is clear. Zooming into the region of the tip vortex, see figure 6.61 , the cross-sectional size of the leading edge cavitation is decreasing towards the region of the tip vortex core. This is similar for both full scale and model scale close to the propeller tip but different in downstream direction, i.e. there the cavity is smaller in length for the vortex at the fullscale when compared with the vortex at model scale. The reason for this fast decay of the full-scale tip vortex appears to be caused by the grid being not fine enough for the full-scale Reynolds number.

All the results on the scaling of the tip vortex are numerical and in the following an overview is given of the experimental scaling as extracted from ITTC reports.

The Reynolds scaling effect on the azimuthal velocity in the tip vortex, can be assessed from the 21st ITTC Cavitation Committee [2] report. This report states that:

"It has been observed that the tip vortex core size is reduced as the Reynolds number is increased. Physically, it seems reasonable that if the core size becomes smaller, the vortex will be more 'tightly wound' and we could expect the magnitude of the peak tangential velocity would be larger."

This can be translated into: The full scale vortex core will be smaller in dimensionless cross-sectional size. Correspondingly the velocity and pressure have a larger gradient. This is not in accordance with the present findings, in which the full-scale tip vortex has slightly smaller dimensionless gradients than found for model scale. This indicates that the grid requirements for the full scale vortex are not satisfied to the full extent.

However, according to Kuiper [43]:

"The radius of the cavitating core of the tip vortex is independent of Reynold number and nuclei content."

This last remark agrees with the present findings, though in the wake the full scale tip vortex decays too fast in the results of the numerical simulations, primarily due to grid resolution but possibly also due to the too dissipative turbulence modeling. 


\subsubsection{Conclusions}

The numerical simulations for the Leading Edge Skew Propeller have been assessed for wetted and cavitating flow conditions for both the model and full-scale propeller.

The numerical simulations for the model-scale propeller do predict tip vortex cavitation inception in close agreement with the results of the model tests when using the minimum pressure coefficient computed for wetted flow as indication for inception of cavitation. The cavitating flow reveals the presence of a clear leading-edge tip vortex as observed in the experiments, while in the wake the cavitating tip vortex diffuses relatively fast in downstream direction, as observed for all the cases analysed. The latter is mainly attributed to insufficient grid resolution and too dissipative turbulence modeling, see [89] and [34].

Numerical simulations for the full-scale propeller do predict the expected performance scaling with clear effects of the higher Reynolds number on the minimum pressure coefficient. The distribution of the pressure and velocity inside the tip vortex core are similar to the ones for the model-scale propeller. This is in agreement with Kuiper's (see [43]) observations.

The Reynolds number scaling effects within the tip vortex core as observed in the results of the numerical simulations can be attributed to the grid which is required to be finer for the full-scale propeller in comparison to the modelscale propeller. This agrees with the 21st ITTC Cavitation Committee [2] remarks on tip vortex cavitation scaling.

As a recommendation for future numerical simulations for the full scale propeller, the resolution of the grid should be increased compared to the mesh for the model-scale propeller, i.e. keeping the $y^{+}$within the required bounds (as in the present numerical simulations for the Leading Edge Skew propeller). Still, the simulations for the full-scale propeller do also predict the expected tip vortex cavitation scaling and a clear leading edge - tip vortex cavitation even though in the wake the tip vortex is very fast decaying due to insufficient mesh resolution and too dissipative turbulence modeling.

\subsection{Conclusions}

The numerical simulations for the open propeller with tip vortex cavitation, at model scale (propeller 4119DTRC and Leading Edge Skew propeller) and full scale (Leading Edge Skew propeller) have been investigated. The main goal has been the assessment of the capabilities of the numerical simulations 
to predict tip vortex cavitation inception and the development of the cavitating tip vortex. These results are of utmost importance since this type of cavitation is frequently present on marine propellers.

The results for the wetted flow show that numerical simulations are capable to predict propeller performance within $6 \%$ of the data from the measurements. Deviations are mainly related to the occurrence of a different flow regime. Numerical simulations have been carried out for fully turbulent flow, while in measurements both laminar and turbulent flow is present.

Results for tip vortex cavitation inception for the two propeller test cases analysed are well in agreement with the cavitation curves obtained from experiment, see figures 6.17 and 6.38. Also note that, inception curves for model-scale propeller are strongly influenced by:

- the nuclei contents of the water, see [2] and [43]

- the roughness of the propeller blades (mainly applied at leading edge) and the Reynolds number at which measurements are performed, see [2], [52] and [43]

- the method of observation of cavitation: visual or acoustical

- the person that performs the observation inception (if visual, different persons will observe the inception at different a cavitation number).

Thus the agreement with the experimental inception curves found in figures 6.17 and 6.38 can be considered adequate to be used in propeller design stage.

From the results, it is also clear that the pressure coefficient, the velocity field and the Q-factor give a good indication of the type of cavity and of the location / presence of the tip vortex already for non-cavitating flow for which the required computational resources are much less demanding than for cavitating flow.

The main results of the numerical simulation of cavitating flow do predict tip vortex cavitation for the 4119 DTRC propeller (at model scale) and leading edge-tip vortex cavitation for the Leading Edge Skew propeller (at model scale and full scale). The issue of the numerical results for both model scale and full scale is the extent of the predicted tip vortex cavitation. Although the mesh is refined in the region where the tip vortex forms, utilising the developed method based on low values of the pressure and high values of the Q-factor (see Vortex Detection and Visualisation chapter), this appears not to be sufficient to capture the tip vortex in the region further downstream. The reason for this fast decay of the predicted tip vortex is due to insufficient 
grid resolution as well as due to diffusion and dissipation of the tip vortex induced by the turbulence model. The solution for these issues might be a better automated refinement of the grid in the tip vortex and the use of a more advanced mathematical model like the RSM, LES/DES or DNS, see [51] and [34]. Another popular approach in the last years, that could be practical for industry, is the zonal approach in which RANS is still used at the wall and LES/DES are used in the rest of the field, as in the Flomania EU project, see [58] and [57]. Note also, that the numerical simulations for full scale propellers require finer grid in comparison to the model scale propellers.

Concluding, based on the current study, numerical simulations employing RANS can be used effectively for the prediction of:

- pressure and velocity fields, for wetted and cavitating flows at model scale or full scale

- lift and drag or thrust and torque predictions, for wetted and cavitating flow at model scale as well as at full scale

- tip vortex cavitation inception, assessed using results for the wetted flow at model scale as well as at full scale

- tip vortex and leading edge-tip vortex cavitation and its downstream development close to the blade tip, for cavitating flow at model scale as well as at full scale

- possible indication of erosion, based on the shed clouds and surface pressure distribution for cavitating flow at model scale as well as at full scale. This is not fully investigated in the present thesis, for further details see also findings in [47].

Numerical simulations based on the RANS equations have limited use for:

- vortex-rudder/ship interaction because in the numerical simulations the cavitating tip vortex is fast decaying in downstream direction, especially for the full scale propeller. For such predictions, more advanced mathematical models have to be considered. These models have not been considered in the present thesis because of their requirements in terms of computational resources which are not yet practical within industry.

Compared to the capabilities of a model-test facility, RANS simulations are at least equivalent (predictable are: performance, cavitation inception and 
tip vortex presence) while giving much more insight into the flow field. The use of the simulations in the design stage is recommended in the beginning of the design process and in conjunction with the experimental approach by the end of the design stage if required. 


\section{Chapter 7}

\section{Conclusions and Recommendations}

In this last chapter conclusions and recommendations for future work are given.

The present $\mathrm{PhD}$ study has as purpose identifying and filling the gaps in the methods for numerical simulations of propeller cavitating flows.

The numerical method will be used in the daily design process for predicting cavitating flows, especially the tip vortex cavitation inception and development on full-scale hydrofoils and propellers. Taking into account the requirements on the accuracy of the method and its use of computer resources, the RANS approach has been selected. The main conclusions on the selected approach, its features and capabilities, are summarized in the present chapter. Finally, recommendations for further study are outlined.

\subsection{Numerical method}

Numerical simulations of cavitating flow intended to be used in industry require a relatively fast numerical method (answers on design questions within a week) while keeping the fidelity of the mathematical model of the flow also high (at least a viscous flow method required). An analysis of the current state-of-the-art numerical methods, given in the first chapter (Introduction), indicates that the RANS approach is the most suitable approach when compromising between the requirements of physical fidelity and those of computational resources required. The applied numerical method resulting from the study performed in chapters 3 and 4 , is used for numerical simulations of cavitating flow in this thesis (chapters 5 and 6 ). This numerical method 
is summarized in table 7.1.

\begin{tabular}{|c|c|}
\hline Aspect & Approach \\
\hline Governing equations & RANS mathematical model \\
\hline Turbulence modeling & modified RNG $k-\varepsilon$ turbulence model \\
\hline Cavitation modeling & Sauer cavitation model \\
\hline Differencing scheme, space & MARS scheme (2nd order) \\
\hline Differencing scheme, time & Euler scheme (1st order) \\
\hline Vortex detection & Cp and Q-factor \\
\hline Average surface grid & $1 \%$ from a representative length \\
\hline Vortex grid & refinement based on distribution of Cp and Q-factor \\
\hline Time step & 20 \\
\hline Inner iterations & \\
\hline
\end{tabular}

Table 7.1: Summary of the method used for cavitating flows

Using the above table and the appropriate boundary conditions, for wetted and cavitating flow, the results found in chapters 5 and 6 have been obtained. The main conclusions of the thesis on the results of wetted and cavitating flow are presented in the following.

\subsection{Conclusions on results for wetted flow}

For the wetted flow the results for the Twist 11 hydrofoil (Chapter 5), show that lift and pressure are predicted within about $5 \%$ of the measurements. The method predicts hydrofoil lift and pressure distribution within the desired accuracy (at least $5 \%$ required).

In chapter 6, the results for the 4119DTRC Propeller, show that the method can predict the propeller performance, the inception of the tip vortex and the wake development as well as the location of the tip vortex (close to the blade, in terms of distribution of azimuthal velocity and pressure as well as Q-factor) as observed in the experimental observation by Jessup in [39].

The results for the Leading Edge Skew Propeller show that the method can predict the performance as well as the leading edge-tip vortex inception and tip vortex development (close to the blade) as observed in experiments, see [35].

The results for the full-scale Leading Edge Skew Propeller show that the method predicts the performance scaling (mainly on thrust and efficiency) similar to the scaling in the ITTC guidelines, see [3]. The scaling of the 
leading-edge tip vortex inception is predicted in the simulations but for lower power than expected from the model test. The method also predicts the propeller leading edge-tip vortex development and wake, which decays in downstream direction faster than the tip vortex in the results for the model-scale propeller. This is mainly due to insufficient grid resolution for this higher Reynolds number and due to dissipative effects caused by the turbulence model employed.

\subsection{Conclusions on results for cavitating flow}

The method gives the expected prediction in terms of the shedding frequency and the character of the cavity macro structure, in line with the model test observations for the Twist 11 hydrofoil. The results are discussed in chapter 5 for which the experimental observations can be found in [29]. Note that the cavity shedding is obtained utilising the modified eddy viscosity and the RNG $k-\varepsilon$ turbulence model as detailed in chapter 2. However, the small vortex structures, observed in the cavitation tests, are not captured due to insufficient grid resolution (finer grids are needed to compute the small size eddies) and turbulence modeling (two-equations turbulence models cause vortical structures to dissipative over a short distance). Finer grids and more advanced turbulence models should be considered if the small structures / vortices are to be captured.

The results for the Elliptic 11 Rake hydrofoil in cavitating flow do show a clear leading edge-tip vortex cavitation, similar to the observations in experiment [87]. However, in the wake the cavitating tip vortex decays fast due to insufficient grid resolution and dissipative turbulence modeling.

The method predicts the cavitation inception curves of the 4119 DTRC propeller in good agreement with the experimental curves. Also, a clear tip vortex is present in the results of the simulations but again fast decaying downstream of the propeller.

The results of the numerical simulations for the Leading Edge Skew Propeller demonstrate the capability of the method to predict tip vortex inception. Furthermore, the leading edge-tip vortex cavitation correlates quite well with experimental observation. The shortcoming of the method is the extent of the tip vortex, which has to be improved with a higher mesh resolution. This has been demonstrated for one blade, though further refinement could not be achieved due to the limit on the number of cells. The effects of more advanced turbulence modeling follow from the study of the NACA0015 hydrofoil and Twist 11 hydrofoil, for which the grid refinement showed no 
improvement in cavitation prediction and an advanced turbulence model did influence the results.

The obtained results for the full propeller indeed provide the Reynolds scaling for tip vortex cavitation inception. However, the power in the McCormick's expression for the Reynolds scaling is slightly lower than found from model tests. In the wake, the full scale tip vortex is decaying even faster than on model scale since the grid resolution in this region should be higher at full scale. The main limitation of the method is that the meshes for full-scale propellers should be very fine. Also a more advanced turbulence model is expected to improve the tip vortex in the wake.

\subsection{Recommendations for future study}

The main issues not resolved in the current study are the development of the tip vortex downstream of the configuration, especially at full scale. Furthermore, the unsteadiness of the tip vortex. Although, the last issue was not within the scope of the thesis it is of utmost importance when analysing tip vortices.

Further investigations are needed to capture accurately the development in the downstream direction of the tip vortex. This concerns the grid refinement as well as turbulence and cavitation models. This is required in order to address the unsteadiness of the tip vortex cavitation, which leads to radiated pressure fluctuations, erosion and rudder/ship interaction.

Part of the limitation in representing the full development of the tip vortex is attributed to the grid. However, more advanced turbulence models, like LES or DES also require fine grids in order to capture the smallest eddy structures.

Once the tip vortex is satisfactorily captured in numerical simulations, the assessment of the pressure fluctuations, the erosion and the radiated noise can be investigated for rudder/ship-propeller configurations.

In this thesis, only the Sauer cavitation model (as implemented in StarCD) has been investigated. For future studies alternative models should be analysed (see also [31]) and assessed for numerical simulation of tip vortex cavitation. 


\section{Bibliography}

[1] 20th ITTC International Towing Tank Conference. Propulsor Committee. San Francisco, California, 1993.

[2] 21st ITTC Proceedings. Cavitation Committee, Final Report and Recommendations to the 21st ITTC. Trondheim, 1996.

[3] 22nd ITTC International Towing Tank Conference. Propulsor Committee. Seoul and Shanghai, 1999.

[4] ITTC 22nd; Recommended Procedures and Guidelines. ITTC - Quality Manual, CFD General, Uncertainty Analysis in CFD, Uncertainty Assessment Methodology. 1999.

[5] I.H. Abbot and A.E. von Doenhoff. Theory of wing sections. Dover Publications, inc., 1959.

[6] M. Abdel-Maksoud. Numerical and Experimental Study of Cavitation Behaviour of a Propeller. 2003.

[7] R.E.A. Arndt, C.C.S. Song, M. Kjeldsen, J. He, and A. Keller. Instability of partial cavitation: A numerical/experimental approach. 23rd Symposium on Naval Hydrodynamics, France, 2000.

[8] D.C. Banks and B.A. Singer. Vortex Tubes in Turbulent Flows: Identification, representation, reconstruction. IEEE Visualization94, 1994.

[9] G.K. Batchelor. An Introduction to Fluid Dynamics. Cambridge University Press, 2000.

[10] C.E. Brennen. Hydrodynamics of Pumps. Oxford University Press, 1994.

[11] C.E. Brennen. Cavitation and Bubble Dynamics. Oxford University Press, 1995. 
[12] C.E. Brennen. Fundamentals of Multiphase Flows. Cambridge University Press, 2005.

[13] N. Bulten and A.I. Oprea. Consideration on deviations in torque prediction for propellers and waterjets with RANS codes. RINA, 2005.

[14] N. Bulten and A.I. Oprea. Evaluation of McCormick's rule for propeller tip cavitation inception based on CFD results. CAV2006, Wageningen, The Netherlands, 2006.

[15] J. Carlton. Marine propellers and propulsion. Elsevier Ltd., 2007.

[16] CD-adapco. STAR-CD Version 4.02 CCM User Guide. CD-adapco Group, 2006.

[17] CD-adapco. STAR-CD Version 4.02 Methodology. CD-adapco Group, 2006.

[18] T. Cebeci, J.P. Shao, F. Kafyeke, and E. Laurendeau. Computational Fluid Dynamics for Engineers. Springer, 2005.

[19] T. Cebeci and A.M.O. Smith. Analysis of Turbulent Boundary Layers. Academic Press, 1974.

[20] W.-P. Cheng and B. Cantwell. A study of the fine scale motions of the incompressible time-developing mixing layers. Center for Turbulence Research, Proceeding of the Summer Program, 1992.

[21] W.-P. Cheng and B. Cantwell. Study of the velocity gradient tensor in turbulent flow. Department of Aeronautics and Astronautics, Stanford University, 1996.

[22] C. Chesnakas and S. Jessup. Experimental Characterization of Propeller Tip Flow. 22nd Symposium on Naval Hydrodynamics, 1998.

[23] J-K Choi, G.L. Chahine, and C-T Haiso. Characteristics of bubble splitting in a Tip Vortex. CAV2003, 2003.

[24] O. Coutier-Delagosha, J.L. Reboud, and B. Stutz. Two phase flow structure of cavitation: experiment and modeling of unsteady effects. Third International Symposium on Cavitation, 1998.

[25] S. Danaila. Courses on Computational Fluid Dynamics. Aerospace Faculty, University of Politechnica Bucharest, 1997-2003. 
[26] Davidhv22. http://en.wikipedia.org/wiki/File:Cavitacion.jpg. Wikipedia, 2011.

[27] M. Dular, R. Bachert, and B. Stoffel. Experimental and numerical investigation of swept leading edge influence on the developed cavitation. Sixth International Symposium on Cavitation, CAV2006, Wageningen, The Netherlands, 2006.

[28] J.H. Ferziger and M. Peric. Computational Methods for Fluid Dynamics. 3rd Edition, Spriner Book, 2002.

[29] E-J Foeth. The structure of three-dimensional sheet cavitation, PhD Thesis. Delft University of Technology, 2008.

[30] J-P Franc and J-M Michel. Fundamentals of Cavitation. Springer, 2004.

[31] S. Frikha, O. Coutier-Delgosha, and J. A. Astolfi. Influence of the Cavitation Model on the Simulation of Cloud Cavitation on 2D Foil Section. International Journal of Rotating Machinery, Hindawi Publishing Corporation, Volume 2008, Article ID 146234.

[32] R. Ghias, R. Mittal, H. Dong, and T.S. Lund. Study of Tip-Vortex Formation Using Large-Eddy Simulation. 43rd Fluid Dynamics Conference and Exhibit, AIAA, 2005.

[33] C. Hirsch. Numerical computation of internal and external flows. Wiley, New York, 1991.

[34] C.-T. Hsiao and G. L. Chahine. Scaling of Tip Vortex Cavitation Inception for a Marine Open Propeller. 27th Symposium on Naval Hydrodynamics, Seoul, Korea, 5-10 October 2008.

[35] Leading Edge Technical Report HSVA. Prediction of Leading Edge and Tip Flow for the Design of Quiet and efficient Screw Propellers. 2002.

[36] T. Huuva. Large eddy simulation of cavitating and non-cavitating flow, PhD Thesis. Chalmers University of Technology, Göteborg, Sweden, 2008.

[37] Virtual Towing Tank Utility in Europe. European Commission Project, FP6. http://www.virtual-basin.org/, 2005-2008.

[38] J. Jeong and F. Hussain. On the Identification of a Vortex. Journal of Fluid Mechanics, 1995. 
[39] S.D. Jessup. An experimental investigation of viscous aspects of propeller blade flow, PhD Thesis. 1989.

[40] M. Jiang, R. Machiarju, and D. Thompson. Detection and Visualization of Vortices. Visualization Handbook, 2005.

[41] S. J. Kline, S. K. Robinson, and P. R. Spalart. A review of quasi-coherent structures in a numerically simulated turbulent boundary layer. NASA TM 102191, 1989.

[42] A.H. Koop. Numerical Simulation of Unsteady Three-Dimensional Sheet Cavitation, PhD Thesis. University of Twente, 2008.

[43] G. Kuiper. Cavitation inception on propellers, PhD Thesis. 1990.

[44] R.F. Kunz, D.R. Stineberg, D.A. Boger, T.S. Chyczewski, J.W. Lindau, and T. R. Govindan. Multi-phase CFD analysis of natural and ventilated cavitation about submerged bodies. Proceedings of the 3rd ASME/JSME Joint Fluids Engineering Conference, 1999.

[45] R.S. Laramee, H. Hauser, H. Doleisch, B. Vrolijk, F. H. Post, and D. Weiskopf. The State of the Art in Flow Visualization: Dense and Texture-Based Techniques. Computer Graphics Forum, Vol. 23, No. 2, 2004, pages 203-221, 2004.

[46] W. Lauterborn. Cavitation and Inhomogeneities in Underwater Acoustics. Spronger-Verlag, 1980.

[47] Q. Le, J.P. Franc, and J.M. Michel. Partial cavities: Global behavior and mean pressure distribution. Journal of Fluids Engineering, 115:243-248, 1993.

[48] Y. Levy, D. Degani, and A. Seginer. Graphical Visualization of Vortical Flows by Means of Helicity. AIAA 28(8), pp. 1347/1352, 1990.

[49] D-Q Li, N. Berchiche, and C-E Janson. Influence of Turbulence Models on Prediction of Full-Scale Propeller Open Water Characteristics with RANS methods. 26th Symposium on Naval Hydrodynamics, 2006.

[50] Z. Li. Assessment of Cavitation Erosion with a Multiphase ReynoldsAveraged Navier-Stokes Method, PhD thesis. TUDelft, 2012.

[51] C. Lifante, T. Frank, and K. Rieck. Investigation of pressure fluctuations caused by turbulent and cavitating flow around a P1356 ship propeller. RINA Marine CFD, 2008. 
[52] J.T. Ligtelijn, J. Kooij, G. Kuiper, and W. van Gent. Research on Propeller-Hull Interaction in the Depressurized Towing Tank. 1992.

[53] H.J. Lugt. Vortex Flow in Nature and Technology. Wiley, 1972.

[54] B.W. McCormick. On Cavitation Prediction by a Vortex Trailing from a Lifting Surface. 1962.

[55] F. R. Menter. Zonal Two Equation k- $\omega$ Turbulence Models for Aerodynamic Flows. AIAA Paper 93-2906, 1993.

[56] F. R. Menter. Two-Equation Eddy-Viscosity Turbulence Models for Engineering Applications. AIAA Journal, vol. 32, no 8. pp. 1598-1605., 1994.

[57] F. R. Menter and M. Kuntz. Adaptation of Eddy-Viscosity Turbulence Models to Unsteady Separated Flow Behind Vehicles. The Aerodynamics of Heavy Vehicles: Trucks, Buses and Trains, Asilomar, Ca, Springer, 2004.

[58] F. R. Menter, M. Kuntz, and R. Langtry. Ten Years of Industrial Experience with the SST Turbulence Model. Turbulence, Heat and Mass Transfer 4, 2003.

[59] P. Moin and J. Kim. The structure of vorticity field in turbulent channel flow. Part 1. Analysis of instantaneous fields and statistical correlations. Journal of Fluid Mechanics 155, 441-464, 1985.

[60] P. Moin and J. Kim. The structure of vorticity field in turbulent channel flow. Part 2. Study of ensemble-averaged fields. Journal of Fluid Mechanics 162, 339-363, 1986.

[61] J.G. Moore and J. Moore. Controlling over-production of turbulence in two-equation models by limiting the anisotropy of the Reynolds normal stresses. ASME Fluids Engineering Division Summer Meeting, 1997.

[62] Sir Isaac Newton. Opticks: or a Treatise of the Reflections, Refractions, Inflections and Colours of Light. 1704.

[63] C.A. Parsons. The steam turbine on land and at sea. 1906.

[64] P. Pennings. Private comunication. TU Delft, 2013.

[65] S.B. Pope. Turbulent Flows. Cambridge University Press, 2000. 
[66] LEADING EDGE EU Project. Prediction of Leading Edge and Tip Flow for the Design of Quiet and Efficient Screw Propellers. Contract No:G3RD-CT-2002-00818, 2002.

[67] O. Reynolds. Transactions of the Institution of Naval Architects. 1874.

[68] O. Reynolds. Papers on Mechanical and Physical Subjects. Cambridge at the University Press, 1900.

[69] S.H. Rhee and S. Joshi. Computational Validation for Flow around a Marine Propeller Using unstructured Mesh Based Navier-Stokes Solver. JSME International Journal, 2005.

[70] P.J. Roache. Verification and Validation in Computational Science and Engineering. 1998.

[71] S. K. Robinson. Coherent motions in the turbulent boundary layer. 1991.

[72] J. Sauer. Instationär kaviterende Strömung - Ein neues Modell, basierend auf Front Capturing (VoF) und Blasendynamik, PhD Thesis. Fakultät fur Maschinenbau Karlsruhe, 2000.

[73] H. Schlichting and K. Gersten. Boundary Layer Theory. 8th Revised and Enlarged Edition, Spinger Book, 2000.

[74] S.J. Schmidt, I.H. Sezal, G.H. Schnerr, and M. Thalhamer. Shock Waves as Driving Mechanism for Cavitation Erosion. Proceedings of the 8th International Symposium on Experimental and Computational Aerothermodynamics of Internal Flows, 2007.

[75] G.H. Schnerr and J. Sauer. Physical and numerical modeling of unsteady cavitation dynamics. Proceedings of the 4th International Conference on Multiphase Flow, 2001.

[76] Y. Shen, G. Chahine, C-T Hsiao, and S. Jessup. Effects of model size and free stream nuclei on tip vortex cavitation inception scaling. CAV2001, 2001.

[77] K. W. Shin, P. Andersen, and R. Mikkelsen. Cavitation Simulation on Conventional and Highly-Skewed Propellers in the Behind-Hull Condition. Second International Symposium on Marine Propulsors, SMP'11, Hamburg, Germany, 2011. 
[78] A.K. Singhal, M.M. Athavale, H. Li, and Y. Jiang. Mathematical basis and validation of the full cavitation model. Journal of Fluids Engineering, vol. 124, no. 3, pp. 617-624, 2002.

[79] S. Stegmaier, U. Rist, and T. Ertl. Opening the Can of Worms: An Exploration Tool for Vortical Flows. In Proceedings of IEEE Visualization, 2005.

[80] F. Stern, R. Wilson, and J. Shao. Quantitative Verification and Validation of CFD simulations and certification of CFD codes. 2005.

[81] D.R. Stinebring, R.F. Kunz, and D.A. Boger. A preconditioned NavierStokes method for two-phase flows with application to cavitation prediction. Computers \& Fluids, vol.29, no.8, pp.849-875, 2000.

[82] H. Tennekes and J.L. Lumley. A First Course in Turbulence. The MIT Press, 1972.

[83] T.J.C. Van Terwisga, P. A. Fitzsimmons, Z. Li, and E.J. Foeth. Cavitation Erosion: A review of physical mechanisms and erosion risk models. Proceedings of the 7th International Symposium on Cavitation, CAV2009, Ann Arbor, Michigan, USA, 2009.

[84] J. Thornycroft and S.W. Barnay. Torpedo-boat destroyers. Minutes of the Proced. Inst. Civil Engrs., 1895.

[85] A. Tsinober. Turbulent Flows. Springer, 2004.

[86] A. Vabre, O. Coutier-Delagosha, B. Stutz, and S. Legoupil. Analysis of cavitating flow structure by experimental and numerical investigations. Journal of Fluid Mechanics, 578:171-222, 2007.

[87] A.J. van der Hout. The interaction of sheet and vortex cavitation, MSc thesis. Delft University of Technology, 2007.

[88] J. Villasenor and A. Vincent. An algorithm for space recognition and time tracking of vorticity tubes in turbulence. CVGIP: Image Understanding, 55, 27-35, 1992.

[89] J. Wells. Effects of Turbulence Modeling on RANS Simulations of Tip Vortices, MSc thesis. Virginia Polytechnic Institute and State University, 2009.

[90] P. Wesseling. Principles of computational fluid dynamics. Springer, 2001 
[91] D.C. Wilcox. Turbulence modeling for CFD. DCW Industries, Incorporated; 3rd Edition, 2006.

[92] A. A. Wray, J. C. R. Hunt, and P. Moin. Eddies, stream, and convergence zones in turbulent flows. Center for Turbulence Research Report CTRS88, 1988.

[93] V. Yakhot and S.A. Orszag. Renormalization group analysis of turbulence-I: Basic Theory. J. Scientific Computing, 1, pp. 1-51, 1986.

[94] V. Yakhot and S.A. Orszag. Development of turbulence models for shear flows by a double expansion technique. Phys. Fluids, A4(7), pp. 15101520, 1992.

[95] L. A. Yates and G. T. Chapman. Streamlines, Vorticity Lines and Vortices. AIAA 91-0731, 1991.

[96] N.J. Zabusky, O.N. Boratav, R.B. Pelz, M. Gao, D. Silver, and S.P. Cooper. Emergence of coherent patterns of vortex stretching during reconnection: A scattering paradigm. Phys Rev Lett. 1991 Oct 28;67(18):2469-2472, 1991. 


\section{Solution of cubic equation}

Consider the cubic equation:

$$
\lambda^{3}+a_{1} \lambda^{2}+a_{2} \lambda+a_{3}=0
$$

where all coefficients are real-valued. This equation can be re-written as:

$$
\left(\lambda+\frac{1}{3} a_{1}\right)^{3}+\left(a_{2}-\frac{3}{9} a_{1}\right)\left(\lambda+\frac{1}{3} a_{1}\right)+\left(\frac{2}{27} a_{1}-\frac{1}{3} a_{1} a_{2}+a_{3}\right)=0
$$

This leads to:

$$
\eta^{3}+3 Q \eta-2 R=0
$$

with

$$
\begin{gathered}
\eta=\lambda+\frac{1}{3} a_{1} \\
3 Q=a_{2}-\frac{1}{3} a_{1}^{2} \\
2 R=-\left(\frac{2}{27} a_{1}^{3}-\frac{1}{3} a_{1} a_{2}+a_{3}\right)
\end{gathered}
$$

Using

$$
S=\left[R+\sqrt{R^{2}+Q^{3}}\right]^{1 / 3}
$$

and

$$
T=\left[R-\sqrt{Q^{3}+R^{2}}\right]^{1 / 3}
$$

the solution follows with the discriminant $\Delta=Q^{3}+R^{2}>0$ as

$$
\begin{gathered}
\lambda_{1}+\frac{1}{3} a_{1}=S+T \\
\lambda_{2}+\frac{1}{3} a_{1}=-e^{-i \pi / 3} S-e^{i \pi / 3} T \\
\lambda_{3}+\frac{1}{3} a_{1}=-e^{i \pi / 3} S-e^{-i \pi / 3} T
\end{gathered}
$$


In equation above, both $S$ and $T$ are real-valued as long as the discriminant $\Delta$ is positive.

In case the discriminant $\Delta=Q^{3}+R^{2}<0$, all three eigenvalues will be real-valued:

$$
\lambda_{1}+\frac{1}{3} a_{1}=2 \sqrt{-Q} \cos \left(\frac{1}{3} \theta\right)
$$

with $\cos \theta=R / \sqrt{-Q^{3}}$

$$
\begin{aligned}
& \lambda_{2}+\frac{1}{3} a_{1}=2 \sqrt{-Q} \cos \left(\frac{1}{3}(\theta+2 \pi)\right) \\
& \lambda_{3}+\frac{1}{3} a_{1}==2 \sqrt{-Q} \cos \left(\frac{1}{3}(\theta+4 \pi)\right)
\end{aligned}
$$

Finally, since $\lambda^{3}+a_{1} \lambda^{2}+a_{2} \lambda+a_{3}=\left(\lambda-\lambda_{1}\right)\left(\lambda-\lambda_{2}\right)\left(\lambda-\lambda_{3}\right)=\lambda^{3}-\left(\lambda_{1}+\lambda_{2}+\right.$ $\left.\lambda_{3}\right) \lambda^{2}+\left(\lambda_{1} \lambda_{2}+\lambda_{1} \lambda_{3}+\lambda_{2} \lambda_{3}\right) \lambda-\lambda_{1} \lambda_{2} \lambda_{3}=0$, the roots of the cubic equation satisfy the following identities:

$$
\begin{array}{r}
\lambda_{1}+\lambda_{2}+\lambda_{3}=-a_{1} \\
\lambda_{1} \lambda_{2}+\lambda_{1} \lambda_{3}+\lambda_{2} \lambda_{3}=a_{2} \\
\lambda_{1} \lambda_{2} \lambda_{3}=-a_{3}
\end{array}
$$




\section{Acknowledgements}

This thesis would have not been possible without the help, support and encouragement of many people along the years.

I particularly have to thank Wärtsilä to offer me the opportunity of carrying out my research and supporting it financially, materially and professionally. The first person that gave me the confidence and offered me the opportunity to start the $\mathrm{PhD}$ while working at Wärtsilä, for which I will always be thankful, is Do Ligtelijn. Your encouragement and support made the start of this thesis possible.

All of my thanks to Prof.Harry Hoeijmakers and Prof.Tom van Terwisga for being along with me on this journey and helping me in achieving its goal and seeing the light at the end of the tunnel. Your work and support made the completion of this thesis possible.

I would like to specially thank Norbert Bulten for creating the CFD department inside Wärtsilä Netherlands and for all his support and theoretical and practical work, which is the basis of this thesis. Thanks towards all of my colleagues from the CFD team along the years: Brigitte Fumex, Petra Stoltenkamp, Igor Denissen, Maarten Bijlard, Koen in de Braekt, Edwin Munts and Mark Bijlsma. You all supported me practically, in giving me lots of valuable ideas, and mentally since most of you have been on this route towards a $\mathrm{PhD}$.

I would like to thank all the MSc and $\mathrm{PhD}$ students met along the years in various meetings and fruitful discussions, but especially: Li Ziru, Evert-Jan Foeth, Arjen Koop and Pepijn Pennings. Also Martin Hoekstra, Guilherme Vaz and Johan Bosschers, from MARIN, met often within project meetings like Leading Edge and VIRTUE or different PhD meetings. The majority of the test cases within the thesis were gathered from these EU-projects.

My friends: Cami, Alina, Ellen, Katerina, Cristina, Stefan, Basti, Aka and Silvester, 'Thanks guys!' you supported and encouraged me through the years when I was not the most relaxed person to have around on many occasions.

All my gratitude to my parents, all of my achievements and success is only 
due to their way of bringing me up. My brother is one of my closest friends and all our discussions along these years helped a lot.

Last salute is towards my partner in everything over the last decade, my 'Wall-E'. Always being there for me, makes everything possible. 


\section{Curriculum Vitae}

January 1979 Born in Bucharest, Romania

1993-1997 National College Iulia Hasdeu, Bucharest

1997-2002 The Faculty of Aerospace Engineering, University Politehnica of Bucharest, final thesis in CFD "Numerical Modelling of Navier-Stokes Equations for Incompressible Flows Applied to Internal Flows"

2002-2003 Research Scientist, Subsonic Wind Tunnel, National Institute for Aerospace Research "Elie Carafoli", Bucharest

2002-2003 Advanced studies (MSc), dissertation project in CFD "Numerical Schemes Study for Burgers Equation", The Faculty of Aerospace Engineering, University Politehnica of Bucharest

2004 PhD course, J.M. Burgers Center, "Finite Element Methods for the Incompressible Navier-Stokes Equations and Iterative Solution Methods", TU Delft

2003-2005 Marie-Curie Fellowship "Optimising vessel safety and propulsive performance by optimising computational design", Wärtsilä

2005-present Specialist Numerical Analysis, CFD department, Propulsion R\&D, Wärtsil̈̈ 
